\title{
Measuring the Disappearance of Muon Neutrinos with the MINOS Detectors
}

A thesis submitted to University College London for the degree of

Doctor of Philosophy

in the Faculty of Physical Sciences

August 2013

Alexander Radovic

Department of Physics and Astronomy 
The last few years have been a long and exciting adventure, and I am in the debt of a great many friends, colleagues and family members. I have had a great many mentors, not least of all my supervisor Prof. Jenny Thomas who has been a constant source of sage wisdom, often when I didn't even know I needed it. Thank you for you honest advice and support over these past 3 years.

I still remember my first fledgling months working with MINOS within the "disappearance group", and I would like to thank Justin Evans for his support, guidance, and patience as I learned the ropes of running an analysis. Thanks also to Ryan Nichol who has been putting up with me since I first worked on a summer project at UCL with Anita in 2009. Thanks to Robert Hatcher and Art Kreymer, I suspect I have learnt more from overheard snippets of conversation between the two of you than I could find in the best of computing textbooks. Thanks to the many great scientists at Fermilab, UCL, and within MINOS who have shared their wisdom with me along the way.

I have been blessed with a great many friends scattered across the globe and should like to thank them all for making the last few years the rare pleasure that it has been. Jamie, Liam and Chris. You remain the closest thing to brothers that I have, and I wish you all the very best in your own adventures. Particular thanks go to my very dear friends Cindy Joe and Joseph Zennamo who braved my terrible spelling and grammar to help edit the thesis you are reading now.

Finally, none of this would have been possible without the loving support of my family. Mum, Gran, Brana, and Anna- thank you so very much. 


\section{Contents}

\begin{tabular}{ll}
\hline Abstract & 21
\end{tabular}

\begin{tabular}{ll}
\hline Declaration & 23
\end{tabular}

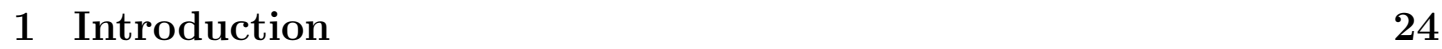

\begin{tabular}{|lll}
2 & History and Theory of Neutrino Physics & 27
\end{tabular}

2.1 Inception and Discovery of the Neutrino . . . . . . . . . . . . . 27

2.2 The Weak Interaction . . . . . . . . . . . . . . . . . . . . . . . . 30

2.3 The Number of Neutrino Generations . . . . . . . . . . . . . . . . 32

2.4 Evidence for Neutrino Oscillations . . . . . . . . . . . . . . . . . 34

2.4 .1 The Solar Neutrino Anomaly . . . . . . . . . . . . . . . . 35

$2.4 .2 \quad$ Atmospheric Neutrino Anomaly . . . . . . . . . . . . . . . 39

2.5 The Theory of Neutrino Oscillations _ . . . . . . . . . . . . . 40

2.5 .1 Three flavor oscillations . . . . . . . . . . . . . . . 43

2.5 .2 The Two Flavor Approximation . . . . . . . . . . . . . . . 44

2.5 .3 Matter Effects . . . . . . . . . . . . . . . 46

2.6 Measurement of the Neutrino Oscillation Mixing Parameters so Far 48

2.6 .1 Solar Neutrino Oscillations . . . . . . . . . . . . . . . . 48

$2.6 .2 \quad$ Atmospheric Neutrino Oscillations . . . . . . . . . . 51

2.6 .3 Reactor Neutrino Oscillations . . . . . . . . . . . 56 
2.7 Ongoing and Future Efforts $\ldots \ldots \ldots \ldots \ldots$

$2.7 .1 \quad$ Majorana Neutrinos $\ldots \ldots \ldots \ldots$

2.7 .2 The Resolution of the Mass Hierarchy. . . . . . . . . . 58

$2.7 .3 \quad$ Measurement of $\delta_{c p} \ldots \ldots \ldots \ldots \ldots \ldots$

2.7 .4 Sterile Neutrinos $\ldots \ldots \ldots \ldots$

$\begin{array}{lll}3 & \text { The MINOS Experiment } & 63\end{array}$

3.1 Physics Goals . . . . . . . . . . . . . . . . . . . . . . . . . . . . 63

3.2 The NuMI Neutrino Beam . . . . . . . . . . . . . . . . . 65

$3.2 .1 \quad$ Neutrino and Antineutrino Production . . . . . . . . . . 65

3.3 MINOS Detectors $\ldots \ldots \ldots \ldots \ldots$. . . . . . . . . . . . . . 72

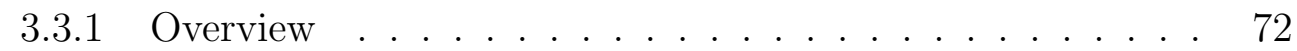

3.3 .2 Steel . . . . . . . . . . . . . . . . . . 75

$3.3 .3 \quad$ Scintillating Strips $\ldots \ldots \ldots \ldots \ldots$. . . . . . . . 75

$3.3 .4 \quad$ Photomultiplier Tubes $\ldots \ldots \ldots \ldots$

$3.3 .5 \quad$ Magnetic Field $\ldots \ldots \ldots \ldots$. . . . . . . . . . . 81

$3.3 .6 \quad$ Electronics and Readout . . . . . . . . . . . . . . . . . 83

3.4 Light Injection $\ldots \ldots \ldots \ldots$

3.5 Triggering $\ldots \ldots \ldots \ldots \ldots \ldots$

3.6 Calibration . . . . . . . . . . . . . . 86

$3.6 .1 \quad$ Absolute Track and Shower Energy _. . . . . . . . . . . 91

3.7 Simulation . . . . . . . . . . . . . . . . . . . . . . . . . . . 91

3.7 .1 Beam Simulation . . . . . . . . . . . . . . . . . . . 91

3.7 .2 Detector Simulation . . . . . . . . . . . . . . . . 93

3.8 Neutrino Events in the MINOS Detectors . . . . . . . . . . . . 95

$3.8 .1 \quad$ Event Topologies . . . . . . . . . . . . . . . . . . . . . 95

3.8 .2 Reconstruction . . . . . . . . . . . . . . . . . . 97 
4 Studies of Muon Neutrino Disappearance through Oscillations $\begin{array}{ll}\text { at MINOS } & 101\end{array}$

4.1 Event Selection . . . . . . . . . . . . . . . . . . . . . . . 102

$4.1 .1 \quad$ Preselection . . . . . . . . . . . . . . . . . . . . . . 103

$4.1 .2 \quad$ Analysis Selection . . . . . . . . . . . . . . . . . 107

4.2 Extrapolation . . . . . . . . . . . . . . . . . . . . . . 115

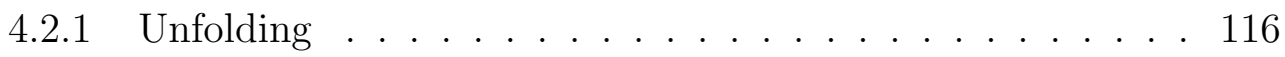

$4.2 .2 \quad$ Beam Matrix . . . . . . . . . . . . . . . . . . . . . 119

4.3 Systematics . . . . . . . . . . . . . . . . . . . . . . . . . . . . 121

4.3.1 Evaluating the Impact Of Different Systematics . . . . . . 124

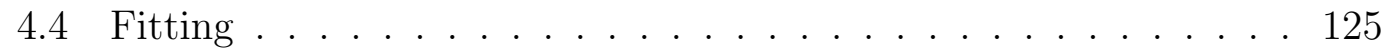

$4.4 .1 \quad$ Systematic Uncertainty . . . . . . . . . . . . . . . . . . . . . 129

$\begin{array}{lll}5 & \text { The Charged Current Analysis } & 131\end{array}$

$5.1 \quad$ Analysis Outline . . . . . . . . . . . . . . . . 132

5.1 .1 Resolution Binning . . . . . . . . . . . . . . . . . . 132

5.1 .2 The Rock and Antifiducial Sample . . . . . . . . . . . . . 134

5.1 .3 Addition of the ND Coil Hole Selection Criteria . . . . . . 136

5.2 Data Epochs . . . . . . . . . . . . . . . . . . . . . . 137

$5.2 .1 \quad$ MC Reweighting . . . . . . . . . . . . . . . . . . 139

$5.3 \quad$ CC Analysis Fit . . . . . . . . . . . . . . . . . . . . . . . . . 141

5.3 .1 Result . . . . . . . . . . . . . . . . . . . . . . . . 142

\begin{tabular}{llr}
\hline 6 & Three Flavour Oscillations & 148
\end{tabular}

$6.1 \quad$ Analysis Overview $\ldots \ldots \ldots$. . . . . . . . . . . . . . . . . 148

6.2 The Exact Three Flavour Oscillation Formula $\ldots . . . . . .149$

6.3 New Truth Binning . . . . . . . . . . . . . . . . . . . . . 150

6.4 Fitting Choices . . . . . . . . . . . . . . . . . 152 
$6.4 .1 \quad$ Octant Dependency . . . . . . . . . . . . . . . . . . 152

$6.4 .2 \quad$ The Effective and Exact Mass Splitting . . . . . . . . . . 154

$6.4 .3 \quad$ Matter Effects and The Density of the Crust . . . . . . . . 154

$6.4 .4 \quad$ Fixing or Floating Mixing Parameters _. . . . . . . . . 154

6.5 Result . . . . . . . . . . . . . . . . . . . . . . . 157

\begin{tabular}{lll}
\hline & Simulating the NuMI Beam & 161
\end{tabular}

$7.1 \quad$ Flux Prediction . . . . . . . . . . . . . . . . . . . . . . . . . . . 162

7.1 .1 Coordinate Transformations . . . . . . . . . . . . . . 162

7.2 Reweighting . . . . . . . . . . . . . . . . . . . 163

$7.2 .1 \quad$ Neutrino Energy Estimation . . . . . . . . . . . . . . . 164

$7.2 .2 \quad$ Decay Probability . . . . . . . . . . . . . . . . . . 165

7.3 Predicted Spectrum $\ldots \ldots \ldots \ldots$

7.3.1 Cross Sections and Neutrino Oscillations . . . . . . . . . . 167

$7.3 .2 \quad$ Peak Finding $\ldots \ldots \ldots \ldots \ldots$

7.4 Mapping the Beam . . . . . . . . . . . . . . . . . . . . . 169

$\begin{array}{lll}8 & \text { Summary } & 171\end{array}$

$\begin{array}{lll}9 & \text { Appendix } & 175\end{array}$

9.1 Deriving Matter Density in the NuMI Beam . . . . . . . . . 175

9.1.1 Average Depth of the NuMI Beam . . . . . . . . . . . 175

9.1 .2 The Density of the Crust . . . . . . . . . . . . . . . . 177

9.2 Summary of Personal Contributions $\ldots \ldots \ldots \ldots \ldots$

\begin{tabular}{ll}
\hline Bibliography & 180
\end{tabular}

Total word count: 23,804 


\section{List of Tables}

3.1 Perecentage contribution of different decays in the target hall to the neutrino event rate in the MINOS Near Detector during low energy forward horn current running after the addition of helium to the pipe. Numbers from truth information in Monte Carlo (MC). 70

$4.1 \Delta \chi^{2}$ at different confidence levels for a one or two dimensional likelihood surface. . . . . . . . . . . . . . . . . . . . . 128

5.1 Run Periods showing accumulated PoT in the Far Detector. Beam types as described in Section 3.2. . . . . . . . . . . . . . . . . . 139

5.2 Run Periods with extra information about conditions during the runs. . . . . . . . . . . . . . . . . . . . 140

5.3 Expected and observed CC analysis Event Counts in the MINOS Far Detector. The oscillated scenario is at the neutrino best fit point. . . . . . . . . . . . . . . . . 147

$6.1 \quad$ Three leading measurements of $\sin ^{2}\left(\theta_{13}\right)$ from Day Bay, Reno, and Double Chooz. . . . . . . . . . . . . . . . . . 158

6.2 Best fits to the mixing parameters allowed to float in our fit for each combination of mass hiearchy and octant. . . . . . . . . . . . 159 


\section{List of Figures}

2.1 The two weak interactions involving neutrinos, a CC interaction on the left and a NC interaction on the right. . . . . . . . . . . 32

2.2 The early understanding of $\beta$ decay left, and on the right with the W boson . . . . . . . . . . . . . . . . . . . 32

2.3 Cross section for $e^{+} e^{-} \rightarrow$ hadrons at center of mass energies near the Z-mass. The prediction for three neutrino flavors (green) clearly fits the data far better than two or four. . . . . . . . . . . . 34

2.4 The solar neutrino spectrum predicted by the BSO5(OP) SSM. Neutrino fluxes from each solar neutrino source are shown with theoretical errors associated with the BSO5 model. . . . . . . . . 37

2.5 Solar $\nu_{e}$ and $\nu_{\mu}+\nu_{\tau}$ flux as measured by the SNO experiment. Dotted lines show the region where $\nu_{e}+\nu_{\mu}+\nu_{\tau}$ sums to the SSM prediction. Blue lines show the region where $\nu_{e}+\nu_{\mu}+\nu_{\tau}$ fluxes sum to the total flux measured from the NC interactions. Red lines show measured $\nu_{e}$ flux from the CC interaction with Deuterium. Green lines show electron scattering measurement (some NC, mainly CC). Grey: The Super Kamiokande equivalent of the green region. . . . . . . . . . . . . . . . . . . . . 39 
2.6 A summary of the deficit of $\frac{(\mu / e)_{D A T A}}{(\mu / e)_{M C}}$ as observed in a variety of experiments. Whilst FREJUS and NUSEX are both consistent, within uncertainties, with $R^{\prime}=1$ they are also consistent with $R^{\prime} \approx \frac{2}{3}$. . . . . . . . . . . . . . . . . 40 40

2.7 Shown in black the ratio of measured to expected $\bar{\nu}_{e}$ as a function of distance traveled by the neutrino divided by its energy. The blue line shows the best fit for the oscillation hypothesis. . . . . . 50

2.8 Measurements of the solar mixing parameters from SNO and other radio chemical experiments (black) and KamLAND (colored region). Not only are the contours in good agreement, they also provide complementary information. . . . . . . . . . . . . . 51

2.9 Atmospheric neutrino event rates measures by Super Kamiokande as a function of zenith angle. Shown are $\nu_{e}$ events (left column), $\nu_{\mu}$ events (right column), high energy events (bottom row) and low energy events (top row). Data (black dots), prediction with null oscillation (red) and prediction with the best fit to oscillations (green) is shown for each set of events. . . . . . . . . . . . . . 53

2.10 The ratio of measured atmospheric neutrino events rates to a null oscillation case as a function of the distance traveled divided by the energy of the neutrino. Show is the data (black points), the best fit to oscillation (black line), the best fit to decoherance (red line) and neutrino decay (blue line). . . . . . . . . . . . . . . 54 
2.11 Confidence limits on the parameters $\left|\Delta m^{2}\right|$ and $\sin ^{2} 2 \theta$, assuming equal oscillations for neutrinos and antineutrinos. The black curve gives the $90 \%$ contour obtained from the combined analysis of MINOS accelerator and atmospheric neutrinos. For comparison, the blue curves show the 90\% contours from Super-K (zenith angle analysis indicated by dotted line, L/E analysis indicated by solid line) and the green curve shows the 90\% contour from T2K. . . . 56

2.12 Feynman diagram showing neutrinoless double beta decay. . . . . 58

2.13 MINOS and Bugey combined 90\% and 99\% confidence level limits on the sterile mixing parameter $\sin ^{2} 2 \theta \mu e=4|U e 4|^{2}|U \mu 4|^{2}$, obtained from the individual disappearance limits of each experiment on the size of $|U \mu 4|^{2}$ and $|U e 4|^{2}$, respectively. Regions of parameter space to the right of the red contour are excluded at 90\% CL, while those to the right of the magenta contour are excluded at 99\% CL. The MINOS limits correspond to a $10.71 \times 10^{20}$ POT exposure in neutrino running mode. The Bugey limits are computed from a GLoBES 2012 fit provided by P. Huber. It accounts for the new calculation of reactor fluxes, as described in P. Huber, Phys. Rev. C 85029901 (2011). The MiniBooNE contours are provided by the MiniBooNE Collaboration and were published in A.A. Aguilar-Arevalo et al., Phys. Rev. Lett. 110, 161801

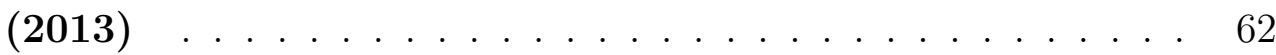

$3.1 \quad$ A sketch showing the baseline of the MINOS experiment and the location of our Near and Far Detectors withing the NuMI beam. . 64

3.2 Schematic of the NuMI Target hall showing an example muon neutrino production via the decay of a focused $\pi^{+}$. . . . . . . . . 66

3.3 Technical drawing of NuMI target and target housing. . . . . . . . 67 
3.4 The transverse $\left(\mathrm{p}_{\mathrm{T}}\right)$ and longitudinal $\left(\mathrm{p}_{\mathrm{Z}}\right)$ momentum distributions of $\pi^{+}$parents of neutrinos (left) and $\pi^{-}$parents of antineutrinos (right) seen at the Near Detector during low energy forward horn current running. The focused component has a range of transverse momentum and longitudinal momentum is peaked at narrow range of energies, the unfocused component has a broad range of $p_{Z}$ and hence total momentum. . . . . . . . . . . . . . . . . . . . . . 69

$3.5 \quad$ The CC $\nu_{\mu}$ (black) and $\bar{\nu}_{\mu}$ (red) event rates at the Near Detector for FHC (left) and RHC (right). . . . . . . . . . . . . . 71

3.6 Scintillator strips attached to steel planes to make a single MINOS plane (left) and with the strips in an alternating $\pm 45^{\circ}$ angle

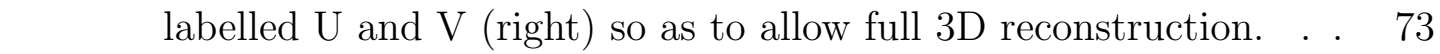

3.7 Technical drawing of an example scintillator strip. . . . . . . . . . 77

3.8 Scintillator module arrangement in the Near Detector planes. The

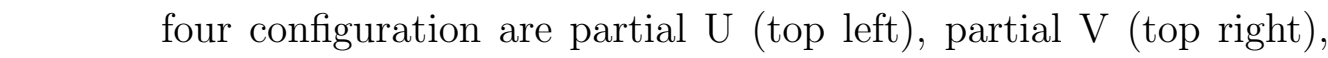
\begin{tabular}{|c|}
\hline full U (bottom left) and full V (bottom right). Every fifth plane \\
\hline
\end{tabular} is alternately a full $\mathrm{U}$ or $\mathrm{V}$ plane. In the upstream calorimeter intervening planes are alternating partial U and V planes with the area near the beam centre, left of the coil hole, instrumented. In the downstream calorimeter the intervening planes are left unistrumented. . . . . . . . . . . . . . . . . . . 78

3.9 Scintillator arrangement in a Far Detector plane. Every plane in the Far Detector is fully instrumented. . . . . . . . . . . . . . . . 78

3.10 Technical drawing showing how WLS fibres from multiple strips are grouped and sent to a multi-pixel photo multiplier tube. In the Far Detector eight strips go to each of 16 PMT pixels, in the Near Detector a single strip goes to each of 64 PMT pixels. . . . . 80 
3.11 Maps of the magnetic field strength in the MINOS Near (left) and Far (right) detectors. Over the fiducial volume the average field strength is $1.42 \mathrm{~T}$ in the Near and $1.28 \mathrm{~T}$ in the Far Detector. . . 82

3.12 Shown is the true energy lost per scintillator plane for a MC cosmic muon and the window in track length used for calibration . . . . . 89

3.13 Data (black), untuned MC (blue) and tuned MC (red) $\nu_{\mu}$ CC spectra at the MINOS Near Detector during a low energy forward horn current run. . . . . . . . . . . . . . . . . . . . . . 93

3.14 On the left a $\mathrm{CC} \nu_{\mu}$ interaction with a clear curving muon track which allows for both $\mathrm{CC} / N C$ identification and distinction between $\mathrm{CC} \nu_{\mu}$ and $\mathrm{CC}_{\mu}$. In the middle a NC interaction characterised by the lack of a track. On the right a $\mathrm{CC} \nu_{\mathrm{e}}$ interaction with a very short $e^{-}$track making it difficult to separate from the NC sample. . . . . . . . . . . . . . . . . . . . . . 96

3.15 Distribution of $E_{\mathrm{reco}} / E_{\text {true }}$ for different ranges of true shower energy. The standard reconstruction is in black, and the kNN energy estimate in red. The dotted lines mark $E_{\text {reco }}=E_{\text {true }}$. The kNN peak is sharper than standard reconstruction at low energies. . . . 100

4.1 Left, Far Detector $\nu_{\mu}$ data and predictions for the no oscillations hypothesis (red histogram) and with the best-fit oscillation parameters from the best fit to the oscillations hypothesis (blue histogram). The band around the oscillated prediction represents the total systematic uncertainty described in section 4.3.1. Total background in the oscillated prediction is also displayed (gray shaded histogram). Right, ratio of Far Detector $\nu_{\mu}$ data to a null oscillation prediction (black) and a ratio of a Far Detector best-fit to oscillation prediction to a null oscillation prediction (blue). . . . . 102 
4.2 Performance of the current FHC selection in the Near (top) and Far (bottom) Detector, which is essentially a CC selection. The dashed lines show the contamination before selection and the solid show efficiency and contamination after selection. Neutral Current (NC) and Wrong charge Sign (WS) backgrounds are shown seperaretly. 104

4.3 Left, the event spill timing plot before (black) and after (red) the cosmic background has been removed by preselection selection cri\begin{tabular}{|c}
\hline teria. Right, cosine of the angle between the muon track and beam \\
\hline
\end{tabular} direction of $\nu_{\mu}$ events in the Far Detector. The dashed colored his\begin{tabular}{|c|c|c|}
\hline togram represents the Monte Carlo expectation with the best fit \\
\hline
\end{tabular} oscillation parameters, the solid colored histogram represent the no oscillations expectation and black points represent data. MC is PoT normalized to data. Preselection removes events with $\theta<0.6$ to reduce background from cosmic rays. . . . . . . . . . . . . . . . 105

4.4 Track Fit pass efficiency of selected $\nu_{\mu}$ events in the Near Detector. The plot on the left does not have the new ND selection criteria, whilst the plot on the right does. . . . . . . . . . . . . . 106

4.5 Near Detector track end $\mathrm{X}$ position distribution. The red histogram represents the Monte Carlo expectation with systematic uncertainties and black points represent data. The distribution shows the effect of the track end radius selection criteria at $0.6 \mathrm{~m} .106$

4.6 Distribution at the MINOS Near Detector for the four input variable to the RoID CC/NC "kNN" based discriminant. Shown is selected MC (red), data (black), and NC background in the selected MC (blue) with systematic uncertainties on the MC shown by the shaded region. . . . . . . . . . . . . . . . . . . . 109 
4.7 RoID CC/NC separation parameter of events passing all other selection selection criteria. The red histogram represents the Monte Carlo expectation with systematic uncertainties, the blue histogram represents the NC background with systematic uncertainties. Black points represent data. The selection criteria removes events with $\mathrm{PID}<0.3 . \ldots \ldots \ldots \ldots \ldots \ldots$

4.8 Distribution at the MINOS Near Detector for the four input variable to the jmID CC/NC kNN based discriminant. Shown is selected MC (red), data (black), and NC background in the selected \begin{tabular}{|c|}
\hline MC (blue) with systematic uncertainties on the MC shown by the \\
\hline
\end{tabular} shaded region. . . . . . . . . . . . . . . . . . . . 112

4.9 Low energy jmID CC/NC separation parameter of events passing all other selection selection criteria. The red histogram represents the Monte Carlo expectation with systematic uncertainties, the blue histogram represents the NC background with systematic uncertainties. Black points represent data. The selection criteria removes events with PID $<0.5$.

$4.10(q / p)$ distribution of events passing all selection selection criteria in the Near Detector. The red curve represents MC expectation with the systematic uncertainty while the black dots represent data. 114

$4.11(q / p) / \sigma(q / p)$ (track charge sign significance) distribution of selected antineutrino events in the Near Detector. The red curve represents MC expectation with the systematic uncertainty, the blue curve represents the $\mathrm{CC}$ and NC backgrounds with systematic errors and black dots represent data. . . . . . . . . . . . . . 115

4.12 The steps in the extrapolation of a Near Detector measurement to a Far Detector prediction. . . . . . . . . . . . . . . . 116 
4.13 Purity as a function of reconstructed energy at the Near (left) and Far (right) MINOS detectors. . . . . . . . . . . . . . . . . . . . . 117

4.14 True vs. Reconstructed energy at the Near (left) and Far (right) MINOS detectors. . . . . . . . . . . . . . . . . . . . . . . 117

4.15 Efficiency as a function of true energy at the Near (left) and Far (right) MINOS detectors. . . . . . . . . . . . . . . . . . . 118

4.16 Example muon neutrino energy spectrum at the Near (left) and Far (right) MINOS Detectors. The coloured regions in each plot correspond to the same neutrino parent meson decay positions and momenta. Differences in geometric acceptance at the detectors leads to both the smearing of this highlighted regions and the differences between the two spectra. . . . . . . . . . . . . . . . . . . 119

4.17 Beam matrix used to convert a neutrino Near Detector flux into a Far Detector flux. . . . . . . . . . . . . . . . . . . . . . . 120

4.18 The shifts to the best fit oscillation parameters induced by the application of the 2011 NuMuBar RHC analysis (top) and 2012 NuMu FHC analysis (bottom) systematic shifts to the "fake data". The sum, in quadrature, of all cross section shifts is also shown. . 126

4.19 The shifts to the best fit oscillation parameters overlaid on the statistical sensitivity contour for the RHC (left) and FHC (right) neutrino analyses. . . . . . . . . . . . . . . . . . . 127

4.20 The energy dependant systematic uncertainty at the Near Detector (left) and Far Detector (right). . . . . . . . . . . . . . . . . . . . . 127 
4.21 The distribution of the major systematics obtained by a two parameter fit on 1000 fake experiments using MINOS Beam Monte Carlo data sets. The distributions of best fit systematics all have a mean value close to zero and width close to unity, and are welldescribed by Gaussian distributions, indicating no apparent bias or pathology in the two parameter fit. . . . . . . . . . . . . . . . . 129

$5.1 \sigma_{\text {total }} /$ Reconstructed Energy, the energy resolution as a fraction of total reconstructed energy, vs. the reconstructed energy of selected events for the null oscillation case at the Far Detector. The boundaries between the five energy resolution quantiles are shown in black and each bin of resolution contains the same number of events. Quasi-elastic events with zero shower energy appear as a band of events at the low edge of the distribution. . . . . . . . . . 133

5.2 The Reconstructed vs. True energy matrices for the highest (left) and lowest (right) energy resolution quantiles. . . . . . . . . . . . 134

5.3 True interaction vertices of events selected into the RAF sample, taken from MC. Both the structure of the cavern and the supermodules is visible, with the majority of events coming from rock interactions upstream of the detector. . . . . . . . . . . . . 136

5.4 Scale diagram showing the detector regions (coloured) used to separate events in the RAF sample . . . . . . . . . . . . . . . . . . 136

5.5 Predicted spectrum of selected $\nu_{\mu}$ events in the Far Detector (left), ratio of spectrum as measured by different analyses (right). . . . . 137

5.6 The major sources of uncertainty in the 2010 (top) and 2012 analysis (bottom). . . . . . . . . . . . . . . . . . . 138

5.7 Ratio of ND muon neutrino spectrum in Runs 3 and 5 alongside the ratio of the Run $3 \mathrm{MC}$ with and without target decay weighting. 141 
5.8 Reconstructed energy distribution of events selected as neutrinos in the Near Detector. The red histogram represents the Monte Carlo expectation with the systematic error, the blue histogram represents the total neutral current background, and black points represent data. . . . . . . . . . . . . . . . . . . 143

5.9 Far Detector data and predictions with no oscillations (red histogram) and with the best-fit oscillation parameters (blue histogram). The band around the oscillated prediction represents the total systematic uncertainty. Total background in the oscillated prediction is also displayed (gray shaded histogram). The data and predictions correspond to an exposure of $10.7 \times 10^{20}$ PoT. 144

5.10 Ratio of Far Detector data and best-fit prediction to no oscillations. The data and predictions correspond to an exposure of $10.7 \times$ $10^{20} \mathrm{PoT} \ldots \ldots \ldots \ldots \ldots \ldots \ldots \ldots$

5.12 1D marginalizations of our full contour for $\Delta \chi^{2}$ for $\Delta \mid m_{a m}^{2}$ (left) and $\sin ^{2} 2 \theta_{23}$ (right). Dashed lines show $68 \%$ and 90\% C.L. . . . . 146

5.11 The 90\% 2012 CC Analysis contour from FHC running. The best fit is at $\Delta\left|m_{\text {atm }}^{2}\right|=2.42 \times 10^{-3} \mathrm{eV}^{2}, \sin ^{2}\left(2 \theta_{23}\right)=0.936 \ldots$. . . 146

6.1 On the left is the absolute difference between two flavour approximate and three flavour exact oscillation probabilities as a function of length and energy. On the right the difference as a function of energy for the MINOS baseline of $735 \mathrm{~km}$. Nonzero $\theta_{13}$ and maximal $\theta_{23}$ are assumed. . . . . . . . . . . . . . . . . . 150 
6.2 Predicted and fake data Far Detector Spectra (left) and the ratio of fake data over prediction (right) for oscillated Far Detector MC.

Not that the finer binning and no interpolation (blue) is equivalent to the combination of fine binning and higher order interpolation (red).

6.3 Oscillated FD Fake Data and predicted reconstructed energy spec-

\begin{tabular}{|c|c|}
\hline tra for the old coarse and the new fine truth binnings left, ratios \\
\hline
\end{tabular} of predictions against fake data on the right. . . . . . . . . . . . . 152

6.4 The plot on the left shows $-2 \Delta \log (L)$ as a function of $\sin ^{2}\left(\theta_{23}\right)$ and $\sin ^{2}\left(\theta_{\text {eff }}\right)$. On the right $-2 \Delta \log (L)$ as a function of $\sin ^{2}\left(2 \theta_{23}\right)$

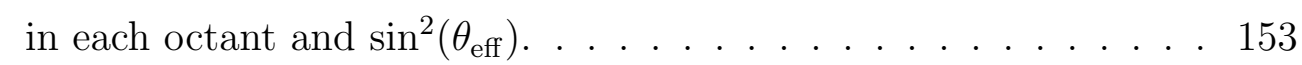

6.5 The plot on the left shows $90 \%$ C.L. contours for $\Delta m_{32}^{2}$ and $\sin ^{2}(2 \theta)$. The plot on the right shows muon neutrino survival probability as \begin{tabular}{|c|c|c|}
\hline a function of $\sin ^{2}\left(2 \theta_{23}\right)$ and $\sin ^{2}\left(2 \theta_{\text {eff }}\right)$. The different size contours \\
\hline
\end{tabular} in the left most plot is a direct consequence of the different octant oscillation probabilities in the rightmost plot. . . . . . . . . . . . 153

6.6 A starplot showing the effect on our best fit as we vary $\theta_{13}, \theta_{12}$, and $\Delta\left|m_{21}\right|^{2}$ over their PDG uncertainties and as we vary $\delta_{c p}$ over 0 to $2 \pi \ldots \ldots \ldots \ldots \ldots \ldots \ldots$

6.7 A starplot showing in contrast the effect of systematics, the solar and reactor parameters have been set to bright green to make their relative size clear. . . . . . . . . . . . . . . . . . . . . . 155

6.8 The best fit to $\sin ^{2}\left(\theta_{13}\right)$ as a function of $\theta_{23}$ and $\Delta m_{\text {atm }}^{2}$ for the normal hiearchy on the left and the inverted on the right. . . . . . 156

6.9 The best fit of $\delta_{c p}$ as a function of $\theta_{23}$ and $\Delta m_{a t m}^{2}$ for the normal hiearchy on the left and the inverted on the right. . . . . . . . . . 156 
$6.1090 \%$ C.L. for both normal and inverted hiearchy with best fit points for the lowest $\log (L)$ in each combination of octant and hiearchy. . 159

6.11 1D marginalizations of our full contour for $\Delta \chi^{2}$ for $\Delta m_{32}^{2}$ (left) and $\sin ^{2} \theta_{23}$ (right). Show are both the normal (red) and inverted (blue) mass hiearchies. Dashed lines show 68\% and 90\% C.L. . . . 160

7.1 Neutrino flux as a function of energy at the MINOS, $\mathrm{NO} \nu \mathrm{A}$, and CHIPS Far Detector locations assuming null oscillations. . . . . . 167

7.2 A spline of CC cross sections as a function of energy. . . . . . . . 168

7.3 On the left are comparative muon neutrino true energy spectrums distributions at the MINOS, $\mathrm{NO} \nu \mathrm{A}$, and CHIPS Far Detector locations assuming null oscillations and one kiloton-year of running. On the right is the true muon neutrino event spectrum that would be seen at CHIPS in one kiloton-year with (red) and without (black) neutrino oscillations. . . . . . . . . . . . . . . . . . . 169

$7.4 \quad$ A spectrum for a hypothetical detector location in the Mesabi range(black line) with the peak estimation shown (red dot). . . . 169

7.5 A map of potential neutrino event rates, assuming a null oscillations hypothesis, in the $0-30 \mathrm{GeV}$ region as seen within the Mesabi range within one Kiloton year. Contours show lines of constant $\mathrm{L} / \mathrm{E}$ where $\mathrm{L}$ is the distance from the hypothetical detector to the NuMI target and $\mathrm{E}$ is the peak energy of the reweighted neutrino spectrum. . . . . . . . . . . . . . . . . 170 
8.1 The $68 \%$ and $90 \%$ confidence limits for the $\left(\Delta m_{32}^{2}, \sin ^{2} \theta_{23}\right)$ parameter space, resulting from a combined fit to the MINOS $\nu_{\mu}$ disappearance and $\nu_{e}$ appearance data. The best fit occurs in the inverted hierarchy and lower octant at $\left(\Delta m_{32}^{2} / \mathrm{eV}^{2}, \sin ^{2} \theta_{23}\right)=$ $\left(-2.41 \times 10^{-3}, 0.41\right)$, as indicated by the star. For both the normal and inverted hierarchy, the $-\Delta \log L$ surface is calculated relative to this overall best fit point. . . . . . . . . . . . . . . . 174

$9.1 \quad$ A diagram showing the simplified NuMI geometry we are about use.176 


\section{Abstract}

Name of the University: University College London

Candidate Name: Alexander Radovic

Degree Title: Doctor of Philosophy in the Faculty of Physical Sciences

Thesis Title: Measuring the Disappearance of Muon Neutrinos with the MINOS Detectors

Date: August 2013

MINOS is a long baseline neutrino oscillation experiment. It measures the flux from the predominately muon neutrino NuMI beam first $1 \mathrm{~km}$ from beam start and then again $735 \mathrm{~km}$ later using a pair of steel scintillator tracking calorimeters. The comparison of measured neutrino energy spectra at our Far Detector with the prediction based on our Near Detector measurement allows for a measurement of the parameters which define neutrino oscillations. This thesis will describe the most recent measurement of muon neutrino disappearance in the NuMI muon neutrino beam using the MINOS experiment.

The general method of a disappearance analysis at the MINOS experiment will be outlined, the selection of events, extrapolation between detectors, and fitting the data to the atmospheric mixing parameters. An analysis of the full MINOS Forward Horn Current charged current muon neutrino interactions sample is detailed, with a best fit to the atmospheric mixing parameters in a two flavour 
approximation of $\Delta\left|m_{\text {atm }}^{2}\right|=2.42 \times 10^{-3} \mathrm{eV}^{2}$ and $\sin ^{2}\left(2 \theta_{23}\right)=0.936$. The change to a three flavor analysis with matter effects from a simple two flavour approximation is described, with a very slight preference of $-2 \Delta \log (L)=0.01$ for the inverted hiearchy found. A study of the NuMI beam is also shown, with potential locations for new oscillation experiments in the NuMI beam discussed. 


\title{
Declaration
}

No portion of the work referred to in this thesis has been submitted in support of an application for another degree or qualification of this or any other university or other institution of learning.

\author{
Alexander Radovic \\ Department of Physics and Astronomy \\ University College London \\ Gower Street \\ London \\ WC1E 6BT
}

August 2013 


\section{Chapter 1}

\section{Introduction}

Originaly conceived of as a solution to the problem of missing energy in $\beta$ decay, neutrinos have proven to be a fascinating source of new physics. Their interaction through only the weak force creates unique difficulties in their study, though this very property has given us powerful insight into the behaviour of weak interactions. Most recently the spontaneous flavour changing of massive neutrinos through neutrino oscillations has given us a rare window into beyond the Standard Model physics. The precise measurement of the parameters describing this mixing is a vibrant field with a rich history. It is to this field that this thesis seeks to contribute.

Chapter 2 of this thesis will give an overview of the history and theory of high energy particle physics as it pertains to neutrinos. Starting with its original conception, continuing through to the discovery of neutrino oscillations, the theory of three flavour neutrino oscillations in matter, and finishing with a summary of of the final state and future prospects for the measurement of the PMNS matrix (described in Section 2.5.1).

MINOS is a long baseline experiment in the NuMI beam designed to measure 
the atmospheric components of the PMNS matrix. Chapter 3 will describe in some detail the construction and modus operandi of the MINOS experiment and detectors. The chapter begins with an outline of the physics goals of the experiment, following with a description of the NuMI neutrino beam, the MINOS detectors, the methodology of our MC, calibration, and finishing with the reconstruction of the neutrino $\mathrm{CC}$ and $\mathrm{NC}$ events which will be vital to the following chapters.

In Chapter 4 the general method of a muon neutrino disappearance analysis at the MINOS experiment will be outlined. Starting with the selection of events, continuing with a detailed outline of extrapolation between detectors, and ending with a description of how we fit our observed muon neutrino spectra to the atmospheric mixing parameters.

Chapter 5 builds on the general technique described in Chapter 4 by describing in detail the analysis of charged current muon neutrino interactions from the NuMI beam during forward horn current running. Particular fitting techniques, data samples, and the distinct periods of data taking used are discussed.

Motivated by the recent measurement of a large $\theta_{13}$ by Daya Bay the latest version of the MINOS CC analysis makes use of a full three flavour with matter effects model of neutrino oscillations. Chapter 6 describes in detail both the changes to the fit parameters and subtle changes to the analysis technique which this requires.

As well as supporting the MINOS experiment the NuMI beam hosts a number of current and future experiments. Chapter 7 of this thesis will discuss a 
study of neutrino flux from NuMI in the Mesabi range.

Finally Chapter 8 of this thesis summarises the results of this thesis and the prospects for the field as a whole. 


\section{Chapter 2}

\section{History and Theory of Neutrino Physics}

This chapter deals with the history and theory of high energy particle physics as it pertains to neutrinos. It begins with a discussion of the neutrino's inception as a solution to the problem of continuous spectrum from $\beta$ decay and its role in the weak force in 2.1-2.3. The anomalous atmospheric and solar measurements that lead to the formulation of the theory of neutrino oscillations are described in 2.4. The theory of neutrino oscillations, both in a two flavor approximation and in the exact three flavor treatment with matter effects is discussed in 2.5. Finally the current state and future prospects for the measurement of the PMNS matrix characteristics is described in section 2.6-2.7.

\subsection{Inception and Discovery of the Neutrino}

To explain the need for the neutrino we must first return to a problem that deeply troubled the field of particle physics in the early 20th century, that of the continuous spectrum of $\beta$ decay. At the time the postulate was that the electron 
released in the $\beta$ decay of a particular atom should have a unique discrete energy rather than a continuous energy. This is a very reasonable assumption as after the description of the photoelectric effect by Einstein in 1908 [1] you might analogously expect the atom's quantised energy levels to give rise to decays with defined and distinct energy distributions.

However in 1908 James Chadwick [2] showed that the energy spectrum was in fact continuous rather than discrete. Several attempts were made to try and explain how a continuous signal might come from a discrete decay. Some were fairly reasonable, including the idea that the electron was losing energy into the medium containing the radioactive nuclei- disproved by the measurements of Ellis and Wooster in 1927 [3] which showed that the energy deposited in a calorimeter surrounding a beta source did not sum to that of the highest energy electron observed but rather was consistent with the mean of the previously measured spectrum. Others, including Niels Bohr [4], even suggested that energy might not be conserved in individual decays.

The solution came in the form of an open letter from Wolfgang Pauli [5] where he described a "desperate remedy to save the "exchange theorem" of statistics and the laws of conservation of energy.". In this letter Pauli proposed the existence of a new particle called the Neutronen, which had both spin $\frac{1}{2}$ and charge 0 . He suggested that not only could this undetectable particle carry off the missing energy, it could also address the problem of integer spin of Nuclei such as Nitrogen where the current model at the time was 14 protons and 7 electrons in the nucleus to give the known mass and charge but the wrong (non integer spin). In effect Pauli in one letter proposed the existence of not just the neutrino but also the neutron, combined in the one neutronen. 
The divergence of those two concepts came in 1932 with the discovery of the neutron by Chadwick [6]. Whilst studying the radiation given off by Beryllium and Boron he found that it was far too penetrating to be a photon. Chadwick concluded that the radiation was formed of neutral particles slightly heavier than the proton, neutrons.

The neutrino itself was born as part of the first successful theory of $\beta$ decay put forward by Enrico Fermi in 1933. Constructed in a way analogous to the theory of electromagnetic interactions [7] 8 ] the theory used both Chadwick's and Pauli's Neutrons. However he showed that to achieve the observed $\beta$ decay spectrum the mass of Pauli's neutron must be incredibly small and coined the name neutrino or "little neutral one" to distinguish it from Chadwick's more massive neutron. In this theory both the electron and the neutrino are produced during the decay of a neutron into a proton.

This work also helped to explain why the neutrino had not yet been discovered. Hans Bethe and Rudolf Peierls [9] were able to use the theory to calculate the cross section for the neutrino to interact with a nucleus, and found it to be so incredibly small that it was dismissed as too small to ever be detected.

In fact it wasn't until the 1950's that the study of neutrinos was made possible by the rise of commercial nuclear fission reactors where a suitably high flux of neutrinos was isotropically produced in many of the nuclear reactions taking place in its core. The actual discovery of the neutrino itself was made at the Hanford reactor in 1953 by Fred Reines and Clyde Cowan [10]. They placed a 300 litre tank of cadmium doped liquid scintillator, surrounded by photomultiplier 
tubes (PMTs), next to a reactor. The signal was the annihilation of positrons created by the collision of neutrinos with protons in the tanks $\bar{\nu}_{e} p \rightarrow n e^{+}$which produced two prompt photons. This experiment, together with the later and more ambitious work of Reines and Cowan at the Savannah River Plan in 1956 [11] represented the first conclusive detection of the neutrino.

\subsection{The Weak Interaction}

The development of the weak interaction begins with Fermi's $\beta$-decay matrix. Taking the form:

$$
G_{F}\left(\bar{u}_{n} \gamma^{\mu} u_{p}\right)\left(\bar{u}_{\nu} \gamma_{\mu} u_{e}\right)
$$

where $G_{F}$ is the coupling constant, $u_{x}$ is the Dirac spinor of the particle, and the $\gamma_{\mu}$ are the Dirac gamma matrices. At this stage it contained no propagator and assumed parity conservation.

The Fermi formulation had two problems. The first was that in 1936, Heisenberg [12] had realized that the Fermi theory predicted a cross section of $\sigma(\nu) \propto E_{\nu}^{2}$ ( $E_{\nu}^{2}$ being neutrino energy) which violated unitary at around $300 \mathrm{GeV}$.

The second was the assumption that weak interactions conserved parity. This assumption was shown to be false by the study of two very similar particles named at the time $\theta^{+}$and $\tau^{+}$which decayed respectively via two positive parity or three negative parity pions. These two particles had also been shown to have very similar lifetimes and masses. This strange coincidence prompted Tsung-Dao Lee and Chen-Ning Yang to review the experimental evidence for parity conservation [13]. Not only did this review lead them to suggest there was no good evidence for parity conservation outside of the strong and electromagnetic interactions, but they therefore suggested that the $\theta^{+}$and $\tau^{+}$were in fact one and the 
same particles decaying to different parity states.

Later that same year Chen-Shiun Wu performed an experiment [14] which observed $\beta$ decay from a low temperature magnetically aligned sample of ${ }^{60}$ Co which showed the $\beta$ decay electrons had been emitted preferentially in the opposite direction to the nuclear spin. This provided direct evidence for parity violation and a justification for $\theta^{+}$and $\tau^{+}$being viewed as two decay channels of a new particle called the $K^{+}$. It also undermined the current formulation of Fermi's $\beta$-decay matrix.

These problems motivated two profound changes. To solve the problem of unitarity violation at $300 \mathrm{GeV}$ the concept of exchange bosons, as already introduced to the strong force by Yukawa [15], was applied to the weak interaction.

Any interaction mediated by a spin-1 exchange boson can have a vector or axial nature. Interactions which are purely axial or purely vector couple identically to right and left handed particles. However interactions with a combination of the two violate parity. To account for the discovery of parity violation, the vector only nature of the force was questioned and in 1958 [16][17] an equal axial component $\left(\gamma_{\mu} \gamma_{5}\right)$ was included in the weak current to give a matrix element of:

$$
\frac{G}{\sqrt{2}}\left(\bar{u}_{n} \gamma^{\mu}\left(1-\gamma_{5}\right) u_{p}\right)\left(\bar{u}_{\nu} \gamma_{\mu}\left(1-\gamma_{5}\right) u_{e}\right)
$$

However it wasn't until the 1960's that the weak exchange boson was formally included by Sheldon Glashow, Abdus Salam, and Steve Weinberg (GSW) [18] [19] [20] as part of the unification of the weak and electromagnetic forces. The same theory also predicted the "neutral-current" component of the weak force and masses for the exchange bosons including both charged $\mathrm{W}$ bosons and the neutral Z boson. Neutral currents were observed in 1973 by the Gargamelle bubble chamber 

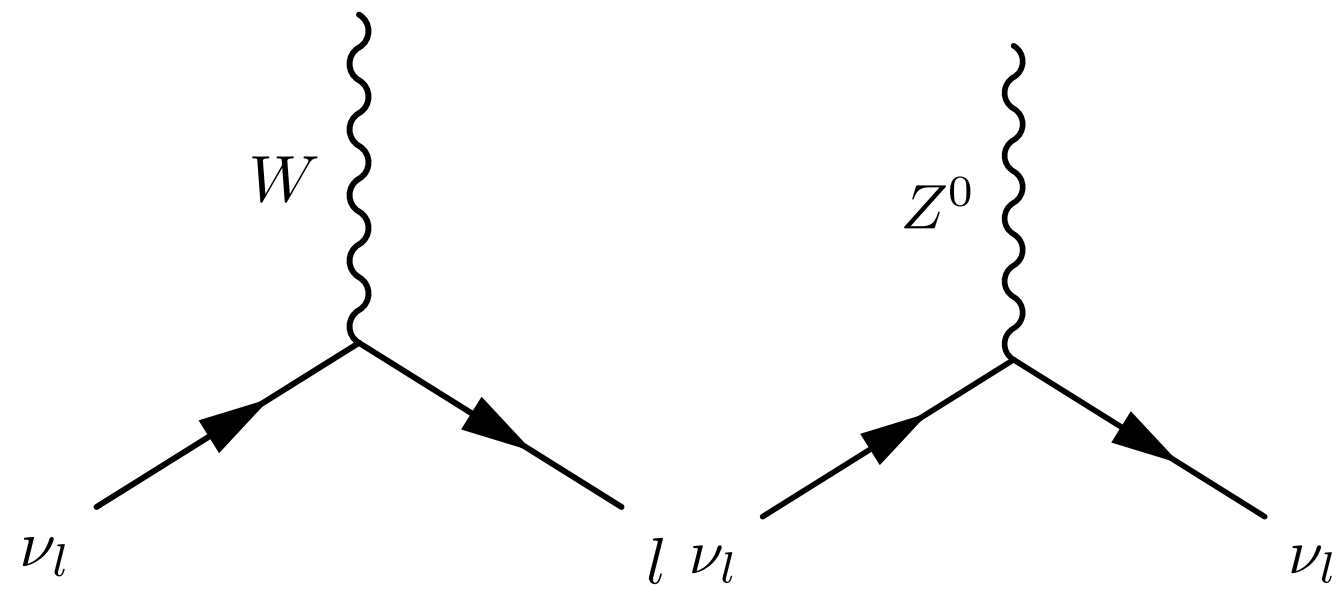

Figure 2.1: The two weak interactions involving neutrinos, a CC interaction on the left and a $\mathrm{NC}$ interaction on the right.

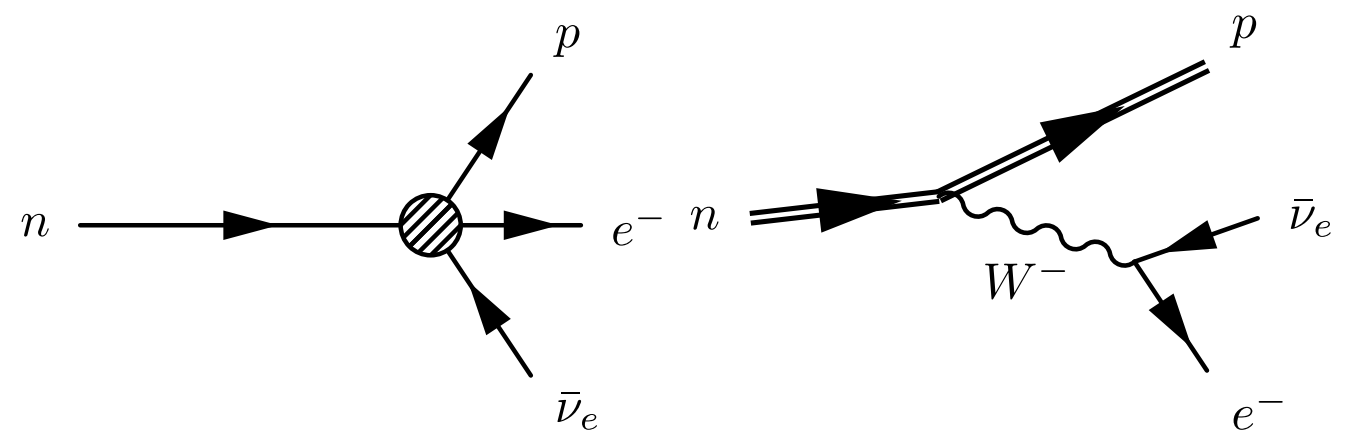

Figure 2.2: The early understanding of $\beta$ decay left, and on the right with the W boson

at CERN [21] 22] and direct discovery of the exchange bosons was made in 1983 by the UA1 and UA2 CERN experiments [23] 24] [25][26]. Charged Current (CC) and Neutral Current (NC) neutrino interactions can be seen ind Figure 2.1 and the fully developed understanding of beta decay can be seen in Figure 2.2 .

\subsection{The Number of Neutrino Generations}

Whilst the theory of lepton number was first introduced in 1953, it wasn't until an experiment in Brookhaven in 1962 that it was demonstrated to exist [27]. It was shown that neutrinos produced in the decays of pions would interact to produce muons rather than electrons, giving the first clear evidence for two families of 
neutrinos- one coupling to the electron and the other the muon. This, in addition to measurements of $\mu \rightarrow e+$ missing energy, paved the way for the introduction of not just overall lepton number conservation but also conservation for electron and muon lepton flavor separately.

The first evidence for a third generation of leptons came from SLAC in 1975 [28]. The process $e^{+}+e^{-} \rightarrow e^{ \pm}+\mu^{\mp}+[$ missing energy] was observed and interpreted as the the production of a pair of particles decaying to muons or electrons along with the associated neutrinos. Whilst the measurement suggested the existence of a third, $\tau$ lepton, it would take other experiments to provide definitive evidence for the existence of a $\tau$ neutrino.

Those experiments were ALEPH, DELPHI, L3 and OPAL on the LEP Lepton Positron Collider[29], where extremely precise measurements of the Z boson cross section were made. The $\mathrm{Z}$ boson width is sensitive to the number of neutrino flavors as one of the decay modes is $Z^{0} \rightarrow \nu \bar{\nu}$. More flavors means more leptonic decay modes and lower hadronic crossection for the Z Leaving the number of neutrinos as a free parameter in the fit to hadronic cross sections as a function of centre of mass energy, $N_{\nu}=2.9840 \pm 0.0082$ was measured providing the first evidence for a third neutrino generation. Predictions for different numbers of neutrinos compared to data can be seen in Figure 2.3. Additionally the only way for a fourth neutrino in light of this measurement would either be one with mass in excess of half the $\mathrm{Z}$ boson mass $(>45 \mathrm{GeV})$ or if the new generation were "sterile" and did not couple to the Z.

Final, direct, confirmation of the $\nu_{\tau}$ was obtained at the DONUT experiment at Fermilab National Accelerator Laboratory (FNAL) [30]. They used a neutrino 
beam created from charged meson decay, produced by a $800 \mathrm{GeV}$ proton beam impinging on a tungsten target. The detector consisted of interleaved layers of steel and photographic emulsion. Their signal was a track with a sharp kink near the vertex indicating the decay of the shortlived $\tau$. Their final dataset with 9 candidate events and an expected background of 1.5 events [31] gave conclusive evidence for the existence of the $\nu_{\tau}$.

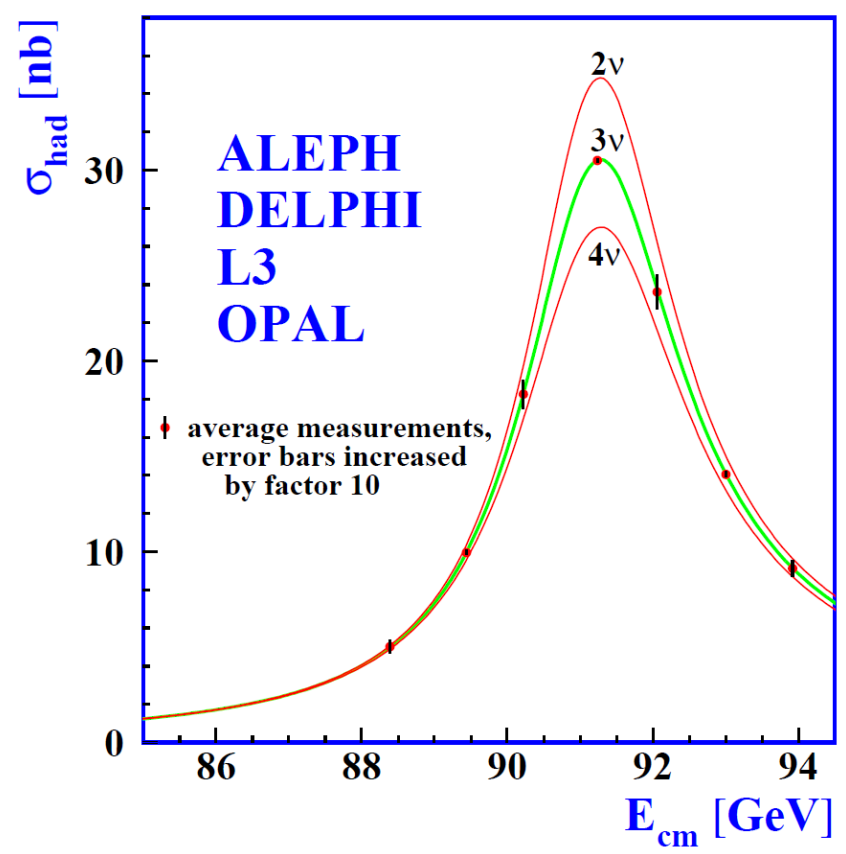

Figure 2.3: Cross section for $e^{+} e^{-} \rightarrow$ hadrons at center of mass energies near the Z-mass. The prediction for three neutrino flavors (green) clearly fits the data far better than two or four.

\subsection{Evidence for Neutrino Oscillations}

The latter part of the 1960s sees the Standard Model neutrino take form. With interactions described by Glashow, Weinberg, and Salam's theory of the electroweak force this neutrino is massless, exists in three distinct lepton flavors, and appears only in a left handed helicity. However during the 1970's an experiment 
designed to measure the flux of neutrinos from the sun and measurements of neutrino flux from cosmics rays hitting the earths atmosphere showed energy dependant deficits which would come to be best explained by the spontaneous change of neutrino flavour states.

\subsubsection{The Solar Neutrino Anomaly}

Whilst theoretical understanding of the processes of fusion and fission in the sun was well developed in 1970, direct confirmation had so far eluded solar scientists. The center of energy production was simply too deep for conventional observation. The solution to this problem came in the form of neutrinos.

The Standard Solar Model (SSM) is composed of two main processes. The pp chain of ${ }_{2}^{2} \mathrm{H}+{ }_{2}^{2} \mathrm{H} \rightarrow{ }_{4}^{4} \mathrm{He}$ followed by ${ }_{2}^{2} \mathrm{He} \rightarrow{ }_{1}^{2} \mathrm{D}+e^{+}+\nu_{e}+0.42 \mathrm{MeV}$ and the carbon/nitrogen/oxygen catalyzed CNO cycle of $4{ }_{1}^{1} \mathrm{H} \rightarrow{ }_{2}^{4} \mathrm{He}+2 e^{+}+\nu_{e}+3 \gamma+$ 26.8 MeV. Both processes produce neutrinos of distinct energies and rates so an experiment that could detect them would be able to provide a strong check of the SSM.

The first observation of these solar neutrinos came from Ray Davis at the Homestake mine in 1968 [32]. Working deep underground in the homestake mine to limit backgrounds from cosmic ray interactions in the upper atmosphere, they used a 390,000 litre container of tetrachloroethylene to search for neutrino oscillations. Their signal came from the production of unstable ${ }^{37} \mathrm{Ar}$ in $\nu_{e}+{ }^{37} \mathrm{Cl} \rightarrow e^{-}+{ }^{37} \mathrm{Ar}$ which was flushed out of the container with helium gas and detected by its decay back to ${ }^{37} \mathrm{Cl}$.

Intriguingly the data from the Homestake experiment consistently showed a 
markedly lower than expected $\nu_{e}$ interaction rate, approximately a third of what they expected from the SSM [33]. Initially it was assumed the cause of this "solar neutrino problem" lay in either some mistake in the SSM or an undiscovered systematic error. Two key measurements proved this hypothesis false.

Firstly in 1989 Kamiokande-II produced a vary similar measurement to that of Davis, now looking for the signal of a solar neutrino interacting with an electron in water via the $\mathrm{NC}$ in the form of Cerenkov light [34. Kamiokande-II saw the same deficit as Ray Davis, and were also able to use the direction of the recoil electron to reconstruct the direction of the original neutrino and confirm the neutrinos were coming from the sun.

Secondly SAGE in 1991 [34] and GALLEX in 1992 [35] provided a measure of very low energy neutrinos via the process $\nu_{e}+{ }^{71} \mathrm{Ga} \rightarrow{ }^{71} \mathrm{Ge}+e^{-}$in a method analogous to Argon production at Homestake. The low energy threshold of this reaction, $233 \mathrm{keV}$, meant that it was sensitive to the first step of the pp chain specifically. As the rate of this reaction is very well constrained by the observed luminosity of the sun the neutrino deficit observed by SAGE and GALLEX gave the first truly compelling evidence that the SSM model was not the culprit, but rather $\nu_{e}$ were somehow disappearing in transit from the sun. Figure 2.4 shows the solar neutrino flux broken down into seperate neutrino sources. 


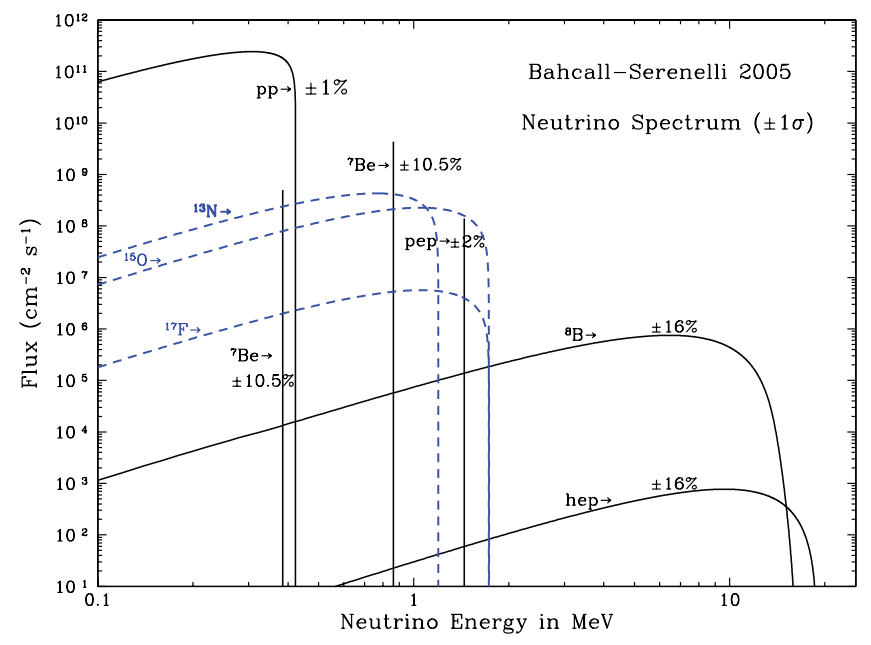

Figure 2.4: The solar neutrino spectrum predicted by the BSO5(OP)[36] SSM. Neutrino fluxes from each solar neutrino source are shown with theoretical errors associated with the BSO5 model.

Definitive proof that the SSM was correct and that electron neutrinos were instead disappearing through the phenomena of neutrino oscillations between lepton flavors came from the Sudbury Neutrino Observatory (SNO). Consisting of 1000 tons of heavy water $\left(\mathrm{D}_{2} \mathrm{O}\right)$ in a spherical vessel, surrounded by PMTs and deep underground SNO was able to measure three distinct kinds of neutrino interactions:

-CC interaction: $\nu_{e}+d \rightarrow p+p+e^{-}$, sensitive only to electron neutrinos.

$\bullet \mathrm{NC}: \nu_{x}+d \rightarrow p+n+\nu_{x}$, sensitive to all flavors equally.

-Elastic Scattering: $\nu_{x}+e^{-} \rightarrow \nu_{x}+e^{-}$, sensitive to all flavors through NC interactions, though predominately $\nu_{e}$ as they have an additional CC s channel.

Enhancement of the NC signal was achieved by doping the heavy water with $\mathrm{NaCl}$. This had two key effects: the photons emitted in neutrino capture on chlorine are more energetic than deuterium which separates them from low energy 
backgrounds; the isotropy of the several photons emitted contrasts very clearly with the single electron from charge current interactions/elastic scattering allowing good separation from the CC signal.

It was this sensitivity to NC interactions which allowed SNO to solve the Solar Anomaly as they were able to measure the solar neutrino flux independenty of any oscillations which may have occurred. This total flux of solar neutrinos was consistent with the overall neutrino flux predicted by the SSM [37]. Fitting to all the data they get a non-zero flux of non-electron-flavor neutrinos combined with an overall rate that is consistent with the SSM prediction. The conclusion of the community was that the SSM prediction of $\nu_{e}$ yield was correct but that approximately two thirds of these converted into some combination of $\nu_{\mu}$ and $\nu_{\tau}$ in the long journey to earth. Figure 2.5 shows how the different channels come together to form a picture of neutrino oscillations. 


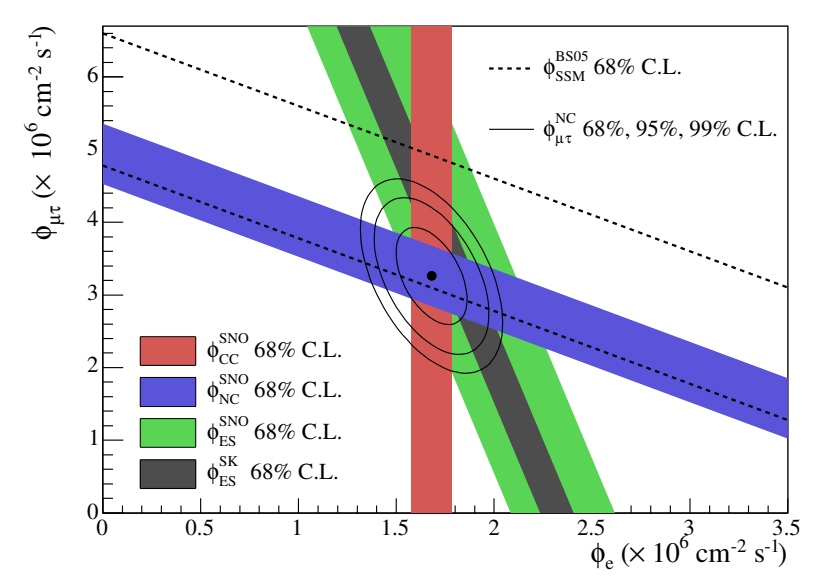

Figure 2.5: Solar $\nu_{e}$ and $\nu_{\mu}+\nu_{\tau}$ flux as measured by the SNO experiment. Dotted lines shows the region where $\nu_{e}+\nu_{\mu}+\nu_{\tau}$ sums to the SSM prediction. Blue lines show the region where $\nu_{e}+\nu_{\mu}+\nu_{\tau}$ fluxes sum to the total flux measured from the $\mathrm{NC}$ interactions. Red lines show measured $\nu_{e}$ flux from the CC interaction with Deuterium. Green lines show electron scattering measurement (some NC, mainly CC). Grey: The Super Kamiokande equivalent of the green region. Figure from [37].

\subsubsection{Atmospheric Neutrino Anomaly}

During the same period an anomaly in the relative observation of $\nu_{\mu}$ and $\nu_{e}$ flux from cosmic rays striking the atmosphere was observed. Mainly produced in the atmosphere by the decay of pions $\pi^{+} \rightarrow \mu^{+}+\nu_{\mu} \rightarrow e^{+}+\nu_{e}+\overline{\nu_{\mu}}+\nu_{\mu}$ you would expect a relative production of 2 muon neutrinos for every electron neutrino. However first Kamiokande [38] and later IMB [39] (both water cherenkov detectors) detected a significant deficit of $\nu_{\mu}$ neutrinos but approximately the correct number of $\nu_{e}$ neutrinos. The measurement had some uncertainty because of additional neutrino production from Kaon decay but nothing close to the size of the discrepancy. In analogy to the solar neutrino anomaly this became known as the "atmospheric neutrino anomaly". 
The atmospheric neutrino anomaly was likewise shown to fit the predictions of the theory of neutrino oscillations by the next generation of experiments. A direct successor to Kamiokane, Super-Kamiokande, was built in 1996 and consisted of 50 kton of pure water surrounded by 11,000 photomultiplier tubes. By 1998 Super-K had observed the same up-down asymmetry in their observed $\nu_{\mu}$ deficit as Kamiokande, that is no deficit in down going muon events (which had traveled only the short distance from their creation in the atmosphere) and a significant deficit in up going events (which had traversed the diameter of the earth) [40].

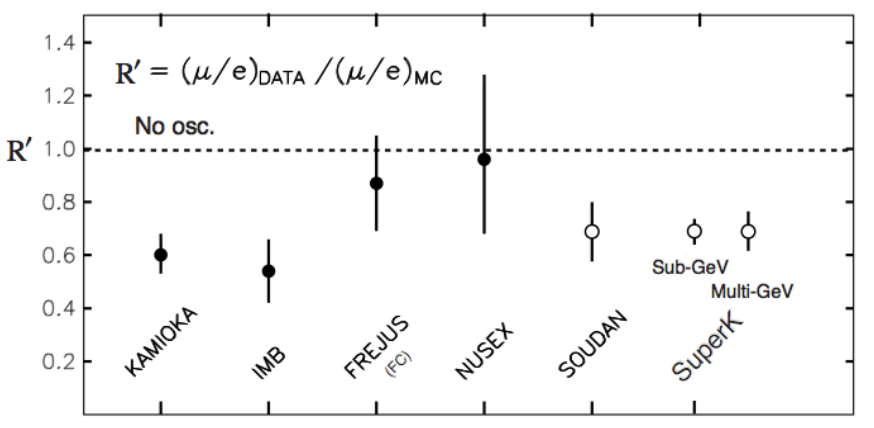

Figure 2.6: A summary of the deficit of $\frac{(\mu / e)_{D A T A}}{(\mu / e)_{M C}}$ as observed in a variety of experiments. Whilst FREJUS and NUSEX are both consistent, within uncertainties, with $R^{\prime}=1$ they are also consistent with $R^{\prime} \approx \frac{2}{3}$. Figure taken from [41].

\subsection{The Theory of Neutrino Oscillations}

The neutrino mass had not been measured to differ from zero, and it was defined to be zero by GSW. However it had been suggested by Pontecorvo as early as 1957 [42 that if the neutrino mass were non zero $\nu \rightarrow \bar{\nu}$ oscillations might occur (analogous to the Kaon system), in 1958 in light of new evidence for distinct lepton flavors that transitions between flavor states might be possible [43], and in 1962 the framework for neutrino oscillations as we know them now was laid down by Maki, Nakagawa, and Sakata as part of a separate effort in hadronic 
physics 44.

It is this theory of neutrino "oscillations" which has proven to give the best explanation of both the solar and atmospheric anomalies. The core idea is that any neutrino of a given lepton flavor $(\alpha)$ is produced with a superposition of different neutrino mass eigenstates (i):

$$
\left|\nu_{\alpha}\right\rangle=\sum_{i} U_{\alpha i}^{*}\left|\nu_{i}\right\rangle
$$

where the matrix element $U_{\alpha i}^{*}$ gives the relative amplitude for a given mass-flavor binding and the entire matrix is unitary such that $U U^{\dagger}=I$.

These masses propagate at different speeds and hence become out of phase as the neutrino travels such that the mass eigenstates evolve with time:

$$
\left|\nu_{\alpha}(t)\right\rangle=\sum_{i} U_{\alpha i}^{*} e^{i p_{i} \cdot x}\left|\nu_{i}\right\rangle
$$

where $\mathrm{x}$ is the four position of the neutrino and $p_{i}$ the four-momentum of the state i.

When the neutrino interacts at some time $t$ each mass state will have an associated superposition of different lepton flavors, and as the relative phases will have changed, it is entirely possible that the detected flavor will be different to the initial flavor state. The probability of this weak eigenstate $\mathrm{j}$ is found by summing over the mass eigenstates $\mathrm{j}$ :

$$
\left\langle\nu_{\beta}\right|=\sum_{j} U_{\beta j}\left\langle\nu_{j}\right|
$$

The probability of a neutrino of flavor $\alpha$ being flavor $\beta$ at time $\mathrm{t}$ is then given by the square of:

$$
\left\langle\nu_{\beta} \mid \nu_{\alpha}(t)\right\rangle=\sum_{j} \sum_{i} U_{\beta j} U_{\alpha i}^{*} e^{i p_{i} \cdot x}\left\langle\nu_{j} \mid \nu_{i}\right\rangle=\sum_{i} U_{\beta i} U_{\alpha i}^{*} e^{i p_{i} \cdot x}
$$


If we then call the $i^{\text {th }}$ neutrino energy and mass eigenstates $E_{i}$ and $m_{i}$, whilst assuming they all have the same squared three-momentum p:

$$
p_{i} \cdot x=E_{i} t-\mathbf{p} \cdot \mathbf{x}=\sqrt{|p|^{2}+m_{i}^{2}} t-\mathbf{p} \cdot \mathbf{x}
$$

Making the assumption that $m_{i}<<E_{i}$ and hence as a highly relativistic particles, $t=L$ and $\mathbf{p} \cdot \mathbf{x}=|\mathbf{p}| L$ (where $\mathrm{L}$ is the distance traveled) and $|\mathbf{p}| \approx E$ (as long as $m_{i}<<E_{i}$ and where $\mathrm{E}$ is the average over $E_{i}$ ):

$$
p_{i} \cdot x=|\mathbf{p}| L\left(1+\frac{m_{i}^{2}}{2|\mathbf{p}|^{2}}\right)-|\mathbf{p}| L=\frac{m_{i} L}{2 E}
$$

Which gives us:

$$
\left\langle\nu_{\beta} \mid \nu_{\alpha}(t)\right\rangle=\sum_{i} U_{\beta i} U_{\alpha i}^{*} e^{i \frac{m_{i} L}{2 E}}
$$

Such that we can now expand $P\left(\nu_{\beta} \rightarrow \nu_{\alpha}\right)$ as a function of L:

$$
\begin{gathered}
P\left(\nu_{\beta} \rightarrow \nu_{\alpha}\right)=\left|\left\langle\nu_{\beta} \mid \nu_{\alpha}(L)\right\rangle\right| \\
=\left(\sum_{j} U_{\beta j}^{*} U_{\alpha j} e^{-i \frac{m_{j} L}{2 E}}\right)\left(\sum_{i} U_{\beta i} U_{\alpha i}^{*} e^{i \frac{m_{i} L}{2 E}}\right) \\
=\sum_{i} \sum_{j} U_{\beta j}^{*} U_{\beta i} U_{\alpha i}^{*} U_{\alpha j} e^{-i \frac{\Delta m_{i j} L}{2 E}} \\
+\left[\sum_{i} \sum_{j} U_{\beta j}^{*} U_{\beta i} U_{\alpha i}^{*} U_{\alpha j}-\sum_{i} \sum_{j} U_{\beta j}^{*} U_{\beta i} U_{\alpha i}^{*} U_{\alpha j}\right]
\end{gathered}
$$

where $\Delta m_{i j}^{2}=m_{i}^{2}-m_{j}^{2}$ and the last two terms, which sum to zero, have been added to allow the following:

$$
P\left(\nu_{\beta} \rightarrow \nu_{\alpha}\right)=\sum_{i} \sum_{j} \underbrace{U_{\beta j}^{*} U_{\beta i} U_{\alpha i}^{*} U_{\alpha j}\left(e^{-i \frac{\Delta m_{i j} L}{2 E}}-1\right)}_{B_{i j}}+\underbrace{\sum_{i} \sum_{j} U_{\beta j}^{*} U_{\beta i} U_{\alpha i}^{*} U_{\alpha j}}_{A}
$$

If we now look at those individual terms, starting with A:

$$
A=\sum_{i} U_{\beta i} U_{\alpha i}^{*} \sum_{j} U_{\beta j}^{*} U_{\alpha j}
$$




$$
\begin{gathered}
=\sum_{i} U_{\beta i} U_{i \alpha}^{\dagger} \sum_{j} U_{j \beta}^{\dagger} U_{\alpha j} \\
=\delta_{\alpha \beta}
\end{gathered}
$$

where the unitary of $\mathrm{U}$ is used to arrive at $\delta_{\alpha \beta}$. If we now look at $B_{i j}$ we can see that $B_{i j}=B_{j i}$ and $B_{i i}=0$ and hence $B_{i j}+B_{j i}=2 \Re\left[B_{i j}\right]$. This allows us to remove those components of $B_{i j}$ where $i=j$ :

$$
P\left(\nu_{\beta} \rightarrow \nu_{\alpha}\right)=\delta_{\alpha \beta}+2 \sum_{i(>j)} \sum_{j} \Re\left[U_{\beta j}^{*} U_{\beta i} U_{\alpha i}^{*} U_{\alpha j}\left(e^{-i \frac{\Delta m_{i j} L}{2 E}}-1\right)\right]
$$

Finally using:

$$
e^{-i \frac{\Delta m_{i j} L}{2 E}}=\cos \left(\frac{\Delta m_{i j} L}{2 E}\right)+i \sin \left(\frac{\Delta m_{i j} L}{2 E}\right)
$$

and:

$$
\cos \left(\frac{\Delta m_{i j} L}{2 E}\right)-1=2 \sin \left(\frac{\Delta m_{i j} L}{2 E}\right)
$$

gives a survival probability equation of:

$$
\begin{gathered}
P\left(\nu_{\alpha} \rightarrow \nu_{\beta}\right)=\delta_{\alpha \beta} \\
+2 \sum_{i(>j)} \sum_{j} \Im\left[U_{\beta j}^{*} U_{\beta i} U_{\alpha i}^{*} U_{\alpha j}\right] \sin \left(\frac{\Delta m_{i j}^{2} L}{2 E}\right) \\
-4 \sum_{i(>j)} \sum_{j} \Re\left[U_{\beta j}^{*} U_{\beta i} U_{\alpha i}^{*} U_{\alpha j}\right] \sin ^{2}\left(\frac{\Delta m_{i j}^{2} L}{4 E}\right)
\end{gathered}
$$

\subsubsection{Three flavor oscillations}

To describe the theory of neutrino oscillations we normally assume three lepton and mass eigenstates, which is reasonable in the light of the LEP data. A particular formalism of $\mathrm{U}$ is used, often referred to as the PMNS rotation matrix. Recalling that $U^{\dagger} U=I$, the unitary constraint removes 9 free parameters, 5 are just relative phases between the lepton fields, and can be absorbed into those same fields. The remaining four free parameters in $\mathrm{U}$ are chosen such that the 
parametrization is in terms of three mixing angles $\theta_{12}, \theta_{23}, \theta_{13}$ and a phase $\delta$ :

$$
\begin{aligned}
& \left(\begin{array}{c}
\nu_{e} \\
\nu_{\mu} \\
\nu_{\tau}
\end{array}\right)=U^{*}\left(\begin{array}{l}
\nu_{1} \\
\nu_{2} \\
\nu_{3}
\end{array}\right) \\
& U=\left(\begin{array}{ccc}
U_{e 1} & U_{e 2} & U_{e 3} \\
U_{\mu 1} & U_{\mu 2} & U_{\mu 3} \\
U_{\tau 1} & U_{\tau 2} & U_{\tau 3}
\end{array}\right) \\
& U=\underbrace{\left(\begin{array}{ccc}
1 & 0 & 0 \\
0 & \cos \left(\theta_{23}\right) & \sin \left(\theta_{23}\right) \\
0 & -\sin \left(\theta_{23}\right) & \cos \left(\theta_{23}\right)
\end{array}\right)}_{\text {Atmospheric }} \underbrace{\left(\begin{array}{ccc}
\cos \left(\theta_{13}\right) & 0 & \sin \left(\theta_{13}\right) e^{-i \delta} \\
0 & 1 & 0 \\
-\sin \left(\theta_{13}\right) e^{-i \delta} & 0 & \cos \left(\theta_{13}\right)
\end{array}\right)}_{\text {Cross Mixing }} \underbrace{\left(\begin{array}{ccc}
\cos \left(\theta_{12}\right) & \sin \left(\theta_{12}\right) & 0 \\
-\sin \left(\theta_{12}\right) & \cos \left(\theta_{12}\right) & 0 \\
0 & 0 & 1
\end{array}\right)}_{\text {Solar }} \\
& U=\left(\begin{array}{ccc}
c_{12} c_{13} & s_{12} c_{13} & s_{13} e^{-i \delta} \\
-s_{12} c_{23}-c_{12} s_{23} s_{13} e^{i \delta} & c_{12} c_{23}-s_{12} s_{23} s_{13} e^{i \delta} & s_{23} c_{13} \\
s_{12} s_{23}-c_{12} c_{23} s_{13} e^{i \delta} & -c_{12} s_{23}-s_{12} c_{23} s_{13} e^{i \delta} & c_{23} c_{13}
\end{array}\right)
\end{aligned}
$$

where $s_{i j}=\sin \left(\theta_{i j}\right)$ and $c_{i j}=\cos \left(\theta_{i j}\right)$.

\subsubsection{The Two Flavor Approximation}

It can be shown that the three flavor equation can be folded down to a two flavor approximation for most experiments. This is demonstrated below for the MINOS experiment.

First we move to SI units, such that the last argument of the last sinusoidal term in the three flavor survival probability equation above becomes:

$$
\frac{\Delta m_{i j}^{2} c^{4} L}{4 E \hbar c}
$$

and if we leave $\mathrm{E}$ in units of $\mathrm{GeV}, \mathrm{L}$ in $\mathrm{km}$ and $\Delta m_{i j}^{2}$ in $e V^{2}$, setting $\hbar=$ $6.58 \times 10^{-25} \mathrm{GeVs}, c=3 \times 10^{5} \mathrm{kms}^{-1}, \Delta m_{i j}^{2} \mathrm{c}^{4}=\Delta m_{i j}^{2} \times 10^{-18}\left(\mathrm{eV}^{2} / \mathrm{GeV}^{2}\right)$, we 
get:

$$
\frac{1.27 \Delta m_{i j}^{2} L}{E}
$$

Now the transition probability relevant to MINOS is that of muon disappearance so we can say $\alpha=\beta=\mu$ and hence the imaginary term in the three flavor survival probability equation goes to zero and we are left with:

$$
P\left(\nu_{\mu} \rightarrow \nu_{\mu}\right)=1-4 \sum_{i(>j)} \sum_{j}\left|U_{\mu i}\right|^{2}\left|U_{\mu j}\right|^{2} \sin ^{2}\left(\frac{1.27 \Delta m_{i j}^{2} L}{E}\right)
$$

We know from several leading neutrino experiments, which we will discuss later in this chapter, that $\sin \left(\theta_{13}\right)$ is very small, and so if we approximate $\sin \left(\theta_{13}\right)=0$ and $\cos \left(\theta_{13}\right)=1$ the relevant PMNS matrix components become:

$$
\begin{gathered}
U_{\mu 1}=s_{12} c_{23}-c_{12} s_{23} s_{13} e^{i \delta} \Rightarrow\left|U_{\mu 1}\right|^{2} \approx s_{12}^{2} c_{23}^{2} \\
U_{\mu 2}=-c_{12} c_{23}-s_{12} s_{23} s_{13} e^{i \delta} \Rightarrow\left|U_{\mu 2}\right|^{2} \approx c_{12}^{2} c_{23}^{2} \\
U_{\mu 3}=s_{23} c_{13} \Rightarrow\left|U_{\mu 3}\right|^{2} \approx s_{23}^{2}
\end{gathered}
$$

Furthermore experiments have shown, as we will discuss later in this chapter, that $\Delta m_{21}$ is of order $7.6 \times 10^{-5} \mathrm{eV}^{2}$. Therefore for MINOS we also have $L=735 \mathrm{~km}$ and $E=3 G e V$ :

$$
\sin ^{2}\left(\frac{1.27 \Delta m_{21}^{2} L}{E}\right)=\sin ^{2}\left(\frac{1.27 \times 7.6 \times 10^{-5} \times 735}{3}\right) \approx \sin ^{2}(0.02) \approx 0
$$

and then using that $\Delta m_{31}^{2}$ is of order $2.4 \times 10^{-3} \mathrm{eV}^{2}$ and hence $\left(\gg \Delta m_{21}^{2}\right)$, and also that $\Delta m_{31}^{2}-\Delta m_{21}^{2}=\Delta m_{32}^{2}$ we can say that $\Delta m_{31}^{2} \approx \Delta m_{32}^{2}=\Delta m_{a t m}^{2}$. Giving:

$$
P\left(\nu_{\mu} \rightarrow \nu_{\mu}\right)=1-4 s_{23}^{2} c_{23}^{2}\left(s_{12}^{2}+c_{12}^{2}\right) \sin ^{2}\left(\frac{1.27 \Delta m_{a t m}^{2} L}{E}\right)
$$

and then using $2 \sin \theta \cos \theta=\sin ^{2} \theta$ and that $\sin ^{2} \theta+\cos ^{2} \theta=1$ brings us to the two flavour approximation for muon neutrino survival at MINOS:

$$
P\left(\nu_{\mu} \rightarrow \nu_{\mu}\right) \approx 1-\sin ^{2}\left(2 \theta_{23}\right) \sin ^{2}\left(\frac{1.27 \Delta m_{a t m}^{2} L}{E}\right)
$$




\subsubsection{Matter Effects}

Whilst the interaction of neutrinos with matter is famously weak, neutrinos traveling through matter do undergo a modification of their oscillatory behavior as a result. This is most pronounced in neutrinos which have passed through long distances or high densities of matter, such as neutrinos from nuclear interactions in the center of the sun. These modifications are known as the Mikheev-SmirnovWolfenstein or MSW effect [45] [46].

The effect itself comes from coherent forward scattering with the protons, neutrons, and electrons which make up every day matter. Every (non sterile) neutrino will therefore undergo NC scattering with an interaction potential:

$$
V_{m}^{N C}=\frac{\mp 1}{\sqrt{2}} G_{F} n_{n}
$$

where $n_{n}$ is the number density of neutrons, $G_{F}$ is the Fermi constant, and the lower of the $\mp$ signs corresponds to the anti-neutrino potential. All neutrinos experience this potential equally, so it will appear in the Hamiltonian as a multiple of the identity matrix. As all neutrinos experience this potential the same way it has no physically observable effect on the oscillation probabilities. More interesting is the additional $\mathrm{CC}$ interaction which electron and anti electron neutrinos will experience with the electrons in normal matter representable as:

$$
V_{m}^{C C}= \pm \sqrt{2} G_{F} n_{e}
$$

where $n_{e}$ is the number density of electrons. Affecting only one flavor of neutrino this potential can change the relative oscillation rate. In some exotic tauon/muon rich sample of matter the effect would be similarly skewed relative to tau or muon neutrinos.

If we consider a simple two flavor oscillation scenario we can show how this effect 
would appear. Starting with the $2 \times 2$ version of the PMNS matrix $U_{2}$, defined as:

$$
U_{2}(\theta)=\left(\begin{array}{cc}
\cos \theta & \sin \theta \\
-\sin \theta & \cos \theta
\end{array}\right)
$$

where:

$$
\left(\begin{array}{l}
\nu_{e} \\
\nu_{\beta}
\end{array}\right)=U\left(\begin{array}{l}
\nu_{1} \\
\nu_{2}
\end{array}\right)
$$

And our hamiltonian defined as:

$$
H=U_{2}(\theta) H_{v a c} U_{2}^{\dagger}(\theta)+H_{c c}
$$

Where:

$$
\begin{gathered}
H_{v a c}=\left(\begin{array}{cc}
\frac{\Delta m^{2}}{2 E} & 0 \\
0 & \frac{\Delta m^{2}}{2 E}
\end{array}\right) \\
H_{c c}=\left(\begin{array}{cc}
V_{m}^{e} & 0 \\
0 & 0
\end{array}\right)
\end{gathered}
$$

Such that:

$$
\begin{aligned}
H & =U_{2}(\theta)\left(\begin{array}{cc}
\frac{\Delta m^{2}}{2 E} & 0 \\
0 & \frac{\Delta m^{2}}{2 E}
\end{array}\right) U_{2}^{\dagger}(\theta)+\left(\begin{array}{cc}
V_{m}^{e} & 0 \\
0 & 0
\end{array}\right) \\
& =\frac{\Delta m^{2}}{2 E}\left(\begin{array}{cc}
\sin ^{2} \theta+\frac{2 E}{\Delta m^{2}} V_{m}^{e} & -\sin \theta \cos \theta \\
-\sin \theta \cos \theta & \cos ^{2} \theta
\end{array}\right)
\end{aligned}
$$

If we define $A_{2}=\frac{2 E}{\Delta m^{2}} V_{m}^{e}$ we can diagonalize this equation by creating a new mixing angle $\theta_{m}$ and energy eigenvalues $E_{1,2}^{m}$ such that:

$$
\begin{gathered}
C_{2}=\sqrt{\sin ^{2} 2 \theta+\left(\cos 2 \theta-A_{2}\right)^{2}} \\
\cos 2 \theta_{m}=\frac{\cos 2 \theta-A_{2}}{C_{2}} \\
\sin 2 \theta_{m}=\frac{\sin 2 \theta}{C_{2}} \\
E_{1,2}^{m}= \pm \frac{\Delta m^{2}}{4 E} C_{2}
\end{gathered}
$$


We can then solve this equation as in the two flavor approximation, but now the mixing angle and mass spacing have both been modified such that:

$$
P\left(\nu_{e} \rightarrow \nu_{e}\right)=1-\frac{\sin ^{2}(2 \theta)}{C_{2}^{2}} \sin ^{2}\left(\frac{\Delta m^{2} C_{2} L}{4 E}\right)
$$

From these modifications we can see that matter effects can shift both the frequency and amplitude of oscillations. Note also that neutrino oscillations in matter can be maximal regardless of the mixing angle. When:

$$
n_{e}=\frac{\Delta m^{2} \cos 2 \theta}{2 \sqrt{2} G_{F} E}
$$

$\sin ^{2} 2 \theta^{m}=1$ no matter the value of $\theta$. Perhaps most interestingly when mutliplied out the term $\Delta m^{2} C_{2}$ will have a term of the form $\left(\Delta m^{2} \cos 2 \theta-2 E V_{m}^{e}\right)^{2}$ such that matter effects add or subtract from $\Delta m^{2}$ directly, potentially allowing you to resolve the mass hiearchy.

\subsection{Measurement of the Neutrino Oscillation Mix- ing Parameters so Far}

Any neutrino oscillation probability can be described in terms of $\Delta m_{21}, \Delta m_{31}$, $\Delta m_{32}, \theta_{12}, \theta_{23}, \theta_{13}$ and a phase $\delta_{c p}$. Certain combinations of $\mathrm{L} / \mathrm{E}$ are sensitive to particular combinations of these mixing parameters, and the three that the field has explored are refered to as Solar, Atmospheric and Reactor oscillations respectively. They are named for the first experiments to measure neutrino oscillations in that $\mathrm{L} / \mathrm{E}$ range.

\subsubsection{Solar Neutrino Oscillations}

At the Solar L/E electron neutrino survival probability is dominated by $\Delta m_{21}$ and $\sin ^{2} \theta_{12}$, and it is from SNO and Kamland that we get our leading measure 
of these mixing paramaters.

Interpretation of the SNO result is somewhat complicated by the additional consideration of matter effects in the sun. Oscillations in the vacuum between the sun and the earth are heavily disfavored as there is no observed seasonal variation in the solar neutrino flux, beyond a $\frac{1}{r^{2}}$ as expected as the vacuum part of the baseline varies due to the Earth's mildly eccentric orbit. Similarly the absence of any day-night variations from matter effects in the earth rules out a region around $\Delta m_{21}^{2} \cos 2 \theta_{12} \approx 10^{-6} \mathrm{eV}^{2}$.

Instead, the data favors a large mixing angle (LMA) solution where matter effects are driven by adiabatic passage through the MSW resonance within the sun. In this solution the density at the center of the sun is far above the critical density where $N_{e} \gg N_{e}^{r e s}$ and hence $\theta_{M}$ is almost $90^{\circ}$. The density in the sun varies approximately smoothly with its radius so the resonance is passed adiabatically and the neutrinos stay in their $\nu_{2}$ state such that at the point when the solar $\nu_{e}$ leave the sun they are almost entirely $\nu_{2}$.

Once they exit the sun and into the vacuum the state will then be a mixture of electron and other flavors such that $\left|\nu_{2}\right\rangle=\sin \theta_{12}\left|\nu_{e}\right\rangle+\cos \theta_{12}\left|\nu_{x}\right\rangle$ and the detected fraction of the expected $\nu_{e}$ is then $\sin ^{2} \theta_{12}$. Constraints on $\Delta m_{21}^{2}$ are far looser than on $\sin ^{2} \theta_{12}$ and only require that the resonance happen gradually at some point between the core and the surface of the sun. This interpretation means that SNO has measured $\sin ^{2} \theta_{21} \approx \frac{1}{3}$. This solution is only valid for $\operatorname{sgn}\left(\Delta m_{21}^{2}\right)=+1$ as both the sun and neutrinos are matter and $\cos \theta_{12} \approx \frac{1}{3}$ (where convention dictates that the heavier mass state is labeled $\nu_{2}$ and the lighter $\left.\nu_{1}\right)$. 
The neutrino experiment KamLAND also explored the solar L/E. KamLAND used a liquid scintillator detector to observe electron anti neutrinos neutrino from Japan's (at the time) 53 local nuclear power stations [47]. With an average baseline of $180 \mathrm{~km}$ and detector energy threshold of $1.8 \mathrm{MeV}$ KamLAND was sensitive to oscillations as described by the LMA mass splitting detailed above. Not only was KamLAND able to verify the solar parameters as measured by SNO, it was able to observe in detail the shape of the dip in the expected spectrum caused by oscillations. It provided a much higher precision measurement of the mass splitting than SNO (and other similar solar experiments) which see only a rate at a very narrow range of $\mathrm{L} / \mathrm{E}$. This ratio of expected to observed spectra can be seen in Figure 2.7. The comparison of the solar and reactor best fits to the solar mixing parameters is shown in figure 2.8

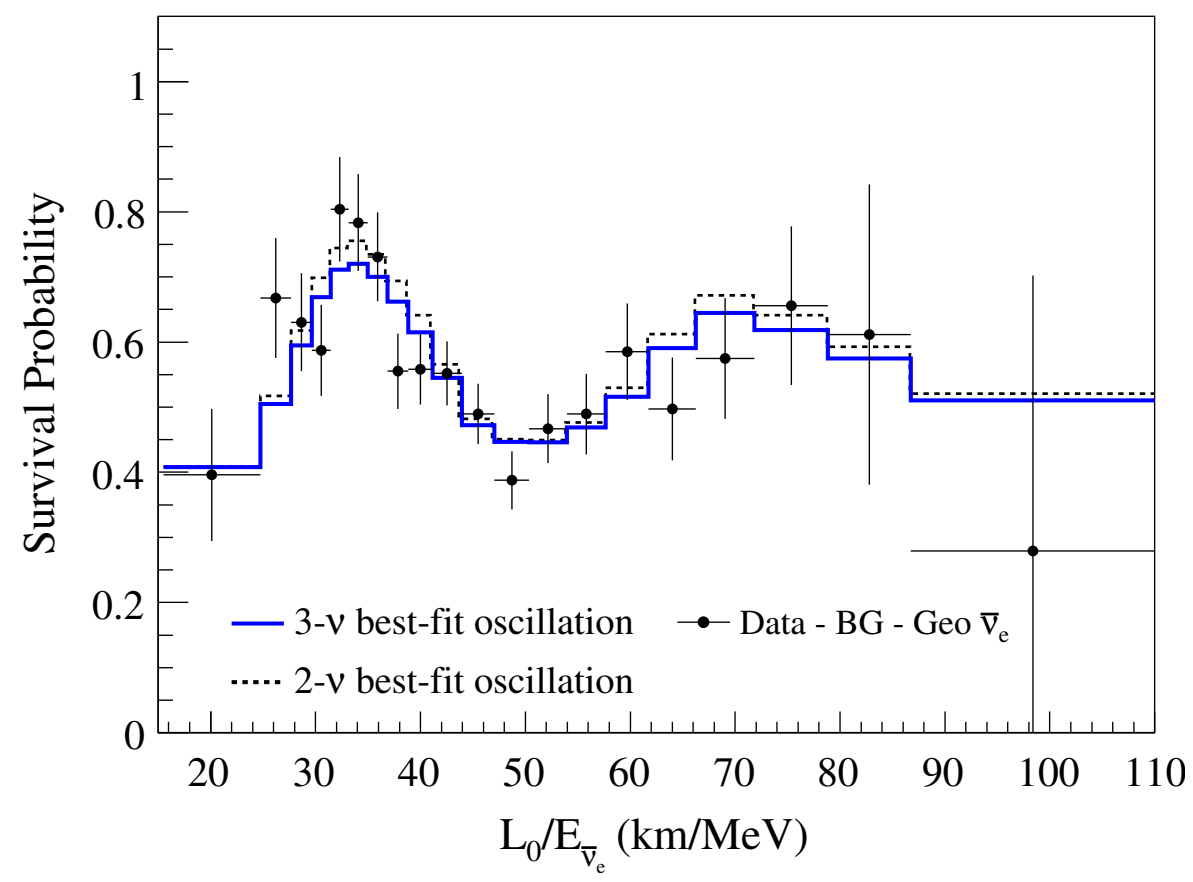

Figure 2.7: Shown in black the ratio of measured to expected $\bar{\nu}_{e}$ as a function of distance traveled by the neutrino divided by its energy. The blue line shows the best fit for the oscillation hypothesis. Figure taken from [47] 


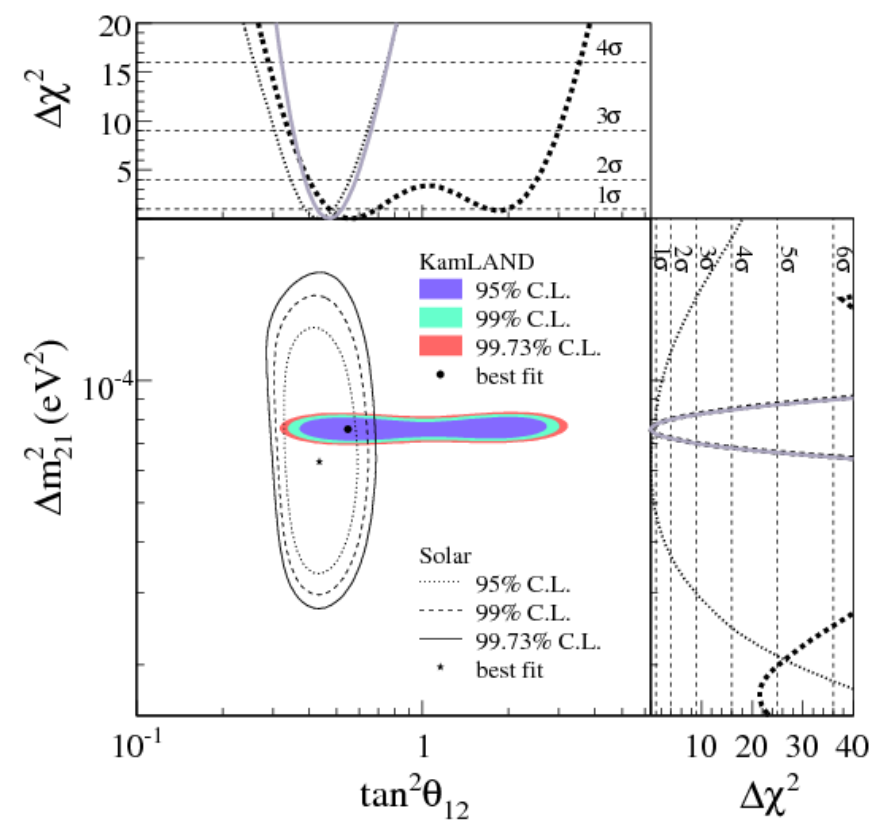

Figure 2.8: Measurements of the solar mixing parameters from SNO and other radio chemical experiments (black) and KamLAND (colored region). Not only are the contours in good agreement, they also provide complementary information. Figure taken from [47]

Taken in combination the worlds best knowledge of the solar mixing parameters 48 is:

$$
\begin{gathered}
\Delta m_{21}^{2}=7.54_{-0.22}^{+0.26} \times 10^{-5} \mathrm{eV}^{2} \\
\sin ^{2} \theta_{12}=0.307_{-0.016}^{+0.018}
\end{gathered}
$$

\subsubsection{Atmospheric Neutrino Oscillations}

At the Atmospheric L/E muon neutrino survival probability is dominated by $\Delta m_{a t m}^{2}$ and $\sin ^{2} \theta_{23}$, and it is from Super-K and MINOS that we get our leading measure of these mixing parameters. $\Delta m_{\text {atm }}^{2}$ is a combination of $\Delta m_{31}$ and $\Delta m_{32}$ derived in section 2.5.2 of this thesis. 
When interpreted as the result of neutrino oscillations these observations of muon neutrino disappearance over a $500 \mathrm{~km} / \mathrm{Gev} \mathrm{L} / \mathrm{E}$ allow strong limits to be placed on the parameters governing atmospheric neutrino oscillations. The analysis is performed twice to provide maximally accurate measurments of both $\theta_{23}$ and $\Delta\left|m_{\text {atm }}^{2}\right|$. Normally the data is binned in terms of zenith angle, as shown in Figure 2.9 such that there are really only two baselines or L/E's observed and hence sensitivity to the atmospheric mass splitting is limited. A more precise measurement of the mass splitting is possible with the same dataset if you first select events whose L/E can be well estimated and then use the oscillation dip observable in your plot of events vs. L/E, as shown in Figure 2.10, to fit to the atmospheric mixing parameters though the measurement of the mixing angle then becomes far less precise. 

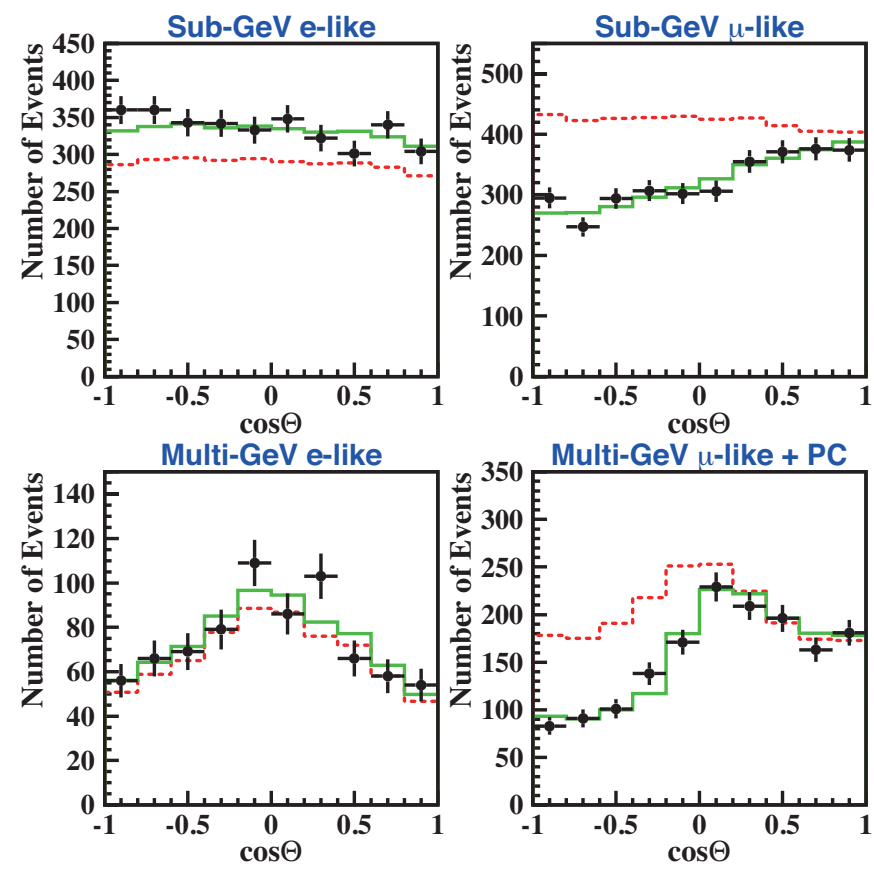

Figure 2.9: Atmospheric neutrino event rates measures by Super Kamiokande as a function of zenith angle. Shown are $\nu_{e}$ events (left column), $\nu_{\mu}$ events (right column), high energy events (bottom row) and low energy events (top row). Data (black dots), prediction with null oscillation (red) and prediction with the best fit to oscillations (green) is shown for each set of events. Figure taken from [49] 


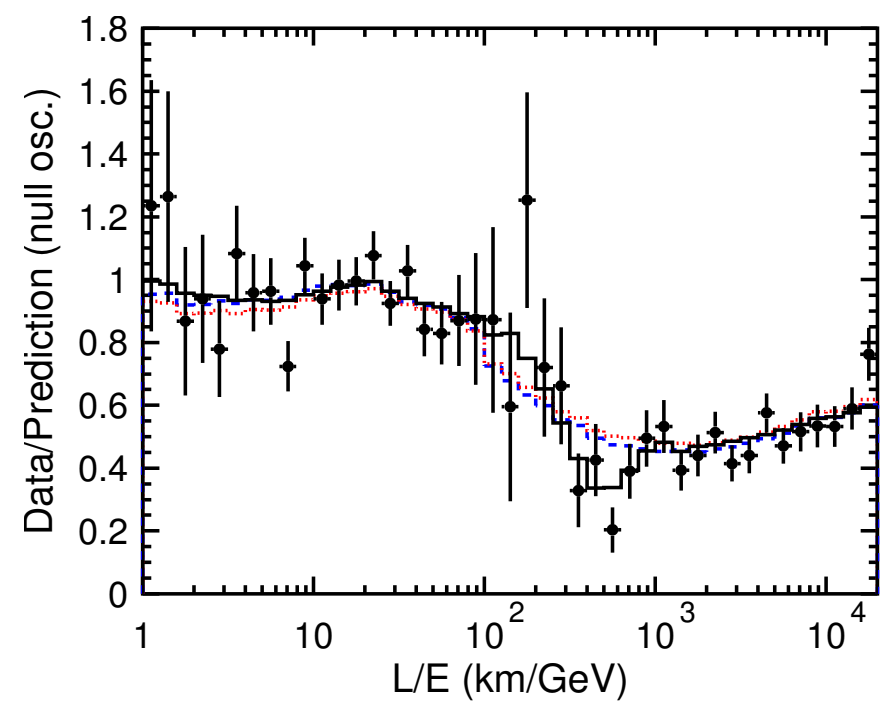

Figure 2.10: The ratio of measured atmospheric neutrino events rates to a null oscillation case as a function of the distance traveled divided by the energy of the neutrino. Show is the data (black points), the best fit to oscillation (black line), the best fit to decoherance (red line) and neutrino decay (blue line). Figure taken from [40]

In analogy to KamLAND a number of experiments have measured atmospheric mixing with careful observation of an artificial source of muon neutrinos. Accelerator driven protons impinge on carbon targets to produce pions which will decay into a beam of muon neutrinos. KEK to Kamiokande or K2K was the first such experiment, using a beam of muon neutrinos produced at KEK from a 12 $\mathrm{GeV}$ proton beam on an aluminium target. The beam was measured both at a 1kton water Cerenkov detector just 300m downstream of the target and again at Super Kamiokande $250 \mathrm{~km}$ away. This allowed the beam properties to be known before and after oscillations. With the advantage of a known length $\mathrm{L}$ they could fit over bins of E to get a result consistent with the Super Kamiokande best fit.

The MINOS experiment, which will be covered in detail in the remaining chapters 
of this thesis, follows very similar principles to K2K. It studies a beam of muon neutrinos, peaked at $3 \mathrm{GeV}$, first at 1000m and then again at a $735 \mathrm{~km}$ baseline with a pair of almost identical detectors which are capable of highly accurate reconstruction of the energy of the interacting muon neutrino. By looking at the position and depth of the disappearance of neutrinos in the beam as observed and as expected in the case of null oscillations the experiment has produced the leading measurement of the atmospheric mass splitting and the details of this analysis will also be covered in latter chapters of this thesis. The Super Kamiokande [50] best fit to $\sin ^{2} 2 \theta_{23}$ and the MINOS[51] best fit to $\Delta m_{a t m}^{2}$ are:

$$
\begin{gathered}
\sin ^{2} 2 \theta_{23}>0.93(90 \% \text { C.L. }) \\
\left|\Delta m_{\text {atm }}^{2}\right|=2.41_{-0.10}^{+0.09} \times 10^{-3} \mathrm{eV}^{2}
\end{gathered}
$$

Figure 2.11 shows the worlds knowledge of the atmospheric mixing parameters. 


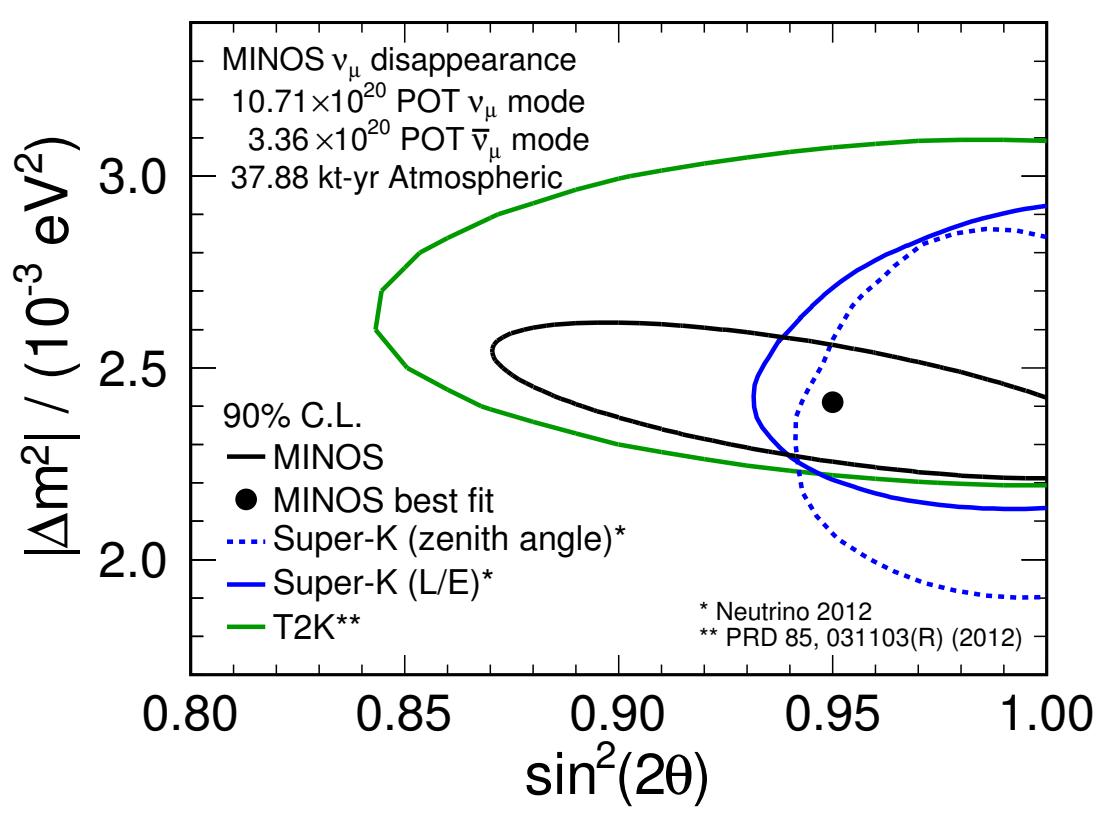

Figure 2.11: Confidence limits on the parameters $\left|\Delta m^{2}\right|$ and $\sin ^{2} 2 \theta$, assuming equal oscillations for neutrinos and antineutrinos. The black curve gives the $90 \%$ contour obtained from the combined analysis of MINOS accelerator and atmospheric neutrinos. For comparison, the blue curves show the $90 \%$ contours from Super-K (zenith angle analysis indicated by dotted line, L/E analysis indicated by solid line) and the green curve shows the $90 \%$ contour from T2K. Figure taken from [51]

\subsubsection{Reactor Neutrino Oscillations}

At the Reactor L/E electron neutrino survival probability is dominated by $\Delta m_{\text {atm }}^{2}$ and $\sin ^{2} \theta_{13}$, and it is from Daya Bay that we get our leading measure of $\sin ^{2} \theta_{13}$. $\Delta m_{a t m}^{2}$ is best measured by atmospheric oscillations experiments such as MINOS and both Daya Bay and other reactor experiments have historically used the MINOS best fit to $\Delta m_{a t m}^{2}$ as a fixed parameter in their fits. Within its uncertainties $\Delta m_{a t m}^{2}$ has very little effect on their result [52]. 
Attempts to measure $\theta_{13}$ have historically taken two forms. One being the search for the appearance of electron neutrinos in beams of muon neutrinos with experiments such as MINOS and more recently Tokei to Kamiokande (T2K). The other being the study of the disappearance of electron anti-neutrinos from nuclear reactors by experiments such as Daya Bay, in a method analogous to KamLAND, albeit at much shorter baselines where the solar mixing angle doesn't contribute.

Ultimately it is from the reactor experiment Daya Bay that we have very recently received a very precise measurement of $\theta_{13}$ [52]. The experiment consists of two near and one far sets of detectors which are 470/576 m and $1648 \mathrm{~m}$ away respectively from a set of 6 nuclear reactors which produce a high flux of $\approx 3$ $\mathrm{MeV}$ electron anti-neutrinos. Each detector module consists of three acrylic vessels nested inside one another, with a core of $20 \mathrm{t}$ of Gd-LS, a middle layer of $20 \mathrm{t}$ of liquid scintillator, and an outer layer of $37 \mathrm{t}$ of mineral oil shielding against background radiation. Each detector is essentially a more sophisticated version of the experiment Cowan conducted to detect the first neutrino and again $\overline{\nu_{e}}+p \rightarrow e^{+}+n$ with a prompt scintillation from the $e^{+}$and a delayed signal from the capture of the associated neutrino on Gd provided a distinctive signal. Comparing expected and observed event rates they were able to put strong limits on the reactor mixing angle. Their best fit is:

$$
\sin ^{2} \theta_{13}=0.089 \pm 0.011
$$




\subsection{Ongoing and Future Efforts}

\subsubsection{Majorana Neutrinos}

Within the Standard Model, neutrinos are Dirac particles, distinctly different to their anti-neutrino equivalents. However it has been suggested[53] that neutrinos could be Majorana particles, where both neutrino and anti-neutrino are the same particle. The right handed neutrino is then what we previously interpreted as the right handed anti-neutrino. Experiments, such as Cuore [54] search for a signature neutrinoless double beta decay shown in Figure 2.12. To date no concrete evidence has been found.

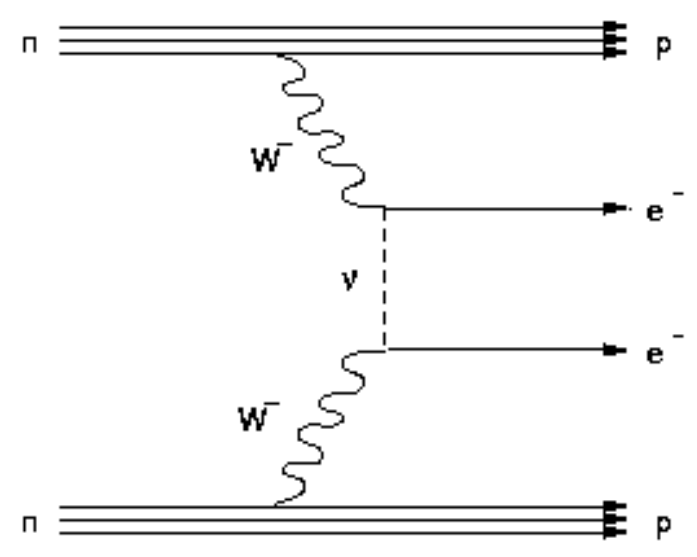

Figure 2.12: Feynman diagram showing neutrinoless double beta decay.

\subsubsection{The Resolution of the Mass Hierarchy}

The three mass eigenstates do not directly effect neutrino oscillations, rather it is the difference between the mass eigenstates which is measured in any study of neutrino oscillations: $\Delta m_{12}^{2}$ and $\left|\Delta m_{\text {atm }}^{2}\right|$ (a combination of $\Delta m_{31}^{2}$, and $\Delta m_{32}^{2}$ derived in section 2.5.2) have been measured to a high degree of accuracy, as discussed in the previous chapter. 
However their full hierarchy is still unknown. Deriving the sign of any mass splitting requires a significant contributions from matter effects on the oscillation probability, as shown in section 2.5.3, thus we can derive the sign of $\Delta m_{21}^{2}$ because matter effects in the sun have such a strong effect on the oscillation of neutrinos from the heart of the sun. However to date no terrestial atmospheric experiment has had a large enough contribution from matter effects to calculate the sign of $\left|\Delta m_{\text {atm }}^{2}\right|$ in combination with the energy resolution to have sensitivity to the size of $\left|\Delta m_{a t m}^{2}\right|$.

Furthermore our leading measure of $\left|\Delta m_{\text {atm }}^{2}\right|$ comes from accelerator experiments such as MINOS which until recently used a two flavour approximation of neutrino oscillations without matter effects, and therefore have precisely no sensitivity to the mass hiearchy. Chapter 6 of this thesis will describe the work towards a hiearchy sensitive measurement of the atmospheric mass splitting at MINOS.

Several planned future experiments will have the prerequisite baseline through matter. Accelerator driven experiments such as NOvA and LBNE will have longer baselines, lower energies, and higher intensities than any previous neutrino beam experiments. Their larger data sample and longer baselines will give them some sensitivity to the mass hierarchy. Additionaly the next generation of neutrino observatories such as the Super-K successor Hyper-K or the Icecube upgrade PINGU will use baselines on the order of the length of the earth in combination with better energy resolution and detector volumes to gain sensitivity to the mass hierarchy. 


\subsubsection{Measurement of $\delta_{c p}$}

The oscillation of neutrinos from one lepton flavor to another already represents beyond the Standard Model physics. With the measurement of a non zero $\theta_{13}$ by Daya Bay a non zero $\delta_{c p}$ would be the first example of CP violation in the Lepton sector and would have ramifications for the theory of leptongenesis [53].

It can be shown that matter effects can give you sensitivity to $\delta_{c p}$ and the leading method for a definitive measure of $\delta_{c p}$ is using an accelerator driven experiment and comparing oscillation probabilities for $P\left(\nu_{\mu} \rightarrow \nu_{e}\right)$ to $P\left(\bar{\nu}_{\mu} \rightarrow \bar{\nu}_{e}\right)$. Complicating this measurement is the fact that mass hierarchy and $\delta_{c p}$ affect oscillation probabilities in a similiar range of $\mathrm{L} / \mathrm{E}$ such that there is a degeneracy between measurement of the two.

\subsubsection{Sterile Neutrinos}

As discussed earlier there is strong evidence from measurements of $\mathrm{Z}$ decay that there are three lepton flavors and hence three neutrinos. However if a neutrino were to be sterile, that is lacking any lepton flavor, then the $\mathrm{Z}$ decay limit would not be applicable. Neutrino oscillation experiments can search for these sterile neutrinos by looking for evidence that the weakly charged neutrinos have oscillated into a sterile neutrino, the most distinctive signal being an energy dependent deficit in the rate of $\mathrm{NC}$ interactions as the sterile neutrino cannot couple to the $\mathrm{Z}$ boson. To date no experiment has found strong evidence for sterile neutrinos, although the short baseline neutrino beam experiments LSND [55] and Miniboone [56] have seen $\nu_{e}$ excesses which could be explained by changes to standard three flavor oscillations by the existence of one or more sterile neutrinos.

The MINOS experiment conducts its own Sterile neutrino search by looking for 
energy dependent NC event rate deficits anywhere in our observed NC spectrum and event rate deficits at high energies in our CC spectrum away from the atmospheric oscillation maximum. An update should be released very soon and the projected sensitivity is shown in Figure 2.13. At high $\Delta m_{41}^{2}$ our sensitivity suffers as oscillations to the sterile neutrino are too rapid to measure with a Near/Far comparison with the MINOS detectors, occurring before the Near Detector. At low $\Delta m_{41}^{2}$ our sensitivity again suffers as sterile oscillations occur too close to the atmospheric oscillation maximum to distinguish between the two. 


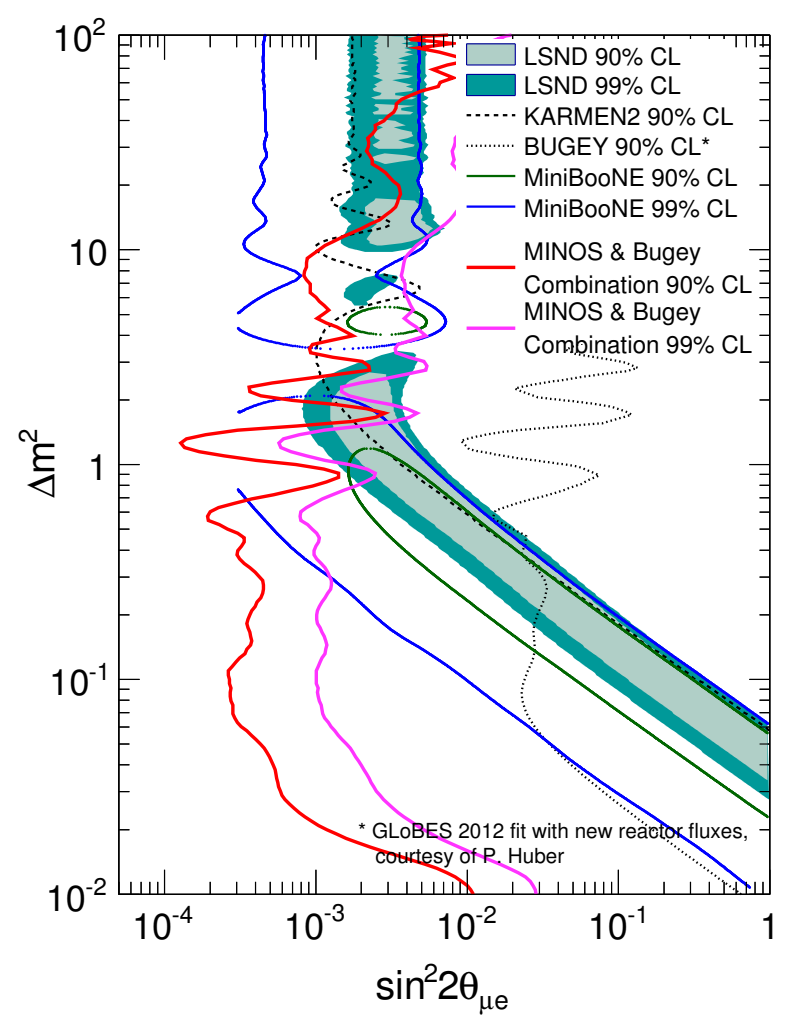

Figure 2.13: MINOS and Bugey combined 90\% and 99\% confidence level limits on the sterile mixing parameter $\sin ^{2} 2 \theta \mu e=4|U e 4|^{2}|U \mu 4|^{2}$, obtained from the individual disappearance limits of each experiment on the size of $|U \mu 4|^{2}$ and $|U e 4|^{2}$, respectively. Regions of parameter space to the right of the red contour are excluded at $90 \%$ CL, while those to the right of the magenta contour are excluded at $99 \%$ CL. The MINOS limits correspond to a $10.71 \times 10^{20}$ POT exposure in neutrino running mode. The Bugey limits are computed from a GLoBES 2012 fit provided by P. Huber. It accounts for the new calculation of reactor fluxes, as described in P. Huber, Phys. Rev. C 85029901 (2011). The MiniBooNE contours are provided by the MiniBooNE Collaboration and were published in A.A. Aguilar-Arevalo et al., Phys. Rev. Lett. 110, 161801 (2013) 


\section{Chapter 3}

\section{The MINOS Experiment}

MINOS or Main Injector Neutrino Oscillation Search is a long baseline neutrino oscillation experiment. The experiment uses two detectors placed at $1 \mathrm{~km}$ and $735 \mathrm{~km}$ downstream of this beam to measure the neutrino composition at each point, Figure 3.1 shows this baseline. The NuMI beam is composed of predominantly muon neutrinos made by impinging $120 \mathrm{GeV}$ protons onto a carbon target to produce mesons which decay into neutrinos.

The following sections will discuss the physics goals of this experiment and the elements of its design and operation which make it suited to those goals.

\subsection{Physics Goals}

The primary physics goal of the MINOS experiment is the measurement of the atmospheric neutrino mixing parameters through the observation of $\nu_{\mu}$ disappearance in the NuMI beam over its $735 \mathrm{~km}$ baseline. The $735 \mathrm{~km}$ baseline and the peak neutrino energy of $3 \mathrm{GeV}$ are chosen such that upon reaching the Far Detector the $\nu_{\mu}$ beam is at the peak of its first oscillation peak from $\nu_{\mu}$ to $\nu_{\tau}$. 

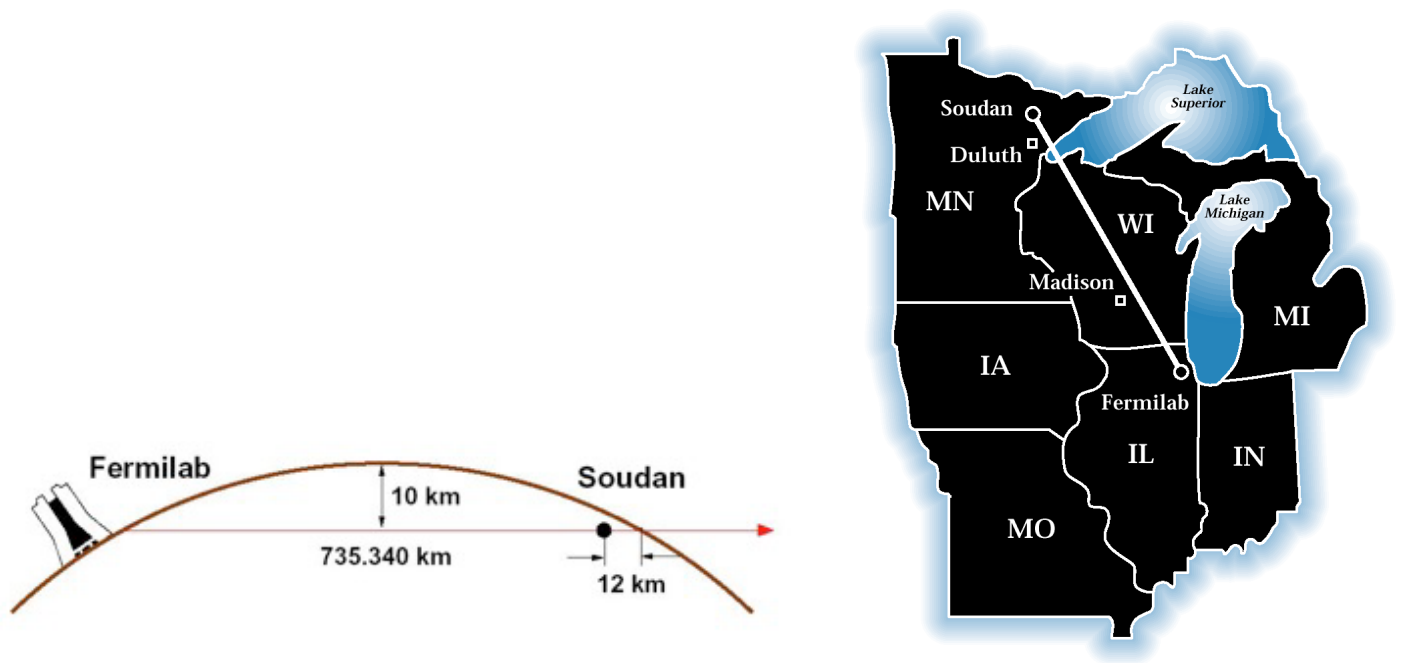

Figure 3.1: A sketch showing the baseline of the MINOS experiment and the location of our Near and Far Detectors withing the NuMI beam.

The Near Detector allows a precise measurement of the neutrino beam before any oscillations occur at the NuMI peak neutrino energy, whilst the Far Detector allows a measurement after oscillations have taken place. Using the Near Detector measurement to construct a prediction of an unoscillated Far Detector spectrum allows for the measurement of the energy dependant deficit of $\nu_{\mu}$, the depth and position of which are dependant on the atmospheric mixing parameters. Crucialy the two detectors are designed to be as similar as possible, such that many shared systematic errors are eliminated when the ratio of the measurements are made.

However, a number of other studies are possible, for example, the NuMI beam can also run in $\bar{\nu}_{\mu}$ mode allowing the same measurements to made with antineutrinos. This enables us to place strong limits on direct CPT violation in the lepton sector outside of $\delta_{c p}$.

The MINOS Far Detector is also $705 \mathrm{~m}$ underground, this shielding from cosmic muon backgrounds allows for the measurement of atmospheric oscillation parameters using neutrinos from cosmic rays in a less effective method analogous 
to that of Super Kamiokande[40], where our magnetic field (discussed in section 3.8.2) gives us an energy resolution for exiting muons which allows us to stay competative with Super Kamiokande despite our far smaller fiducial volume. This complementary measurement of the atmospheric mixing parameters can then be combined with the NuMI $\nu_{\mu}$ disappearance measurement to create tighter still constraints on the atmospheric mixing parameters.

The low energy reconstruction in the MINOS detectors is such that electron tracks and NC showers (discussed in section 3.8.1) are very hard to distinguish. However, whilst not optimised for the identification of $\nu_{e}$ events MINOS can also measure $\nu_{e}$ appearance in the NuMI beam [57]. The MINOS appearance group have produced a number of constraints on $\nu_{e}$ appearance which were highly competitive for their time by using sophisticated electron track identification neural networks.

The MINOS experiment has produced a number of other competitive studies, including searches for sterile neutrinos [58] by looking for deficits in our NC event rate and cross section studies [59] at the Near Detector.

\subsection{The NuMI Neutrino Beam}

\subsubsection{Neutrino and Antineutrino Production}

The core principle of the Neutrinos at the Main Injector (NuMI) [60] beam is the same as a standard neutrino beam [61, the collision of primary protons onto a fixed target to produce secondary mesons which will, in turn, decay to tertiary neutrinos. Figure 3.2 shows an outline of neutrino production in the NuMI target hall. The primary protons are extracted at $120 \mathrm{GeV}$ from the FNAL 
Main Injector (MI) by three horizontal injectors and three Lambertson magnets [62]. The MI uses multi-batch slip stacking [63] to load first 5 batches from the Booster and then 6 more with slightly different profiles. Historically two of these batches would be dedicated to the Tevatron and the remaining nine extracted for NuMI64]. A pulse is produced every 2.2 seconds and every set of batches had between $3.6 \times 10^{12}$ or $40 \times 10^{12}$ protons depending on whether the tevatron was running.

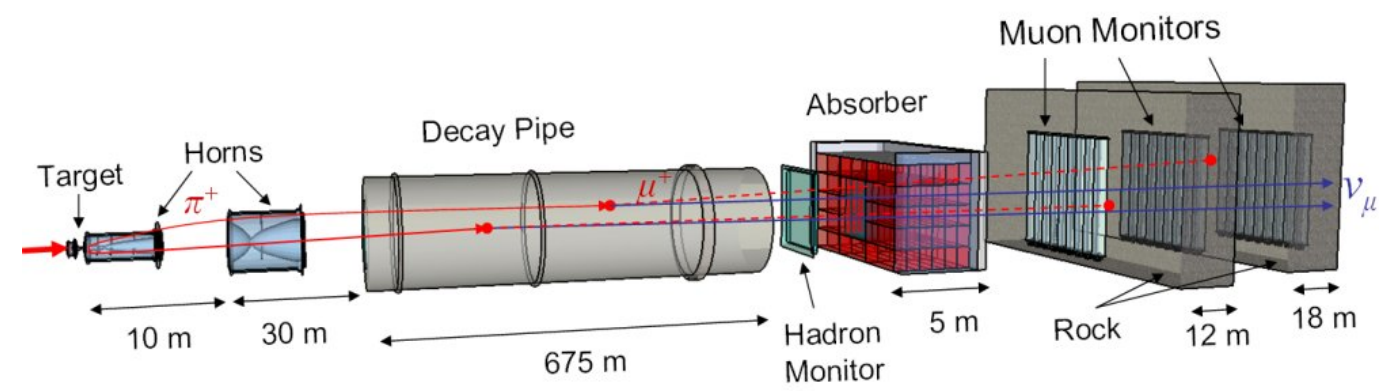

Figure 3.2: Schematic of the NuMI Target hall showing an example muon neutrino production via the decay of a focused $\pi^{+}$.

Those extracted protons are then focused by a series of quadrupole magnets and bent downwards by dipole magnets towards the NuMI target at $58 \mathrm{mrad}$ such that it points directly towards the Soudan Mine. This proton beam travels through position monitors, loss monitors, and proton counters to reach a graphite collimating baffle just before the target which protects the downstream beam components from beam halo damage. Upon reaching the NuMI target itself the proton beam has an RMS of $1.1 \mathrm{~mm}$.

The NuMI target is composed of 47 vertical graphite fins. Each fin is $6.4 \mathrm{~mm}$ wide, $15 \mathrm{~mm}$ tall and $20 \mathrm{~mm}$ long with $0.3 \mathrm{~mm}$ spacing. Arranged longitudinally 
to form a total target length of $954 \mathrm{~mm}$ or approximately 1.9 hadronic interaction lengths. During target scans a 48th fin is mounted horizontally upstream of the main target to help align the beam. Figure 3.3 shows a technical drawing of a NuMI target.

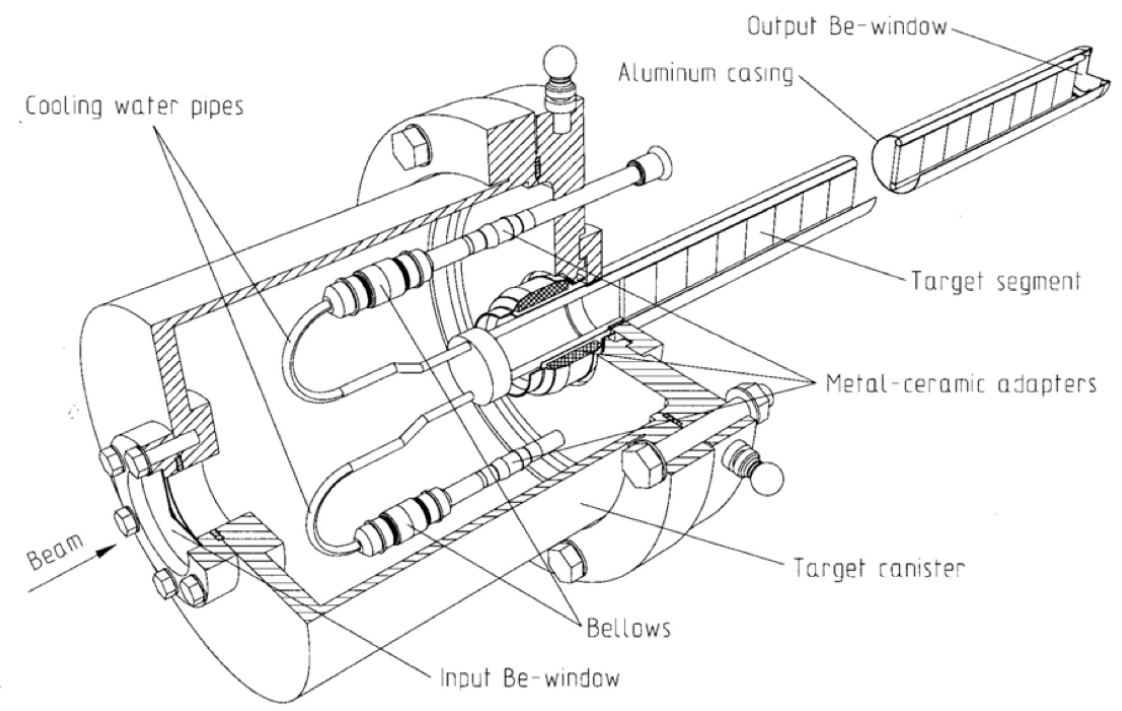

Figure 3.3: Technical drawing of NuMI target and target housing.

A given NuMI target has a limited lifetime. Assuming the target does not develop more serious problems, such as a water leak, the graphite will delaminate over time and lose density in the region of the shower maximum where most of the primary beam energy is absorbed. This ablation of the target will in turn lead to a several percent drop in the final neutrino flux. The NT-02 target installed between Run I and II showed signs of ablation from halfway through Run II (the different run periods considered in this thesis are described in section 5.2).

The primary proton's collision with the target produces a secondary beam of 
unfocused pions and kaons:

$$
p+C \rightarrow h^{ \pm}+X
$$

where $h=(\pi, K)$

This secondary beam is then focused by a pair of magnetic focusing horns [65] so as to enhance the flux of neutrinos seen at the MINOS Near and Far Detectors. In a typical run $185 \mathrm{kA}$ passes along the outer and inner surfaces, or conductors, of the horns in opposite directions to produce a toroidal magnetic field between the horn surfaces with a peak intensity of $2.8 \mathrm{~T}$. This magnetic field selects the mesons produced in the target by both sign and momentum, bending mesons toward or away from the beam axis. In essence, the horns act like point-to-parallel focusing lenses with a focal length proportional to particle momentum because of the parabolic shape of the inner conductors. Any mesons bent towards the axis will have a better likelihood of producing a neutrino which reaches a MINOS detector as the decay neutrino will have a similar forward boost after the decay.

A particular region of tranverse $\left(\mathrm{p}_{\mathrm{T}}\right)$ momentum space is selected by the focusing horns such that the focusing peak is at a particular energy. This peak energy can be changed by altering the relative distance between the two horns, the current flowing through the horns or the relative distance between the first horn and the target. Only the latter technique has been used as the target was designed to be moved without opening the shielding around the target hall. The horns can also be moved, however they are left in the lowest energy configuration where we have sensitvity to neutrino oscillations. The positions relative of the target relative to the first horn breaks down into three main modes, low energy with the target in position at just $0.1 \mathrm{~m}$ upstream, pseudo-medium energy with the target $1 \mathrm{~m}$ upstream, and pseudo-high energy with the target $2.5 \mathrm{~m}$ upstream. 

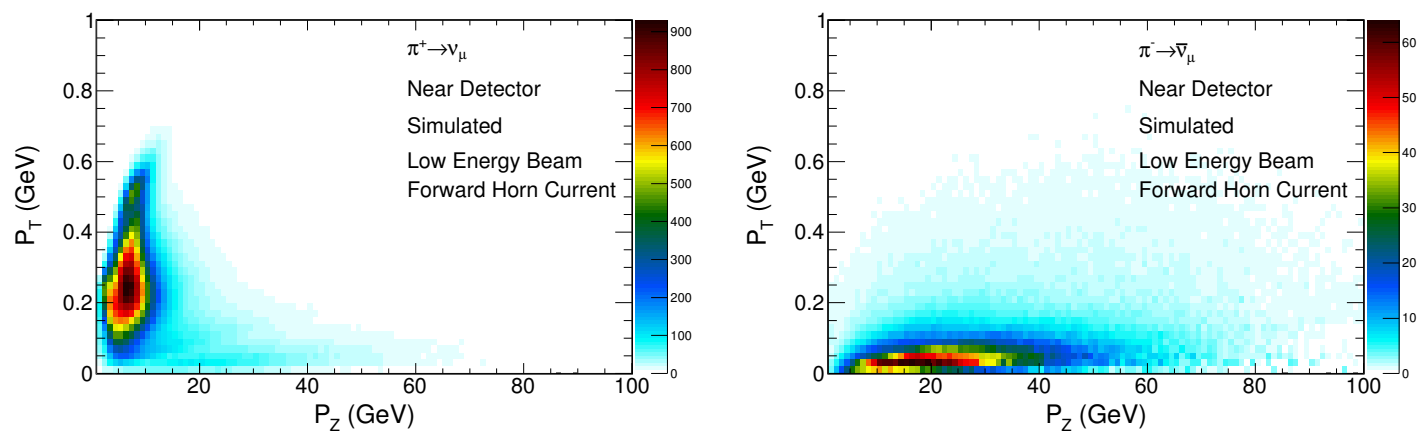

Figure 3.4: The transverse $\left(\mathrm{p}_{\mathrm{T}}\right)$ and longitudinal $\left(\mathrm{p}_{\mathrm{Z}}\right)$ momentum distributions of $\pi^{+}$parents of neutrinos (left) and $\pi^{-}$parents of antineutrinos (right) seen at the Near Detector during low energy forward horn current running. The focused component has a range of transverse momentum and longitudinal momentum is peaked at narrow range of energies, the unfocused component has a broad range of $p_{Z}$ and hence total momentum.

The secondary beam also includes unfocused mesons, with the largest contribution coming from mesons with low transverse momentum which pass through the necks of both horns without being focused or defocused. With a wide range of longitudinal and therefore total momenta these mesons produce a diffuse high energy tail in the final neutrino spectrum. These low transverse momentum mesons are also the primary source of anti-neutrinos in the neutrino beam and neutrinos in an anti-neutrino beam. The field will always focus one charge and anti focus the other. Figure 3.4 shows the $\pi^{ \pm}$parent momentum of neutrinos, seen at the Near Detector, during low energy forward horn current running, and Table 3.1 shows the decays contributing to the neutrino event rate at the MINOS Near Detector.

Once the mesons have been focused they enter a decay volume composed of a $675 \mathrm{~m}$ long $2 \mathrm{~m}$ diameter steel pipe embedded in concrete shielding. The length is chosen because it is approximately the decay length of a $10 \mathrm{GeV}$ pion, and the 
two body decay of the neutrino producing pion decay contributes over $78 \%$ of the neutrino beam composition.

Originally the pipe was also evacuated to approximately 1 Torr so mesons could decay without interaction in the decay pipe. However the aluminium pipe at the upstream end of the decay pipe degraded over time to the point that an implosion was a serious risk and steps were taken to increase the pressure and hence decrease the risk of damage by filling the decay pipe with $0.9 \mathrm{~atm}$ of helium. There are two key changes to the flux, the first being some of the mesons which would have decayed to neutrinos instead interact with the helium so the flux in the focusing peak decreases by a few percent [66]. Secondly, it acts as a second target for meson and hence neutrino production which is completely unfocused, causing a small increase in flux above the focusing peak.

\begin{tabular}{|ll|c|}
\hline & Decay Process & Percentage Contribution \\
\hline$\pi^{+}$ & $\rightarrow \nu_{\mu}+\mu^{+}$ & $78.7 \%$ \\
$\pi^{-}$ & $\rightarrow \bar{\nu}_{\mu}+\mu^{-}$ & $7.2 \%$ \\
\hline$\mu^{+}$ & $\rightarrow \bar{\nu}_{\mu}+\nu_{e}+\mathrm{e}^{+}$ & $1.2 \%$ \\
$\mu^{-}$ & $\rightarrow \nu_{\mu}+\bar{\nu}_{e}+\mathrm{e}^{-}$ & $0.1 \%$ \\
\hline & $\rightarrow \bar{\nu}_{\mu}+\mu^{-}$ & $0.7 \%$ \\
$K^{-}$ & $\rightarrow \bar{\nu}_{e}+\pi^{0}+\mathrm{e}^{-}$ & $0.0 \%$ \\
& $\rightarrow \bar{\nu}_{\mu}+\pi^{0}+\mu^{-}$ & $0.0 \%$ \\
\hline & $\rightarrow \nu_{\mu}+\mu^{+}$ & $10.6 \%$ \\
$K^{+}$ & $\rightarrow \nu_{e}+\pi^{0}+\mathrm{e}^{+}$ & $0.5 \%$ \\
& $\rightarrow \nu_{\mu}+\pi^{0}+\mu^{+}$ & $0.3 \%$ \\
\hline & $\rightarrow \nu_{\mu}+\pi^{-}+\mu^{+}$ & $0.2 \%$ \\
$K_{L}^{0}$ & $\rightarrow \bar{\nu}_{\mu}+\pi^{+}+\mu^{-}$ & $0.1 \%$ \\
& $\rightarrow \nu_{e}+\pi^{-}+e^{+}$ & $0.3 \%$ \\
& $\rightarrow \bar{\nu}_{e}+\pi^{+}+e^{-}$ & $0.1 \%$ \\
\hline
\end{tabular}

Table 3.1: Perecentage contribution of different decays in the target hall to the neutrino event rate in the MINOS Near Detector during low energy forward horn current running after the addition of helium to the pipe. Numbers from truth information in Monte Carlo (MC). 

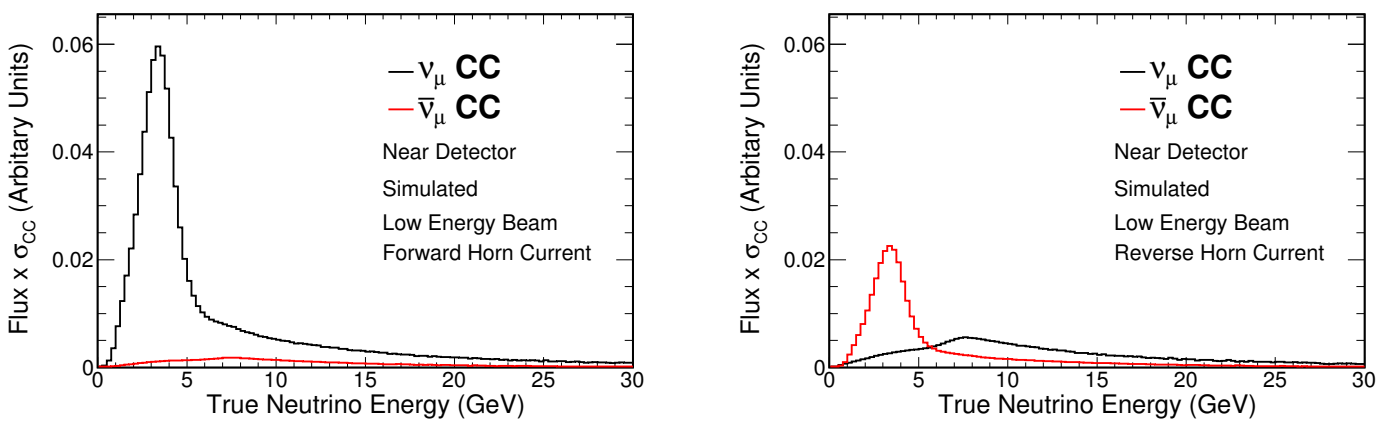

Figure 3.5: The CC $\nu_{\mu}$ (black) and $\bar{\nu}_{\mu}$ (red) event rates at the Near Detector for FHC (left) and RHC (right).

The decay pipe is followed by a beam dump and $240 \mathrm{~m}$ of rock to stop any remaining primary and secondary beam or charged decay products so that only neutrinos remain. Several ionization chambers in this region monitor the remaining hadrons and tertiary muons produced with neutrinos in meson decays.

The final beam is either composed primarily of muon neutrinos or antineutrinos depending on the direction of the current flowing throughout the focusing horns and hence the charge of the focused mesons. In Forward Horn Current (FHC), or neutrino focusing mode, the NuMI beam is $91.7 \% \nu_{\mu}, 7 \% \bar{\nu}_{\mu}$, and $1.3 \% \nu_{e} / \overline{\nu_{e}}$ 's. In Reverse Horn Current $(\mathrm{RHC})$, or anineutrino focusing mode, the NuMI beam is $40 \% \bar{\nu}_{\mu}, 58 \% \nu_{\mu}$, and $\% 2 \nu_{e} / \bar{\nu}_{e}$ 's. The $\nu$ CC event spectra in the Near Detector in FHC and RHC running are shown in Figure 3.5

In both cases the wrong sign neutrino background comes from low transverse momentum mesons which are largely unaffected by the focusing horns. The reason why the overall make up of the beam is still predominantly $\nu_{\mu}$ in RHC mode is that $\nu_{\mu}$ have higher interaction and production cross sections than $\bar{\nu}_{\mu}$. At a momentum of 4-10 GeV (and hence a neutrino momentum of roughly 2-5 
$\mathrm{GeV}), \pi^{+} \mathrm{s}$, which are the primary $\nu_{\mu}$ parents, are produced about $30 \%$ more often than $\pi^{-} \mathrm{s}$, which are the primary $\bar{\nu}_{\mu}$ parents. This effect was measured at the CERN experiment NA49 [67]. Similiary, at CC $\nu_{\mu}$ cross-sections are a factor of two larger than $\bar{\nu}_{\mu}$ with a particularly large difference at smaller energies.

\subsection{MINOS Detectors}

\subsubsection{Overview}

As already discussed, the design of the two MINOS detectors 68 was strongly motivated by a desire to be as similar as possible so that many systematics would cancel out in Far to Near ratios important to many of our analyses. Both detectors are tracking, sampling calorimeters made of alternating planes of $1 \mathrm{~cm}$ thick plastic scintillator, segmented in $4 \mathrm{~cm}$ wide strips and $2.56 \mathrm{~cm}$ thick steel passive absorbers. Figure 3.6 shows how the steel and scintillator form a MINOS plane.

There are, however, some discrepancies in their design motivated by differences in the neutrino flux at the Near Detector $1 \mathrm{~km}$ from the target and the far lower flux at the Far Detector $735 \mathrm{~km}$ from beam start.

\section{Far Detector}

The Far Detector is located $735 \mathrm{~km}$ away from the NuMI beam start and sees about three neutrino interactions per day in a total fiducial mass of 5,400 tonnes. A large fiducial volume is necessary to achieve even that low event rate, as the flux at the Far Detector is exceedingly low.

The detector is an octagonal prism of $8 \mathrm{~m}$ width and consists of 484 octagonal steel planes alternated with scintillator strips. It is split into two larger modules 


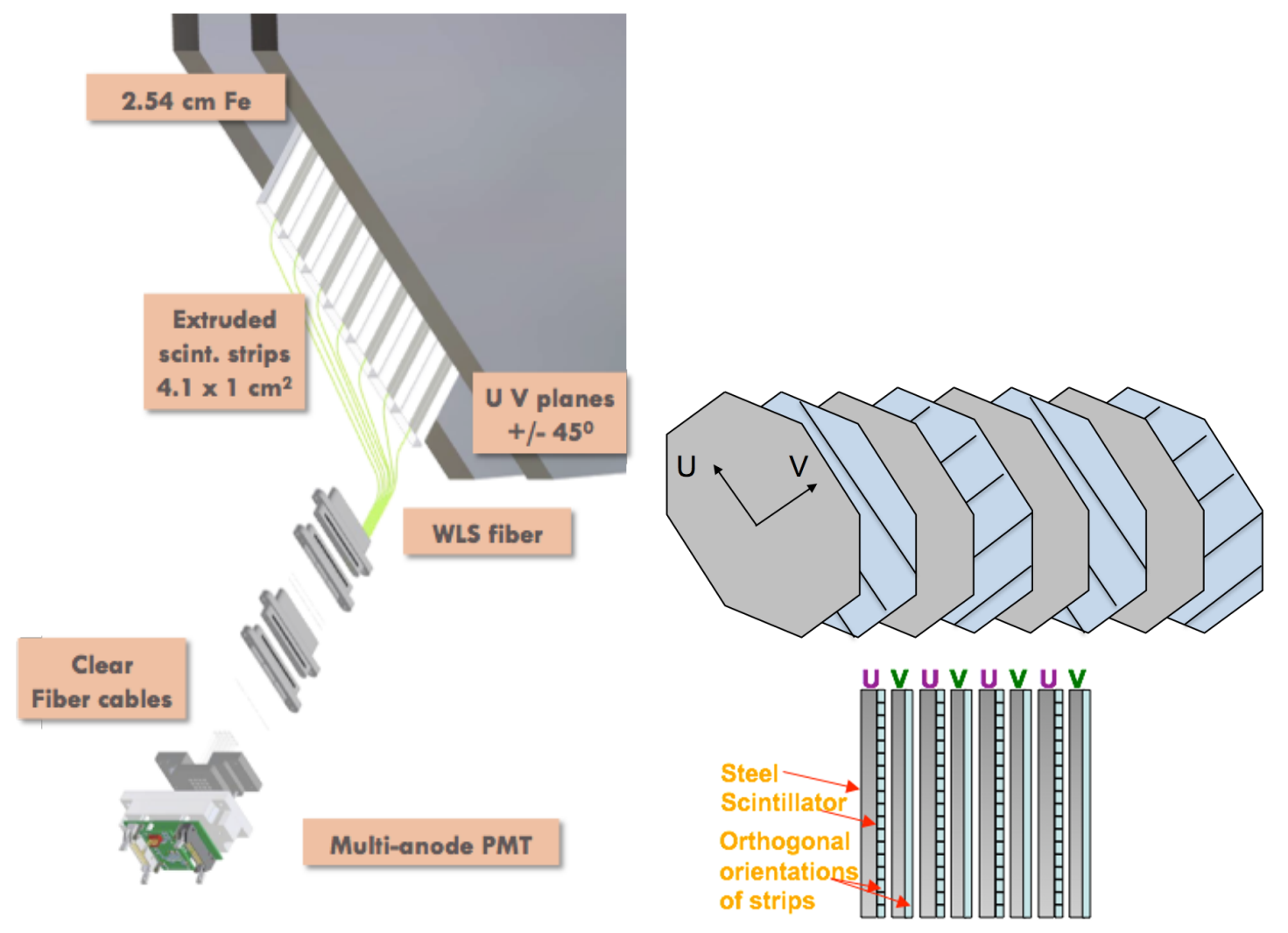

Figure 3.6: Scintillator strips attached to steel planes to make a single MINOS plane (left) and with the strips in an alternating $\pm 45^{\circ}$ angle labelled $\mathrm{U}$ and $\mathrm{V}$ (right) so as to allow full 3D reconstruction. Figures taken from [69]. 
(super modules) of 249 and 237 planes each, with an air gap of $\approx 1.2 \mathrm{~m}$ separating them. The scintillator strips are mounted on the upstream face of each steel strip with the exception of the front plane of each super module. A hole holding current-carrying cable runs through the centre of the detector so as to create a cylindrical magnetic field of $\approx 1.3 \mathrm{~T}$.

The scintillator planes are made of $4 \mathrm{~cm}$ wide strips. Strips on adjacent planes are orientated perpendicular to one another so as to allow three dimensional reconstruction of events. Additionally they are orientated at $45^{\circ}$ des to the vertical defining a co-ordinate system of $\mathrm{U}$ and $\mathrm{V}$ whilst this thesis will use the coordinate system of $\mathrm{X}$ and $\mathrm{Y}$ as defined as horizontal and vertical relative to the beam. In both cases the beam direction is referred to as $\mathrm{Z}$.

The steel thickness means that the stopping distance of a typical $2 \mathrm{GeV}$ muon from a CC $\nu_{\mu}$ interaction is $3 \mathrm{~m}$ or 50 detector planes. The octagonal shape resembles a cylinder whilst making each steel sheet easy to manufacture.

\section{Near Detector}

The Near Detector is located $110 \mathrm{~m}$ underground and sees about ten neutrino interactions per proton spill in a total fiducial mass of 980 tonnes. Even with a much lower fiducial mass the Near Detector sees orders of magnitude more events than the Far Detector in a day, this leads to a number of differences in its design starting with a much lower mass. The shape is a vertically compacted, 4.8 $\mathrm{m}$ by $3.8 \mathrm{~m}$, octagon with the coil hole off centre. The coil hole is deliberately off centre, $1.48 \mathrm{~m}$ horizontally displaced from the beam axis, so as to create a region where the magnetic field is exactly the same as that of the Far Detector which is centred on the beam axis. This oversaturation of the beam side of the 
detector limits the occupancy of the strips on the other, less interesting, side of the detector.

The detector is constructed out of 282 steel planes. All of the first 120, except the very first, have scintillator mounted on them and, of those, every fifth plane is completely covered with scintillator the rest have only a smaller region around the beam axis covered. Out of the remaining planes every fifth plane is fully covered with scintillator, the rest have no scintillator at all. This design is such that the highly instrumented front part can be used for hadronic shower energy measurements whilst the partially instrumented back is only used for muon tracking.

\subsubsection{Steel}

The average steel density is $7.85 \pm 0.03 \mathrm{~g} \mathrm{~cm}^{-3}$ [69]. The RMS deviation of the plane masses is $0.35 \%$ such that the plane thickness varies by $0.35 \%$.

\subsubsection{Scintillating Strips}

The most basic component of the MINOS scintillator system is a strip. Each scintillator strip is made from extruded Dow STYRON polystyrene which has been doped with organic scintillator PPO fluor (1\% by weight), the scintillator POPOP ( $0.03 \%$ by weight), and coated with a $2 \mathrm{~mm}$ reflective layer of $\mathrm{TiO}_{2}$ (15\% by weight)[70]. The fluor doped polystyrene produces scintillation photons, scintillator light, when excited by ionising radiation. The reflective layer traps and reflects the scintillator light so that each strip is optically isolated from its neighbour.

Charged particles traversing the detector deposit energy in both the steel planes 
and the scintillator strips. Scintillator light is internally reflected within the strip until it is absorbed by Y-11 fluor in the $1.20_{-0.01}^{+0.02} \mathrm{~mm}$ wide wavelength shifting fibre (WLS) that runs down a groove on one side of each strip. This fibre shifts the scintillator light from blue $(\lambda \approx 420 \mathrm{~nm})$ to green $(\lambda \approx 520 \mathrm{~nm})$ and transports it to the end of the strips. By shifting the scintillator light the WLS minimises self absorption as the absorption spectrum of the Y-11 fluor barely overlaps with its emission spectrum.

The scintillator strips are grouped into modules of 16 and 24, and each module is wrapped in aluminium to make it light tight and provide protection during installation. These modules are then attached to the steel plates to build up each steel-scintillator plane. Figure 3.7 shows an illustration of a single scintillator strip, Figures 3.8 and 3.9 show how those strips are arranged in modules at the Near and Far Detector. 


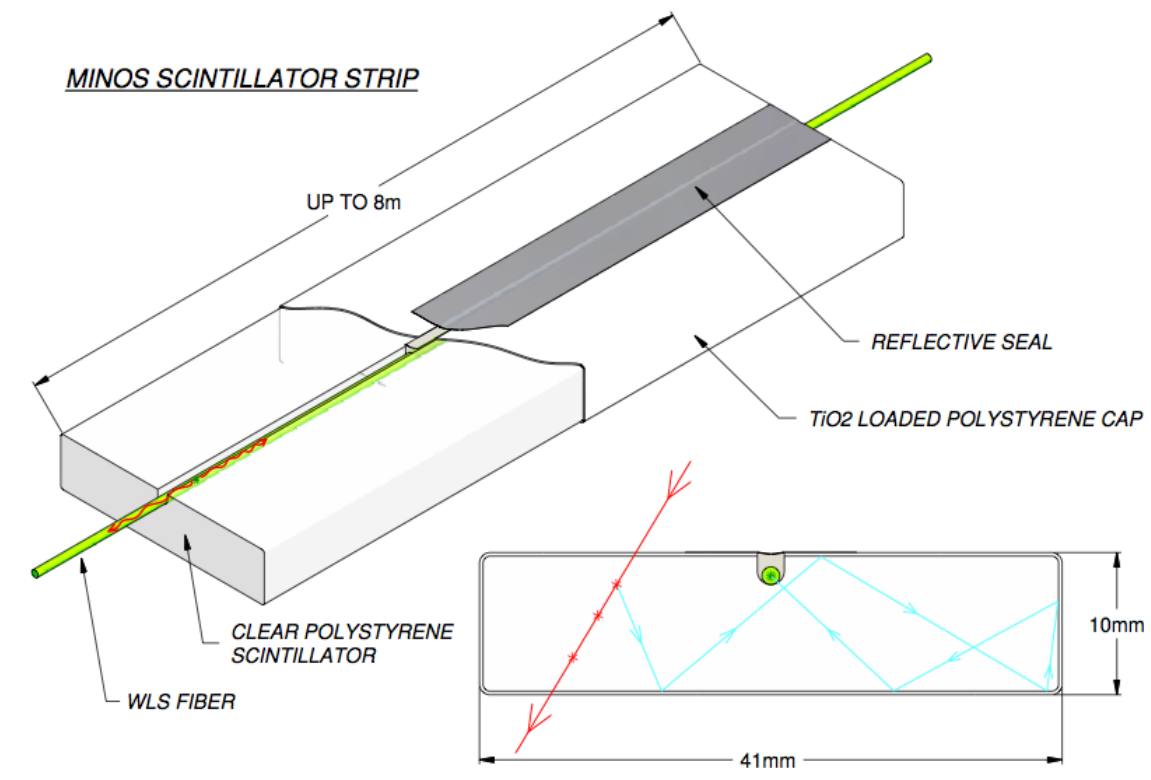

Figure 3.7: Technical drawing of an example scintillator strip. Figure taken from [69]. 

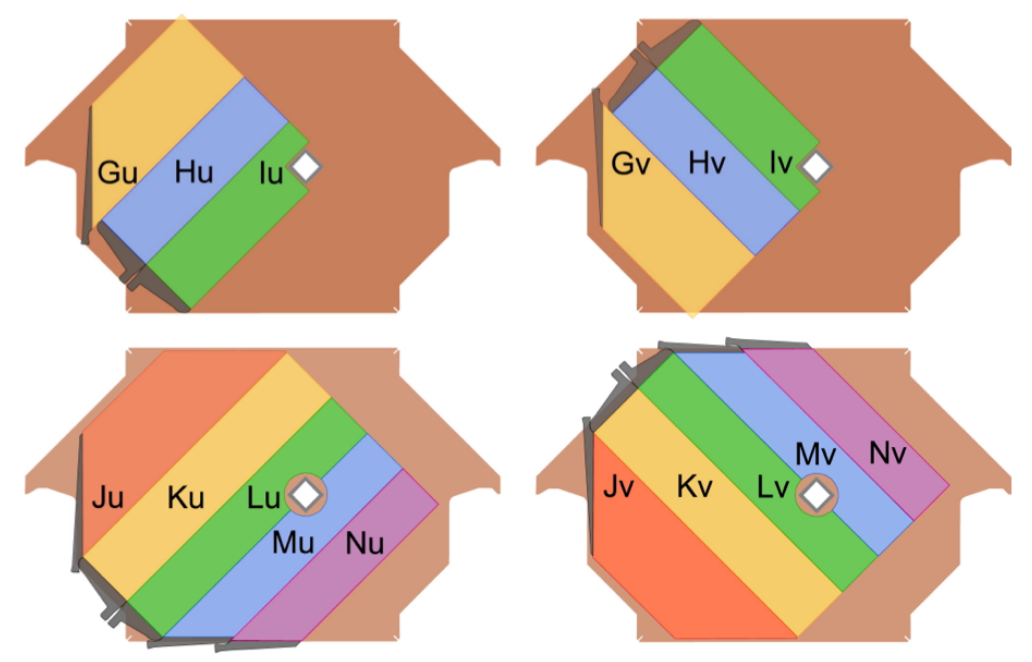

Figure 3.8: Scintillator module arrangement in the Near Detector planes. The four configuration are partial $\mathrm{U}$ (top left), partial V (top right), full $\mathrm{U}$ (bottom left) and full V (bottom right). Every fifth plane is alternately a full $\mathrm{U}$ or V plane. In the upstream calorimeter intervening planes are alternating partial $\mathrm{U}$ and $\mathrm{V}$ planes with the area near the beam centre, left of the coil hole, instrumented. In the downstream calorimeter the intervening planes are left unistrumented. Figures taken from [69].

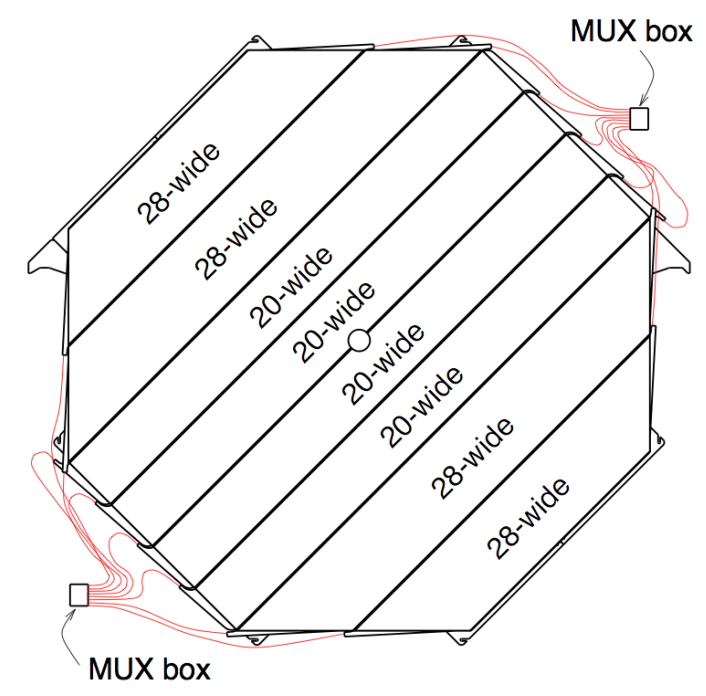

Figure 3.9: Scintillator arrangement in a Far Detector plane. Every plane in the Far Detector is fully instrumented. Figure taken from [69]. 


\subsubsection{Photomultiplier Tubes}

The scintillator light from the WLS is transported by clear optical fibres to Hammamatsu multi-anode photomultiplier tubes (PMTs). 16-anode PMTs with gains of $\approx 1 \times 10^{6}$ are used at the Far Detector, and 64-anode PMTs with gains of $\approx 0.8 \times 10^{6}$ are used at the Near Detector. A voltage of $\approx 800 \mathrm{~V}$ is required to achieve those gains.

The voltage and gains listed here are approximate as the real gain varies between PMT tubes, with the voltage set such that the highest gain pixel in each pixel tube is at $\approx 1 \times 10^{6}$ at the $\mathrm{FD}$, and such that the average pixel gain within each tube is $\approx 0.8 \times 10^{6}$ at the ND. At the Far Detector this results in a mean voltage of $763.9 \mathrm{~V}$ with an RMS of $41.81 \mathrm{~V}$ and a mean gain of $0.79 \times 10^{6}$ with an RMS of $0.11 \times 10^{6}[71$. At the Near Detector this results in a mean voltage of $812.7 \mathrm{~V}$ with an RMS of $23.84 \mathrm{~V}$ and a mean gain of $0.82 \times 10^{6}$ with an RMS of $0.17 \times 10^{6}[72]$. Additionaly the gains vary with time and temperature, and the voltages are continually adjusted to acheive close to nominal gains with the remaining differences accounted for during calibration (see Section 3.6).

The PMTs are stored in light tight steel crates which also provide shielding from the detector's magnetic field. Figure 3.10 shows how the WLS fibres readout to a PMT. 

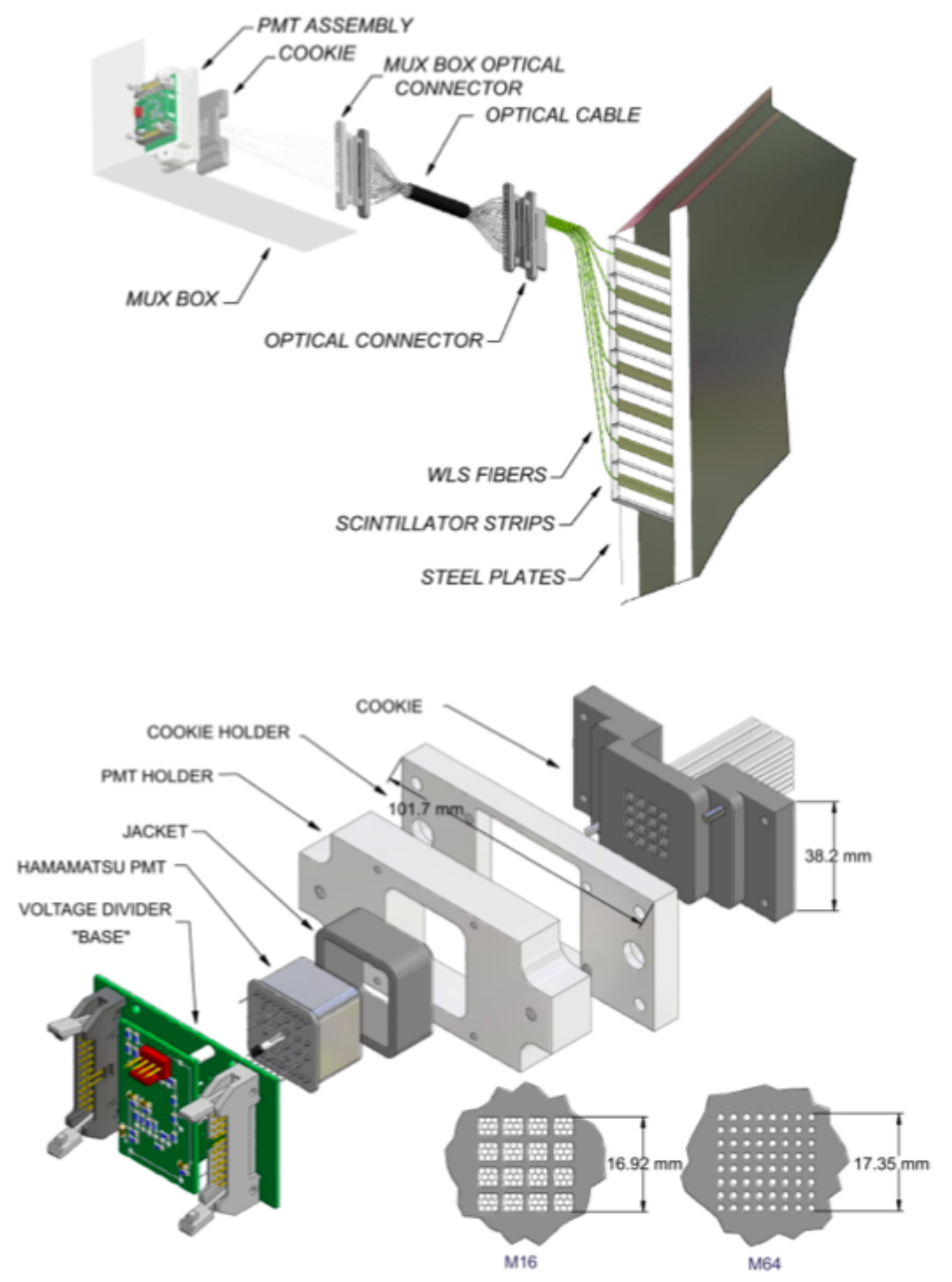

Figure 3.10: Technical drawing showing how WLS fibres from multiple strips are grouped and sent to a multi-pixel photo multiplier tube. In the Far Detector eight strips go to each of 16 PMT pixels, in the Near Detector a single strip goes to each of 64 PMT pixels. Figure taken from [69]. 
At the Far Detector 8 scintillator strips, from a single detector plane, are read out by a PMT anode. The strips are separated by about $1 \mathrm{~m}$, so as to aid in resolving any ambiguities. Information from adjacent planes is used during reconstruction to identify the actual strip hit. Additionally the pattern the strips are connected in changes between successive planes to further aid in this disambiguation.

In the first 120 planes of the Near Detector each scintillator strip reads out to a single unique anode. In the remaining planes four strips separated by about $1 \mathrm{~m}$ on a single plane read out to a single anode.

Because the PMTs have a multi-anode design electric charges can drift within the PMT, registering as a signal on the incorrect, nearest-neighbour, anode. This shifted signal is referred to as "cross talk" and can cause a low pulse hit (with a signal size below the mean amplitude corresponding to one photoelectron) to be associated with a scintillator trip where there is no true activity.

\subsubsection{Magnetic Field}

In addition to being a low cost source of fiducial mass the steel component of the MINOS planes allows us to magnetize the detectors. Each of the two detectors has a coil which passes through a hole through the full length of each detector. Each Far Detector super module is magnetized separately using a water cooled 190-turn coil made from Teflon insulated stranded copper wire which carries 15.2 $\mathrm{kA}$ per turn to produce an average magnetic field strength of $1.42 \mathrm{~T}$ in the fiducial volume. The Near Detector coil instead has 8 turns, each made up of six cold-conformed aluminum conductors carrying current in parallel. Due to the high currents involved, there are no PMTs on the coil return side of the Near 

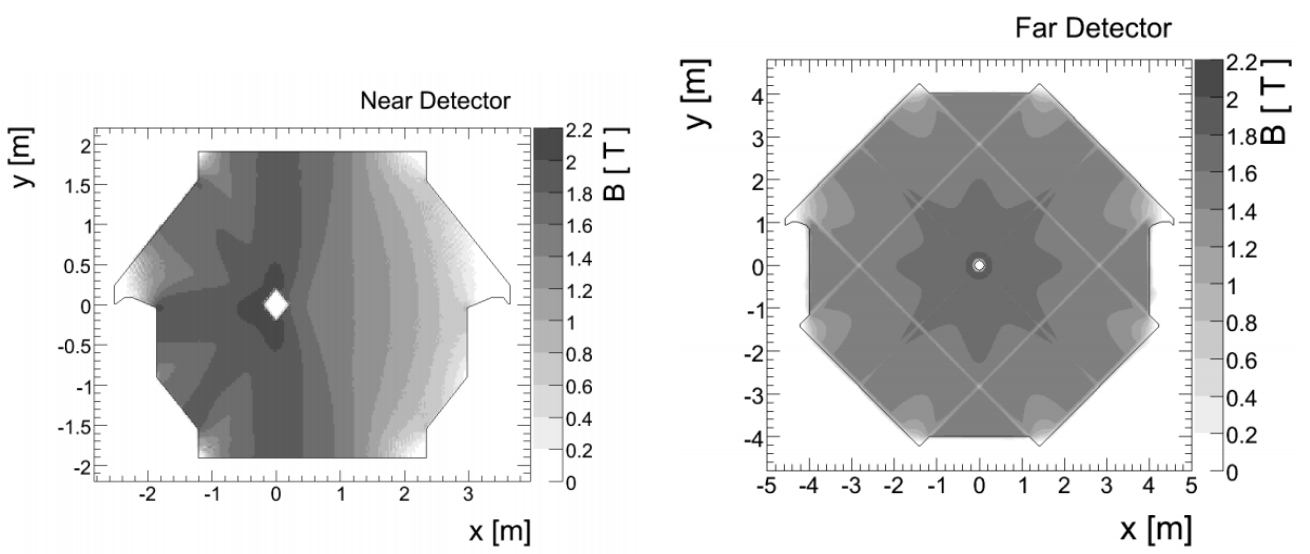

Figure 3.11: Maps of the magnetic field strength in the MINOS Near (left) and Far (right) detectors. Over the fiducial volume the average field strength is 1.42 $\mathrm{T}$ in the Near and 1.28 $\mathrm{T}$ in the Far Detector. Figures taken from [69].

\section{Detector.}

The magnetic fields in the detectors allow us to measure the momentum of muons in the detector even if they leave the detector before ranging out (discussed in section 3.8.2) as the curvature of the track's of any muons in the toroidally magnetised detector is proportional to the ratio of the tracks electric charge to its momentum, $q / p$. It also makes possible the measurement of the electric charge of individual muons, and as neutrinos and anti-neutrinos produce muons of opposite electric charge in $\mathrm{CC}$ interactions this means the MINOS detectors can distinguish between neutrinos and antineutrins on an event-by-event basis. The polarity of the magnets can also be switched in RHC mode so that positive muons from $\bar{\nu}_{\mu}$ are focused into rather than out of the detector. This electric charge sign identification is the most unique aspect of the MINOS experiment and has made many novel analyses possible, including our anti-neutrino only atmospheric mixing angle measurement [51] and our $\nu_{\mu} \rightarrow \bar{\nu}_{\mu}$ transitions analysis [3]. Figure 3.11 shows the magnetic field map for the Near and Far MINOS Detectors. 


\subsubsection{Electronics and Readout}

Near and Far Detector electronics differ as the low event rate at the Far Detector means very rarely will two neutrino events occur at far detector within a narrow spread of time. Therefore at the far detector a greater amount of time where the detector is dead to a new event whilst reading out the last event can be tolerated (deadtime).

\section{Far Detector}

At the Far Detector[74] each PMT is read out by a single Viking (VA) chip. 22 Channels on each chip are used, 16 for reading-out of the 16 anodes of the PMT, one is used for reading out the PIN diode monitoring the light injection system, and five for performing noise subtraction.

Additionaly, a signal from each PMT is sent to a chip called an ASDLite which will produce a signal if the PMT registers more than $\sim \frac{1}{3}$ the mean amplitude corresponding to one photoelectron. This signal is then sent to a VARC (VA Readout Controller) which controls 36 VA chips and provides the lowest level trigger for readout of a VA chip and for an ETC (Event Timestamp Controller) to timestamp the readout signal.

The VA readout signal is digitised by an ADC which is part of a VMM (VARC Mezzanine Module) which is a module intermediate to the VARC and VA chip. Each VMM, and therefore ADC, is responsible for six VA chips and digitises each in sequence. Digitisation of a VA chips takes $\approx 5 \mu$ s so if all six need to be read out it will take $\approx 30 \mu \mathrm{s}$, during which all six chips will be dead to new signals.

These VARCs are read out by a VME computer with two buffers such that one 
buffer can be written to whilst the other is read. The VME computers then send data to the Data Acquisition (DAQ) system.

\section{Near Detector}

Every beam spill, lasting up to $10 \mu$ s can produce of order 10 neutrino events in the Near Detector[75]. In order to facilitate reconstruction of all of those events the Near Detector electronics are designed to be fast and to have no deadtime. The shortest time that needs to be resolved is that between the buckets of protons which make up one proton spill. The size of these buckets is determined by the frequency of the accelerator's RF cavities which is $53 \mathrm{MHz}$, accordingly the Near Detector electronics are designed to record signals with a deadtimeless granularity of $18.8 \mathrm{~ns}$.

The signal from each PMT anode feeds into a electric charge to current encoder (QIE). In the QIE the signal first hits a current splitter which divides the current into eight ranges, a set of eight capacitors which are each sensitive to a different range of currents. All capacitors below the one corresponding to the signal are saturated and the current in the first unsaturated range provides a precise measurement of the signal. This variety of capacitors means a better dynamic range for the readout. In a single $53 \mathrm{MHz}$ clock cycle the current splitter integrates all the current, with the first unsaturated range being selected in the next cycle. In the next cycle the analogue signal is converted to an eight bit digital signal by an Analogue to Digital Converter (ADC). A final, fourth, cycle is needed to reset the capacitors in the current splitter. To avoid the readout being dead for the three clock cycles following the integration stage there are four current splitters residing on each QIE, each integrating in sequence for one cycle, all using the same range selector and ADC. 
The 8 bit output from the ADC is extended to 13 bits with the addition of bits which encode the range of the current splitter, as well as which of the four current splitters were used. This string of bits is received by a 1000 string first in first out (FIFO) buffer, which is enough to buffer all strings in a $10 \mu$ s spill.

The QIE is mounted on a board called a MENU (MINOS Electronics for Neutrinos) module with the ADC, FIFO, and a DC current injector which can insert a false signal into the QIE for calibration. Sixteen of these MENU modules are mounted on one MINDER (MINOS Near Detector Electronics Readout) module which both records the time of the signals and controls the modes of the MENUs (initiating the calibration mode, etc.). Each MINDER reads all outputs from the MENU FIFO in sequence and then sends them to a FIFO on a MASTER (MINOS Acquisition, Sparsifier and Timestamper for Event Readout).

Each MASTER module contains eight MINDERs, data from these MINDERs are read into two buffers and then read out by VME computers, as in the Far Detector, with two buffers per MINDER to allow simultaneous reading and writing.

\subsection{Light Injection}

To enable calibration of the PMTs each detector has a light injection system which allows varying pulses of ultra-violet light from LEDs to be shone onto the WLS fibres as they exit the scintillator, simulating light output from the scintillator. The intensity of this light is monitored by PIN diodes that receive light directly from the LEDs and produce a similar signal shape as a PMT and can be read-out using the standard detector electronics. 


\subsection{Triggering}

Detector readout is triggered by each beam spill when a signal or spill trigger is sent to each detector. Readout is triggered for $100 \mu$ s around the spill at both detectors, and at the Near Detector there are no other triggers. At the Far Detector however dark noise in the PMTs and WLS fibres (caused by spontaneous light emission in the fibres[76]) is too high, $\approx 5 \mathrm{kHz}$, and a low level " $2 / 36$ " trigger is applied in any readout period. This trigger is referred to as " $2 / 36$ " as it requires a VARC to receive signals from two PMTs within $\approx 400$ ns of one another and is sufficient to reduce dark noise to a very low level.

Between beam spills cosmic muons can set off an offline trigger based on either a minimum energy deposit in a set of planes or a certain number of planes within one group of planes being hit.

\subsection{Calibration}

Raw detector pulse heights are converted to a fully corrected signal consistent across time, position in the detector, and detector by a multi-step calibration chain 69] [77]. Charge injection and light injections are used to measure the photodetectors and readout systems. While cosmic ray muons are used to measure the scintillator response. Bench top measurement are also used for WLS fibres calibration. A summary of the full chain is:

$$
Q_{\text {corr }}=
$$

$Q_{\text {raw }}(s, x, t, d) \times D_{P M T}(t, d) \times L\left(s, d, Q_{\text {raw }}\right) \times D_{\text {scint }}(t, d) \times S(s, t, d) \times A(s, x, d) \times M(d)$

where $s$ is the strip hit, $x$ is the position in the detector, $t$ is the time of the hit, $d$ is the detector where the hit occurred. $Q_{\text {raw }}$ is the raw pulse height from the strip 
and $Q_{\text {corr }}$ is the fully corrected signal to be passed on to reconstruction where the corrected PMT responses or pulse heights are used to reconstruct physical objects and the pulse heights are interpreted as physical energy deposits. Detailed below are the individual steps in the calibrations process.

\section{$\mathrm{D}_{\mathrm{PMT}}(\mathbf{t}, \mathbf{d})$}

PMT and Electronics drift calibration, where drift is the change in PMT and electronics gain as a function of time. Short term variation is largely a function of temperature and is eliminated with good climate control in the detector buildings. Long term variation comes from both seasonal climate changes and component aging such that there is an $\approx 4 \%$ annual change in the overall detector gain.

This variation in detector gain is calibrated using the LI system. LI data is collated on a monthly basis at each detector to produce an average response per photoelectron per channel. This average response per photoelectron of known energy can then be converted into a gain and used to both calibrate out changes and to tune the voltage across the pmt to stay close to the nominal gain [78].

\section{$\mathbf{L}\left(\mathbf{s}, \mathbf{d}, \mathbf{Q}_{\text {raw }}\right)$}

Linearity, the correction of the output of the electronics such that it is linearly related to the true illumination. At the Near Detector this is acheived by the seperate calibration of the PMTs and the electronics[77]. Linearity of PMT response is calibrated using an extended LI run conducted on a monthly basis where each strip end is pulsed 1000 times at a range of light levels, such that PMT/electronics response as a function of true energy is measured and the gain then linearised offline. Linearity of electronics response is calibrated by injecting 
known electric charge into the each integrator/digitizer and observing the output signal.

The Far Detector electronics and PMTs only experience nonlinearity at higher pulse heights, with $5-10 \%$ non-linearity above $\approx 100$ photoelectrons. Additionaly the Far Detector electronics have a nonlinearity response of a similiar scale to the PMTs such that it is convenient to linearize both with a single correction based on the LI system.

$\mathrm{D}_{\text {scint }}(\mathbf{t}, \mathbf{d})$

Scintillator drift means the scintillator response changes as a function of time and temperature. Nominaly we would expect 2-10 photoelectrons to be produced on each side of a scintillator strip by a minimally ionising particle passing through [69]. $2 \%$ decreases in light levels annually and larger short term variations correlated with temperatures have been observed. Calibration is achieved using a sample of stopping muons at each detector, with the truth amount of energy deposited expected to stay constant over time. The total pulse height per plane deposited by these muons within the middle window of their track is used so as to avoid the non linear spike in $d E / d X$ in the final $10 \%$ of the track. Figure ?? shows the true energy lost per scintillator plane $(\mathrm{MeV})$ for a cosmic muon. 


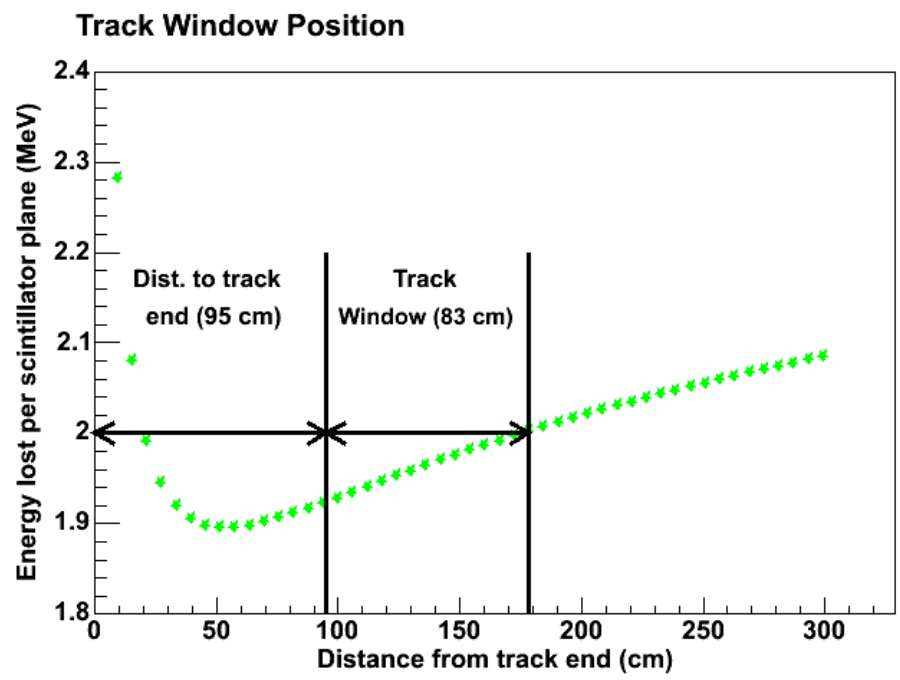

Figure 3.12: Shown is the true energy lost per scintillator plane for a MC cosmic muon and the window in track length used for calibration. Figure taken from [69].

Calibration then comes from comparing the average response over the entire detector at time $t_{0}$ to that recorded on day $t$ :

$$
D_{\text {scint }}(t, d)=\frac{\text { Median response }\left(d, t_{0}\right)}{\text { Median response }(d, t)}
$$

Reasonable statistics, $\mathcal{O} 1000$ stopping cosmic muons per month when averaging over the whole detector, are available at both the ND and FD as the competing effects of detector depth underground and fiducial mass combine to give similiar exposures over time.

$\mathbf{S}(\mathbf{s}, \mathbf{t}, \mathbf{d})$

Detector response also varies on a strip by strip level as a function of time, with some individual strip ends varying from the detector average by as much as $30 \%$. Cosmic ray muon tracks are again used, with the mean light level at each strip end due to cosmic muons being measured after linearisation. Hits are then corrected for known WLS attenuation effects and the path length of the muon such that each 
strip is calibrated for an idealized muon travelling at normal incidence (orthoganl to the $\mathrm{U}$ and $\mathrm{V}$ planes of the dector) through the centre of the strip. The final strip response calibration then is calculated by comparing the strip end response to the detector response:

$$
S(s, t, d)=\frac{\text { Median detector response }(d, t)}{\text { Median strip end response }(s, d, t)}
$$

$\mathbf{A}(\mathbf{s}, \mathbf{x}, \mathbf{d})$

Hits close to the edge of the detector have a higher light level than hits deeper in the detector because of attenuation in the WLS fibre. Accurate maps of attenuation in the fibre were made at the production factory using a known photon source and used to fit a double exponential in each strip:

$$
A(s, x, d)=A_{1}^{d, s} e^{\frac{-x}{L_{1}^{d, s}}}+A_{2}^{d, s} e^{\frac{-x}{L_{2}^{d, s}}}
$$

where $x$ is the length along the strip and $L_{1}^{d, s}$ and $L_{2}^{d, s}$ are the two attenuation lengths. When checked with through-going muons this correction was found to be consistent within $4 \%$

\section{$\mathbf{M}(\mathbf{d})$}

Earlier calibration produces consistent data within each individual detector, in order to make the data in each detector fully equivalent to one another a final step is required. Stopping cosmic ray muons are used to calibrate energy deposition by correcting to their expected deposition. They are chosen as they produce well understood energy depositions, particularly in the $0.5-1.1 \mathrm{GeV}$ range where $\frac{d E}{d x}$ is ₹constant. Additionally, the "track window technique" where only the portion of the muon track is in that energy range reduces a $2 \%$ uncertainty in the track end position to a $0.2 \%$ uncertainty in the energy deposition. This final calibration brings the inter detector consistency to better than $2 \%$. 


\subsubsection{Absolute Track and Shower Energy}

With the hit-to-hit response within the detectors identical, the next step is to evaluate their response to physical events. In order to do this the MINOS calibration detector, CalDet[79], was exposed to a series of well understood test beams at CERN. Pions, kaons, electrons, muons and protons from between 200 $\mathrm{MeV}$ to $10 \mathrm{GeV}$ were tested and compared to GEANT3[80] so as to both benchmark detector response but also quantify the performance of the detector simulation/reconstruction (discussed in section 3.8.2).

\subsection{Simulation}

\subsubsection{Beam Simulation}

MINOS uses the FLUGG 81] 82] MC generator to simulate the NuMI beam so as to incorporate a detailed $G E A N T 4[83$ geometry into a $F L U K A[84$ simulation of the hadronic production, decay, and transport processes. The simulation mainly deals with the secondary meson beams production and its decay to produce neutrinos. Beginning with $120 \mathrm{GeV}$ protons impinging a graphite target and then following any secondary mesons produced as they transport through the focusing horns and into the decay pipe until the moment a particle decays to produce a neutrino or striles the end of the decay pipe. Whenever a particle does decay to produce a neutrino its parents properties are recorded.

We know that the neutrino production is approximately uniform in all directions, hence the position and momentum of the parent is used to draw a solid angle out to our detectors, and the neutrino reweighted and energy adjusted accordingly to give a prediction of our Near and Far Detector flux. This saves vast amounts of processing time by forcing every simulated neutrino to go to the 
detector then reweighing rather than simulating our beam in full until we had statistics comprable to data at our Near and Far Detectors.

We also use a technique called "importance sampling" and "weighting" to reduce the number of low energy particles which need to be simulated. Without any intervention the simulation produces many more low energy mesons than high energy ones, as one might expect. However, this makes it hard to accumulate statistics at higher energies. To compensate two measures are taken, firstly, a $1 \mathrm{GeV}$ tracking threshold is included as any neutrinos produced would be 500 $\mathrm{MeV}$ or lower, which is the lower threshold of the MINOS detector sensitivity. The second is that pions below $30 \mathrm{GeV}$ are importance sampled and weighted using an energy dependant metric such that a fraction of the events are thrown away but the remaining events are given a weight larger than 1 to compensate. The weight is calculated as follows:

$$
W=\frac{30}{\left|p_{\text {total }}\right|} \mathrm{GeV}
$$

$\mathrm{W}$ is the weight and $\left|p_{\text {total }}\right|$ is the total momentum of the particle. Weights are constrained such that they can never go below 1 or above 100 to avoid any one event becoming too important.

Data-to-simulation discrepancies are observed in the Near Detector, however we are able to use a powerful beam reweighting system so that the flux better describes our observation. To do this we reweight the flux as a function of the properties of the neutrino parents as they leave the target. The nature of this reweighting is determined using a simultaneous fit to the many MINOS beam configurations so that the data samples different regions of the $p_{T}-p_{Z}$ space. This ensures that the hadron production reweighting can be better tuned by making use of the full $p_{T}-p_{Z}$ from fixed target experiments [85]. Additionally 
both neutrino and antineutrino samples are used, and we make use of NA49 to constrain the pion electric charge ratio. Ultimately discrepancies which start as large as $30 \%$ in the tail are brought into good agreement. Figure 3.13 shows an example of data/MC agreement at the Near Detector before and after beam reweighting.
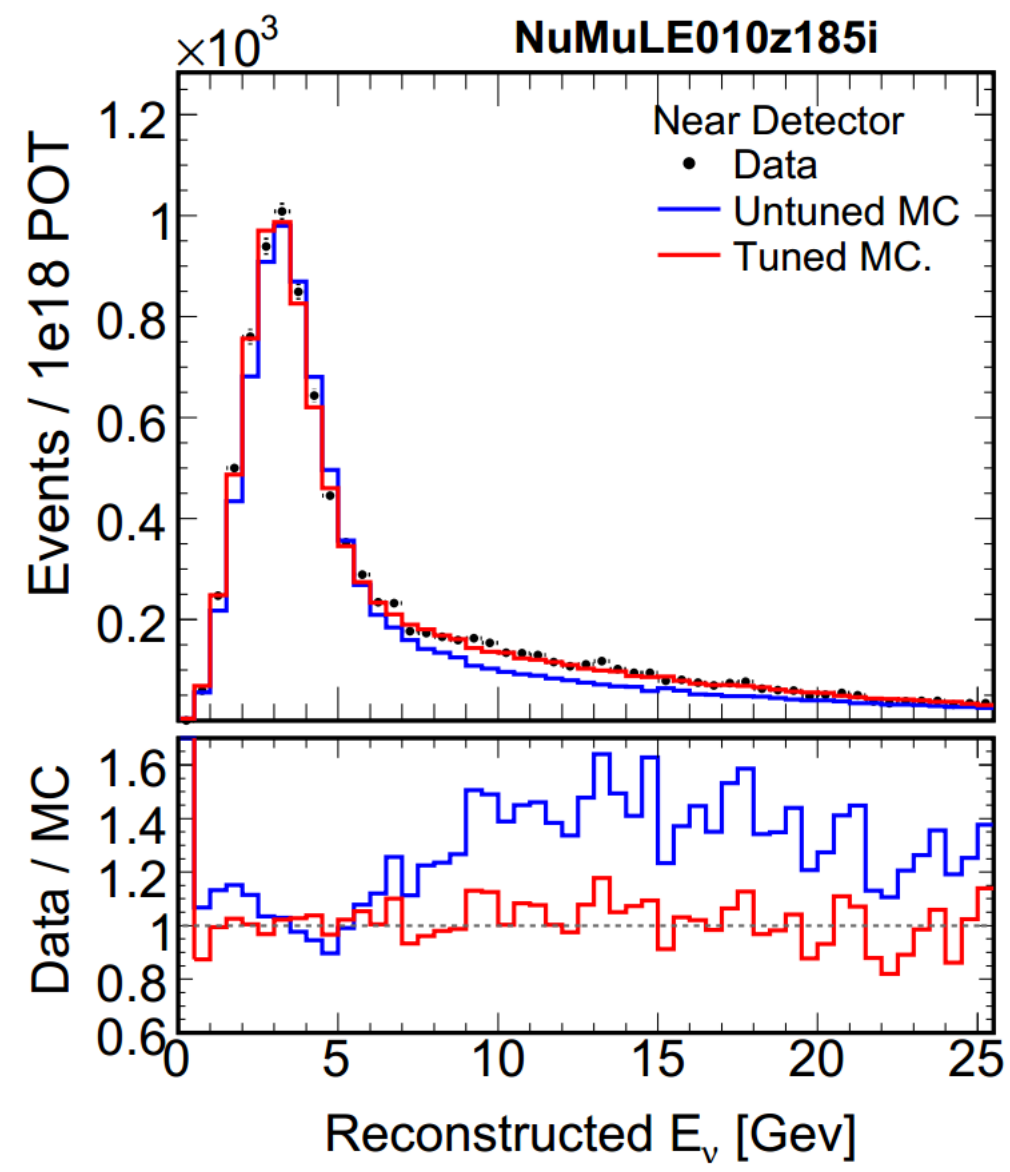

Figure 3.13: Data (black), untuned MC (blue) and tuned MC (red) $\nu_{\mu}$ CC spectra at the MINOS Near Detector during a low energy forward horn current run. Figure taken from [85].

\subsubsection{Detector Simulation}

Simulation of our detectors in MC begins by sampling neutrinos from the above beam simulation using rejection-sampling (pseudo random number sampling) to 
account for the importance weighting. These neutrinos are traced through models of the Near and Far Detector halls so that the neutrinos can interact in both the detector and in the surrounding material.

Neutrino interactions are then generated using the NEUGEN [86] program which simulated both quasi-elastic and inelastic neutrino interactions at a wide range of energies $(100 \mathrm{MeV}$ to $100 \mathrm{GeV})$. NEUGEN simulates hadronization with a combination of PYTHIA/JETSET[87] at high hadronic invariant masses and the $K N O$ [88 phenomenological model at low invariant masses with a smooth transition between the two (referred to as the $A G K Y$ model[89]). NEUGEN also covers the interaction of hadronic particles as they leave the nucleus with the INTRANUKE[90] model of internuclear scattering.

After the products of the interaction have left the nucleus their simulation is taken over by GMINOS. GMINOS includes a detailed geometric model of the detector made in GEANT3 and the magnetic field (built using finite element analysis and measured B-H curves in steel samples). GMINOS transports the particles through the detector geometry and records the strip-by-strip energy depositions as they lose energy into the steel and scintillator. To simulate the high Near Detector event rate multiple interactions in both the detector and the surrounding material are overlaid into single simulated snapshots.

After energy has been deposited in scintillator strips a $\mathrm{C}++$ program called PhotonTransit generates photons in the scintillator based on the GMINOS energy depositions and transports those photons into the WLS fibre and onto the PMT cathode where they are converted into photoelectrons. The program includes the detailed behavior of PMTs and electronics, including non-linearity, noise, cross 
talk, and triggering. Simulation of the real world detector is achieved by applying an inversion of the calibration constants such that it contains the best information about light-levels, attenuation, non-linearity, and gains. Every simulated run is made with a date from a period during actual data taking and calibration constants from that time, and when calibrations are re-applied the Monte Carlo is re-calibrated using the same date as that used in its production.

The final step is to feed the simulation into the reconstruction software in the same way as real data.

\subsection{Neutrino Events in the MINOS Detectors}

\subsubsection{Event Topologies}

Neutrino events from the NuMI beam break down into four broad categories: $\mathrm{CC}$ muon neutrinos $\left(\mathrm{CC} \nu_{\mu}\right), \mathrm{CC}$ muon antineutrinos $\left(\mathrm{CC} \bar{\nu}_{\mu}\right)$, CC electron neutrinos/antineutrinos $\left(\mathrm{CC} \nu_{\mathrm{e}}\right)$, and $\mathrm{NC}$ interactions. $\mathrm{CC} \nu_{\tau}$ and $\bar{\nu}_{\tau}$ events do also occur, albeit rarely, however they have an almost irreducible background from $\mathrm{NC}$ interactions in the MINOS detectors.

A typical $\mathrm{CC} \nu_{\mu}$ or $\mathrm{CC} \bar{\nu}_{\mu}$ event will have both a long, curved, muon track and a hadronic shower starting at the interaction vertex. The two events can be distinguished from one another by the electric charge of the muon produced, $\mu^{+}$for $\bar{\nu}_{\mu}$ or $\mu^{-}$for $\nu_{\mu}$, which can be determined by the curvature of the muon track in the MINOS detector's magnetic field. In normal neutrino mode running the detector fields are tuned to focus $\mu^{-}$inwards and the opposite for antineutrino running such that the signal event muon tracks always have a better chance of stopping inside detector and the more accurate range momentum can be used (discussed 
in section 3.8.2).

Both $\mathrm{CC} \nu_{e}$ and $\mathrm{NC}$ events produce interactions without muon tracks, though occasionaly with very short misreconstructed tracks. $\mathrm{CC} \nu_{e}$ events can be distinguished from $\mathrm{NC}$ events by their compact electromagnetic showers as opposed to $\mathrm{NC}$ events which contain diffuse hadronic showers. Whilst for the purpose of this thesis both these classes of events are background, members of the MINOS collaboration have used both the $\mathrm{NC}$, and $\mathrm{CC} \nu_{e}$ samples. Figure 3.14 shows example event topologies in the MINOS detectors for $\mathrm{CC} \nu_{\mu}, \mathrm{CC} \nu_{\mathrm{e}}$, and $N C$ interactions.
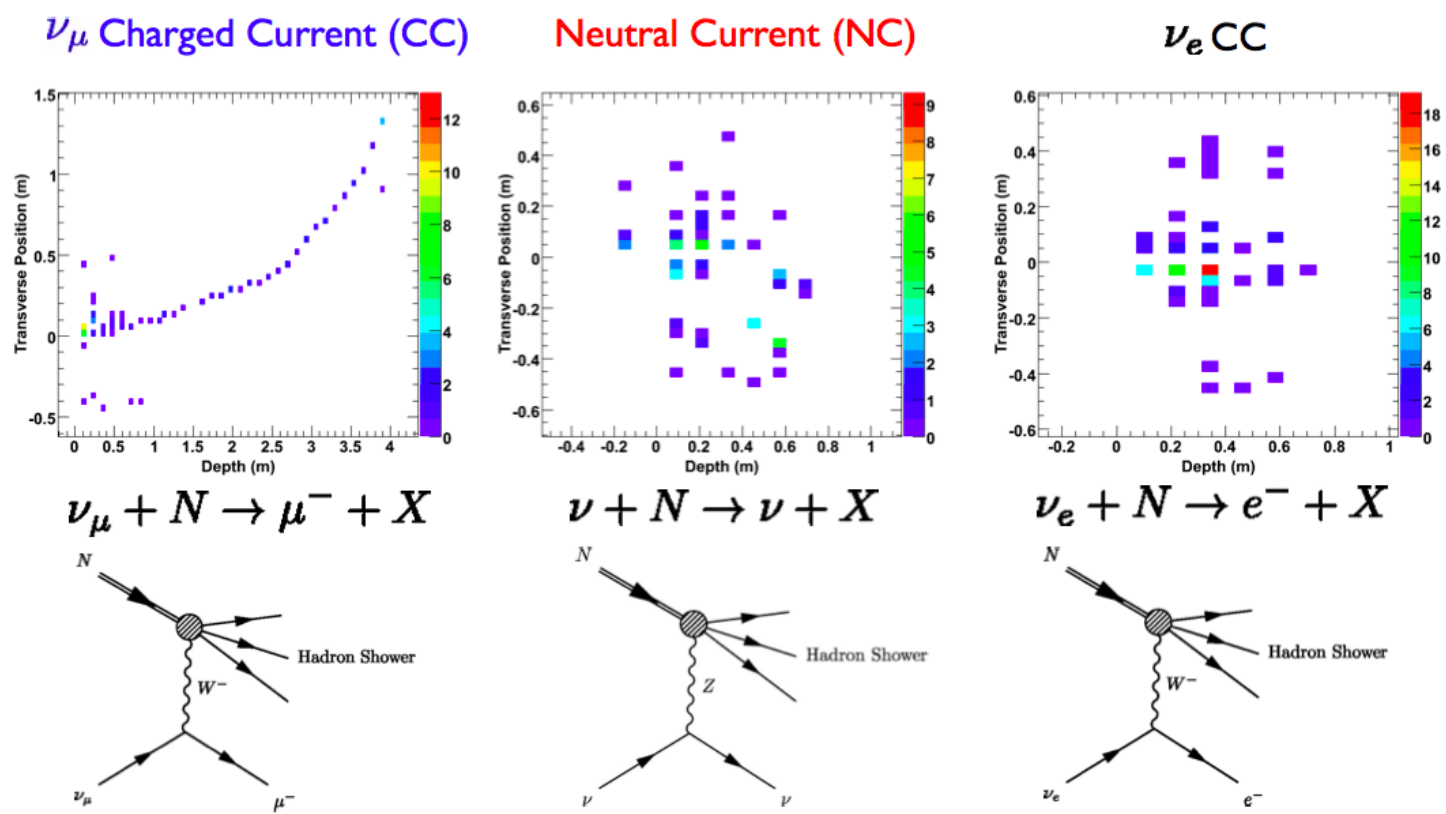

Figure 3.14: On the left a $\mathrm{CC} \nu_{\mu}$ interaction with a clear curving muon track which allows for both $\mathrm{CC} / N C$ identification and distinction between $\mathrm{CC} \nu_{\mu}$ and $\mathrm{CC} \bar{\nu}_{\mu}$. In the middle a NC interaction characterised by the lack of a track. On the right a $\mathrm{CC} \nu_{\mathrm{e}}$ interaction with a very short $e^{-}$track making it difficult to separate from the $N C$ sample. 


\subsubsection{Reconstruction}

The raw data from the detectors needs to be converted into the tracks and showers mentioned above so that not only can the type of interaction be distinguished but so that important information such as the energy of the parent neutrino can be measured as accurately as possible.

\section{Tracks}

For each individual event a track finder searchers for small "track like" segments, characterised by several hits in an approximate line across several planes. The track finder joins these segments together to form a "seed track" which is then fit using a multi-pass Kalman filter[91]. The filter moves up and down the track trying to estimate the momentum of the underlying muon at each point along the track whilst taking into account the effects of noise, multiple scattering, and expected curvature in the magnetic field. The filter decides on which of the components of the seed track form the reconstructed track.

After two passes of the filter properties of the fitted track such as its curvature, or electric charge to momentum ratio, and the uncertainty on that measurement are recorded. The curvature of the track is directly proportional to the ratio of the electric charge to its momentum and at $3 \mathrm{GeV}$, the low energy NuMI beam peak, the resolution is $11 \%[79]$.

For tracks ending in the detector (though not in the unistrumented coil hole) there is a second, more accurate, measurement of the momentum using the range of the track through layers of steel and plastic. At $3 \mathrm{GeV}$, the low energy NuMI beam peak, the resolution is $4.6 \%[79]$. The resolution power comes from the fact 
that the energy loss in $10 \mathrm{MeV}$ to $10 \mathrm{GeV}$ muons (the region of interest for oscillations) is well described by ionization and hence the Bethe-Bloch equation 92 . The energy is measured by swimming a muon backwards along the track in a GEANT3 simulation and summing the energy that would have been lost in each plane of the detector.

Confirmation of the Bethe-Bloch equation as tabulated by Groom [93] (with matter specific density effects specified by Sternheimer) comes from CalDet. When modified with the Groom tabulation the GEANT3 simulation achieved better than $2 \%[79]$ agreement between data and MC. The detector must be well measured and modelled for this technique to work, particularly the steel planes which account for $95 \%$ of the energy loss and are nominaly 1.46 radiation lengths thick. As already mentioned in this thesis (see section 3.3.2) the steel planes have been measured to an accuracy of $0.3 \%$ in density of and an accuracy in thickness of $0.1 \%$ at the Near and $0.2 \%$ at the Far in thickness.

\section{Showers}

After track identification all the remaining hits which are in proximity to one another are grouped into showers. Any hits which are part of a track but with more contained energy than a muon would have deposited have the track-portion of the energy subtracted and the remainder included in the shower. As the MINOS detectors are too coarse to distinguish the component particles reliably the energy of the shower was originally reconstructed calorimetricaly, that is an estimate based on the total energy deposited by the constituent hits.

An absolute shower energy scale is calibrated by well known test beams fired 
at the CalDet detector. Electron shower data agrees with the GEANT3 simulation to less than $2 \%$ and hadronic shower measurements showed agreement of $6 \%$.

The final calorimetric hadronic and electromagnetic shower resolutions are thus well modelled by the simulation and the resolution is parametrized as $56 \% / \sqrt{E}+$ $2 \%$ for hadrons and $21.4 \% / \sqrt{E}+4 \%$ for electrons, where $E$ is the particle energy in $\mathrm{GeV}$.

Calorimetric measurement of shower energy does however suffer from an inability to perfectly address energy loss at the calorimeter/spectrometer boundary, detector dead regions, non-linear Detector response, and hadronic non-compensation. Improvements were found by moving to a "k nearest neighbour" or "kNN" estimation of shower energy [94] which uses a multivariate analysis of a broader range of event level information to provide an estimate of shower energy. A training sample of Monte Carlo events passing the CC event selectors (discussed in section 4.1) were compared to test events by looking at " $D$ " distinct variables such that their separation is defined as:

$$
\Delta s^{2}=\sum_{i}^{d} \frac{\left(y_{i}-x_{i}\right)^{2}}{\sigma_{i}^{2}}
$$

where $s$ is their separation in this $\mathrm{kNN}$ space, $d$ is the number of distinct event variable examined, $y$ is the test event and $x$ is a member of the training sample. The kNN energy estimation is then the mean true energy of the kth nearest MC neighbours of our test event.

Three input variables or "figures of merit" were chosen, the calorimetric energy in the first two showers, deweighted (track energy removed) energy within $1 \mathrm{~m}$ of the track vertex, and the number of planes in the primary shower. An optimisation of 400 nearest neighbours was chosen based on an optimisation of improvement 
in resolution of neutrino mixing parameters. Far and Near MC for every MINOS run configuration each has a distinct training sample to account for detector and beam differences.

This kNN energy estimation is calibrated to an absolute shower energy by a simple ratio of kNN reconstructed and true shower energy (the estimated energy of reconstructed $\mathrm{MC}$ events and the true energy as defined in the $\mathrm{MC}$ ) for a large MC sample. At the lowest energies seen at MINOS this method provides a $40 \%$ increase in shower energy resolution. Figure 3.15 shows the difference between $\mathrm{kNN}$ and calorimetric energy resolution at a variety of energies.

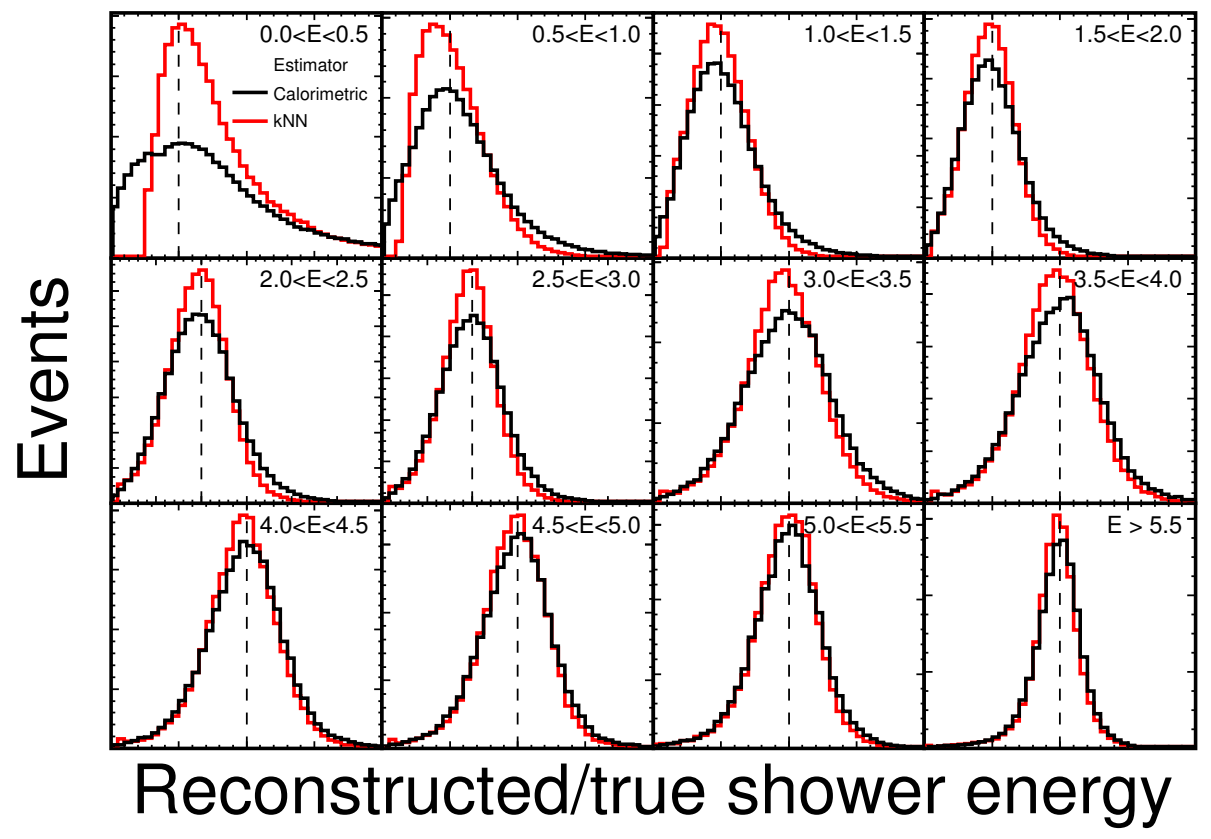

Figure 3.15: Distribution of $E_{\text {reco }} / E_{\text {true }}$ for different ranges of true shower energy. The standard reconstruction is in black, and the kNN energy estimate in red. The dotted lines mark $E_{\text {reco }}=E_{\text {true }}$. The $\mathrm{kNN}$ peak is sharper than standard reconstruction at low energies. Figure taken from [94] 


\section{Chapter 4}

\section{Studies of Muon Neutrino}

\section{Disappearance through \\ Oscillations at MINOS}

Every study of muon neutrino disappearance in the NuMI beam using the MINOS detectors, at its core, relies on the comparison of an observed spectrum of muon neutrino events at the MINOS Far Detector with a predicted spectrum based on the measurement of the NuMI beam at the Near Detector. In the simple two flavour oscillation approximation of muon neutrino disappearance at MINOS we have:

$$
P\left(\nu_{\mu} \rightarrow \nu_{\mu}\right) \approx 1-\sin ^{2}\left(2 \theta_{23}\right) \sin ^{2}\left(\frac{1.27 \Delta m_{a t m}^{2} L}{E}\right)
$$

Therefore the energy dependant depletion can then be fitted to atmospheric neutrino oscillations in order to measure $\Delta\left|m_{\text {atm }}^{2}\right|$ and $\sin ^{2}\left(2 \theta_{23}\right)$, with the depth of the depletion related to $\sin ^{2}\left(2 \theta_{23}\right)$ and the position of the dip in the spectrum related to $\Delta\left|m_{\text {atm }}^{2}\right|$. This is shown in Figure 4.1 .

However, in order to get to this final comparison there are a number of key 

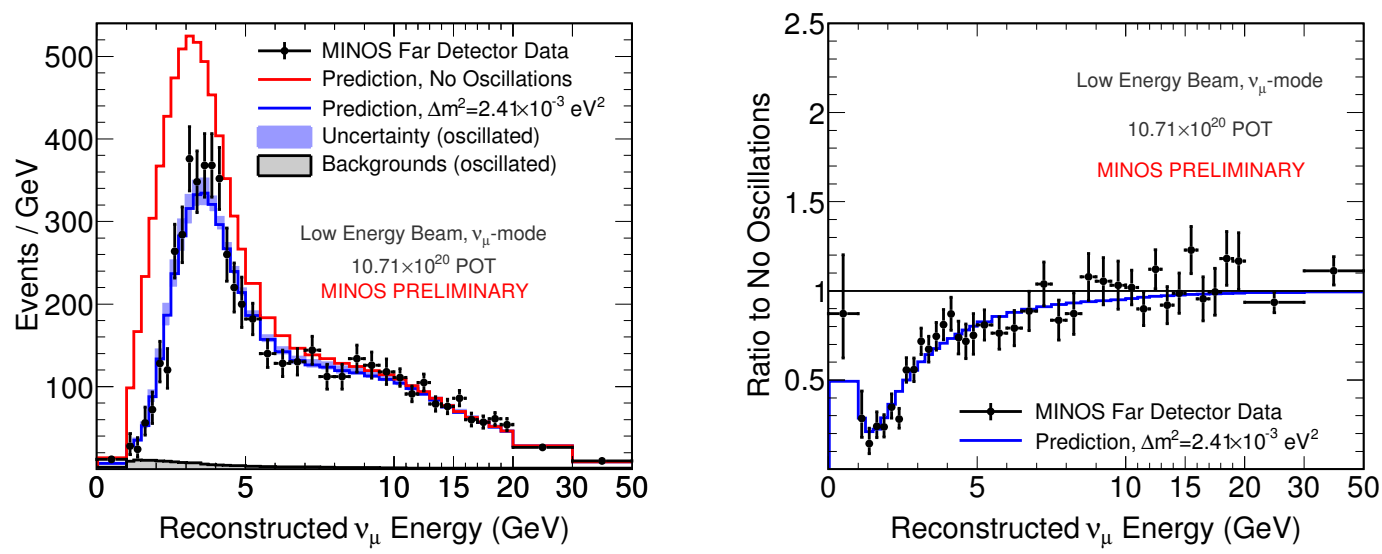

Figure 4.1: Left, Far Detector $\nu_{\mu}$ data and predictions for the no oscillations hypothesis (red histogram) and with the best-fit oscillation parameters from the best fit to the oscillations hypothesis (blue histogram). The band around the oscillated prediction represents the total systematic uncertainty described in section 4.3.1. Total background in the oscillated prediction is also displayed (gray shaded histogram). Right, ratio of Far Detector $\nu_{\mu}$ data to a null oscillation prediction (black) and a ratio of a Far Detector best-fit to oscillation prediction to a null oscillation prediction (blue).

steps, which are outlined below.

\subsection{Event Selection}

There are three main backgrounds to a muon disappearance analysis, namely NC events, atmospheric muon neutrinos, and "wrong sign" charge current events (events with their electric charge incorrectly reconstructed). NCs form a background to this analysis as they only contain information about the total neutrino flux rather than the muon neutrino event rate. Atmospheric neutrinos represent a potential source of muon neutrinos distinct from those in the muon beam and hence are not useful for a beam disappearance analysis. Wrong sign events become an important background if there is an attempt to measure only muon neutrino or muon antineutrino events where one only wants to select events with the correct lepton charge and hence correct electric charge. 
In addition to selection criteria to optimise signal purity and efficiency there are a number of data quality selection criteria which seek to make sure that only well understood NuMI/detector run times are used and that poorly reconstructed events do not enter the final sample.

The selection criteria used to acquire a highly quality sample of NuMI beam muon neutrino events at the Near and Far MINOS detectors are detailed here. Figure 4.2 shows the performance of the current FHC selection in the MINOS Near and Far Detectors.

\subsubsection{Preselection}

Preselection requires events to have good beam quality, good coil quality and the detector has to be operating properly. Good here means that they are as intended during that run period. This ensures the same criteria are applied to event selection that are applied at counting of protons on target (PoT). Light injection events used in calibration are cut out. We require the coil direction to be appropriate to the run type, i.e., the detector field focuses negative muon tracks in FHC or neutrino beam mode and the detector field focuses positive muon tracks in $\mathrm{RHC}$ or antineutrino beam mode. We reject any events where the error on the muon tracks reconstructed charge/momentum ratio, $\sigma(\mathrm{q} / \mathrm{p})$, equals $1 \times 10^{-4}$, which is an error code that indicates a failure mode of the Kalman filter and hence a poorly reconstructed event.

In order to remove neutrino events that don't come from the NuMI beam events must be recorded in time with a beam spill, in a standard 2 to $12 \mu$ s spill window. Additionally, we remove events with $\cos (\theta)<0.6$ between the muon track 

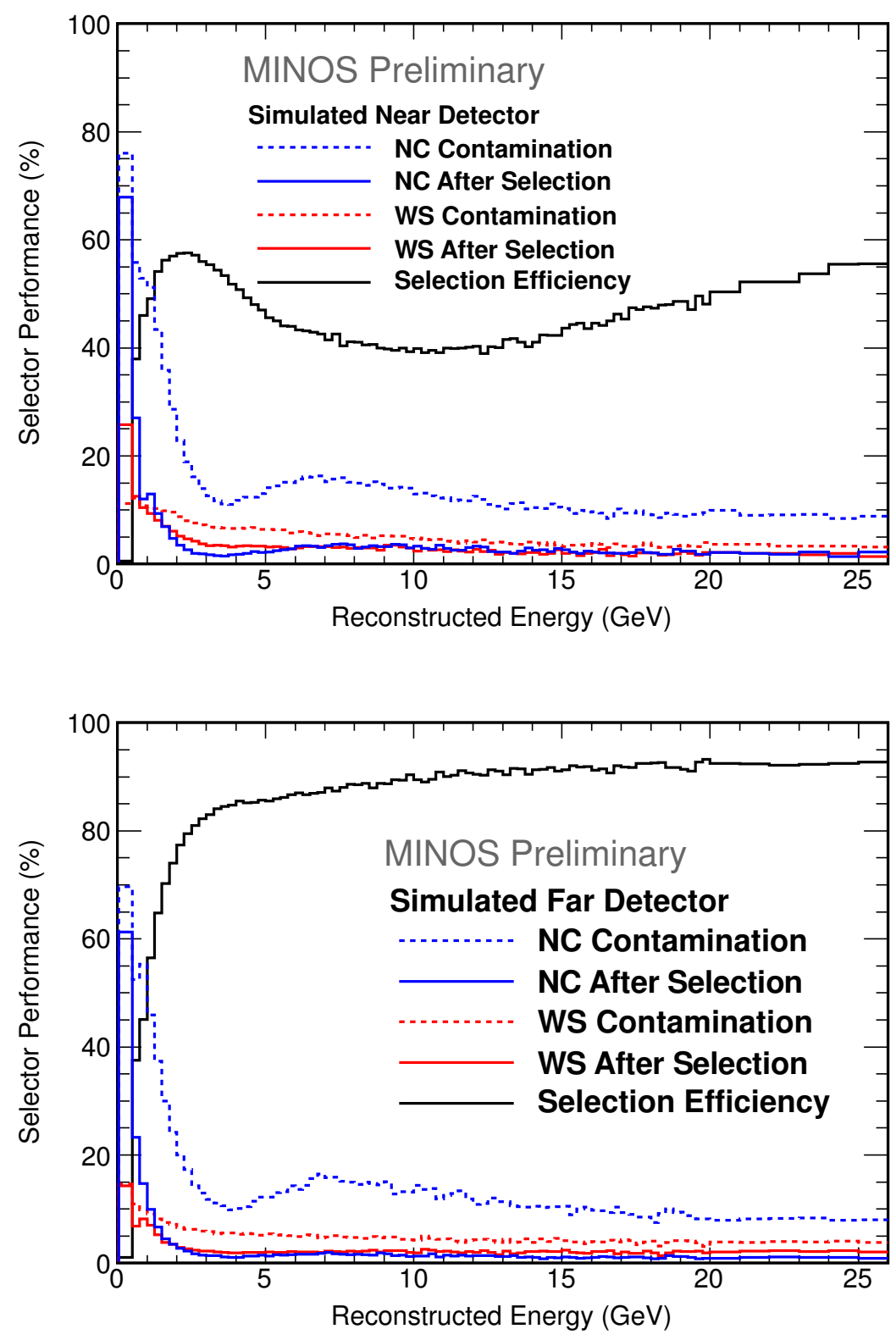

Figure 4.2: Performance of the current FHC selection in the Near (top) and Far (bottom) Detector, which is essentially a CC selection. The dashed lines show the contamination before selection and the solid show efficiency and contamination after selection. Neutral Current (NC) and Wrong charge Sign (WS) backgrounds are shown seperaretly. 

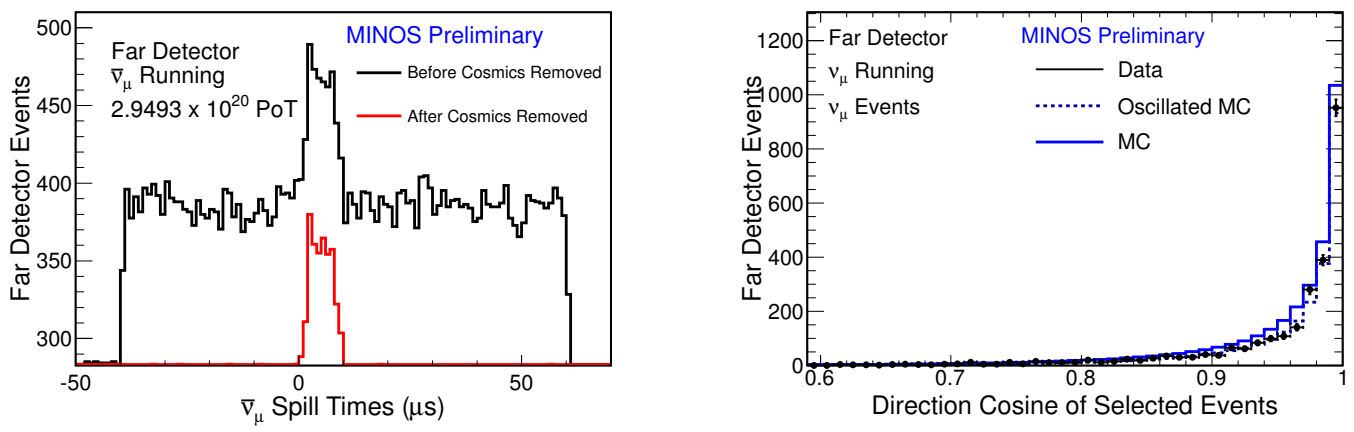

Figure 4.3: Left, the event spill timing plot before (black) and after (red) the cosmic background has been removed by preselection selection criteria. Right, cosine of the angle between the muon track and beam direction of $\nu_{\mu}$ events in the Far Detector. The dashed colored histogram represents the Monte Carlo expectation with the best fit oscillation parameters, the solid colored histogram represent the no oscillations expectation and black points represent data. MC is PoT normalized to data. Preselection removes events with $\theta<0.6$ to reduce background from cosmic rays.

and the beam line to further reduce cosmic and atmospheric backgrounds. The cosmic background selection criteria are shown in Figure 4.3 .

\section{Near Detector}

In the Near Detector we include an additional selection criteria to remove events which end near the coil hole. These coil hole events are very often very poorly reconstructed as the curvature of muon tracks is far more dramatic than events in the rest of the detector. The selection criteria is not required at the Far Detector where the fiducial volume is far larger, such that most of the tracks which end near the coil hole have tracks extending outside of the coil hole itself, thus allowing for better reconstruction.

The selection criteria removes tracks which end on the far side of the coil ( $\mathrm{x}$ $<0$, where $\mathrm{x}$ is the $\mathrm{x}$ horizantally orientated plane and 0 at the centre of the detector) or within $60 \mathrm{~cm}$ of the centre of the ND coil hole. They improve on the 

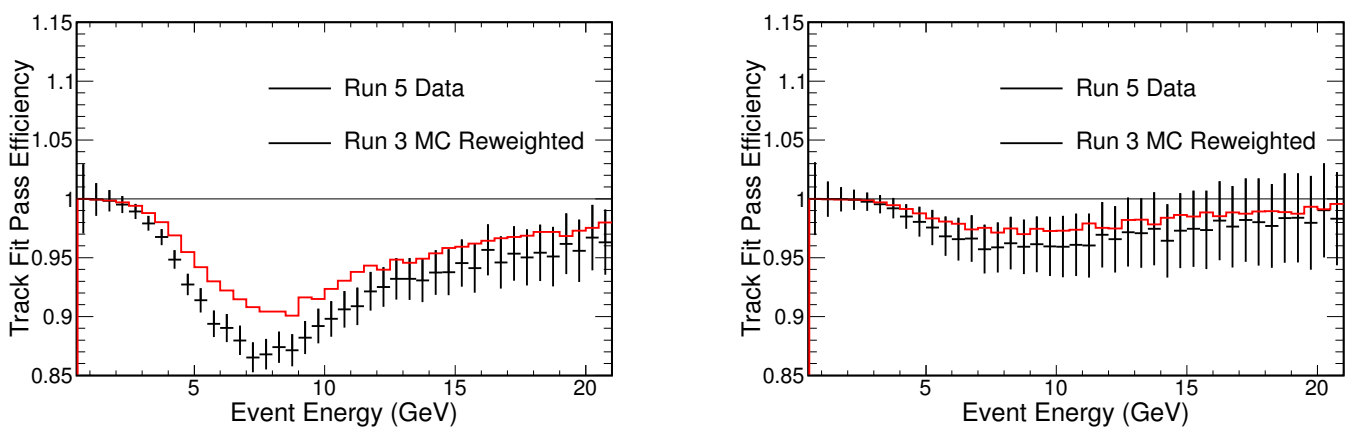

Figure 4.4: Track Fit pass efficiency of selected $\nu_{\mu}$ events in the Near Detector. The plot on the left does not have the new ND selection criteria, whilst the plot on the right does.

quality of our selected events by cutting out those which end near the coil hole of our Near Detectors focusing magnet and hence are badly reconstructed. This is most clearly seen in Figure 4.4, which not only shows better data/MC agreement with the new Near Detector selection criteria, but also shows that the ratio of selected events which pass our Track Fitter increases. Figure 4.5 shows how the muon track x position distribution is shaped by this selection criteria.

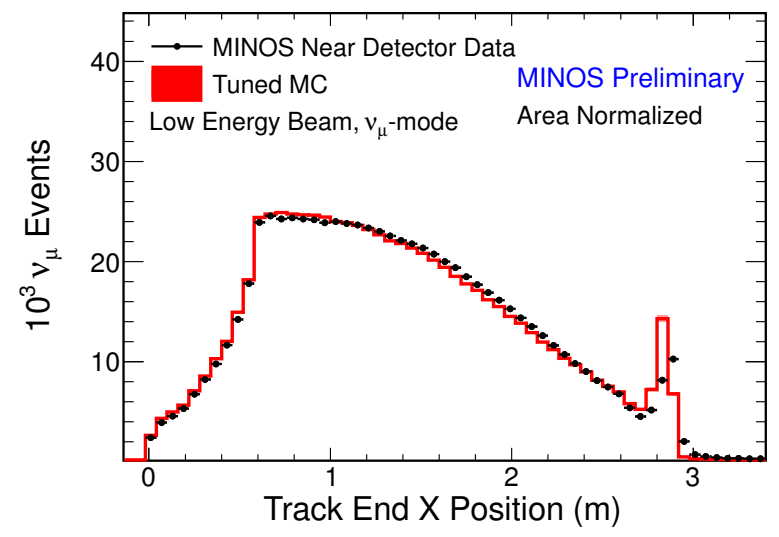

Figure 4.5: Near Detector track end X position distribution. The red histogram represents the Monte Carlo expectation with systematic uncertainties and black points represent data. The distribution shows the effect of the track end radius selection criteria at $0.6 \mathrm{~m}$. 
The Kalman fitter fails events which it was unable to reconstruct. Without the ND selection criteria our track fitter pass rate is $95 \%$, with the new ND selection criteria we achieve a $99 \%$ pass rate. Similarly, with the new ND selection criteria our data and MC agree within $2 \%$ at all energies, compared to $5 \%$ without. The better data/mc agreement and track fit pass rate combine to show that with the new selection criteria we are left with a cleaner ND data sample free of an unquantified reconstruction systematic.

\section{Far Detector}

In our Far Detector, if two or more events overlap within the time resolution of our Far Detector electronics, we only take an event if it has more than $75 \%$ of the total pulse height in that snarl, so as to reject falsely reconstructed events which have been separated from larger events. This selection criteria is unique to the Far Detector as it is a direct result of its different, slower, readout electronics which make resolution of two events very close in time problematic.

\subsubsection{Analysis Selection}

\section{Neural Current / Charge Current Separation}

$\mathrm{NC}$ and Charge Current events are separated using one of two " $\mathrm{k}$ nearest neighbour" or "kNN" algorithms. These kNN algorithms uses a number of variables related to the distinct features of a muon track associated with any true CC event. A Monte Carlo training set with two known classes of events is created, one with a muon track and one without. Events are then classed as being track-like or non-track-like by comparison to this training set using the $\mathrm{k}^{\text {th }}$ nearest neighbours in a Euclidean space defined by:

$$
D^{2}=\sum_{i}^{d}\left(X_{i}^{T} X_{i}^{Q}\right)
$$


where $D$ is the Euclidean distance, $d$ is the number of variables, $X_{i}^{T}$ is an event variable from the training set, and $X_{i}^{Q}$ an event variable from the query set (the set of events we are attempting to classify). We then look at the k nearest neighbours in the space defined by $D$. The probability of our query even being a $\mathrm{CC}$ event is given by:

$$
\mu_{I D}=\frac{k_{\mu}}{k_{\mu}+k_{n o n-\mu}}=\frac{k_{\mu}}{k}
$$

where $k_{\mu}$ is the number of those neighbouring training set events which contain muons and $k_{n o n-\mu}$ is the number which do not contain muons. $k=80$ was chosen to give good separation between the outputs for CC and NC events. This formalism is used to construct two measures of $\mu_{I D}$, the RoID, which is applied at all energies, and the jmID which is applied instead in the 0-5 GeV region when performing a FHC analysis.

\section{roID Particle ID}

The roID $\mathrm{CC} / \mathrm{NC}$ separation parameter uses 4 discriminating variables, shown in Figure 4.6, which all represent quantised common characteristics of a muon track in our detector, these are:

The number of active planes in a track. Muon tracks tend to extend much further than NC showers. This variable is the number of planes associated with the track.

Transverse profile parameter. Muon tracks will tend to be cleaner than a shower, depositing only single hits on a given scintillator plane. We quantise how separated the reconstructed hit is from the rest of the event by looking at the ADC pulse height fraction associated with the track. This is highly correlated with the energy deposited in the transverse vicinity of the track profile. 

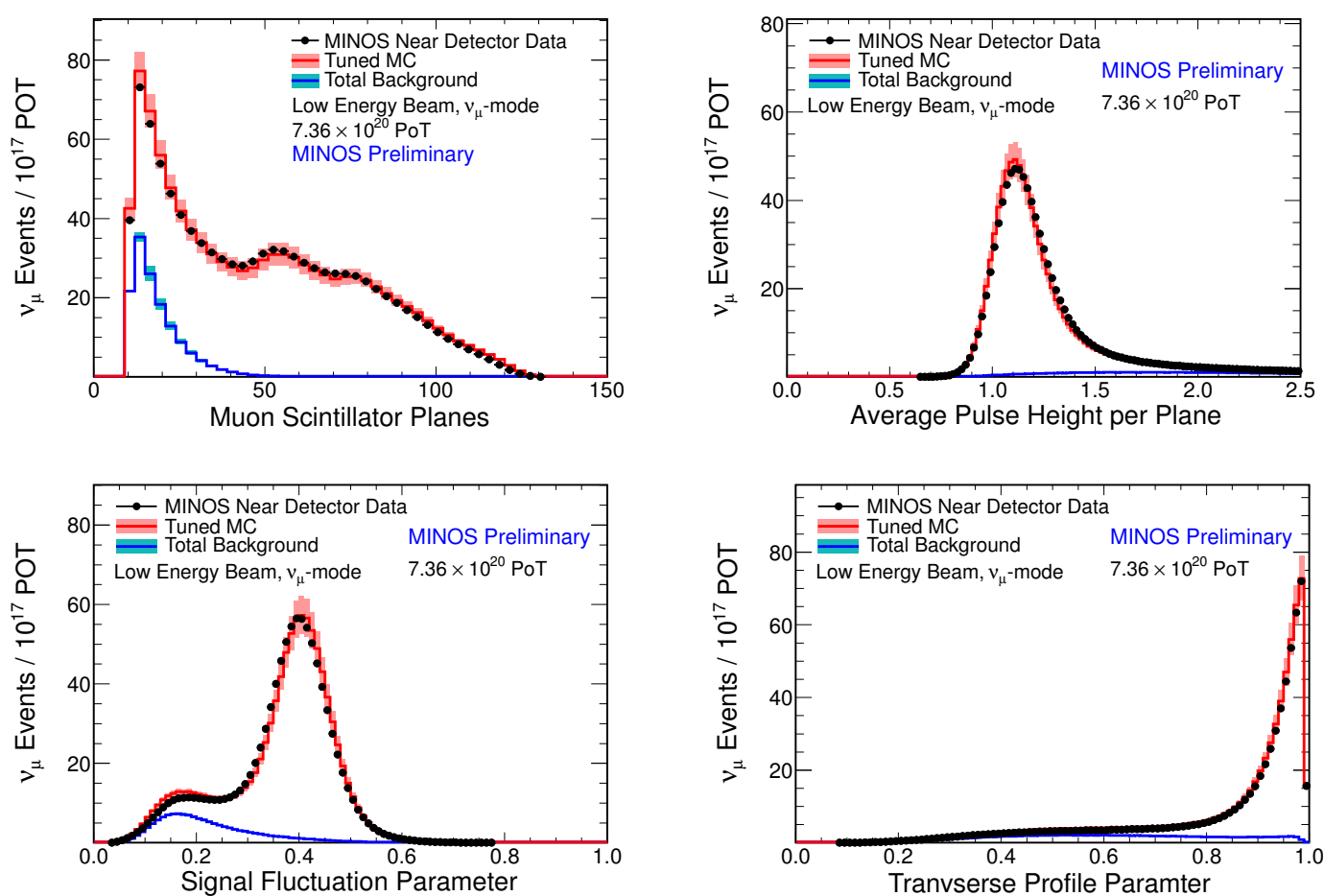

Figure 4.6: Distribution at the MINOS Near Detector for the four input variable to the RoID CC/NC "kNN" based discriminant. Shown is selected MC (red), data (black), and NC background in the selected MC (blue) with systematic uncertainties on the MC shown by the shaded region.

Average pulse height per plane in the track. Muon tracks are minimally ionising whereas hadronic showers will tend to have more energetic interactions. We quantise this by looking at the average energy deposited per plane away from the event vertex, which is highly correlated to $d E / d X$

Ratio of mean low pulse height to mean high pulse height. Muon tracks will deposit energy relatively smoothly when compared to hadron showers. We quantise this by looking at the ratio of the highest and lowest energy strips in the track. Only the first $30 \%$ of hits are used so as to avoid contamination from misreconstructed shower hits near the event vertex. 
The kNN discriminant they form is used to select CC events with a requirement that $\mu_{I D}>0.25$. The performance of this selector at the ND in the FHC disappearance analysis can be seen in Figure 4.7

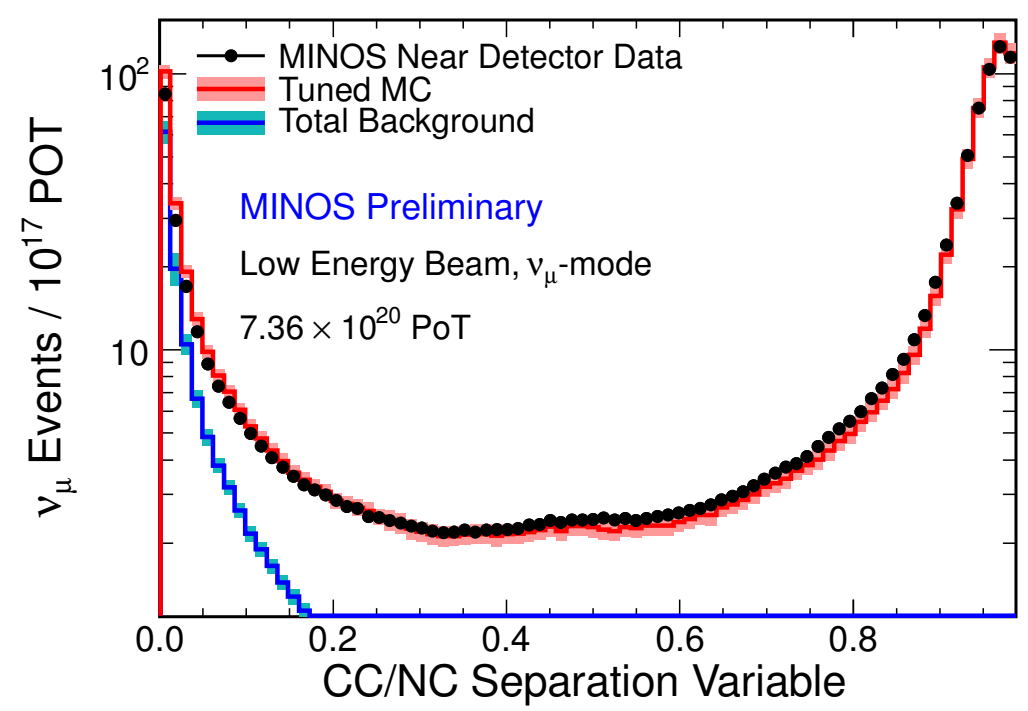

Figure 4.7: RoID CC/NC separation parameter of events passing all other selection selection criteria. The red histogram represents the Monte Carlo expectation with systematic uncertainties, the blue histogram represents the NC background with systematic uncertainties. Black points represent data. The selection criteria removes events with $\mathrm{PID}<0.3$.

\section{jmID Particle ID}

The jmID, is optimised for events in the $0-5 \mathrm{GeV}$ range where it is used in the FHC analysis. It represents an attempt to keep the efficiency of the sample as high as possible in the oscillation signal region. At lower energies the track variables used by RoID have reduced discriminating power as the detector's resolution makes distinguishing short muon tracks and NC showers more difficult. The jmID instead uses three parameters, shown in Figure 4.8, which are better suited for the identification of short muon tracks. These are: 
The number of active planes in a track. The same as described above for RoID.

Pulse height in the last five planes of the track. Unlike muons, hadronic showers will often terminate in a nuclear interaction, associated with a large energy deposit at the end of the track. We quantise this by looking at the mean pulse height in the last five planes of the track.

Degree of scattering. Muon well tend to have smooth, gently curving tracks, whereas hadronic tracks will undergo more scattering and plane-to-plane variation. We quantise scattering for the $U / Z$ and $V / Z$ views using the Pearson correction coefficient for the track. This coefficient is defined as:

$$
\rho=\frac{1}{N} \frac{\sum_{i}^{N} x_{i} z_{i}}{\sigma_{x} \sigma_{z}}
$$

where $x$ is the transverse position of the hit, $z$ is the longitudinal positions of the hit and $N$ is the total number of hits associated with the track. The final scattering variable is then:

$$
P=\frac{0.01}{1.01-\rho}
$$

The kNN discriminant they form is used to select CC events with a requirement that $\mu_{I D}>0.5$. The performance of this selector at the ND in the FHC disappearance analysis can be seen in Figure 4.9 

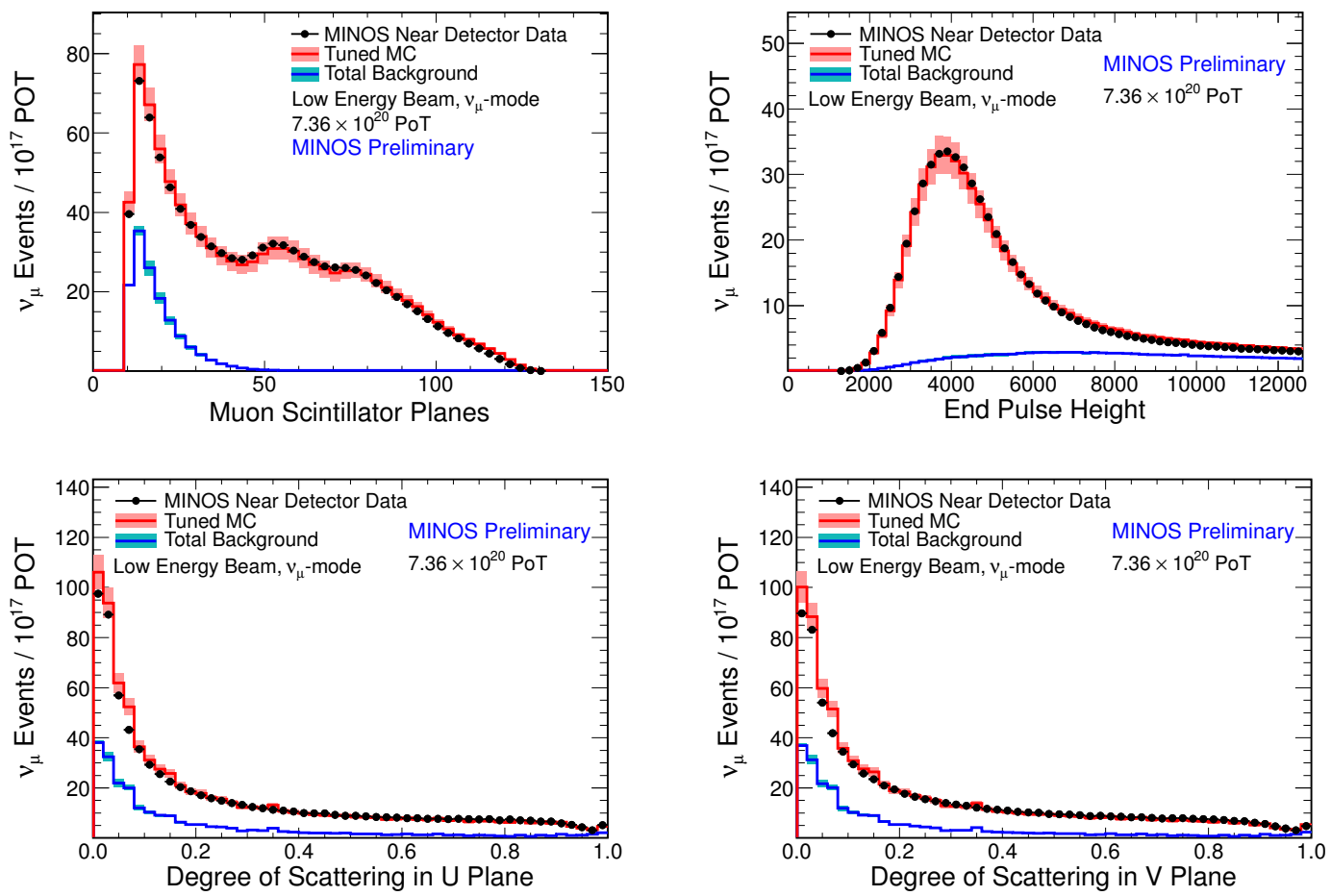

Figure 4.8: Distribution at the MINOS Near Detector for the four input variable to the jmID CC/NC kNN based discriminant. Shown is selected MC (red), data (black), and $\mathrm{NC}$ background in the selected MC (blue) with systematic uncertainties on the MC shown by the shaded region. 


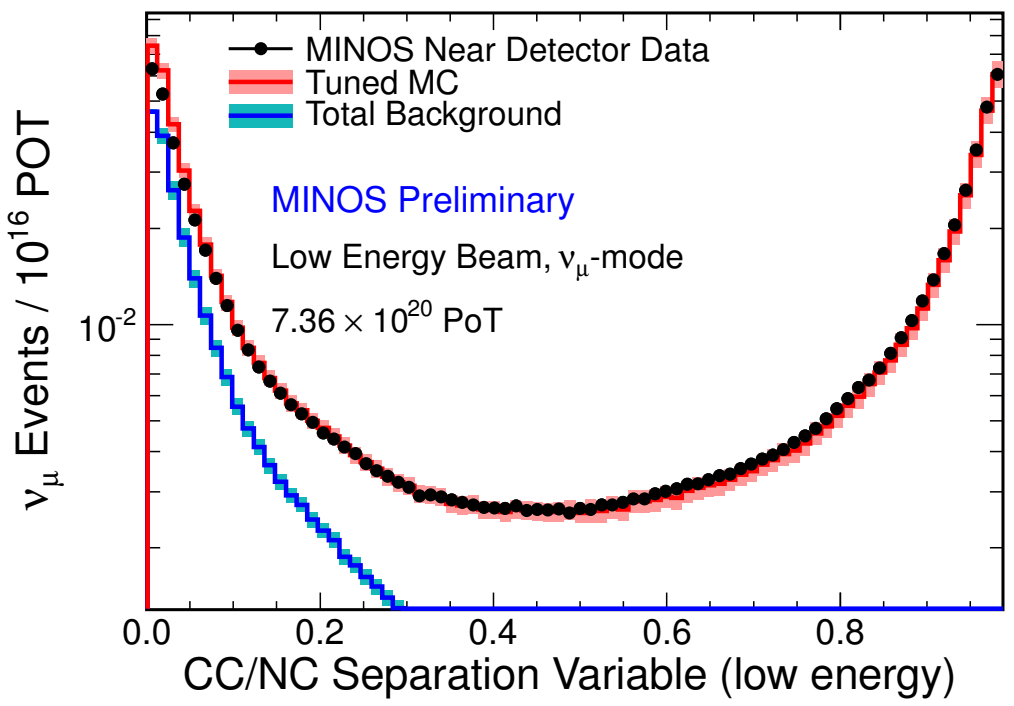

Figure 4.9: Low energy jmID $\mathrm{CC} / \mathrm{NC}$ separation parameter of events passing all other selection selection criteria. The red histogram represents the Monte Carlo expectation with systematic uncertainties, the blue histogram represents the NC background with systematic uncertainties. Black points represent data. The selection criteria removes events with PID $<0.5$.

\section{Charge Sign Separation}

$\nu_{\mu}$ and $\bar{\nu}_{\mu}$ separation is achieved by selecting based on the charge/momentum ratio, $q / p$, of the fitted muon track shown in figure 4.10 . Its curvature in the magnetised detectors is strongly indicative of the muons electric and hence leptonic charge. At low energies, where the track is very short, and high energies, were the track is very straight, the reconstructed $q / p$ may have a very large uncertainty $\sigma(q / p)$. This is less important for the FHC neutrino analysis which uses both neutrinos and antineutrinos in a 2 parameter fit assuming identical oscillations, however in the RHC and FHC antineutrino analyses which aim to provide a definitive measure of oscillation of $\bar{\nu}_{\mu}$ these wrong charge sign reconstructed events form a significant background. Consequentially the RHC antineutrino analysis 
requires the significance of the $q / p$ measured by the kalman fitter, $(q / p) / \sigma(q / p)$, be greater than 2.3. This selection criteria provides the best optimistion of efficiency and purity in the $\bar{\nu}_{\mu}$ sample, Figure 4.11 shows $(q / p) / \sigma(q / p)$ for selected antineutrino events.

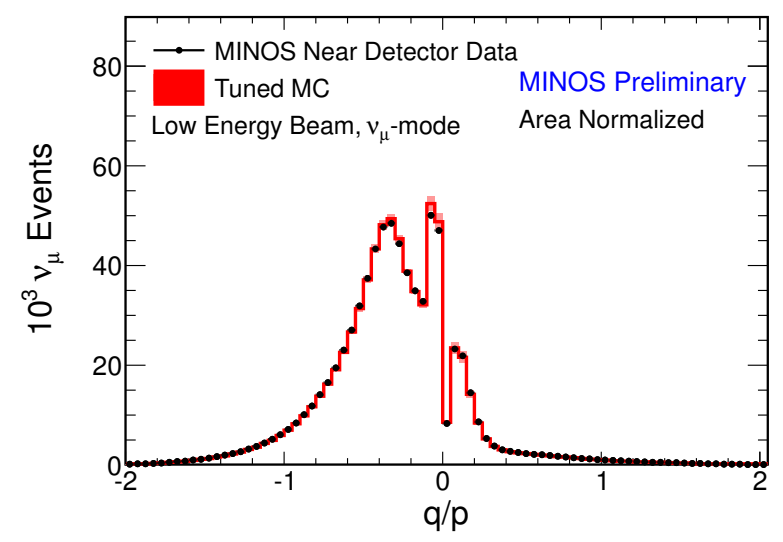

Figure 4.10: $(q / p)$ distribution of events passing all selection selection criteria in the Near Detector. The red curve represents MC expectation with the systematic uncertainty while the black dots represent data. 


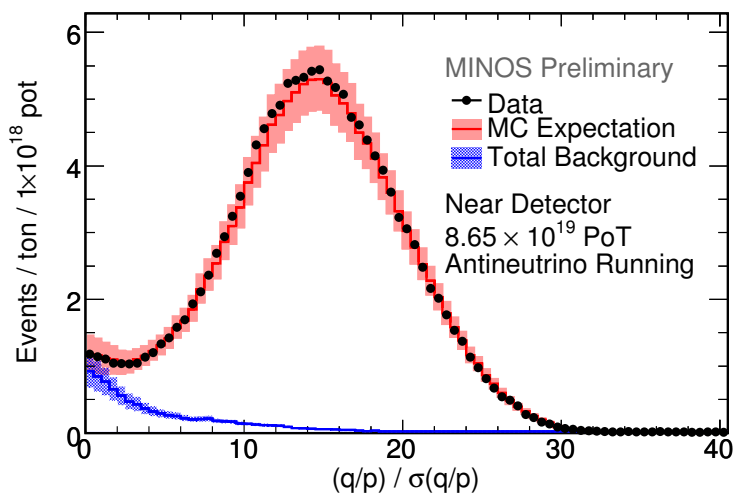

Figure 4.11: $(q / p) / \sigma(q / p)$ (track charge sign significance) distribution of selected antineutrino events in the Near Detector. The red curve represents MC expectation with the systematic uncertainty, the blue curve represents the $\mathrm{CC}$ and $\mathrm{NC}$ backgrounds with systematic errors and black dots represent data.

\subsection{Extrapolation}

The conversion of our measured Near Detector spectrum to a Far Detector prediction relies on three steps. First the Near Detector event spectrum must be converted into a measure of the neutrino flux through the Near Detector, then that flux must be extrapolated to the flux seen at the Far Detector, and finally that Far Detector flux must be converted back to a reconstructed event energy spectrum. Figure 4.12 shows the full process as a flow chart. 


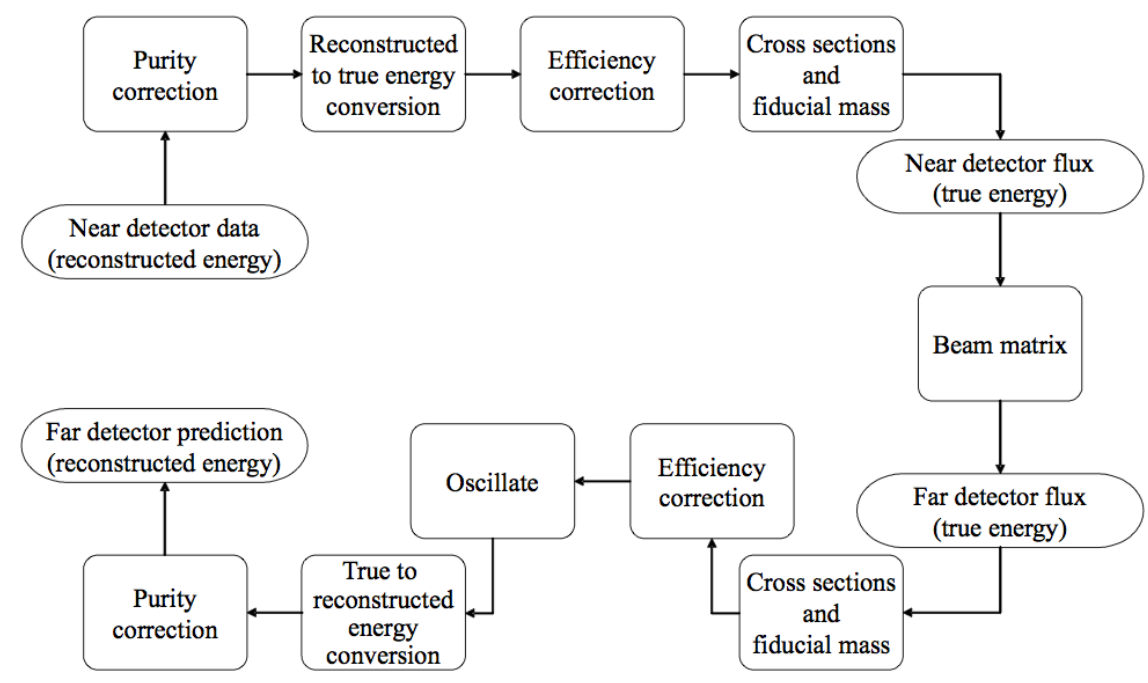

Figure 4.12: The steps in the extrapolation of a Near Detector measurement to a Far Detector prediction.

\subsubsection{Unfolding}

Unfolding the data to get the neutrino beam flux whilst accounting for finite detector effects can be broken down into several key steps. First, we correct for impurities in the measured spectrum using a prediction of purity as a function of reconstructed energy based on MC. Purity, $P$, for each bin $i$ of this histogram of Purity as a function of reconstructed energy is then defined as:

$$
P=\frac{N_{\text {sig }}}{N_{\text {tot }}}
$$

where $N_{\text {sig }}$ is the number of signal events selected, and $N_{\text {tot }}$ is the total number of selected events. This histogram, shown in Figure 4.13, is then multiplied with the measured Near Detector spectrum, bin-by-bin, to produce a reconstructed energy spectrum with impurities removed. 

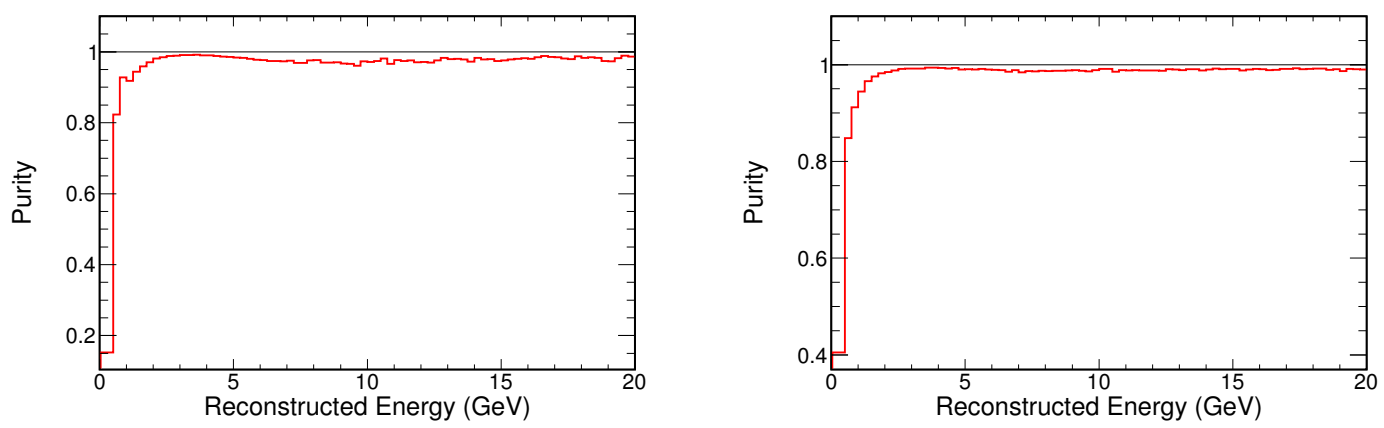

Figure 4.13: Purity as a function of reconstructed energy at the Near (left) and Far (right) MINOS detectors.
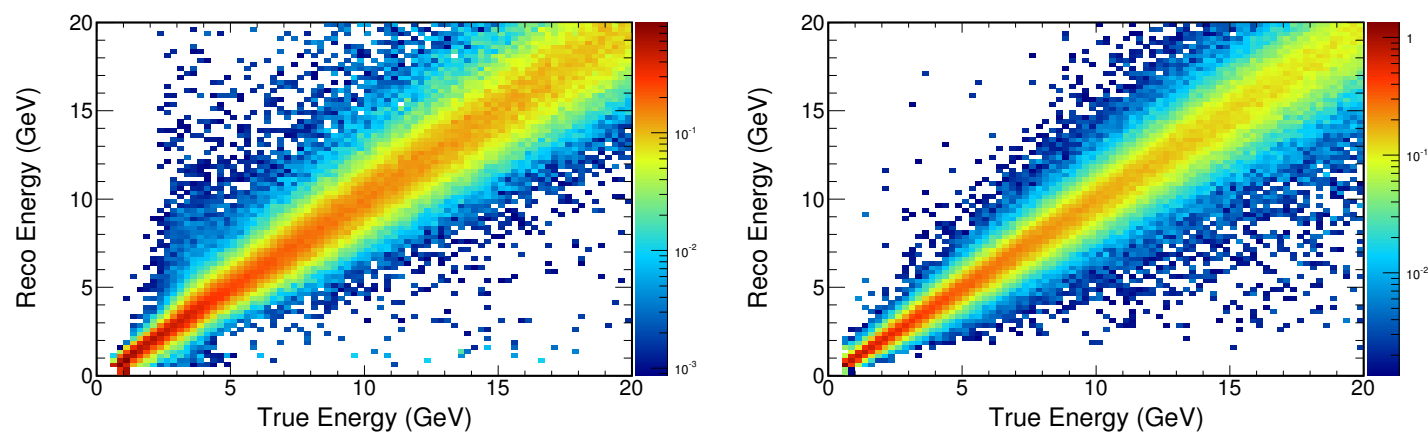

Figure 4.14: True vs. Reconstructed energy at the Near (left) and Far (right) MINOS detectors.

Next is the conversion from reconstructed to true energy, finite detector resolutions in event reconstruction mean that a given reconstructed event energy can correspond to a variety of potential true neutrino energies. We account for this by creating a $2 \mathrm{D}$ matrix of reconstructed to true energy based on a large sample of $\mathrm{MC}$ events reconstructed in the same way as the data. This matrix, shown in Figure 4.14, is normalized such that the array of possible true energies for each reconstructed energy sum to unity.

We then correct for efficiency in the measured spectrum using a prediction of efficiency as a function of true energy based on MC. Efficiency, $E$, for each bin $i$ 

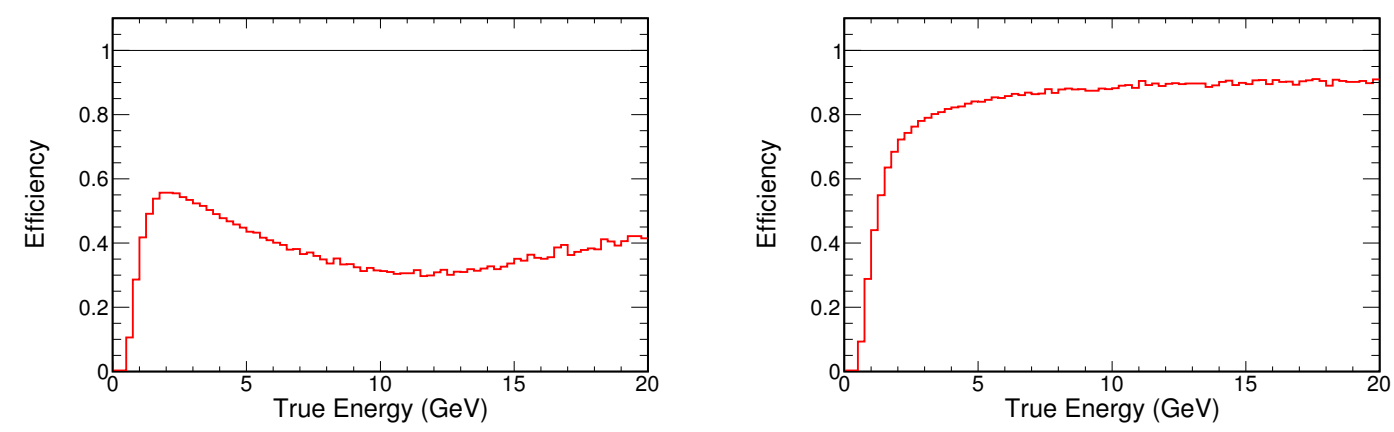

Figure 4.15: Efficiency as a function of true energy at the Near (left) and Far (right) MINOS detectors.

of this histogram of efficiency as a function of true energy is then defined as:

$$
E=\frac{N_{\text {sig }}}{N_{\text {fid }}}
$$

where $N_{\text {sig }}$ is the total number of signal events selected and $N_{f i d}$ is the total number of signal events in the fiducial volume. This histogram, shown in Figure 4.15, is then multiplied with the purity corrected Near Detector spectrum in true energy, bin-by-bin, to produce a true energy spectrum with inefficiencies removed.

Finally, we renormalise by the fiducial mass and cross sections at the Near Detector to get an approximation of the total neutrino flux passing through it. The flux passing through the detector, per proton on target, as a function of energy is then:

$$
F_{D}=\frac{T_{D}}{X m_{D} P_{D}}
$$

where $m_{D}$ is the detectors fiducial mass, $p_{D}$ is the number of protons on target corresponding to the exposure of the detector to the NuMI neutrino beam, $X$ is the $\mathrm{CC}$ cross section as a function of energy, and $T_{D}$ is the energy spectrum of true muon neutrinos which interact in the detector.

After the beam matrix, described in section 4.2.2, has been applied we perform 

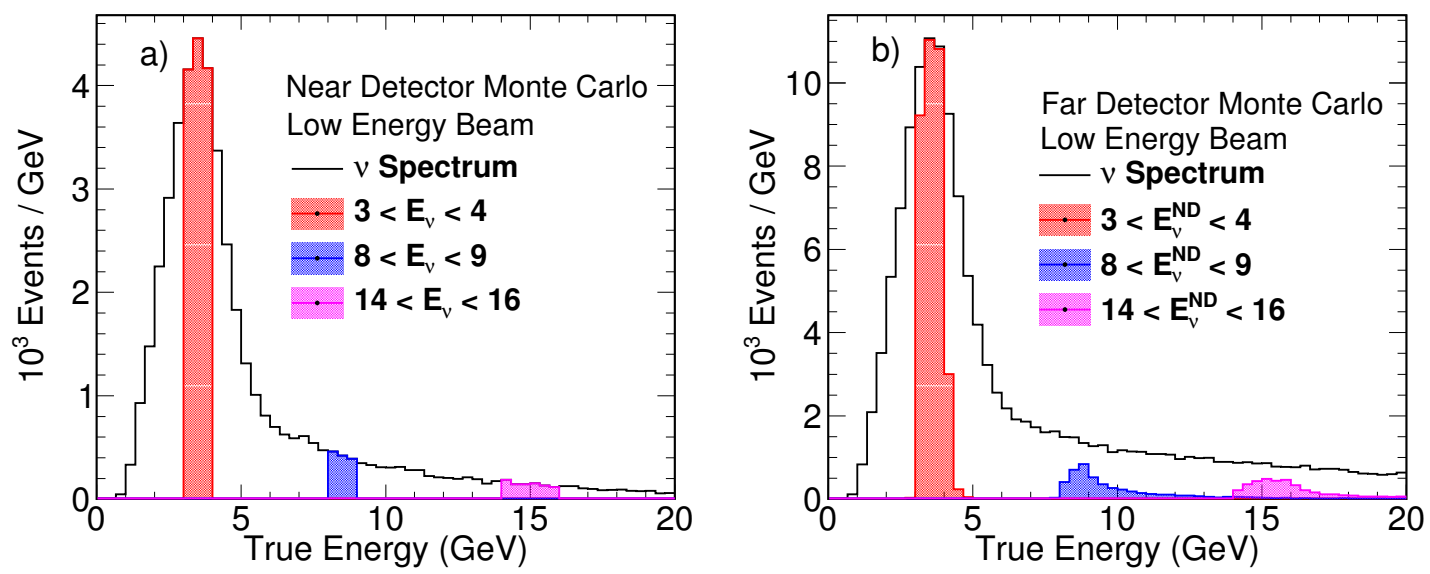

Figure 4.16: Example muon neutrino energy spectrum at the Near (left) and Far (right) MINOS Detectors. The coloured regions in each plot correspond to the same neutrino parent meson decay positions and momenta. Differences in geometric acceptance at the detectors leads to both the smearing of this highlighted regions and the differences between the two spectra.

these steps inverted where oscillations are being applied as a function of true energy just before the conversion from true to reconstructed energy.

\subsubsection{Beam Matrix}

The different angular acceptances between the detectors means that a given energy bin in the Near Detector spectrum can contribute to a range of bins at the Far Detector. As the Near Detector is much closer to the beam pipe than the Far Detector it represents a much larger solid angle to any decaying hadron. Neutrino decay from a Pion or Kaon is isotropic so the Near Detector will see more low energy, slightly off axis, pions than the Far Detector. Figure 4.16 shows the how each detector sees different contributions from the same neutrino parents.

The simulation of our flux contains detailed kinematic information about the neutrino parents, this means we can approximate the energy and probability a 
neutrino which will pass through either detector. This technique is explained in detail in section 7.2. We take advantage of this by calculating a weighted neutrino energy for each parent for a randomly selected Near Detector interaction vertex and a central interaction vertex in the Far Detector (which relative to NuMI is pointlike). This gives us a Near and Far Detector energy which is associated by a common neutrino parent and by scanning over a large set of $\mathrm{MC}$ we can construct a matrix relating every bin in energy at the Near Detector to a range of bins in the Far Detector. The final step is then to normalize each column to one Near Detector neutrino to produce Figure 4.17, which can be multiplied by a Near Detector flux to obtain a Far Detector Flux. This process is carried out for each possible NuMI run configuration such that we have specific beam matrices for any NuMI target configuration.

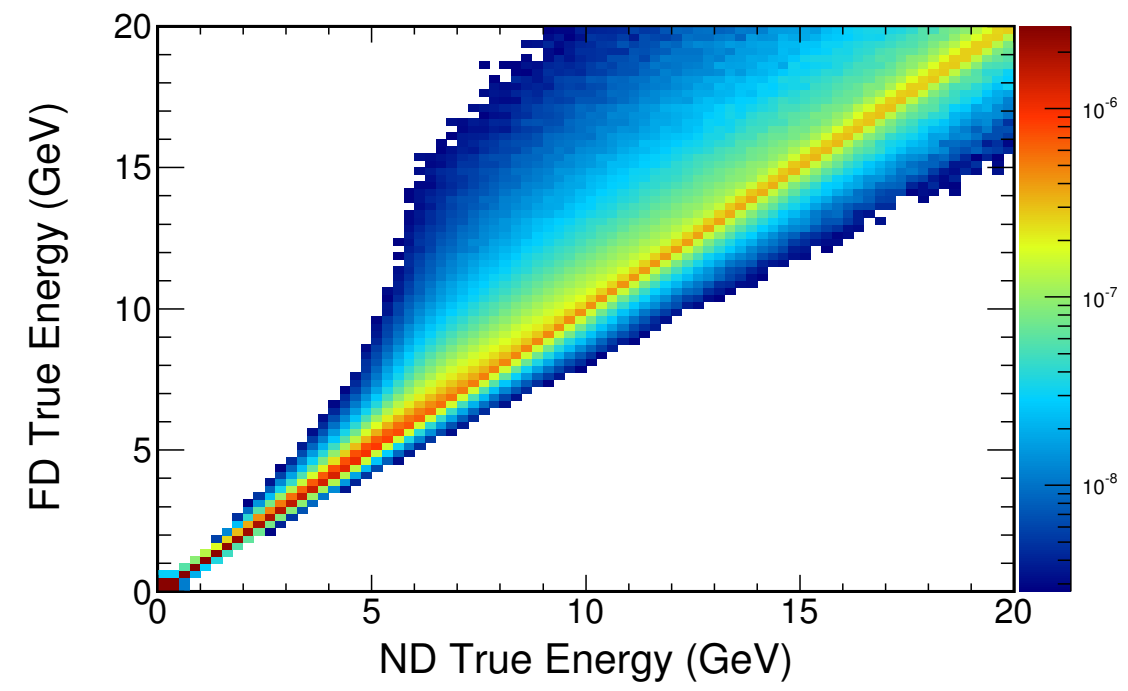

Figure 4.17: Beam matrix used to convert a neutrino Near Detector flux into a Far Detector flux. 


\subsection{Systematics}

The following are short summaries of the largest sources of systematic uncertainty, and their origin:

Normalisation: There is an uncertainty on the relative normalisation of the selected Near and Far Detector event samples. This uncertainty is dominated by differences in the reconstruction and selection efficiencies between the two detectors, as well as relative uncertainties on fiducial mass and live time. This is quantified by extensive hand scanning of $\mathrm{MC}$ and data from both detectors. A group of MINOS collaboration members where given overlapping subsets of MINOS Data and MC and asked to evaluate the event reconstruction of discrete events. The average disagreement bewteen the reconstruction and the hand scanner disagreement in Data and MC then provides a reconstruction normalisation uncertainty. Taking this into account we have a total normalisation uncertainty of $1.6 \%$.

Wrong Sign Background: Misidentified CC- $\nu_{\mu}$ events make up a significant background to our $\bar{\nu}_{\mu}$ sample. In order to quantify the uncertainty on this background we look at a subset of data and MC events with a large relative uncertainty on their electric charge (i.e., with $(q / p) / \sigma(q / p)>2.3$, where $q / p$ is the electric charge momentum ratio we use to identify charge and $\sigma(q / p)$ is the uncertainty on this measurement). This provides a sample enriched in this background, and therefore any discrepancies between the data and $\mathrm{MC}$ can be attributed to the $\mathrm{CC}-\nu_{\mu}$ background. The maximum size of the discrepancy we see is on the order of $30 \%$ and hence we use this as the uncertainty on the Wrong sign background.

NC Background: The uncertainty on the NC background is quantified by two 
studies. The first compares the data and MC energy spectra of all preselected events that would not pass our CC selection criteria (below a PID value of 0.3). Any data/MC discrepancies in this region are then attributed to the NC sample. The other is again a data/MC comparison, but in this case selected $\mathrm{CC}$ events where the muon has been removed to create a NC-like sample of events which is independent from the actual NC MC. The sum in quadrature of the discrepancies seen in the two independent studies is of order $20 \%$ and therefore this is used as the uncertainty on the $\mathrm{NC}$ background.

Shower Energy Scale: There are two different uncertainties on the shower energy scale [94]. A relative near to far mismodelling, and an absolute mismodelling. The relative mismodelling comes from uncertainties on the energy calibration of the two detectors, the Near Detector has an uncertainty of $1.9 \%$ dominated by a difference between the spill and cosmic MEU (muon energy unit) numbers in $\mathrm{MC}$ which is not seen in the data. The Far Detector has a 1.1\% uncertainty which is dominated by no single uncertainty.

The absolute uncertainty comes from a number of different sources. One is the uncertainty on the energy deposition of single hadrons in CalDet which is $5 \%$ summed in quadrature with a $2 \%$ uncertainty on the CalDet beam energy and a $1.4 \%$ on the CalDet stopping-muon calibration. Another is that cosmic MEU numbers in the Near and Far Detector are actually both consistently lower than the spill numbers in both detectors, by $0.9 \%$. Adding in quadrature this gives us a $5.7 \%$ uncertainty at all shower energies [95].

There is also a contribution from the uncertainties on the modelling of hadronic showers to the absolute shower energy uncertainty. This is fully explored in [96], 
where the uncertainty is given as a function of energy with an uncertainty of $8.32 \%$ in the $0-0.5 \mathrm{GeV}$ bin reducing to $\approx 3 \%$ at $10 \mathrm{GeV}$. The energy dependant form is used, summed with the flat $5.7 \%$ uncertainty to give:

$$
\sigma_{s h w}=6.6 \%+(3.5 \%) \times e^{-\frac{E_{r e c o}}{1.44 G e V}}
$$

where the uncertainty is taken as $100 \%$ correlated bin to bin.

Track Energy Scale: There is a $2 \%$ uncertainty on muon track energy from range, and the uncertainty on the energy from curvature is assessed by comparing range and curvature for stopping tracks and found to be 1\%. The uncertainties are taken to be fully correlated between the detectors.

Acceptance: The acceptance uncertainty covers the difference between acceptance of positive and negative charged tracks in the Near Detector. The uncertainty on this systematic is energy dependant, assessed by the difference in FD Prediction made with Near Detector data from RHC- (negative electric charge track focusing Near Detector magnet polarity) and RHC+ (positive electric charge track focusing Near Detector magnet polarity) runs. This was then applied to the Monte Carlo by using a ratio plot as a reference and shifting each Near Detector events weight by an amount proportional to the deviation from unity at that energy in the ratio.

Cross Sections: There are a number of cross section systematics stemming from large uncertainties in the modelling of different cross sections. These large uncertainties come from the paucity of neutrino cross section data available to the community at present. Uncertainties on cross sections related to coherent pion production, resonance production, quasi elastic collisions, and deep inelastic scattering are all accounted for (more information on each of these processes can 
be found in [59]). However because of the similiar designs of the MINOS Near and Far Detectors they are extremely correlated such that they largely cancel out in the final Far/Near ratio.

Flux Modelling: There is also a small uncertainty on the flux, it is based on uncertainties on hadron production, beam optics, target position, and material budget. However, it is fully correlated between the detectors which means the uncertainty on the final Near/Far Detector ratio is actually negligible.

\subsubsection{Evaluating the Impact Of Different Systematics}

In order to gauge the systematic uncertainties on the oscillation parameters measured in our disappearance analyses, high statistics "fake data" sets was used. Fake data sets are sets of MC which we treate as data, using truth information in the Far Detector MC to apply oscillations. They were generated from Far and Near Detector Monte Carlo events by applying shifts to each individual event for each systematic uncertainty separately and oscillations where applied to the Far Detector monte carlo using our best fit values to the mixing parameters.

Generated events were treated like real data and passed through the entire analysis chain before fitting for the oscillation parameters. The shifts of the best fit values of the oscillation parameters relative to the nominal case were then calculated.

The systematic shifts of the oscillation parameters are shown graphically in Figure 4.18. Shown in Figure 4.19 are the systematic shifts together with the statistical sensitivity contour. As can be see in Figure 4.19 the systematic uncertainty remains subdominant to our statistical uncertainty. 
A similar technique can be used to assess the systematic uncertainty on the number of events in each bin of energy for the Near Detector and Far Detector predicted spectra. Again systematically shifted Near and and Far Detector "fake data" sets are created. The fractional systematic error for any one systematic at the Near Detector is given by:

$$
\sigma_{N}=1-\frac{\text { Shifted ND Fake Data }}{\text { Nominal ND Fake Data }}
$$

and at the Far Detector prediction:

$$
\sigma_{\frac{F}{N}}=1-\frac{\text { Shifted FD Prediction/Shifted FD Fake Data }}{\text { Nominal FD Prediction/Nominal FD Fake Data }}
$$

The double ratio at the Far Detector accounts for cancellations between the Near and Far Detectors. Shifts are added in quadrature for every energy bin to obtain an energy dependant uncertainty shown in Figures 4.20. This band is not used in the fit but is included in the final result plots showing our best fit prediction and data. The related techinque for including our systematic uncertainty in the final fit is detailed in Section 4.4.1

\subsection{Fitting}

Once we have an extrapolated Far Detector predicted spectrum based on a measured Near Detector Spectrum and a measured Far Detector Spectrum we have the tools needed to fit for the neutrino mixing parameters. Fits are made using a binned log likelihood method with best fit oscillation parameters found at the minimum of the log-likelihood ratio. The figure of merit for goodness of fit is then the Poisson Deviance, which for a combination of $\Delta\left|m_{a t m}^{2}\right|$ and $\theta_{23}$ is:

$$
\chi^{2}=2 \sum_{i} e_{i}-o_{i}+o_{i} \ln \left(\frac{o_{i}}{e_{i}}\right)
$$



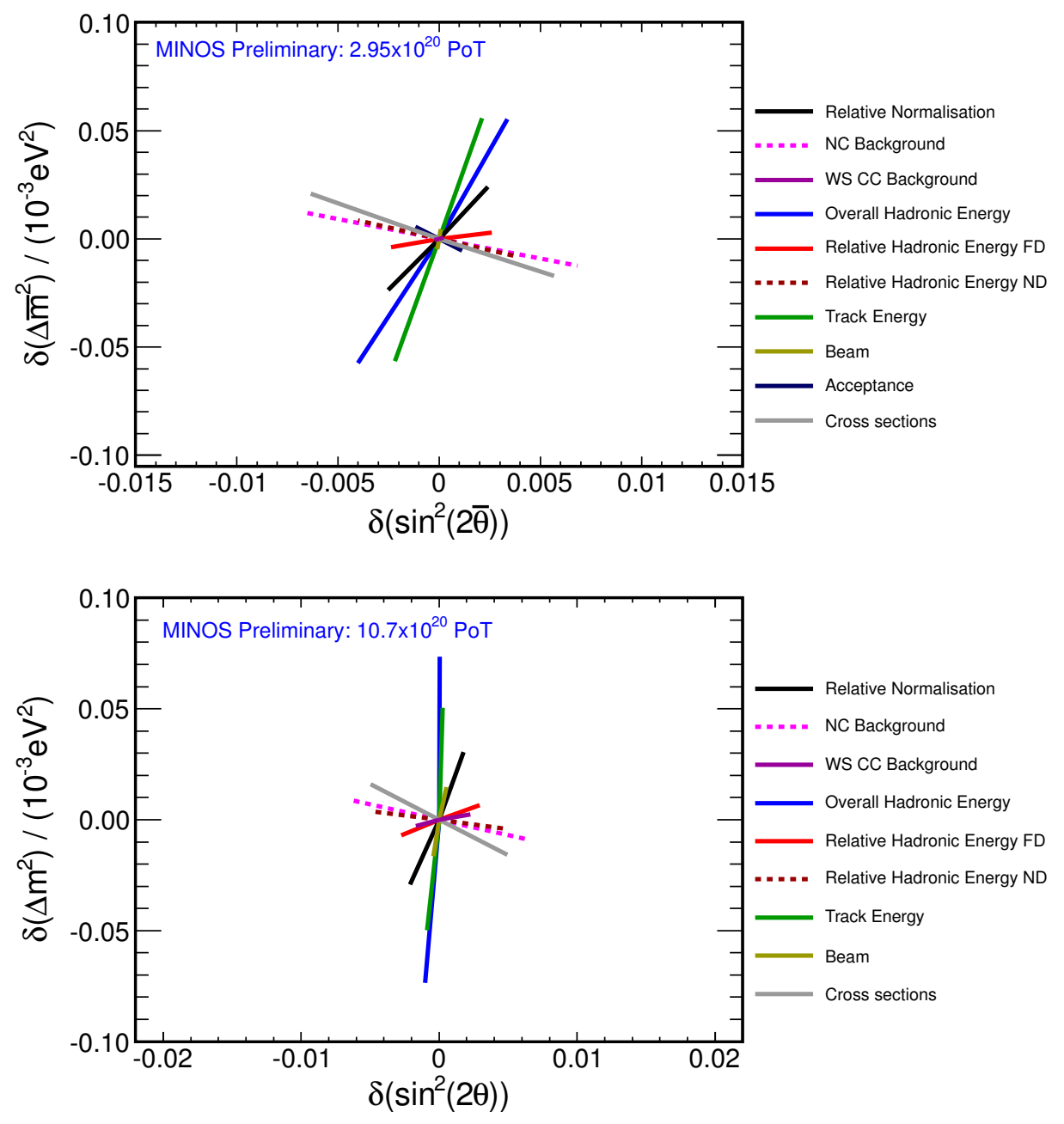

Figure 4.18: The shifts to the best fit oscillation parameters induced by the application of the 2011 NuMuBar RHC analysis (top) and $2012 \mathrm{NuMu} \mathrm{FHC}$ analysis (bottom) systematic shifts to the "fake data". The sum, in quadrature, of all cross section shifts is also shown. 

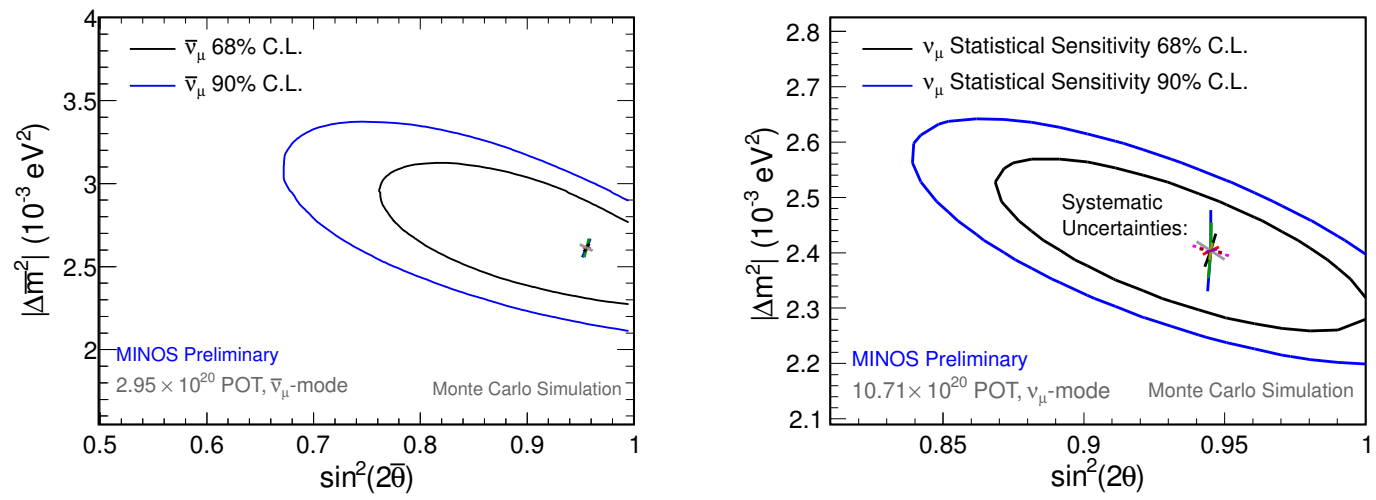

Figure 4.19: The shifts to the best fit oscillation parameters overlaid on the statistical sensitivity contour for the RHC (left) and FHC (right) neutrino analyses.
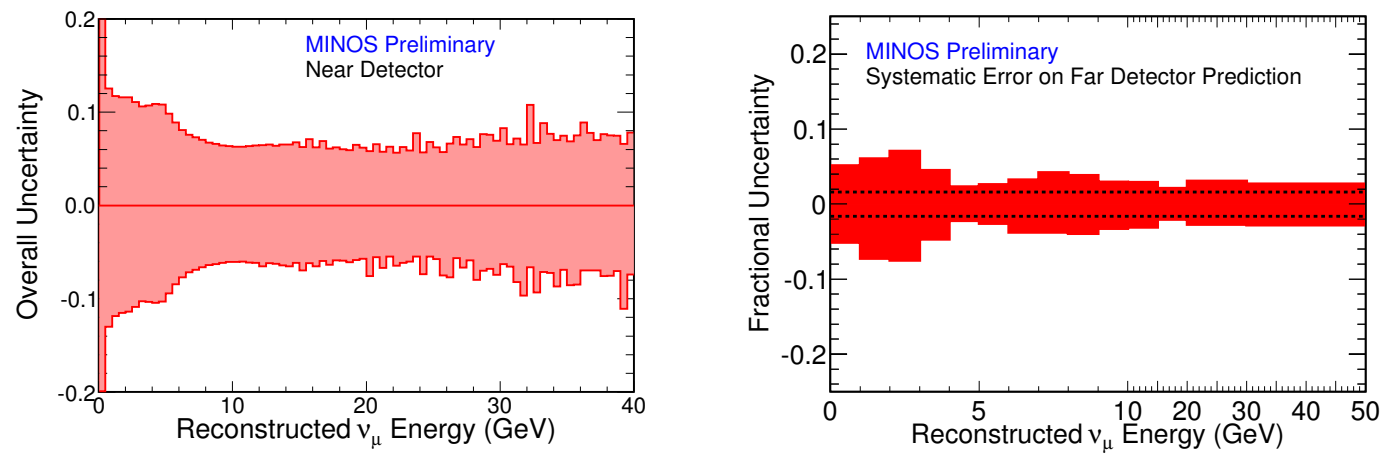

Figure 4.20: The energy dependant systematic uncertainty at the Near Detector (left) and Far Detector (right). 


$$
e_{i}=e_{i}\left(\theta_{23}, \Delta\left|m_{a t m}^{2}\right|\right)
$$

where $i$ is an energy bin of the FD spectrum, $o_{i}$ is the observed event number in that bin, and $e_{i}$ is the expected number of events in that bin for a certain $\Delta\left|m_{\text {atm }}^{2}\right|$ and $\theta_{23}$. When fitting we sum over every bin of every spectra which we analyze.

We fit to a combination of $\Delta\left|m_{a t m}^{2}\right|$ and $\theta_{23}$, so we can construct a "likelihood surface" or grid of log likelihoods in the physically possible region near our best fit point. This allows us to construct confidence levels by using a $\Delta \chi^{2}$ at each point compared to the best fit. Certain values of this "UP value" [49] correspond to degrees of confidence as outlined in Table 4.1, and by drawing contours along lines of confidence we arrive at our confidence intervals. The $2 \mathrm{D}$ surface gives us our best fit point. To estimate the single parameter uncertainty we use the technique of marginalization to convert our 2D likelihood surface into a 1D projection. The $1 \mathrm{D}$ projection of $\theta_{23}$, for example, is found by taking the lowest $\Delta \chi^{2}$ out of the full range of $\Delta\left|m_{\text {atm }}^{2}\right|$ for each value of $\theta_{23}$. The minimum of this 1D projection thus lies at our $\theta_{23}$ best fit point and the confidence levels on the $\theta_{23}$ best fit are then given by reading off the $1 \mathrm{D} \Delta \chi^{2}$ values from Table 4.4 .

\begin{tabular}{lcr}
\hline Confidence Level & $1 \mathrm{D}$ & $2 \mathrm{D}$ \\
\hline $90 \%$ & 2.71 & 4.61 \\
$60 \%$ & 1.00 & 2.30
\end{tabular}

Table 4.1: $\Delta \chi^{2}$ at different confidence levels for a one or two dimensional likelihood surface. 

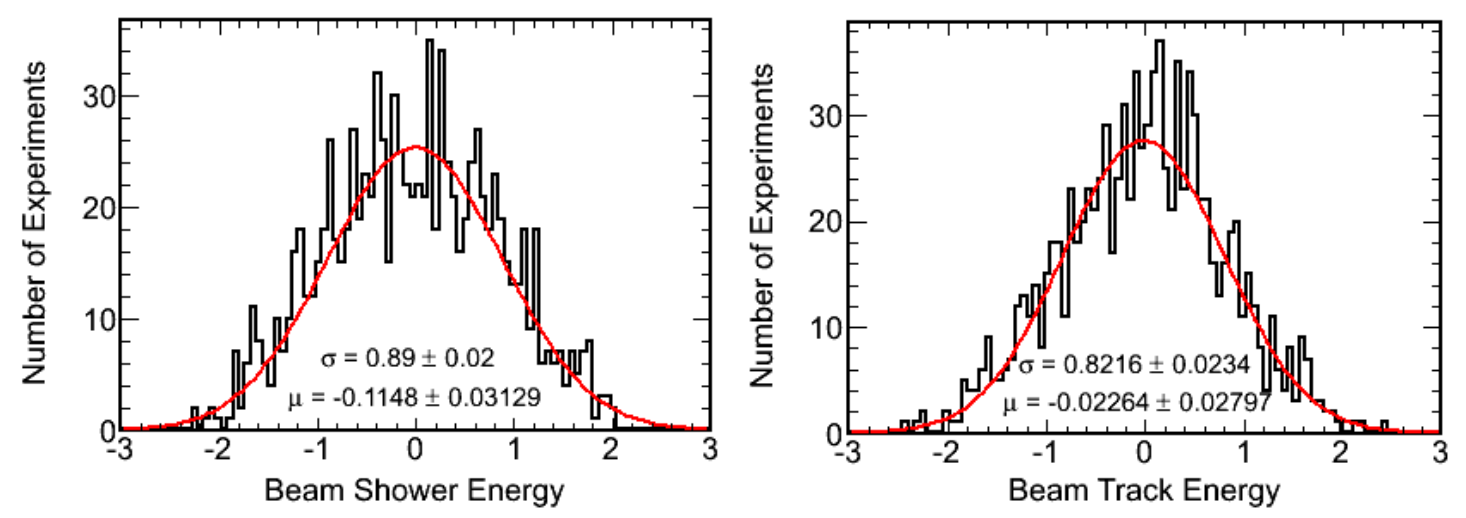

Figure 4.21: The distribution of the major systematics obtained by a two parameter fit on 1000 fake experiments using MINOS Beam Monte Carlo data sets. The distributions of best fit systematics all have a mean value close to zero and width close to unity, and are well-described by Gaussian distributions, indicating no apparent bias or pathology in the two parameter fit. Figure taken from [97.

\subsubsection{Systematic Uncertainty}

\section{Penalty Term}

The above method is adequate for assessing statistical uncertainty on a result, however it does not take into account the effects of systematic uncertainties on the confidence levels. Assuming the systematics follow a Poisson distribution we can quantify our goodness-of-fit to the systematic as:

$$
\chi^{2}=\sum_{j} \frac{s_{j}^{2}}{\sigma_{j}^{2}}
$$

where for $j$ systematics, $s_{j}$ is the best fit to the uncertainty and $\sigma_{j}$ is the $1 \sigma$ systematic uncertainties. The assumption of systematic uncertainties well described by a poisson distribution is well supported by fake experiment experiments which show they fit well to gaussian distributions. Figure 4.21 shows fake experiment distributions for two major disappearance analysis systematics.

Without a bias in the data $s_{j}$ will fit to its nominal value and the further it deviates from Nominal the larger $\chi^{2}$ becomes. If we now include this as a penalty 
term in our original goodness of fit, and make $e_{i}$ also a function of $j$ considered systematic shifts we arrive at:

$$
\begin{gathered}
\chi^{2}=2 \sum_{i} e_{i}-o_{i}+o_{i} \ln \left(\frac{o_{i}}{e_{i}}\right)+\sum_{j} \frac{s_{j}^{2}}{\sigma_{j}^{2}} \\
e_{i}=e_{i}\left(\theta_{23}, \Delta\left|m_{\text {atm }}^{2}\right|\right)
\end{gathered}
$$

where the penalty term is evaluated at every $\theta_{23}$ and $\Delta\left|m_{\text {atm }}^{2}\right|$ combination by marginalizing over the separate systematic parameters. Allowing the systematics to float both improves our goodness of fit whilst also raising the height of the likelihood surface such that our confidence levels, still dominated by statistical uncertainty, become slightly larger. The detailed knowledge of our systematics is thus used to estimate our systematic uncertainty.

\section{Interpolation Over Systematic Uncertainties}

In order to avoid the computational expense of generating a series of full 3D likelihood surfaces for every combination of the oscillation parameters and systematic uncertainty we instead again use the fact that the systematics fit to a simple, approximate Gaussian, distribution to use only 4 grid points to cover the full range for each systematic at every assessed combination of the oscillation parameters [98]. These grid points correspond to $\pm 1 \sigma$ and $\pm 2 \sigma$ systematic shifts and we can interpolate over them to describe a $\chi^{2}$ function that can be given to MINUIT [99] and searced instead of a full extra dimension of the likelihood surface.

MINUIT requires that any such $\chi^{2}$ function be doubly derivable, which is not true if we just linearly interpolate over the 4 shifts. Instead we construct a weighted average over the linear interpolation over the neighbour such that the integer shifts match exactly the value originally created at that grid point and intermediate values are increasingly effected by their neighbours. 


\section{Chapter 5}

\section{The Charged Current Analysis}

The Charged Current or CC analysis at MINOS is the analysis of muon neutrino disappearance in the Forward Horn Current NuMI beam using the MINOS detectors under the assumption of identical neutrino and antineutrino oscillations. It follows the general steps of a disappearance analysis, as outlined in chapter 4 . This chapter will discuss in detail the particular characteristics of this disappearance analysis.

The CC analysis outlined below differs from the 2010 CC analysis [100] in two major ways. First, it represents a gain in the statistical sensitivity by including runs $\mathrm{V}+\mathrm{VI}+\mathrm{X}$ or an extra $3.01 \times 10^{20} \mathrm{PoT}$ of data. Secondly it was the first CC analysis to include the stringent geometric selection criteria at the Near Detector to remove poorly reconstructed events, as discussed in Section 4.1.1. Specifically, we remove tracks which enter or pass near the coil hole, a poorly modelled region of the detector. These events were responsible for a rate of reconstruction failure which disagreed in data and MC. This geometric selection criteria removes a large fraction of the events at the Near Detector (as we are limited by Far Detector statistics this has little effect on the statistical reach of the final analysis), but at the same time eliminates what was previously an unquantified systematic 
uncertainty.

\subsection{Analysis Outline}

The analysis follows all the basic steps outlined in Chaper 4. There are however two major additions, resolution binning and the antifiducial sample. Resolution binning is the process of splitting our analysis samples into separate sub-samples based on the quality of the energy resolution so as to give greater weight to better resolved events and to better constrain the systematic uncertainty. We also include an antifiducial or rock sample of neutrinos which interacted in the rock near the MINOS Far Detector rather than in the detector itself. Both are described in some detail below.

\subsubsection{Resolution Binning}

Neutrino oscillations are an energy dependant phenomena, so it comes as no surprise that the energy resolution of an event is of paramount interest. Better resolution allows us to more sharply define the dip in our FD spectrum from oscillations whilst poorer resolution results in a smearing of this same dip. To that end we quantify the energy resolution at our FD by studying the deviation in true and reconstructed energy. We consider shower energy, track energy from range, and track energy from curvature separately. In each case the distribution of $\left(E_{\text {reco }}-E_{\text {true }}\right.$ against $E_{\text {reco }}$ is used to make a Gaussian fit, and the fitted standard deviation used as an energy resolution estimate [98]. The resolution functions determined by this method are:

$$
\begin{gathered}
\sigma_{\text {trk }}^{\text {range }}=(6.9 \%) E_{\text {range }}+(5.1 \%) \sqrt{E_{\text {range }}} \\
\sigma_{\text {trk }}^{\text {curv }}=p_{\text {curv }}^{2} \sigma_{q / p}+(1.34 \%) \sqrt{p_{\text {curv }}^{2} \sigma_{q / p}} \\
\sigma_{s h w}=(8.6 \%) E_{s h w}+(40.5 \%) \sqrt{E_{\text {shw }}}+275 \mathrm{MeV} .
\end{gathered}
$$


Those separate parametrisations of shower and track energy resolutions as a function of energy are added in quadrature to predict the expected energy resolution of a FD selected event:

$$
\sigma_{t o t}^{2}=\sigma_{t r k}^{2}+\sigma_{s h w}^{2}
$$

We then use this resolution parametrisation to divide the $\nu_{\mu}$ sample we have selected at the FD into bins of energy resolution. These resolution quantiles are defined to divide an event at any given reconstructed energy into 5 equal components, shown in Figure 5.1, with equal statistics for the unoscillated scenario. Separate selection criteria are chosen for each individual run period as each beam configuration has a distinct energy spectrum. Energy resolution for our highest and lowest resolution samples can be seen in Figure 5.2 .

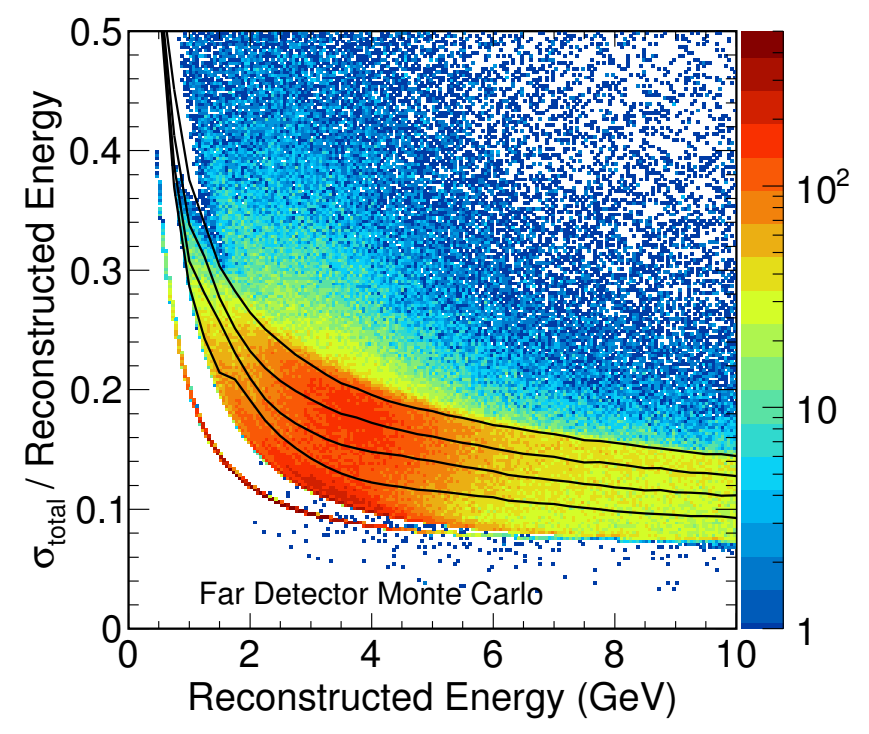

Figure 5.1: $\sigma_{\text {total }} /$ Reconstructed Energy, the energy resolution as a fraction of total reconstructed energy, vs. the reconstructed energy of selected events for the null oscillation case at the Far Detector. The boundaries between the five energy resolution quantiles are shown in black and each bin of resolution contains the same number of events. Quasi-elastic events with zero shower energy appear as a band of events at the low edge of the distribution. 

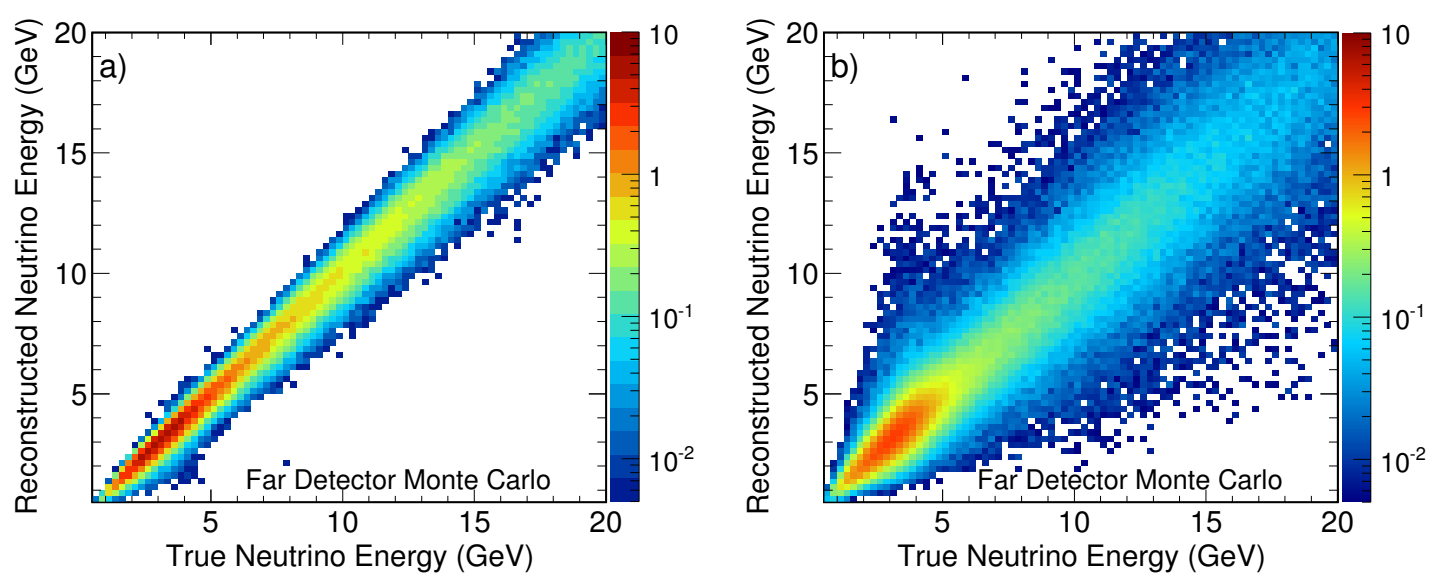

Figure 5.2: The Reconstructed vs. True energy matrices for the highest (left) and lowest (right) energy resolution quantiles.

\subsubsection{The Rock and Antifiducial Sample}

The majority of MINOS analyses make use of a preselection selection criteria on the fiducial volume, only accepting events which begin at a certain depth within the detector. Events failing this selection criteria are rejected because of the poor energy resolution and broadly fall into the category of "Rock" or "Antifiducial" events. Antifiducial events originate near the edge of the detector and cannot guarantee complete shower containment which severely limits the shower energy resolution. Rock events start in the rock surrounding the detector, and are usually completely missing their shower component and some unknown portion of their track component such that the track and shower energy resolution is extremely poor. Figure 5.3 shows the distribution of true neutrino vertices in the FD cavern.

However, whilst the energy resolution of these events is extremely poor they are a high statistics sample at the FD, and hence this rock and antifiducial (RAF) sample represents a valuable addition to the oscillation measurement. Due to the lack of visible energy an energy estimator, based on the observed muon track energy, is used. Whilst the lack of a shower energy component severely limits 
the final energy resolution, the muon track energy is correlated to the true neutrino energy so we retain some information. In the event that some shower does reach the detector the roID and jmID NC/CC separation parameters are used as usual. Without a near RAF sample cross section systematics become far more important, however they remain dwarfed by the now far larger track and shower energy systematics.

Energy resolution in the RAF sample varies greatly depending on where the event originates and hence how much unknown energy might have been have lost, with the antifiducial samples the highest resolution because the muon track is fully contained. In analogy to the resolution binning in the fiducial sample the RAF sample is further split into 6 subsamples shown in Figure 5.4 based on the detector location where the first hit is recorded. Each area samples a particular type of event based on geometrical considerations. Events at the front face of the detector are predominantly rock events, whilst those in the gap and back regions are predominantly antifiducial events. Edge events are seperated into rock or antifiducial events based on the precise sequence of hits at the track start and arrangement of strips at the detector edge.

The extrapolation and prediction use the standard ND fiducial sample to create a prediction of the Far Detector flux. This Far Detector flux is then uses as a scale on the RAF MC rather than being directly converted into a FD prediction. This method is a slight alteration on the standard extrapolation procedure and allows the full power of our ND sample to be leveraged. The RAF sample at the $\mathrm{ND}$ is of little interest since the neutrino rates are high enough that the extra statistics are unnecessary. 


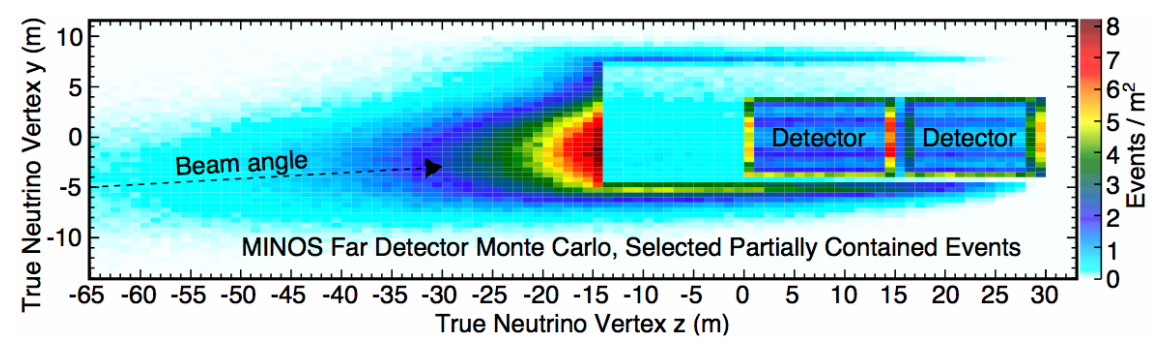

Figure 5.3: True interaction vertices of events selected into the RAF sample, taken from MC. Both the structure of the cavern and the supermodules is visible, with the majority of events coming from rock interactions upstream of the detector.

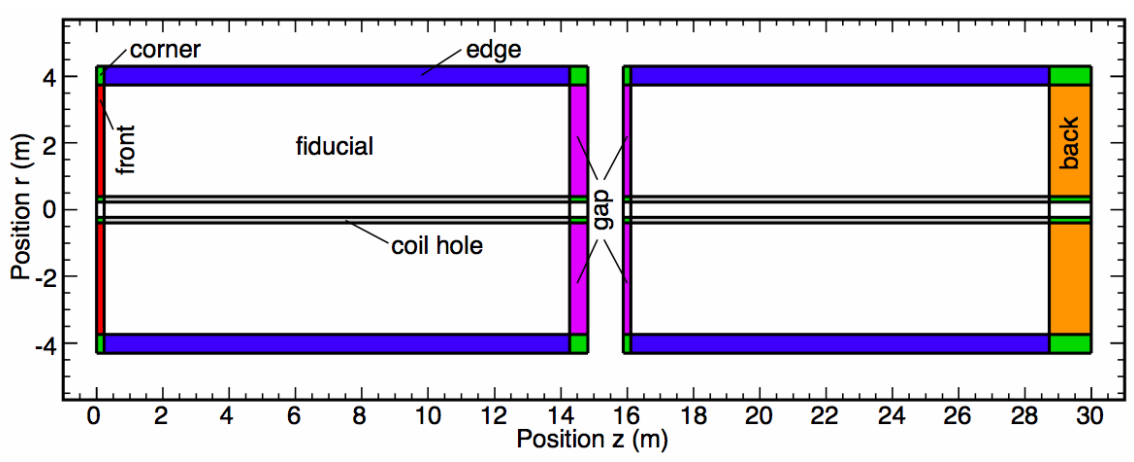

Figure 5.4: Scale diagram showing the detector regions (coloured) used to separate events in the RAF sample

\subsubsection{Addition of the ND Coil Hole Selection Criteria}

The 2012 Charge Current analysis differs from the 2010 analysis mainly in the application of a new Near Detector selection criteria, described in section 4.1.1. The effect of this change on the FD spectrum can be seen in Figure 5.5 which compares the combined FD predicted spectrum (from runs I, II , III, and IpHE ND data) as calculated with the 2010 and 2012 analyses. As expected the difference is subtle. 

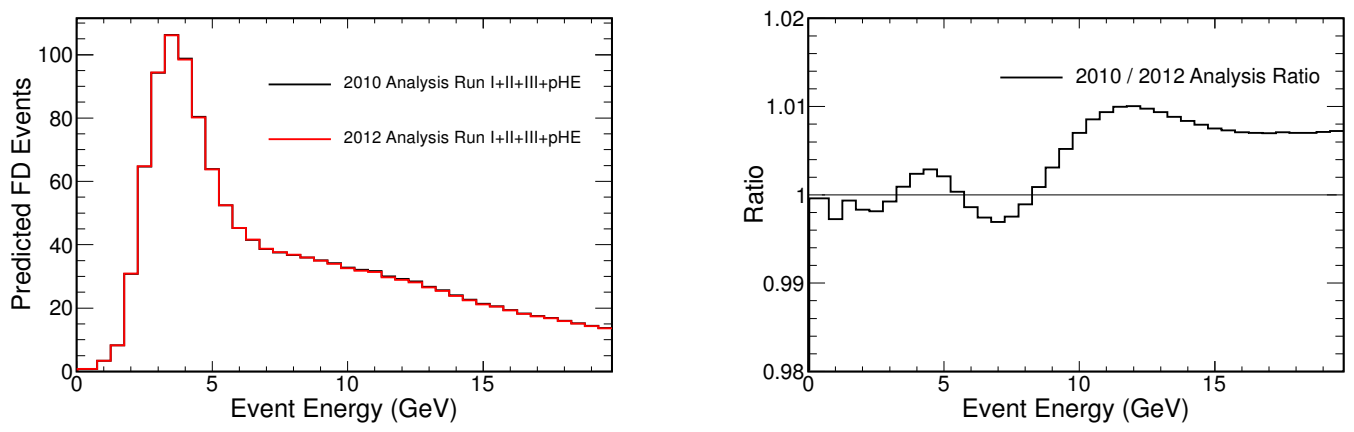

Figure 5.5: Predicted spectrum of selected $\nu_{\mu}$ events in the Far Detector (left), ratio of spectrum as measured by different analyses (right).

Our systematic uncertainties, because of the new ND selection criteria, have grown slightly which is evident in Figure 5.6. Particularly track and shower energy by $27 \%$ and $36 \%$ respectively. This quantifiable growth is, however, desirable as it replaces an unquantified uncertainty from reconstruction mismodelling.

\subsection{Data Epochs}

The MINOS data sets and detector live times during beam operation are descibed in Ref.[101]. The methodology for counting the accumulated Protons on Target (PoT) used in this analysis is described in Ref. 102. The run periods used in this analysis and their associated PoTs are summarised in Table 5.1 . 

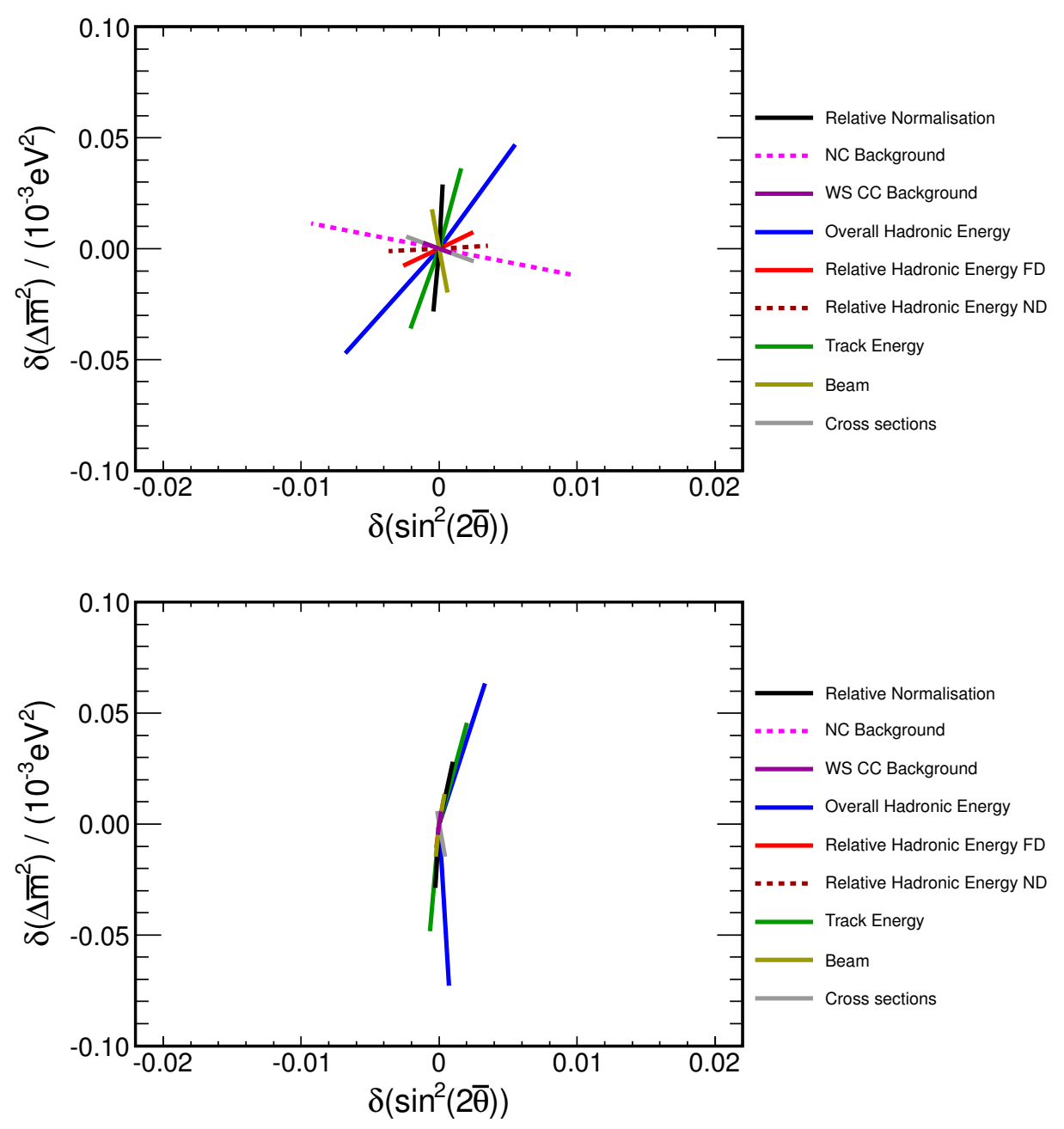

Figure 5.6: The major sources of uncertainty in the 2010 (top) and 2012 analysis (bottom). 


\begin{tabular}{l|l|l} 
Run Period & Beam Type & PoT $\left(\times 10^{18}\right)$ \\
\hline Run I & LE & 126.93 \\
\hline Run I & pHE & 15.31 \\
\hline Run II & LE & 194.27 \\
\hline Run III & LE & 388.09 \\
\hline Run V & LE & 45.788 \\
\hline Run VI & LE & 56.600 \\
\hline Run X & LE & 147.17
\end{tabular}

Table 5.1: Run Periods showing accumulated PoT in the Far Detector. Beam types as described in Section 3.2.

\subsubsection{Reweighting}

There are two distinct changes in the neutrino flux between the different LE FHC Run periods. The first being the shift to the spectrum from Run III onwards when helium was added to the decay pipe. The other being the effect of target decay over time, which effected Run III. This is accounted for in the MC by the addition of helium into our MC simulations from Run III onwards, and an energy dependant decay reweighting [85] of the Run III MC to simulate the effect of target decay. Table 5.2 contains information about any notable changes between the runs. 


\begin{tabular}{l|l|l} 
Run Period & Beam Type & $\begin{array}{l}\text { Beam Condi- } \\
\text { tions }\end{array}$ \\
\hline Run I & LE & Nominal \\
\hline Run I & pHE & Nominal \\
\hline Run II & LE & Nominal \\
\hline Run III & LE & Helium + Target \\
& & Decay \\
\hline Run V & LE & Helium \\
\hline Run VI & LE & Helium + Bent \\
& & Target \\
\hline Run X & LE & Helium
\end{tabular}

Table 5.2: Run Periods with extra information about conditions during the runs.

In the $2010 \mathrm{CC}$ analysis we only required beam reweighting for either no helium and target decay (Runs I and II) or both (Run III). However Runs V/VI/X required a weighting with helium but without target decay. Figure 5.7 shows the success of the reweighted MC with and without target decay included in replicating the difference between two data runs only differentiated by target decay. 


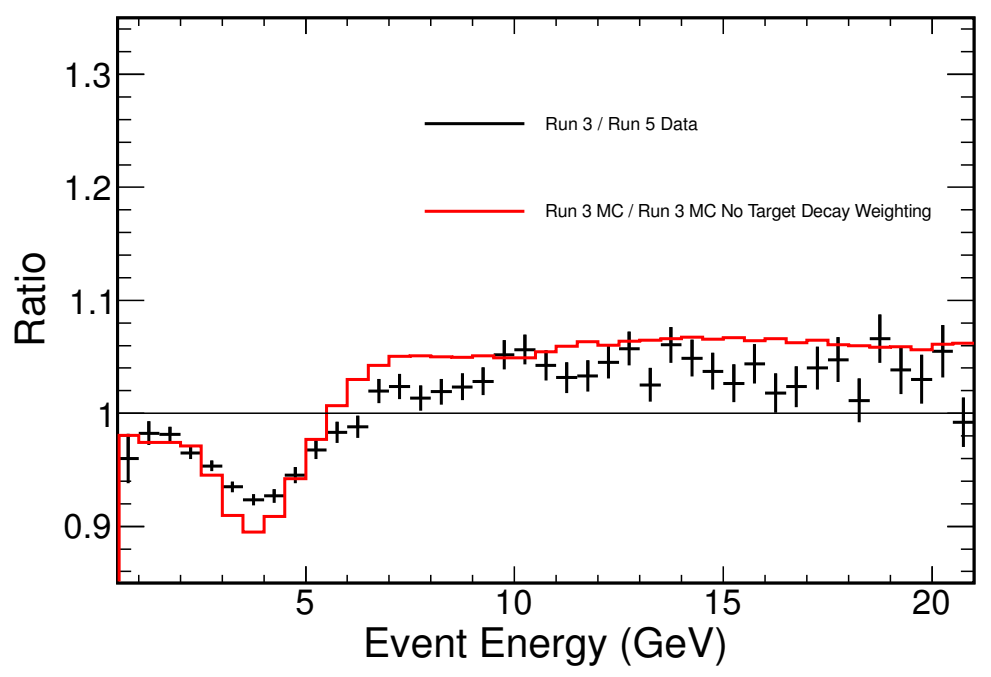

Figure 5.7: Ratio of ND muon neutrino spectrum in Runs 3 and 5 alongside the ratio of the Run $3 \mathrm{MC}$ with and without target decay weighting.

\subsection{Analysis Fit}

The CC analysis follows the general fitting outline described in Section 4.4. Each FHC run of the NuMI beam is considered seperately, with each run further broken down into 5 resolution bins, the sample of positive charge sign events (antineutrinos) and the RAF sample. Each sample passes through the event selection and extrapolation procedures outlined in this and the previous chapter and the assumption of identical neutrino and antineutrino oscillations is made. The four largest systematic uncertainties, as assesed in Section 4.3.1, of shower energy, track energy, neutral current background and normalization are all included using the method described in Section 4.4.1. , 


\subsubsection{Result}

The full Near Detector data set shows good agreement between data and tuned MC, as seen in Figure 5.8. There are fewer PoTs in the Near than Far Detector Data set because the Near Detector set was closed off several months earlier than the Far Detector to allow time for calibration of the both Near and Far detector samples before the Neutrino 2012 conference. This strategy is possible because the Near Detector dataset is not statistically limited, and the beam remained stable for the full duration each run used. Extrapolating to a Far Detector prediction and fitting for the full FHC dataset as described we find a best fit to the atmospheric mixing parameters of $\Delta\left|m_{\text {atm }}^{2}\right|=2.42 \times 10^{-3} \mathrm{eV}^{2}$ and $\sin ^{2}\left(2 \theta_{23}\right)=0.936$. Figure 5.9 and 5.10 show the observed spectrum and predicted spectrum made at our best fit oscillation parameters and Figures 5.11 and 5.12 shows the constraints that this fit places on the possible values of the atmospheric mixing parameters. 3564 events are expected without oscillations, and 2894 were observed. Assuming oscillations at the neutrino best point, we would expect 2814.7 events. Table 5.3 shows the complete event count breakdown. 


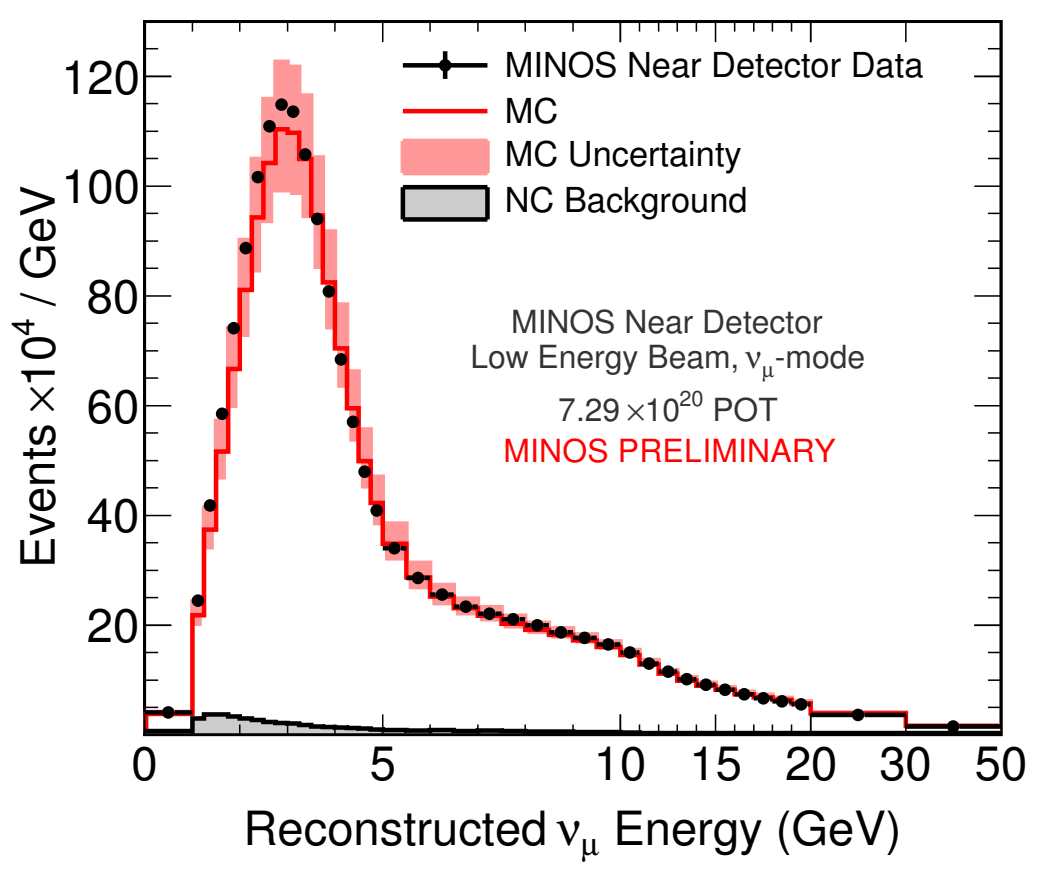

Figure 5.8: Reconstructed energy distribution of events selected as neutrinos in the Near Detector. The red histogram represents the Monte Carlo expectation with the systematic error, the blue histogram represents the total neutral current background, and black points represent data. 


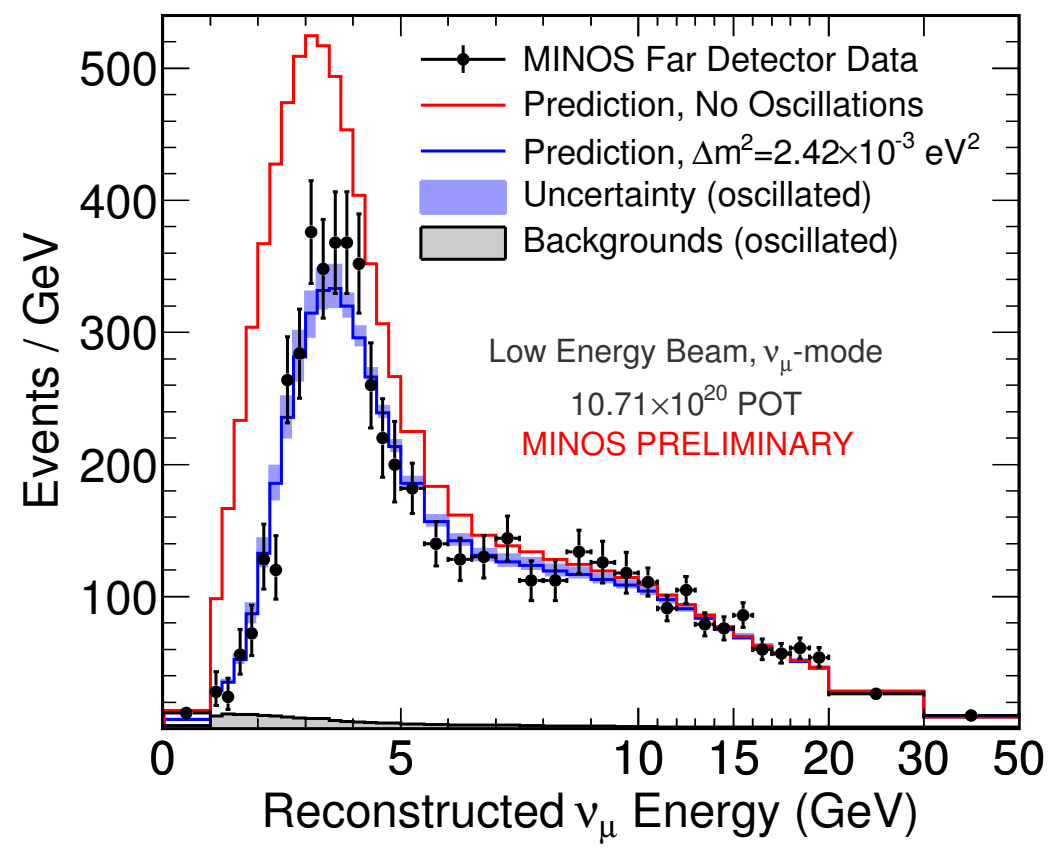

Figure 5.9: Far Detector data and predictions with no oscillations (red histogram) and with the best-fit oscillation parameters (blue histogram). The band around the oscillated prediction represents the total systematic uncertainty. Total background in the oscillated prediction is also displayed (gray shaded histogram). The data and predictions correspond to an exposure of $10.7 \times 10^{20}$ PoT. 


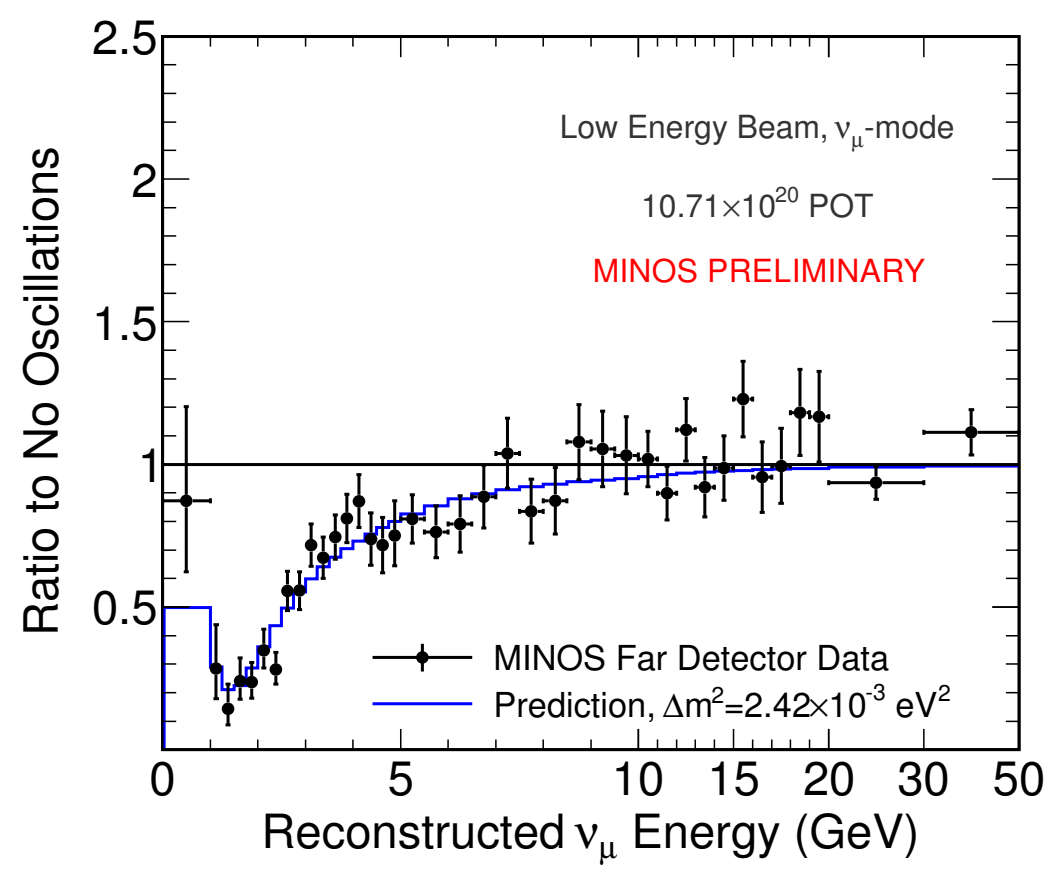

Figure 5.10: Ratio of Far Detector data and best-fit prediction to no oscillations. The data and predictions correspond to an exposure of $10.7 \times 10^{20}$ PoT. 

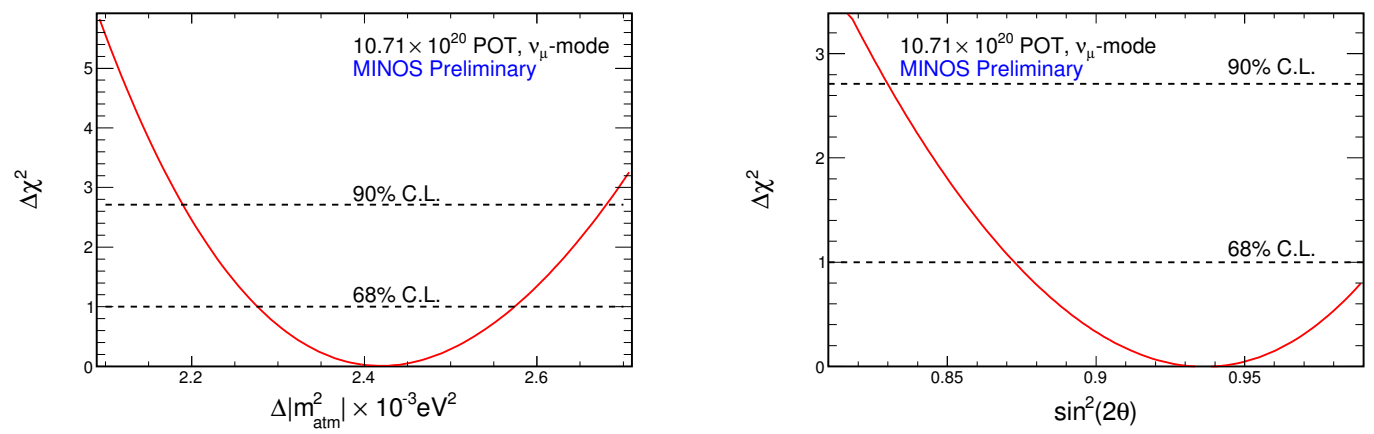

Figure 5.12: $1 \mathrm{D}$ marginalizations of our full contour for $\Delta \chi^{2}$ for $\Delta \mid m_{a m}^{2}$ (left) and $\sin ^{2} 2 \theta_{23}$ (right). Dashed lines show $68 \%$ and $90 \%$ C.L.

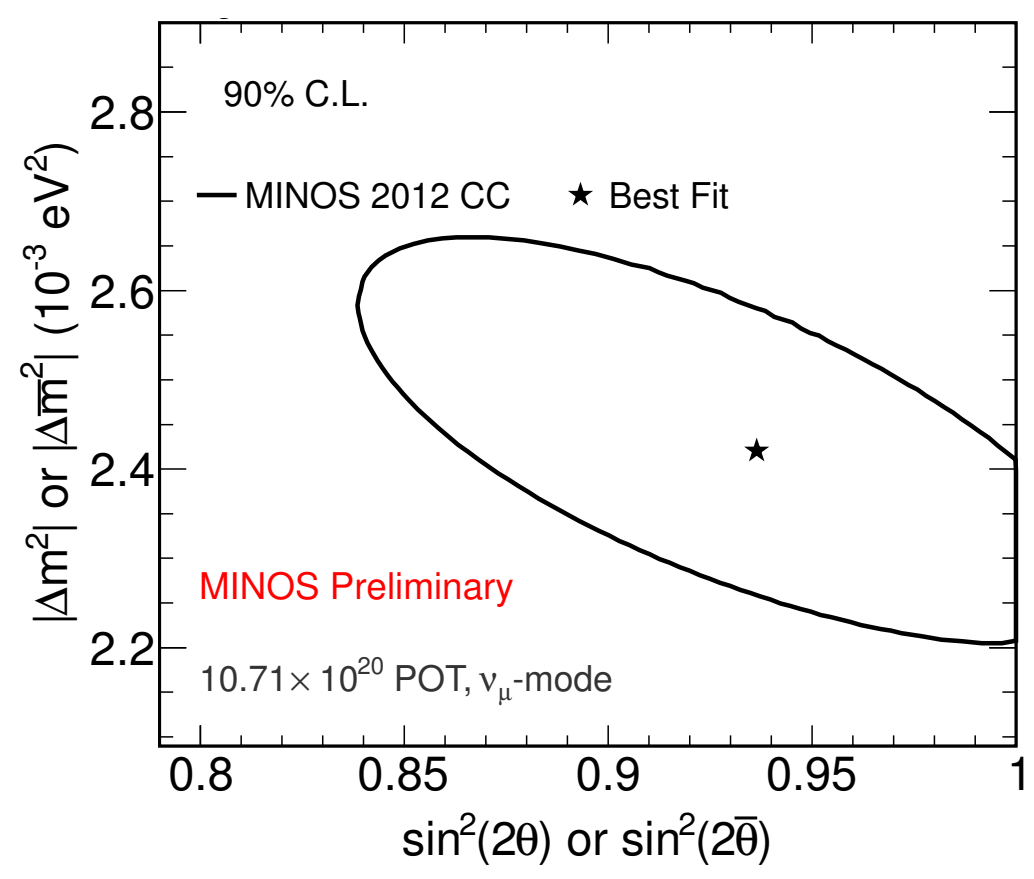

Figure 5.11: The 90\% 2012 CC Analysis contour from FHC running. The best fit is at $\Delta\left|m_{\text {atm }}^{2}\right|=2.42 \times 10^{-3} \mathrm{eV}^{2}, \sin ^{2}\left(2 \theta_{23}\right)=0.936$ 


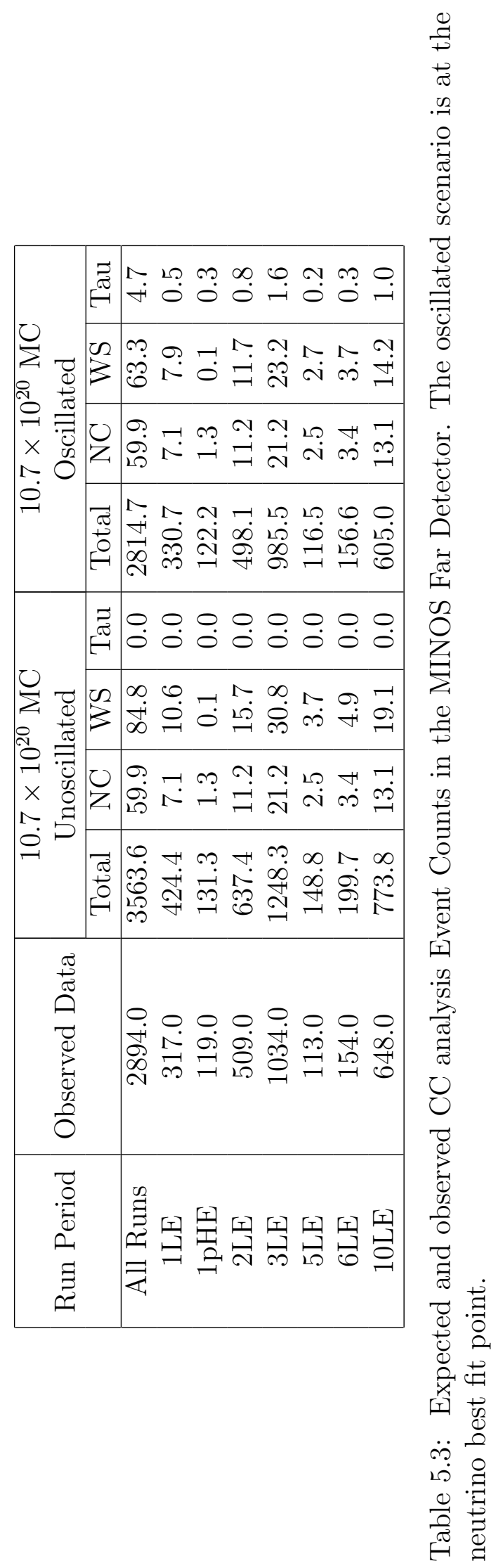




\section{Chapter 6}

\section{Three Flavour Oscillations}

This chapter outlines the changes made to the MINOS CC analysis to fit the exact three flavour description of neutrino oscillations rather than a simple two flavour approximation. Additionaly, a fit to the exact atmospheric mixing parameters will be shown.

\subsection{Analysis Overview}

The 2013 CC analysis is largely the same as the 2012 CC analysis (detailed in chapter 5) with the exception that it now uses an exact solution of the three flavour neutrino oscillation model to make the final fits to the data rather than the previous two flavour oscillation model. The motivation for this update does not come from any signficant alterations to our best fit with these new parameters, in fact this chapter will show we are largely insensitive to the other mixing

parameters. Rather with the measurement of a non-zero $\theta_{13}$ by Daya Bay [52] last year the field is moving towards using the more rigorous exact solutions. By updating our result we will facilitate direct comparison and combination of the world's knowledge of $\theta_{23}$ and $\Delta m_{32}^{2}$. 
As part of this change the analysis also moves to a finer binning in true energy at low energies. This is necessary as it is at those low energies that differences between these two models begin to manifest themselves in our analysis, and these same oscillations are rapid enough at those low energies that we require finer binning to fully study the region.

\subsection{The Exact Three Flavour Oscillation For- mula}

The previous analysis used an approximation of the muon neutrino survival probability derived from the full three flavour solution (without matter effects):

$$
P\left(\nu_{\mu} \rightarrow \nu_{\mu}\right) \approx 1-\sin ^{2}(2 \theta) \sin ^{2}\left(\frac{1.27 \Delta m_{a t m}^{2} L}{E}\right)
$$

This approximation is based on a series of well grounded assumptions and a full proof is available in Section 2.5.2. However with the measurement of a non-zero $\theta_{13}$ the more rigourous exact solution becomes absolutely necessary for studies of atmospheric neutrinos such as Ref.[103]. Far longer baselines through matter make an atmospheric analysis more sensitive to deficiencies in the two flavour approximations, due to the effect of matter effects. To facilitate the direct comparison and combination of our result internally (with our own atmospherics analysis) and externally (Super Kamiomade, et al) we instead now use an exact solution based on multipication of the PMNS matrix with matter effects.

Figure 6.1 shows the discrepancy between the approximate and exact solutions as a function of length and energy. Clearly they are in very good agreement for the MINOS beam L/E (peak at $3 \mathrm{GeV}$ and $735 \mathrm{~km}$ baseline) but represent a more significant change for the length and energy scales important for other neutrino experiments, such as the analysis of atmospheric neutrinos. 

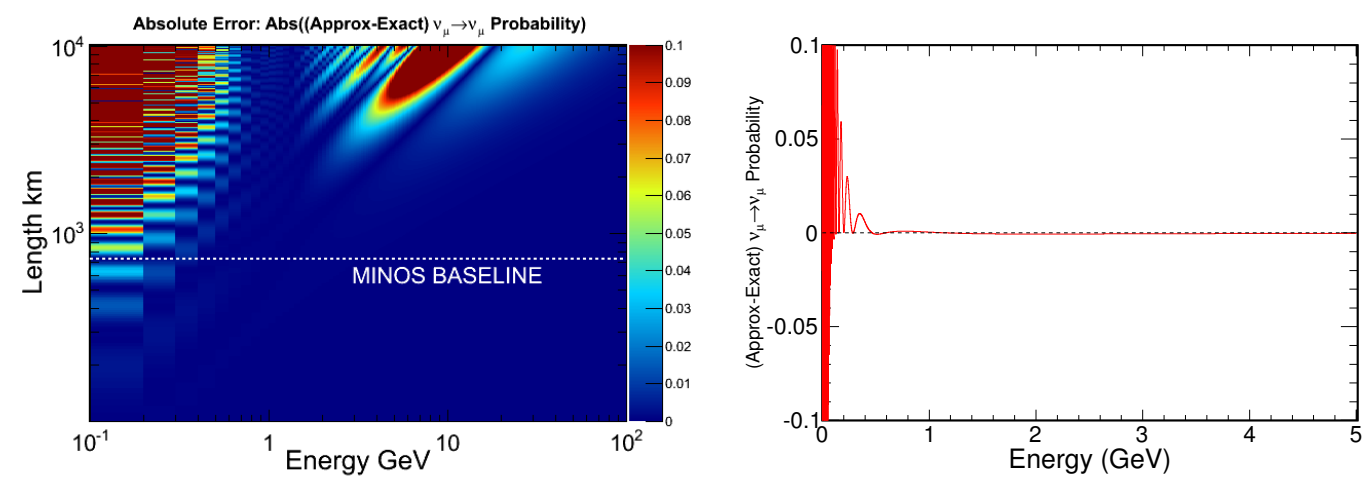

Figure 6.1: On the left is the absolute difference between two flavour approximate and three flavour exact oscillation probabilities as a function of length and energy. On the right the difference as a function of energy for the MINOS baseline of 735 $\mathrm{km}$. Nonzero $\theta_{13}$ and maximal $\theta_{23}$ are assumed.

\subsection{New Truth Binning}

An important stage in the analysis of our data is the reweighting of the prediction of the Far Detector true energy spectrum by different oscillation probabilities using either the bin centre of each energy bin or an interpolation over the entire spectrum. This has been sufficient in the past to give us less than $1 \%$ disagreement between Far Detector "fake data" and predictions in "fake data" studies at most energies and most importantly surrounding the peak of our beam $(3 \mathrm{GeV})$. However, in the sub $1 \mathrm{GeV}$ region poor agreement is seen, this is because at these lower energies the oscillation probility varies rapidly and hence even a sophisticated interpolation technique has a hard time describing the average oscillation probability over our comparitively huge $0.25 \mathrm{GeV}$ bins.

For this analysis we strive for less than $1 \%$ disagreement across the board as it is at very low energies we would expect to see any difference between the two 

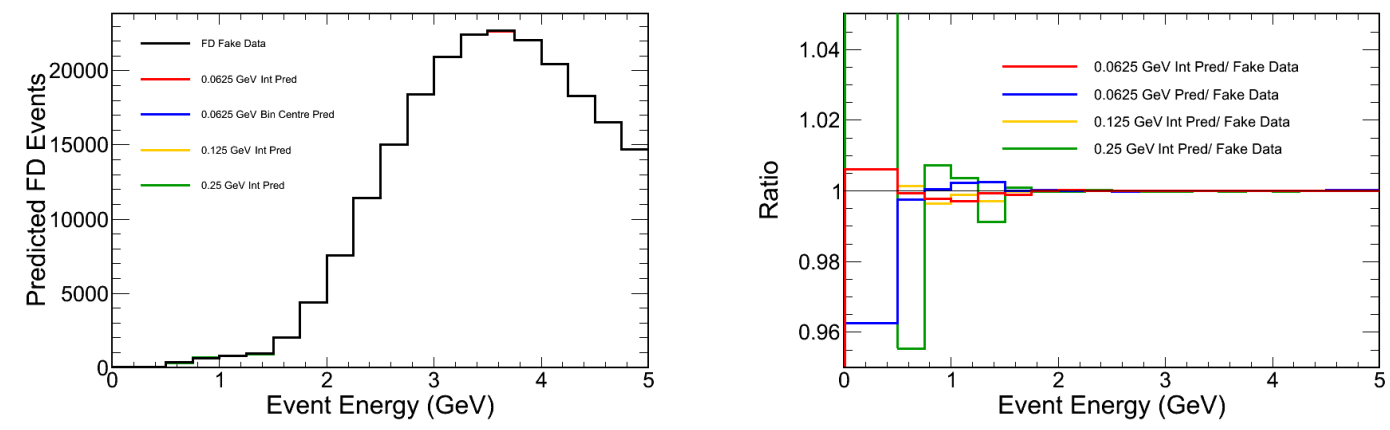

Figure 6.2: Predicted and fake data Far Detector Spectra (left) and the ratio of fake data over prediction (right) for oscillated Far Detector MC. Not that the finer binning and no interpolation (blue) is equivalent to the combination of fine binning and higher order interpolation (red).

and three flavour oscillation formulae manifests itself. Two possible techniques for achieving this were explored, the first was the use of a higher order interpolation to try and better model the rapid low energy oscillations. The second was the use of finer truth binning. Figure 6.2 shows the result of these studies.

Ultimately, it was decided that the best solution was to move to far finer truth binning in the sub $5 \mathrm{GeV}$ region (changing from 0.25 to $0.05 \mathrm{GeV}$ ). This choice is based on the fact that far finer truth binning provides a similiar improvement to the combination of interpolation and finer binning, with the advantage of far less computational expense. The binning in reconstructed energy remains unchanged, as the low energy region is extremely statistics poor. Figure 6.3 below shows the improvement in the agreement between our "fake data" and predicted Far Detector reconstructed neutrino energy spectra when we move from the old coarse truth binning to the finer truth binning. 

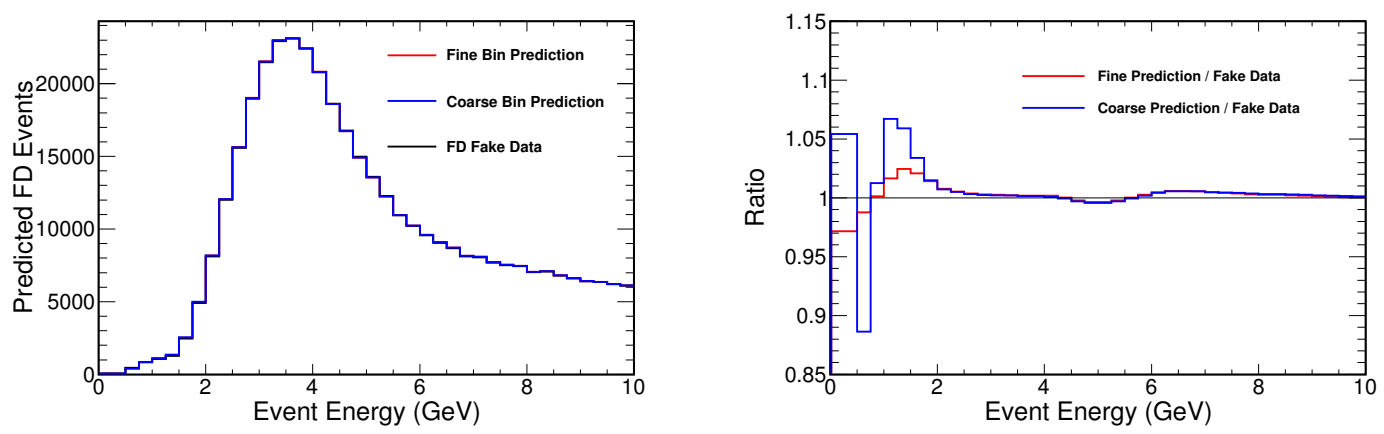

Figure 6.3: Oscillated FD Fake Data and predicted reconstructed energy spectra for the old coarse and the new fine truth binnings left, ratios of predictions against fake data on the right.

\subsection{Fitting Choices}

The move to a three flavour framework necessitates some choices about how to best display our result. These choices come down to how to handle the octant dependancy of $\theta_{23}$ and how to use the worlds knowledge of the mixing parameters that MINOS is less sensitivie to.

\subsubsection{Octant Dependency}

Previously, in our two flavour approximation, the magnitude of muon neutrino disappearance was related to $\sin ^{2}\left(2 \theta_{\text {eff }}\right)$. This meant that any measure of non maximal $\theta_{\text {eff }}$ has a degeneracy between $\theta_{\text {eff }}>\pi / 4$ and $\theta_{\text {eff }}<\pi / 4$ which is impossible to resolve. However a simple first order expansion of the muon dissappearance probability without matter effects is:

$$
\sin ^{2}\left(2 \theta_{\text {eff }}\right)=\sin ^{2}\left(2 \theta_{23}\right)-4 \sin ^{2}\left(\theta_{13}\right) \times\left(\sin ^{2}\left(\theta_{23}\right)-2 \sin ^{4}\left(\theta_{23}\right)\right)
$$

This is important because it means that any fit for $\sin ^{2}\left(2 \theta_{23}\right)$ with a non zero $\theta_{13}$ is sensitive to the selected octant, when you move away from maximal mixing. Figure 6.4 and 6.5 show both how the symmetry of $\sin ^{2}\left(\theta_{23}\right)$ moves slightly away from 0.5 and how this leads to different sensitivity limits for $\sin ^{2}\left(2 \theta_{23}\right)$ depending 

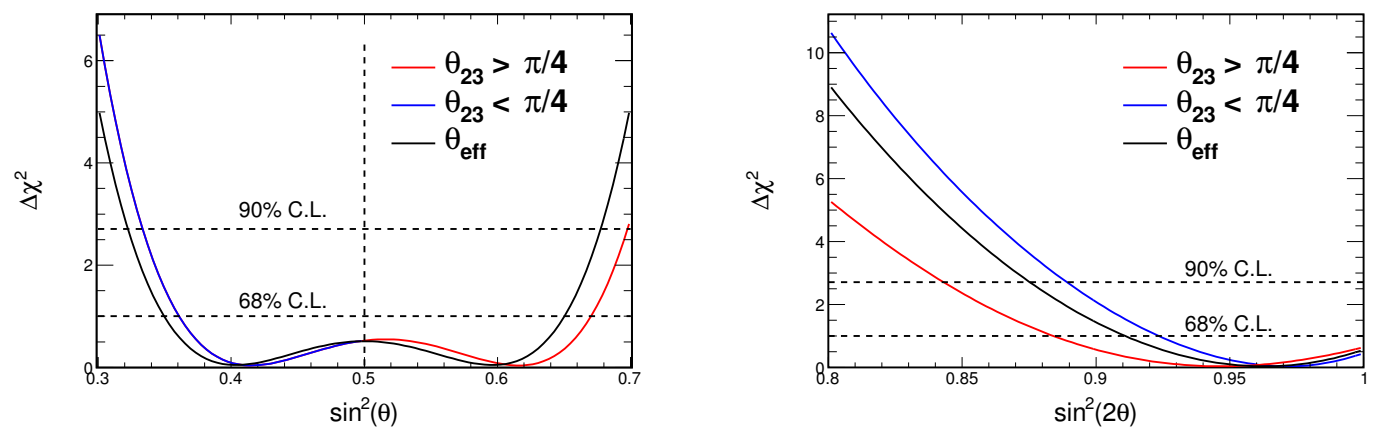

Figure 6.4: The plot on the left shows $-2 \Delta \log (L)$ as a function of $\sin ^{2}\left(\theta_{23}\right)$ and $\sin ^{2}\left(\theta_{\text {eff }}\right)$. On the right $-2 \Delta \log (L)$ as a function of $\sin ^{2}\left(2 \theta_{23}\right)$ in each octant and $\sin ^{2}\left(\theta_{\text {eff }}\right)$.
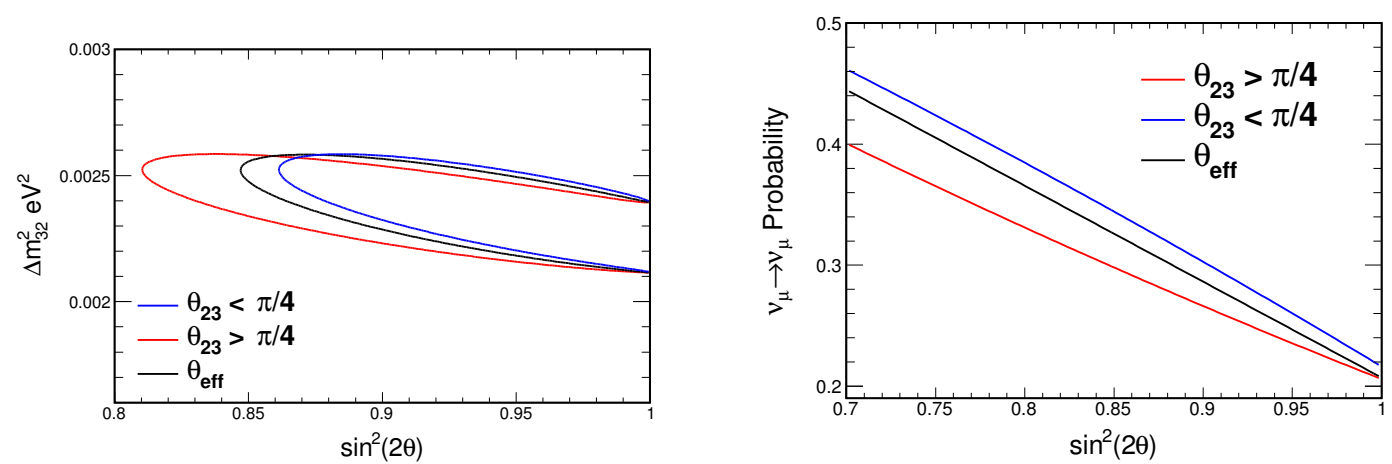

Figure 6.5: The plot on the left shows 90\%C.L. contours for $\Delta m_{32}^{2}$ and $\sin ^{2}(2 \theta)$. The plot on the right shows muon neutrino survival probability as a function of $\sin ^{2}\left(2 \theta_{23}\right)$ and $\sin ^{2}\left(2 \theta_{\text {eff }}\right)$. The different size contours in the left most plot is a direct consequence of the different octant oscillation probabilities in the rightmost plot.

on your choices of octant.

Ultimately this provides a compelling argument for making the fit to $\theta_{23}$ or $\sin ^{2}\left(\theta_{23}\right)$ instead of $\sin ^{2}\left(2 \theta_{23}\right)$. Rather than making an arbitary choice of octant for your final result, instead we show the full phase space. 


\subsubsection{The Effective and Exact Mass Splitting}

In the two flavour approximation without matter effects we fit to the atmospheric mass splitting $\Delta m_{\text {atm }}^{2}$ which is approximately[104]:

$$
\Delta m_{\text {atm }}^{2}=\frac{1}{2}\left|\Delta m_{32}^{2}+\Delta m_{31}^{2}\right|
$$

and hence insensitive to the mass hiearchy. Using the exact three flavour solution we must choose to fit to either $\Delta m_{32}^{2}$ or $\Delta m_{31}^{2}$ and perform the fit for each of the normal and inverted mass hiearchies.

\subsubsection{Matter Effects and The Density of the Crust}

As we now use the exact three flavour neutrino oscillation model with matter effects, we must make a choice about the density of matter bewteen FNAL and the Soudan Mine in Minnesota. We chose an electron density of $N_{e^{-}}=1.36$ moles $/ \mathrm{cm}^{3}$ which is a approximation based on the CRUST 2.0 model [105]. It has already been shown by the MINOS UP group [106] that even for their long baselines they are largely insensitive to uncertainties in the matter density. The full derivation of the electron density used is described in appendix A.

\subsubsection{Fixing or Floating Mixing Parameters}

$\theta_{13}, \theta_{12}$, and $\Delta m_{21}^{2}$ are all well constrained by the global community and we have chosen to make use of this knowledge in our final fit. As is shown in Figures 6.6 and 6.7 we are not particularly sensitive to alterations in these variables within their PDG[49] limits, generally they cause a change in our best fit somewhat smaller than the systematic errors that we take into account in the fit. 


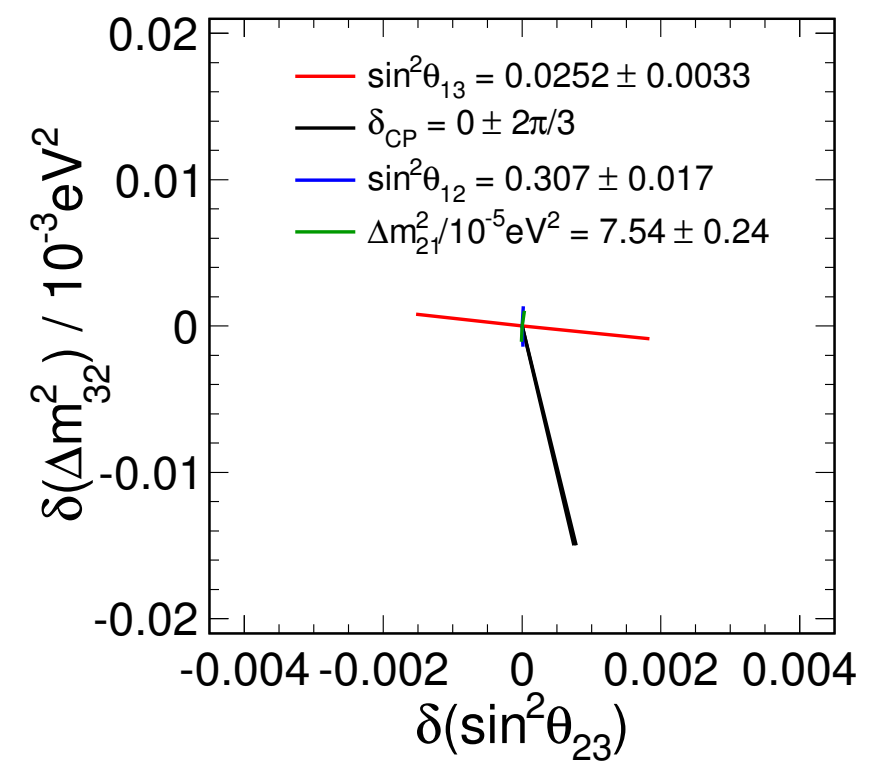

Figure 6.6: A starplot showing the effect on our best fit as we vary $\theta_{13}, \theta_{12}$, and $\Delta\left|m_{21}\right|^{2}$ over their PDG uncertainties and as we vary $\delta_{c p}$ over 0 to $2 \pi$

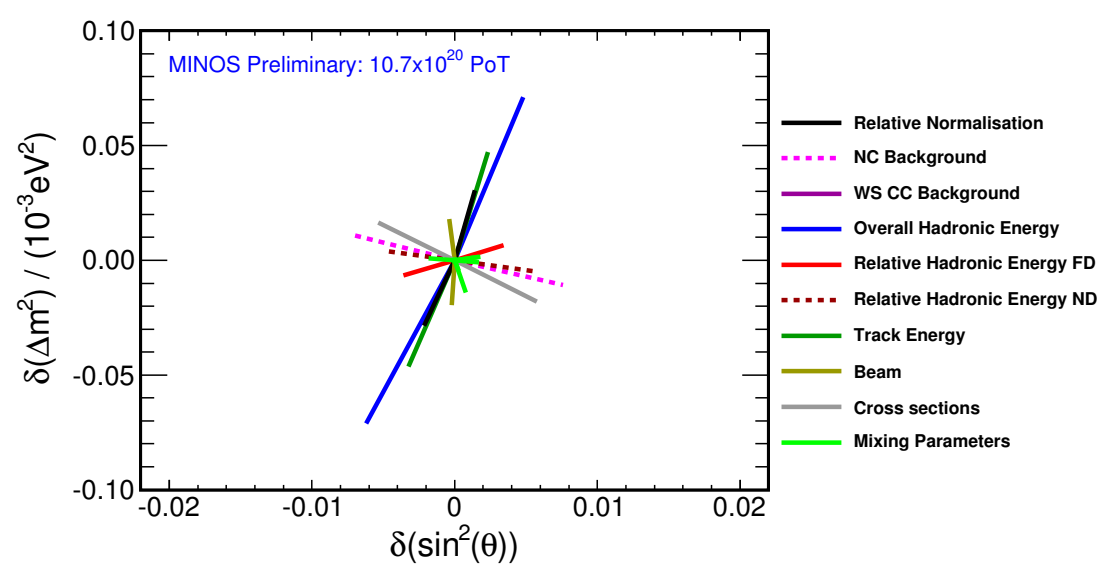

Figure 6.7: A starplot showing in contrast the effect of systematics, the solar and reactor parameters have been set to bright green to make their relative size clear.

However, the new mixing parameters, $\delta_{c p}$ and $\theta_{13}$ have comparatively large effects on the CC analyses best fit. Furthermore it can be shown that if you allow the fit to $\theta_{13}$ and $\delta_{c p}$ to float, whilst the value of $\theta_{13}$ stays flat as a function of $\theta_{23}$ 
and $\Delta m_{\text {atm }}^{2}$ the value of $\delta_{c p}$ changes considerably as shown in Figure 6.8 and 6.9 .

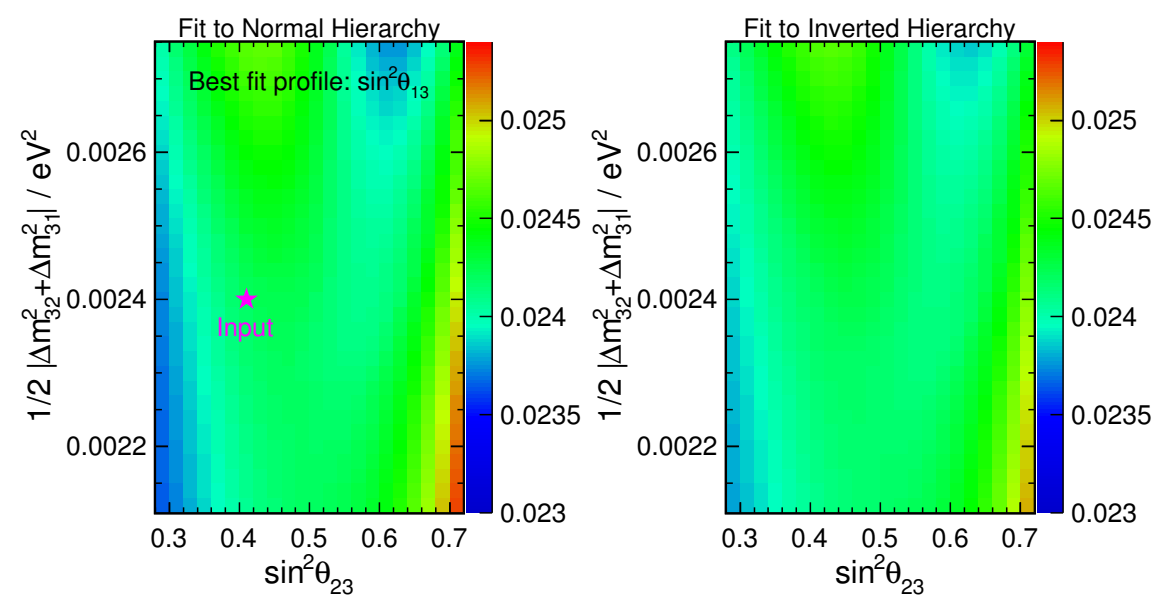

Figure 6.8: The best fit to $\sin ^{2}\left(\theta_{13}\right)$ as a function of $\theta_{23}$ and $\Delta m_{\text {atm }}^{2}$ for the normal hiearchy on the left and the inverted on the right.
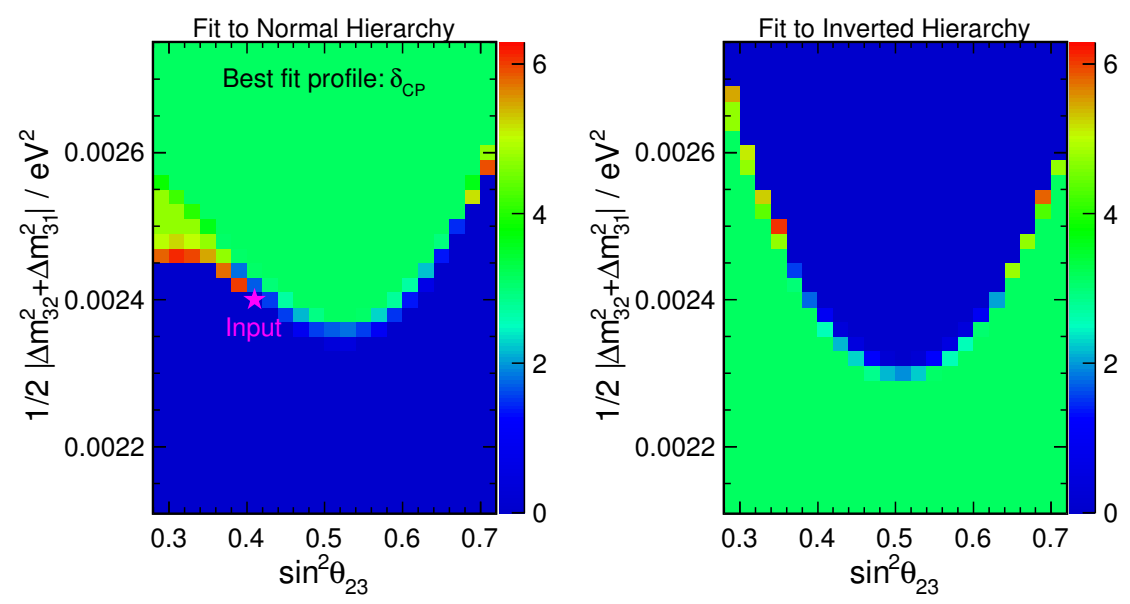

Figure 6.9: The best fit of $\delta_{c p}$ as a function of $\theta_{23}$ and $\Delta m_{a t m}^{2}$ for the normal hiearchy on the left and the inverted on the right.

The relatively large effect of $\delta_{c p}$ can be best understood if we expand our effective mass splitting to first order in $\cos \delta_{c p}$ with a none zero $\theta_{13}$ (without 
matter effects):

$$
\Delta m_{\text {atm }}^{2}=\Delta m_{32}^{2}+\Delta m_{21}^{2} \sin ^{2} \theta_{12}+\Delta m_{21}^{2} \cos \delta_{c p} \sin \theta_{13} \tan \theta_{23} \sin 2 \theta_{12}
$$

which shows that even in a vacuum we can expect the muon disappearance probability to have some dependance on $\delta_{c p}$.

This has motivated us to include $\theta_{13}$ as a nuisance parameter and $\delta_{c p}$ as a free parameter in the fit and to fix $\theta_{12}$ and $\Delta m_{21}^{2}$. The lack of dependance of $\theta_{13}$ on the atmospheric mixing parameters also tells us that any correlation between MINOS and the reactor experiments (which use our measurement of $\Delta m_{\text {atm }}^{2}$ ) is very weak. Values of $\Delta m_{21}^{2}=7.54 \times 10^{-5} \mathrm{eV}^{2}$ and $\sin ^{2}\left(\theta_{12}\right)=0.307$ were taken from the 2012 Fogli global analysis 48 . We use a $\sin ^{2}\left(\theta_{13}\right)$ which is a combination of the measurements found in Table 6.1, where systematic and statistical errors have been combined in quadrature.

Following the PDG 49] prescription for combining multiple measurements:

$$
\bar{x} \pm \delta \bar{x}=\frac{\sum_{i} w_{i} x_{i}}{\sum_{i} w_{i}} \pm \frac{1}{\sqrt{\sum_{i} w_{i}}}
$$

where $\bar{x}$ is the final best fit value, $\delta \bar{x}$ is its uncertainty, $x_{i}$ is the best fit of the $i^{\text {th }}$ input value, $\delta x_{i}$ is the uncertainty of the $i^{t h}$ input value and $w_{i}=\frac{1}{\left(\delta x_{i}\right)^{2}}$. We arrive at $\sin ^{2}\left(2 \theta_{13}\right)=0.094 \pm 0.010$ or $\sin ^{2}\left(\theta_{13}\right)=0.0242 \pm 0.025$.

\subsection{Result}

The three flavour analysis uses the same analysis methods and data sets as the previously described CC analysis, and the same general method for the final fit as 
described in Section 5.3 with the changes to oscillation parameters fitted as described above. The final fit result is shown in Table 6.2 and includes the best fits for each mass hiearchy and $\sin ^{2}\left(\theta_{23}\right)$ octant combination. Figures 6.10 and 6.11 show the constraints on $\sin ^{2}\left(\theta_{23}\right)$ and $\Delta m_{32}^{2}$ in each mass hiearchy. We observe a very mild preference for the inverted mass hiearchy and no ability to distinguish the octants.

\begin{tabular}{|l|c|c|}
\hline Experiment & $\sin ^{2}\left(2 \theta_{13}\right)$ & $\sin ^{2}\left(2 \theta_{13}\right)$ combined error \\
\hline Daya Bay & $0.089 \pm 0.010 \pm 0.005$ & $0.089 \pm 0.011$ \\
Reno & $0.113 \pm 0.013 \pm 0.019$ & $0.113 \pm 0.023$ \\
Double Chooz & $0.109 \pm 0.030 \pm 0.025$ & $0.109 \pm 0.039$ \\
\hline
\end{tabular}

Table 6.1: Three leading measurements of $\sin ^{2}\left(\theta_{13}\right)$ from Day Bay [52], Reno[107], and Double Chooz[108]. 

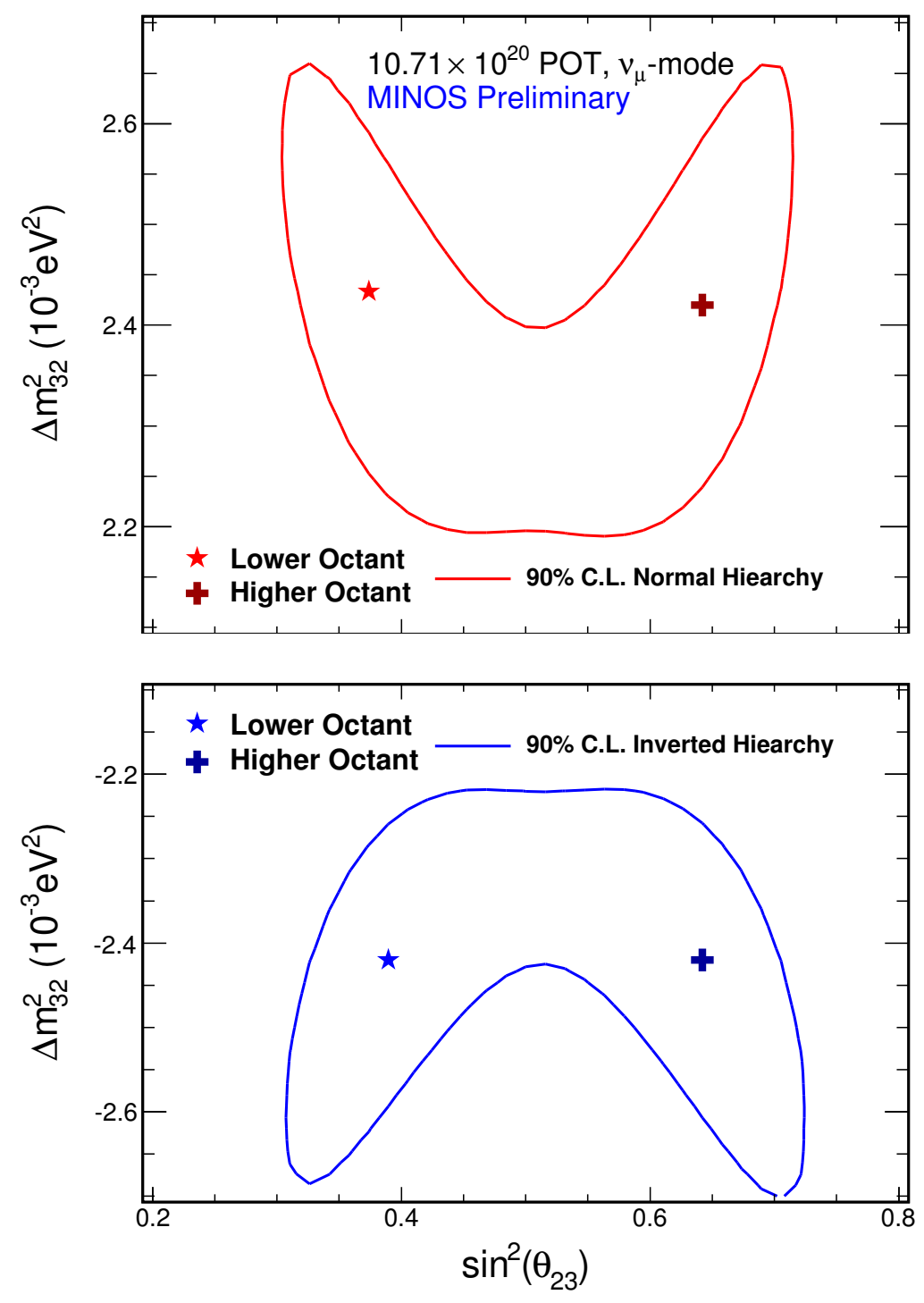

Figure 6.10: 90\% C.L. for both normal and inverted hiearchy with best fit points for the lowest $\log (L)$ in each combination of octant and hiearchy.

\begin{tabular}{|l|c|c|c|c|c|}
\hline Hiearchy and & \multicolumn{4}{|c|}{ Best Fit Oscillation Parameters } & $\Delta \chi^{2}=$ \\
Octant & $\sin ^{2}\left(\theta_{23}\right)$ & $\Delta m_{31}^{2}\left(10^{-3} \mathrm{eV}^{2}\right)$ & $\sin ^{2}\left(\theta_{13}\right)$ & $\delta_{c p} / \pi$ & $-2 \Delta \log (L)$ \\
\hline Normal, Lower & 0.38 & 2.44 & 0.0242 & 1.83 & 0.01 \\
Normal, Upper & 0.64 & 2.42 & 0.0242 & 1.90 & 0.01 \\
Inverted, Lower & 0.38 & 2.42 & 0.0242 & 0 & 0 \\
Inverted, Upper & 0.64 & 2.42 & 0.0242 & 0 & 0 \\
\hline
\end{tabular}

Table 6.2: Best fits to the mixing parameters allowed to float in our fit for each combination of mass hiearchy and octant. 

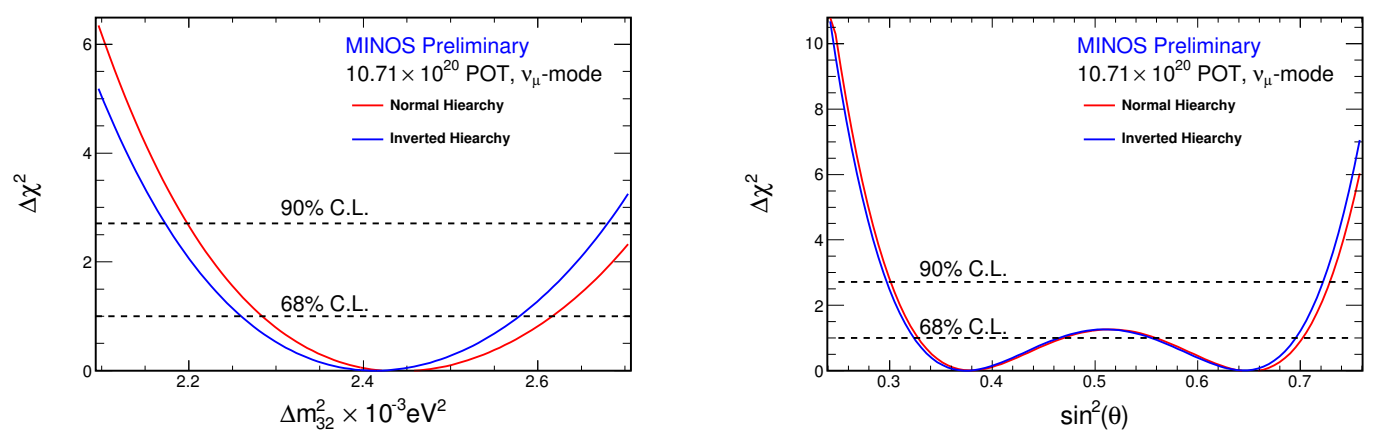

Figure 6.11: 1D marginalizations of our full contour for $\Delta \chi^{2}$ for $\Delta m_{32}^{2}$ (left) and $\sin ^{2} \theta_{23}$ (right). Show are both the normal (red) and inverted (blue) mass hiearchies. Dashed lines show $68 \%$ and 90\% C.L. 


\section{Chapter 7}

\section{Simulating the NuMI Beam}

The NuMI beam continues to be a centre of oscillation research, and comes out of the first stage of a planned upgrade to 700kw in August 2013[109]. In addition to the MINOS+[110] and $\mathrm{NO} \nu \mathrm{A}[109]$ experiments there have been suggestions for new potential experiments to study the NuMI beam. These experiments, such as CHIPS [11], seek to make complementary measurements of the atmospheric mixing parameters, the mass hierarchy, and $\delta_{c p}$.

To that end we need a tool for assessing the physics reach of different physical locations in the NuMI beam. We look in particular at the geographic region in northern Minnesota, referred to as the Mesabi Range, where the NuMI beam emerges from the earth after its long journey from FNAL.

The FLUGG simulation of the NuMI neutrino beam is discussed in some detail in Chapter 3 Section 3. By taking advantage of the fact that neutrino production from a decaying hadron is isotropic, and that the existing simulations store neutrino parent information, we can reweight the existing $\mathrm{MC}$ to give a neutrino flux representative of any hypothetical location[112. Furthermore, we scan over 
a region of interest, such as the Mesabi range, and construct a map of flux characteristics such as peak energies. Cross section and oscillation information can also be included so as to give a clear, intuitive, impression of the oscillation sensitivity at a given location.

\subsection{Flux Prediction}

\subsubsection{Coordinate Transformations}

Before we can reweight our MC we need to know the location of our hypothetical detector in the NuMI beam MC reference frame, and as a search for a new detector location is likely very sensitive to which real world locations are most convenient we would ideally like a system for converting from a set of Global Positioning System Coordinates and heights above sea level. The first step in any such conversion is the transformation from GPS coordinates to an earth centred Cartesian frame. This is achieved here by assuming the WGS84 ellipsoid as our model of the earth, a reasonable assumption as most modern GPS systems will convert to the WGS84 convention before giving you a location. The eccentricity of this ellipsoid is then:

$$
e c c=\sqrt{\frac{(a \times a)-(b \times b)}{a \times a}}
$$

where, for the WGS84 ellipsoid, $a=6378.137$ and $b=6356.7523141$. For longitude $\theta$, latitude $\phi$, and height above sea level, $H$, the earth centred Cartesian coordinates are then:

$$
\begin{gathered}
x=(v+\phi) \cos \phi \cos \theta \\
y=(v+\phi) \cos \phi \sin \theta \\
z=\left(\left(1-e^{2}\right) v+H\right) \sin \phi
\end{gathered}
$$


where $v$ is:

$$
v=\frac{a}{\sqrt{1-e^{2} \sin ^{2} \phi}} .
$$

Once we have our earth centred frame we just need a translation and rotation matrix to arrive at the NuMI MC coordinate system, taking the form of a standard Helmert transformation:

$$
X_{T}=C+R X
$$

where $X_{T}$ is the final vector coordinate, $X$ the earth centred coordinate, and $R$ and $C$ are the rotation and translation matrices defined below:

$$
\begin{gathered}
R=\left(\begin{array}{ccc}
\cos \theta_{z} \cos \theta_{y} & \cos \theta_{z} \sin \theta_{y} \sin \theta_{x}-\sin \theta_{z} \cos \theta_{x} & \cos \theta_{z} \sin \theta_{y} \cos \theta_{x}+\sin \theta_{z} \sin \theta_{x} \\
\sin \theta_{z} \cos \theta_{y} & \sin \theta_{z} \sin \theta_{y} \sin \theta_{x}+\cos \theta_{z} \cos \theta_{x} & \sin \theta_{z} \sin \theta_{y} \cos \theta_{x}-\cos \theta_{z} \sin \theta_{x} \\
-\sin \theta_{y} & \cos \theta_{y} \sin \theta_{x} & \cos \theta_{y} \cos \theta_{x}
\end{array}\right) \\
C=\left(\begin{array}{ccc}
D x & D x & D y
\end{array}\right)
\end{gathered}
$$

where, $D x=-8208.34535, D y=-6356836.852, D z=390470.2363, \theta_{x}=$ 44.928487, $\theta_{y}=25.093597$, and $\theta_{z}=-179.85961$.

\subsection{Reweighting}

With the location now in beam coordinates we can use parent momentum information to create an approximation of the neutrino flux at that point. As neutrino production from a decaying pion or kaon is isotropic we can draw a solid angle between the decaying hadron and the detector in the hadrons rest frame the size of which will be directly proportional to the probability of a neutrino from that hadron passing through the detector. Likewise, the angle of production is directly related to the energy of the produced neutrino so the energy of a neutrino which would pass through the detector can also be estimated. 


\subsubsection{Neutrino Energy Estimation}

Starting with a hadronic decay in the hadronic rest frame with the hadron's four momentum $P_{h}^{\prime}$, muon's four momentum $P_{\mu}^{\prime}$, and neutrino's four momentum $P_{\nu}^{\prime}$ :

$$
\begin{aligned}
& P_{h}^{\prime}=P_{\mu}^{\prime}+P_{\nu}^{\prime} \\
& P_{h}^{\prime}-P_{\nu}^{\prime}=P_{\mu}^{\prime}
\end{aligned}
$$

$P_{h}^{\prime}$ in its rest frame is just its mass $m_{h}$ such that if we take the dot product of each side of the equations with itself:

$$
\begin{gathered}
m_{h}^{2}+2\left(E_{\nu}^{\prime} m_{h}-0\right)=m_{\mu}^{2} \\
E_{\nu}^{\prime}=\frac{m_{\mu}^{2}-m_{h}^{2}}{2 m_{h}}
\end{gathered}
$$

where $m_{\mu}$ is the muon mass and $E_{\nu}^{\prime}$ is the energy of the neutrino in the hadronic rest frame. Back in the lab frame where the hadron's three momentum $p_{h}$, hadron decay point $r_{h}$, and neutrino's direction of flight $r_{\nu}$, the angle $\theta$ between the hadron and neutrino direction of flight is:

$$
\cos \theta=\frac{\left(r_{h}-r \nu\right) \cdot p_{h}}{\left|r_{h}-r_{\nu}\right|\left|p_{h}\right|}
$$

The neutrino energy in the lab frame $E_{\nu}$ as a function of $E_{\nu}^{\prime}$ and $\cos \theta$ can then be found:

$$
\begin{gathered}
P_{h}^{\prime} \cdot P_{\nu}^{\prime}=P_{h} \cdot P_{\nu} \\
E_{h}^{\prime} E_{\nu}^{\prime}-p_{h}^{\prime} \cdot p_{\nu}^{\prime}=E_{h} E_{\nu}-p_{h} \cdot p_{\nu} \\
m_{h} E_{\nu}^{\prime}-0=E_{h} E_{\nu}-\left|p_{h}\right|\left|p_{\nu}\right| \cos \theta \\
E_{\nu}=\frac{m_{h} E^{\prime} \nu}{E_{h}-\left|p_{h}\right| \cos \theta} \\
E_{\nu}=\frac{E_{\nu}^{\prime}}{\gamma_{h}\left(1-\beta_{h} \cos \theta\right)}
\end{gathered}
$$




\subsubsection{Decay Probability}

Now that we have the neutrino energy as function of $\theta$ we need to calculate the probability of the decay at that angle occurring. The probability is related to the angular distribution of parent decays which for a hadron is isotropic such that:

$$
\frac{d N}{d \cos \theta^{\prime}}=\frac{1}{2}
$$

The situation is somewhat more complicated for muon decays as the muon is a spin half particle such that $\frac{d N}{d \cos \theta^{\prime}} \neq \frac{1}{2}$. The muon itself is the product of a pion or kaon decay, and hence to conserve angular momentum its spin in its rest frame must be opposite to the direction of the incoming hadron. The angular distribution of decays as a function of the angle $\theta_{S}^{\prime}$ between the muon spin and momentum of the muon neutrino produced in the muon decay:

$$
\frac{d N_{\mu^{ \pm}}}{d \cos \theta_{S}^{\prime}}=\frac{1}{2}\left(1 \mp \frac{1-2 x}{3-2 x} \cos \theta_{S}^{\prime}\right)
$$

where $x=\frac{E_{\nu \mu}}{E_{\nu_{\mu}}^{\max }}, E_{\nu_{\mu}}$ is the muon neutrino energy, and $E_{\nu_{\mu}}^{\max }$ is the maximum possible neutrino energy $\frac{m_{\mu}}{2}$. However we know that $\cos \theta_{\pi}^{\prime}= \pm \cos \theta_{S}^{\prime}$ so we can instead write the angular distribution as a function of $\theta_{\pi}^{\prime}$ :

$$
\frac{d N_{\mu \pm}}{d \cos \theta_{\pi}^{\prime}}=\frac{1}{2}\left(1 \mp \frac{1-2 x}{3-2 x} \cos \theta_{\pi}^{\prime}\right)
$$

The final step is then to translate our angular distributions to the lab frame, making use of:

$$
\frac{d N}{d \cos \theta}=\frac{d N}{d \cos \theta^{\prime}} \frac{d \cos \theta^{\prime}}{d \cos \theta}
$$

and the $\theta$ dependence of the transverse momentum of the muon neutrino $p_{\|}$:

$$
\begin{aligned}
& \cos \theta=\frac{p_{\|}}{E_{\nu}} \\
& \cos \theta^{\prime}=\frac{p_{\|}^{\prime}}{E_{\nu}^{\prime}}
\end{aligned}
$$


$p_{\|}^{\prime}$ and $E_{\nu}^{\prime}$ can then be related to the lab frame by a Lorentz transformation:

$$
\begin{aligned}
& p_{\|}^{\prime}=\gamma_{h}\left(p_{\|}-\beta_{h} E_{\nu}\right) \\
& E_{\nu}^{\prime}=\gamma_{h}\left(E_{\nu}-\beta_{h} p_{\|}\right)
\end{aligned}
$$

Such that:

$$
\begin{aligned}
\cos \theta^{\prime} & =\frac{p_{\|}-\beta_{h} E_{\nu}}{E_{\nu}-\beta_{h} p_{\|}}=\frac{\cos \theta-\beta_{h}}{1-\beta_{h} \cos \theta} \\
\frac{d \cos \theta^{\prime}}{d \cos \theta}= & \frac{1}{1-\beta_{h} \cos \theta}+\beta_{h} \frac{\cos \theta-\beta_{h}}{\left(1-\beta_{h} \cos \theta\right)^{2}} \\
& =\frac{1-\beta_{h}^{2}}{\left(1-\beta_{h} \cos \theta\right)^{2}} \\
& =\frac{1}{\gamma^{2}} \frac{1}{\left(1-\beta_{h} \cos \theta\right)^{2}}
\end{aligned}
$$

Which gives us, for hadronic decays:

$$
\frac{d N}{d \cos \theta}=\frac{1}{2 \gamma^{2}\left(1-\beta_{h} \cos \theta\right)^{2}}
$$

and for muon decays:

$$
\frac{d N_{\mu \pm}}{d \cos \theta}=\left(1 \mp \frac{1-2 x}{3-2 x} \cos \theta_{\pi}^{\prime}\right) \frac{1}{2 \gamma^{2}\left(1-\beta_{h} \cos \theta\right)^{2}}
$$

Finally, the probability of a parent hadron decaying to produce a neutrino that passes through some hypothetical detector of surface area $D_{A}$ is given by the integral of the solid angle subtended by $D_{A}$ at the decay point:

$$
\begin{gathered}
P\left(\nu_{D_{A}}\right)=\int_{D_{A}} \frac{1}{4 \pi} \frac{d N}{d \cos \theta} d \Omega \\
=\frac{1}{4 \pi} \frac{d N}{d \cos \theta_{D_{A}}} \Omega_{D_{A}}
\end{gathered}
$$




\subsection{Predicted Spectrum}

Now that we have the tools for finding the NuMI beam coordinates of any GPS location and those for reweighting a neutrino event in our MC for any NuMI coordinate we can create an estimate of the beam flux seen at both well studied detector locations (for the sake of validation of the technique) and hypothetical detector locations. One such detector location is the Wentworth pit, potential home of the CHIPS detector[111, in northern Minnesota. Figure 7.1 shows the muon neutrino flux there in comparison to the same beam exposure at MINOS and $\mathrm{NO} \nu \mathrm{A}[109]$.

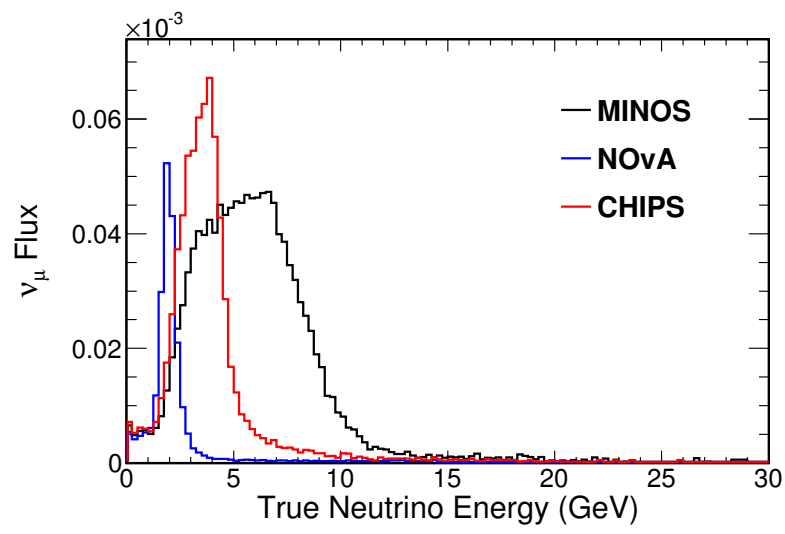

Figure 7.1: Neutrino flux as a function of energy at the MINOS, NO $\nu$ A, and CHIPS Far Detector locations assuming null oscillations.

\subsubsection{Cross Sections and Neutrino Oscillations}

We do not directly measure the muon neutrino flux, and we certainly do not measure it without neutrino oscillations. In order to produced a simple and intuitive tool for assessing the use of a potential site for the study of three flavour neutrino oscillations we are interested in the spectrum of charge current interactions before and after neutrino oscillations. Cross sections were estimated using a cubic spline of the energy dependant CC cross sections on iron (taken from GENIE, 
see Figure 7.2 and normalizing to one kiloton so as to give a good estimate of the event rate on one kiloton of any material (subtle differences in neutrino cross sections between materials are not important for this sort of qualitative study). Neutrino oscillations meanwhile were calculated using the same full three flavour treatment of the PMNS as the MINOS three flavour analysis, Chapter 6, calculating the oscillation probability (after reweighting) of every neutrino event in the beam MC. Figure 7.3 shows the muon neutrino charge current interaction spectrum at the Wentworth Pit in comparison to MINOS and $\mathrm{NO} \nu \mathrm{A}$, and the event rate before and after oscillations at the Wentworth Pit.

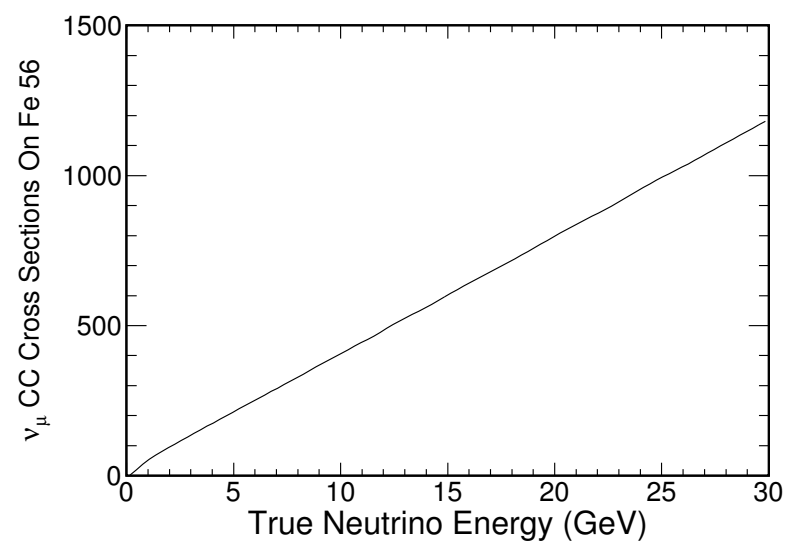

Figure 7.2: A spline of CC cross sections as a function of energy.

\subsubsection{Peak Finding}

The ROOT analysis package contains a spectrum package with a sophisticated peak finder which makes use of a reverse Markov chain algorithm to look for peaks in a histogram. This tool creates a highly accurate estimate of the peak energy of neutrino spectrum, such as those in Figure 7.3. This estimate is a powerful tool when mapping the characteristics of the neutrino beam across a large geographic area as the L/E tells you how close you are to an oscillation maximum. Figure 7.4 shows the peak finding tool being used on an example spectrum. 

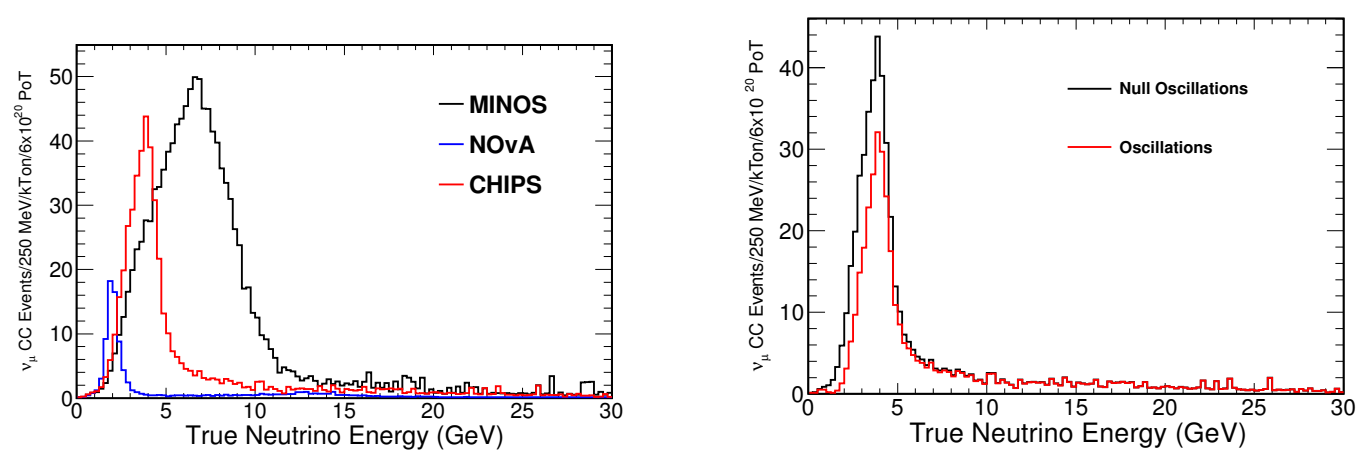

Figure 7.3: On the left are comparative muon neutrino true energy spectrums distributions at the MINOS, NO $\nu$ A, and CHIPS Far Detector locations assuming null oscillations and one kiloton-year of running. On the right is the true muon neutrino event spectrum that would be seen at CHIPS in one kiloton-year with (red) and without (black) neutrino oscillations.

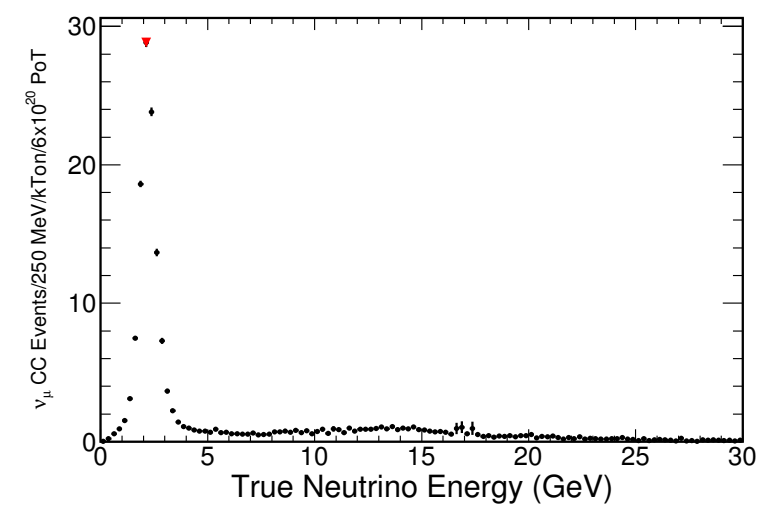

Figure 7.4: A spectrum for a hypothetical detector location in the Mesabi range(black line) with the peak estimation shown (red dot).

\subsection{Mapping the Beam}

With a series of tools capable of producing neutrino flux, events rates, and calculating peak energy for any GPS location it is a simple extension to use them to create an overlay of information about the neutrino beam at a given location over a map of the same area. Figure 7.5 shows the muon neutrino CC event rate for one kiloton year in the case of null oscillation in the area of northern Minnesota 
known as the Mesabi Range, also shown are lines of constant peak L/E so that in combination you can estimate both your event rate and your sensitivity to neutrino oscillations.

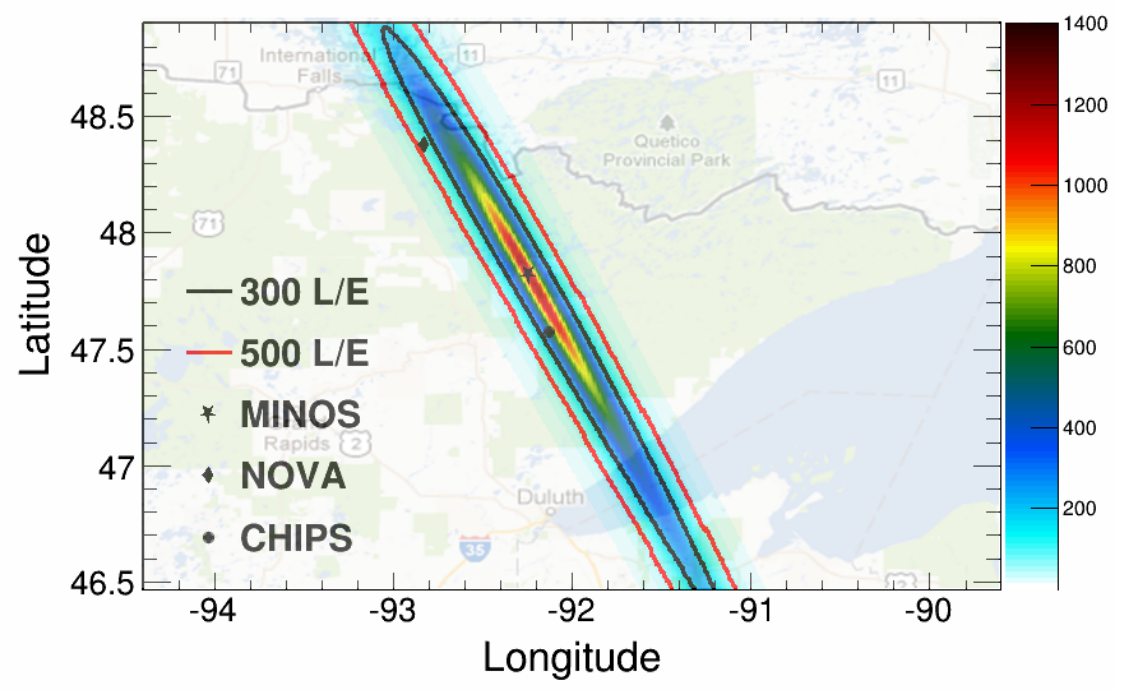

Figure 7.5: A map of potential neutrino event rates, assuming a null oscillations hypothesis, in the $0-30 \mathrm{GeV}$ region as seen within the Mesabi range within one Kiloton year. Contours show lines of constant L/E where $\mathrm{L}$ is the distance from the hypothetical detector to the NuMI target and E is the peak energy of the reweighted neutrino spectrum. 


\section{Chapter 8}

\section{Summary}

The world's knowledge of the oscillation mixing parameters is a very different landscape to the one MINOS first entered in 2006. Not only do we now have strong constraints on the solar and atmospheric mixing parameters, reactor experiments have shown us that $\theta_{13}$ is larger than we had dared hope.

With a strong constraint on a relatively large $\theta_{13}$ the challenge is now to measure

the octant of $\theta_{23}, \delta_{c p}$, and the mass hierarchy. Resolution of the $\theta_{23}$ and the question of whether or not it is maximal will be important in resolving the latter two questions. Particularly as the size of $\theta_{23}$ can effect the projected sensitivity and hence design of future experiments. The mass hierarchy will inform us about the prospects for any measurements of majorana neutrinos. A definitive measure of nonzero $\delta_{c p}$ in combination with a non zero majorana phase could answer the question of why we live in a matter dominated universe.

MINOS itself has lead the charge in the world's understanding of the atmospheric mass splitting in the last half decade, and it has helped to develop the methods and techniques that new neutrino experiments are just beginning to use. Detailed in this thesis (Chapters 5-6) is an update to the MINOS beam analysis 
which helped make possible the contour in Figure 8.1, a fit to the mixing parameters which makes full use of the MINOS NuMI beam disappearance, appearance, and atmospheric disappearance datasets. Moving forward the next generation of long baseline experiments, such as T2K, will follow a similar approach to maximise the constraints on mass hierarchy, and $\delta_{c p}$ that they can achieve [113]. The MINOS constraint on $\Delta\left|m_{\text {atm }}^{2}\right|$ continues to be used by reactor experiments, such as Daya Bay[52], in their precision measurement of $\theta_{13}$.

The NuMI beam continues to be a centre of oscillation research, and comes out of the first stage of a planned upgrade to 700kw in August 2013[109]. MINOS+[110], $\mathrm{NO} \nu \mathrm{A}[109$, and potential new experiments such as CHIPS [111] will make use of this new high intensity beam to push on the the worlds knowledge of the atmospheric mixing parameters, the mass hierarchy and $\delta_{c p}$. The longer, $810 \mathrm{~km}$, baseline of $\mathrm{NO} \nu \mathrm{A}$ will be particularly important in the resolution of the mass hierarchy where matter effects play the crucial role described in Chapter 2.5.3 of this thesis. Chapter 7 of this thesis describes a study into the sensitivity to neutrino oscillations in this new NuMI beam at different locations in the Mesabi range.

Aside from the questions asked of us by the current formulation of the PMNS matrix, there are others. The search for sterile neutrinos, described in Chapter 2.7.4 of this thesis continues with new experiments such as Microboone[114] and MINOS+. A search which could offer an answer to the question of where the majority of the cosmos's mass is hiding. Extensions to our current model of matter effects through non standard interactions continue as long baseline oscillation experiments provide increasingly precise measures of oscillation probabilities in matter. Even searches for large extra dimensions are being conducted using data 
from neutrino oscillation experiments[115].

The future of our field is a bright one. Many excellent measurements have already been made. Many more remain. Perhaps even some which we have yet to think of. 


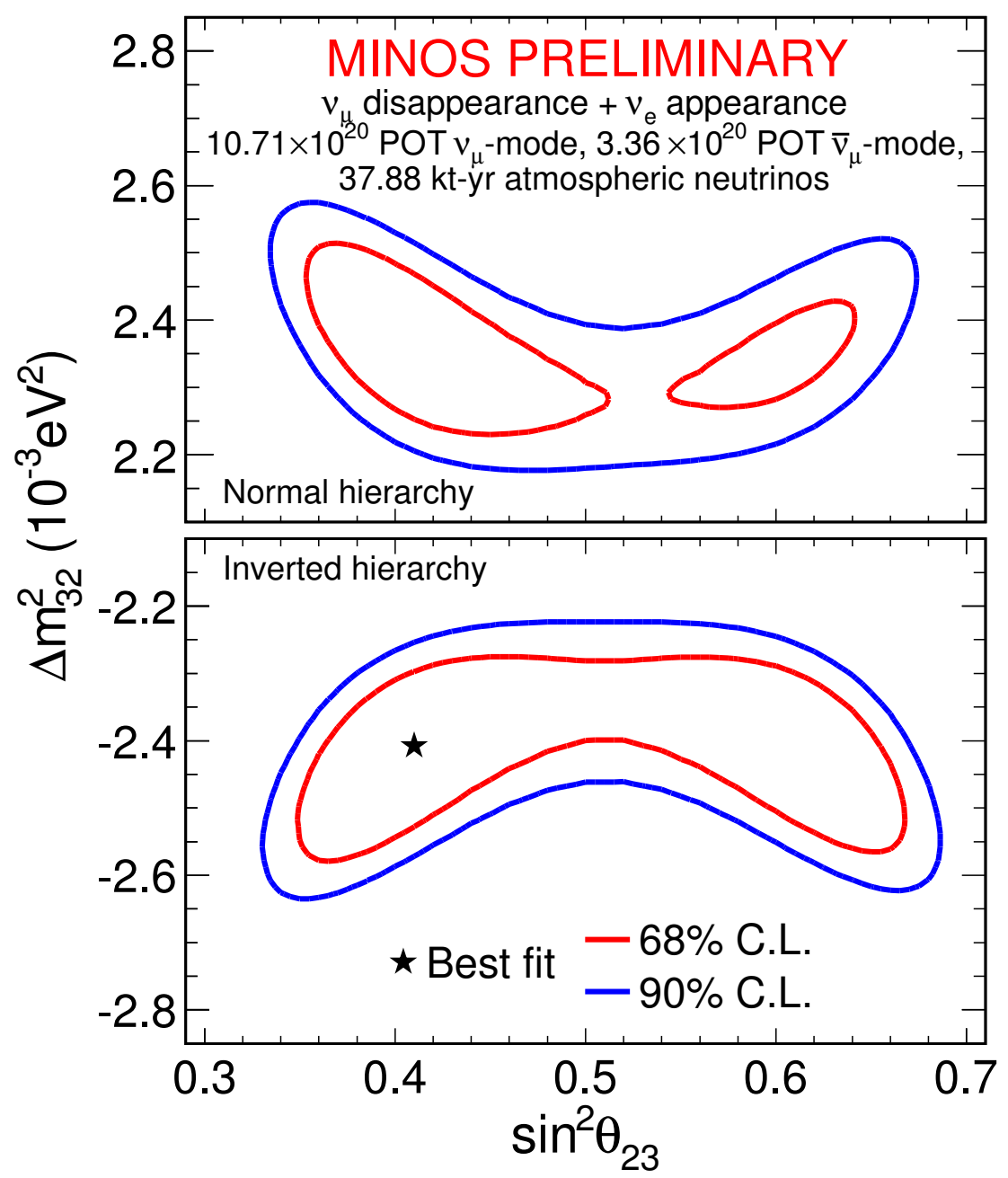

Figure 8.1: The $68 \%$ and $90 \%$ confidence limits for the $\left(\Delta m_{32}^{2}, \sin ^{2} \theta_{23}\right)$ parameter space, resulting from a combined fit to the MINOS $\nu_{\mu}$ disappearance and $\nu_{e}$ appearance data. The best fit occurs in the inverted hierarchy and lower octant at $\left(\Delta m_{32}^{2} / \mathrm{eV}^{2}, \sin ^{2} \theta_{23}\right)=\left(-2.41 \times 10^{-3}, 0.41\right)$, as indicated by the star. For both the normal and inverted hierarchy, the $-\Delta \log L$ surface is calculated relative to this overall best fit point. Figure take from [116]. 


\section{Chapter 9}

\section{Appendix}

\subsection{Deriving Matter Density in the NuMI Beam}

As we now use the exact three flavour neutrino oscillation model with matter effects, we must make a choice about the density of matter between Fermilab and the Soudan Mine in Minnesota. It has already been shown by the MINOS UP group [106] that even for long baselines they are largely insensitive to uncertainties in the matter density. This will be used as a justification for the approximations used in this derivation.

\subsubsection{Average Depth of the NuMI Beam}

Whilst the earth must be treated as an ellipsoid over large distances, and this ellipsoid is well described, we have chosen to use the approximation of a circle for this problem so as to make use of several well known trigonometric properties. The first of which is to think of the NuMI beam as a chord cutting through the the earth: 


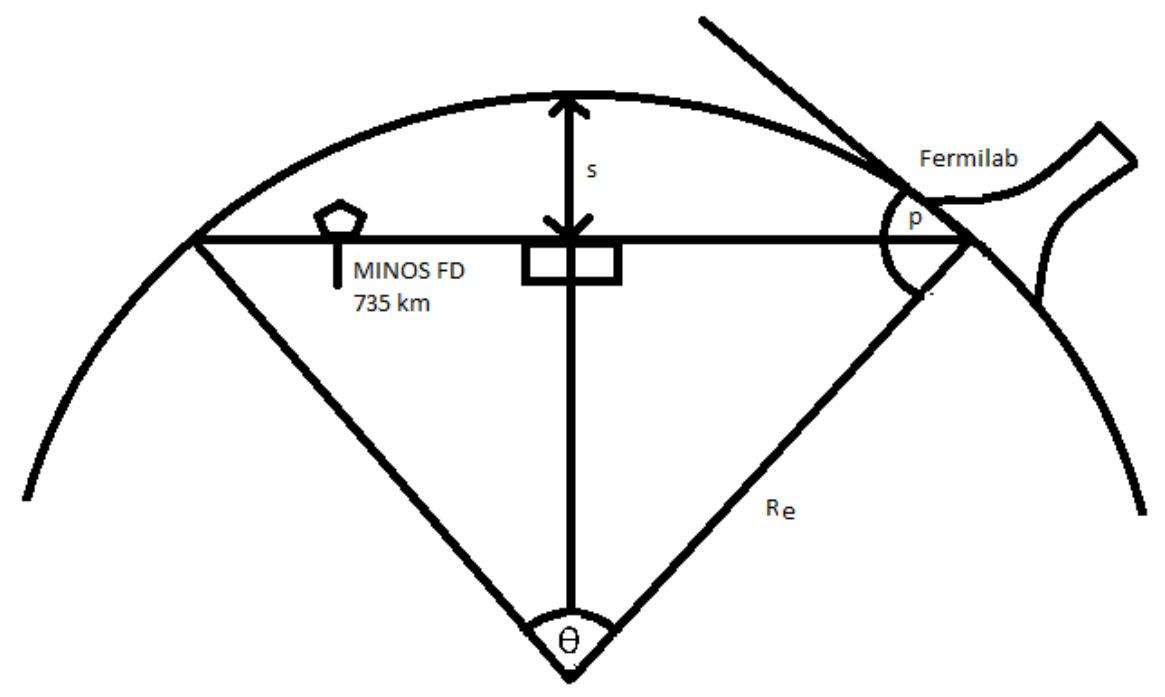

Figure 9.1: A diagram showing the simplified NuMI geometry we are about use.

Now if we approximate the NuMI beam as starting on the surface of the earth and take advantage of the known pitch of our beam of 3.34016 degrees [60] we have a well defined chord of length:

$$
C_{L}=2 \sin \left(\frac{\theta}{2}\right)=742 \mathrm{~km}
$$

which allows us, for any point along the beamline (x), to have a well defined depth $(D(x))$, assuming and earth of radius $6371 \mathrm{~km}\left(R_{e}\right)$ :

$$
D(x)=R_{e}-\sqrt{\left(R_{e}^{2}-x^{2}-C_{L} x\right)}
$$

- We can then calculate the mean depth using a computatial package such as Mathematica to perform a numerical integration in terms of $x$ over the MINOS baseline and then divide by the baseline:

$$
\int_{1.04}^{735} \frac{D(x)}{735-1.04}=7.28 \mathrm{~km}
$$

And hence we can calculate an average $\mathrm{D}(\mathrm{x})$ over the NuMI baseline of $7.28 \mathrm{~km}$. 


\subsubsection{The Density of the Crust}

The CRUST 2.0 global crustal model provides estimates of density as a function of the radius out from the centre of the earth. Using the calculated average depth of $7.28 \mathrm{~km}$ this gives us a density of $2.76 \mathrm{~g} / \mathrm{cm}^{3}$ or an electron density of $N_{e^{-}}=1.36$ moles $/ \mathrm{cm}^{3}$ (assuming a Z/A in the earths crust of 0.494). 


\subsection{Summary of Personal Contributions}

Since starting graduate studies at University College London in 2010 I have worked on the MINOS experiment, with a strong focus on measurements of muon neutrino disappearance in the NuMI beam. Initially this took the form of assisting the 2011 anti-neutrino analysis at MINOS, where I carried out numerous background, reconstruction and systematics studies in preparation for the box opening. I took over the task of monitoring the MINOS data quality by making validation plots from our Near Detector data for all MINOS analysis groups that use NuMI beam data. During this time I became familiar with the FLUGG MC package and developed a framework for MINOS beam flux file production using the fermilab computing grid.

During 2012 I worked as lead researcher on two flavour NuMI Beam Muon Neutrino Disappearance studies at MINOS where I updated the analysis cuts and validated new datasets used in the analysis. I produced the updated MINOS beam muon neutrino disappearance measurement of $\sin ^{2}\left(2 \theta_{23}\right)$ and $\Delta\left|m_{a t m}^{2}\right|$. This measurement was used as part of the full MINOS beam and atmospheric neutrino disappearance analysis to give the worlds leading measurement of $\Delta\left|m_{\text {atm }}^{2}\right|$, first shown at Neutrino 2012. As part of this analysis role I have been heavily involved in the MINOS disappearance analysis PRD, which is currently aiming for publication this summer.

More recently I have worked as lead researcher on the update of the NuMI Beam Muon Neutrino Disappearance analysis to a full three flavour treatment. I updated the core software framework, changed the way oscillations are applied, and investigated the correct choice of parameters in a three flavour Muon Neutrino Disappearance fit. This work was used as part of the full MINOS combined beam 
and atmospheric neutrino appearance and disappearance analysis presented during the summer of 2013.

In addition to my work for the MINOS Disappearance group I have produced a series of maps of neutrino event and flux maps in the NuMI beam for the NuMI Exploitation Working Group. This involved producing a tool for generating both oscillated and unoscillated neutrino event spectra and flux in the NuMI beam for any set of GPS coordinates and height above sea level. I then extended this same tool to create maps of neutrino event rates and flux in the area covered by the NuMI beam. This work was used as the basis for a section of a whitepaper suggesting a new water cherenkov detector in the NuMI beam called CHIPS. 


\section{Bibliography}

[1] A. Einstein. Uber Einen die Erzeugung und Verwandlung des Lichtes betreffenden heuristischen Gesichtspunkt. Ann. Phys., 322:132148, 1905.

[2] J. Chadwick. Distribution in intensity in the magnetic spectrum of the $\beta$-rays of radium. Deutch. Phys. Gessel., 16. 8.:883-891, 1914.

[3] C. D. Ellis and W. A Wooster. The Average Energy of Disintegration of Radium E. Proceedings of the Royal Society of London, 117:A, 117(776):109123, 1927.

[4] Niels Bohr. Faraday Lecture. 8th of May 1930.

[5] Wolfgang Pauli. Letter to the physical society of Tubingen. 1930.

[6] J. Chadwick. Possible Existence of a Neutron. Nature, 129:312, 1932.

[7] Enrico Fermi. Tentativo di una teoria dei raggi $\beta$. Ricerca Scientifica, 1933.

[8] Fred Wilson. Fermi's Theory of Beta Decay. American Journal of Physics, 36:1150-1160, 1968.

[9] H. A. Bethe and R. Peierls. The Scattering og Neutrons by Protons. 1934.

[10] C.L. Cowan F. Reines. Detection of the free neutrino. Phys. Rev., 92:830831, 1953. 
[11] F. Reines F. B. Harrison H. W. Kruse A. D. McGuire C. L. Cowan, Jr. Detection of the Free Neutrino: a Confirmation. Science, 124:103-104, 1956.

[12] W. Heisenberg. Zur Theorie der "Schauer" in der Hohenstrahlung. Zeitschrift fur Physik, 101:533, 1936.

[13] T.D. Lee and C.N. Yang. Question of Parity Conservation in Weak Interactions. Phys. Rev., 104:254, 1956.

[14] R.W. Hayward. D. D. Hoppes C.S. Wu, E. Ambler and R. P. Hudson. Experimental Test of Parity Conservation in Beta Decay. Phys. Rev., 105:1413.

[15] H. Yukawa. On the Interaction of Elementary Particles. Proc. Phys. Math. Soc. Japan, 17:48, 1935.

[16] R. P. Feynman and M. Gell-Man. Theory of the Fermi Interaction. Phys. Rev., 109:193, 1958.

[17] E.C. G. Sudarshan and R. R. Marshak. Chirality Invariance and the Universal Fermi Interaction. Phys. Rev., 109:1860, 1958.

[18] S. L. Glashow. Partial Symmetries of Weak Interactions. Nucl. Phys., 22:579, 1961.

[19] A. Salam and J. C. Ward. Partial Symmetries of Weak Interactions. Nucl. Phys., 22:579, 1964.

[20] S. Weinberg. A Model of Leptons. Phys. Rev. Lett., 19:1264, 1967.

[21] Gargamelle Neutrino Collaboration. Search for elastic muon neutrino electron scattering. Phys. Lett, B46:121, 1973. 
[22] Gargamelle Neutrino Collaboration. Observation of neutrino-like interactions without muon or electron in the Gargamelle neutrino experiment. Phys. Lett., B46:138, 1973.

[23] G. Arnison et al. Experimental Observation of Isolatd Large Transverse Energy Electrons With Associate Missing Energy at $\sqrt{s}=540 \mathrm{GeV}$. Phys. Lett, B122:103, 1983.

[24] R. Banner et al. Observation of Single Isolated Electrons of High Transverse Momentum in Events with Missing Transverse Energy at the CERN $\bar{p} p$ Collider. Phys. Lett., B122:476, 1983.

[25] G. Arnison et al. Experimental observation of lepton pairs of invariant mass around $95 \mathrm{GeV} /{ }^{2}$ at the CERN SPS Collider. Phys. Lett, B122:398, 1983.

[26] P.Bagnaia et al. Evidence for $Z^{0} \rightarrow e^{+} e^{-}$at the CERN anti-p p collider. Phys. Lett., B122:130, 1983.

[27] G. Danby, J.M. Gaillard, Konstantin A. Goulianos, L.M. Lederman, Nari B. Mistry, et al. Observation of High-Energy Neutrino Reactions and the Existence of Two Kinds of Neutrinos. Phys.Rev.Lett., 9:36-44, 1962.

[28] Martin L. Perl, G.S. Abrams, A. Boyarski, Martin Breidenbach, D. Briggs, et al. Evidence for Anomalous Lepton Production in e+ - e- Annihilation. Phys.Rev.Lett., 35:1489-1492, 1975.

[29] S. Schael et al. Precision electroweak measurements on the $Z$ resonance. Phys.Rept., 427:257-454, 2006.

[30] K. Kodama et al. Observation of tau neutrino interactions. Phys.Lett., B504:218-224, 2001. 
[31] K. Kodama et al. Final tau-neutrino results from the DONuT experiment. Phys.Rev., D78:052002, 2008.

[32] Raymond Davis, Don S. Harmer, and Kenneth C. Hoffman. Search for neutrinos from the sun. Phys. Rev. Lett., 20:1205-1209, May 1968.

[33] B.T. Cleveland, Timothy Daily, Jr. Davis, Raymond, James R. Distel, Kenneth Lande, et al. Measurement of the solar electron neutrino flux with the Homestake chlorine detector. Astrophys.J., 496:505-526, 1998.

[34] A.I. Abazov, O.L. Anosov, E.L. Faizov, V.N. Gavrin, A.V. Kalikhov, et al. Search for neutrinos from sun using the reaction Ga-71 (electron-neutrino e-) Ge-71. Phys.Rev.Lett., 67:3332-3335, 1991.

[35] P. Anselmann et al. Solar neutrinos observed by GALLEX at Gran Sasso. Phys.Lett., B285:376-389, 1992.

[36] John N. Bahcall, Aldo M. Serenelli, and Sarbani Basu. New solar opacities, abundances, helioseismology, and neutrino fluxes. Astrophys.J., 621:L85L88, 2005.

[37] B. Aharmim et al. Electron energy spectra, fluxes, and day-night asymmetries of B-8 solar neutrinos from measurements with $\mathrm{NaCl}$ dissolved in the heavy-water detector at the Sudbury Neutrino Observatory. Phys.Rev., C72:055502, 2005.

[38] K.S. Hirata et al. Observation of a small atmospheric muon-neutrino / electron-neutrino ratio in Kamiokande. Phys.Lett., B280:146-152, 1992.

[39] R. Becker-Szendy, C.B. Bratton, D. Casper, S.T. Dye, W. Gajewski, et al. The Electron-neutrino and muon-neutrino content of the atmospheric flux. Phys.Rev., D46:3720-3724, 1992. 
[40] Y. Ashie et al. Evidence for an oscillatory signature in atmospheric neutrino oscillation. Phys.Rev.Lett., 93:101801, 2004.

[41] W. Anthony Mann. Atmospheric neutrinos and the oscillations bonanza. Int.J.Mod.Phys., A15S1:29-256, 2000.

[42] B. Pontecorvo. Inverse beta processes and nonconservation of lepton charge. Sov.Phys.JETP, 7:172-173, 1958.

[43] B. Pontecorvo. Neutrino Experiments and the Problem of Conservation of Leptonic Charge. Sov.Phys.JETP, 26:984-988, 1968.

[44] Ziro Maki, Masami Nakagawa, and Shoichi Sakata. Remarks on the unified model of elementary particles. Prog.Theor.Phys., 28:870-880, 1962.

[45] L. Wolfenstein. Neutrino oscillations in matter. Phys. Rev. D, 17:23692374, May 1978.

[46] S.P. Mikheyev and A.Yu. Smirnov. Resonant amplification of oscillations in matter and solar-neutrino spectroscopy. Il Nuovo Cimento C, 9(1):17-26, 1986.

[47] S. Abe et al. Precision Measurement of Neutrino Oscillation Parameters with KamLAND. Phys.Rev.Lett., 100:221803, 2008.

[48] G.L. Fogli, E. Lisi, A. Marrone, D. Montanino, A. Palazzo, et al. Global analysis of neutrino masses, mixings and phases: entering the era of leptonic CP violation searches. Phys.Rev., D86:013012, 2012.

[49] J. et al Beringer. Review of particle physics. Phys. Rev. D, 86:010001, 2012.

[50] Yoshitaka Itow et al. Recent results in atmospheric neutrino oscillations in the light of large $\Theta_{13}$. Nucl.Phys.Proc.Suppl., 235-236:79-86. 
[51] P. Adamson et al. Measurement of Neutrino and Antineutrino Oscillations Using Beam and Atmospheric Data in MINOS. 2013.

[52] F.P. An et al. Improved Measurement of Electron Antineutrino Disappearance at Daya Bay. Chin. Phys., C37:011001, 2013.

[53] Francoise Gibrat-Debu Boris Kayser and Frederic Perrier. The physics of massive neutrinos. 1989.

[54] L.M. Ejzak. The Search for Neutrinoless Double Beta Decay in CUORE. 2009.

[55] A. Aguilar-Arevalo et al. Evidence for neutrino oscillations from the observation of anti-neutrino(electron) appearance in a anti-neutrino(muon) beam. Phys.Rev., D64:112007, 2001.

[56] A.A. Aguilar-Arevalo et al. Event Excess in the MiniBooNE Search for $\bar{\nu}_{\mu} \rightarrow \bar{\nu}_{e}$ Oscillations. Phys.Rev.Lett., 105:181801, 2010.

[57] P. Adamson et al. Electron neutrino and antineutrino appearance in the full MINOS data sample. Phys.Rev.Lett., 2013.

[58] P. Adamson et al. Active to sterile neutrino mixing limits from neutralcurrent interactions in MINOS. Phys.Rev.Lett., 107, 2011.

[59] Nathan Samuel Mayer. Measurement of the $M_{A}^{Q E}$ Parameter Using Multiple Quasi-Elastic Dominated Sub-Samples in the MINOS Near Detector. Phd. thesis, Indiana, 2011.

[60] J. Hylen et al. Conceptual design for the technical components of the neutrino beam for the Main Injector (NuMI). Fermilab-TM-2018, 1997.

[61] S. van der Meer. A directive device for charged particles and its use in an enhanced neutrino beam. 1961. 
[62] The Fermilab Main Injector Technical Design Handbook. 1994.

[63] S. Shukla, J. Marriner, and J. Griffin. Slip stacking in the Fermilab Main Injector. eConf, C960625:ACC015, 1996.

[64] C.C. Jensen and G.A. Krafczyk. NuMI proton kicker extraction system. Conf.Proc., C0505161:692, 2005.

[65] A.G. Abramov, N.A. Galyaev, V.I. Garkusha, J. Hylen, F.N. Novoskoltsev, et al. Beam optics and target conceptual designs for the NuMI project. Nucl.Instrum.Meth., A485:209-227, 2002.

[66] Alexander I. Himmel. The NuMI Beam Simulation with Flugg. MINOSdoc-6316, 2010.

[67] C. Alt et al. Inclusive production of charged pions in $\mathrm{p}+\mathrm{C}$ collisions at 158-GeV/c beam momentum. Eur.Phys.J., C49:897-917, 2007.

[68] I. Ambats et al. The MINOS Detectors Technical Design Report. 1998.

[69] D.G. Michael et al. The Magnetized steel and scintillator calorimeters of the MINOS experiment. Nucl.Instrum.Meth., A596:190-228, 2008.

[70] Pla-Dalmau A (MINOS Scintillator Group). Extruded Plastic Scintillator for the MINOS Calorimeters. Frascati Phys. Ser., 21:513522, 2001.

[71] K. Lang, J. Day, S. Eilerts, S. Fuqua, A. Guillen, M. Kordosky, M. Lang, J. Liu, W. Opaska, M. Proga, P. Vahle, A. Winbow, G. Drake, J. Thomas, C. Andreopoulos, N. Saoulidou, P. Stamoulis, G. Tzanakos, M. Zois, A. Weber, and D. Michael. Characterization of 1600 Hamamatsu 16-anode photomultipliers for the MINOS Far detector. Nuclear Instruments and Methods in Physics Research Section A: Accelerators, Spectrometers, Detectors and Associated Equipment, 545(3):852 - 871, 2005. 
[72] N. Tagg, A. De Santo, A. Weber, A. Cabrera, P.S. Miyagawa, et al. Performance of Hamamatsu 64-anode photomultipliers for use with wavelengthshifting optical fibres. Nucl.Instrum.Meth., A539:668-678, 2005.

[73] Richa Sharma. Position paper on the neutrino-antineutrino oscillations analysis in MINOS using 7.1e20 PoT neutrino and antineutrino dataset. MINOS-doc-9782, 2013.

[74] J. Oliver, N. Felt, G. Feldman, A. Lebedev, and R. Lee. Design and performance of the readout system of the MINOS Far Detector. IEEE Trans.Nucl.Sci., 51:2193-2195, 2004.

[75] T. Cundiff, J.W. Dawson, G. Drake, W. Haberichter, D. Reyna, et al. The MINOS near detector front end electronics. IEEE Trans.Nucl.Sci., 53:13471355, 2006.

[76] S. Avvakumov, W.L. Barrett, T. Belias, C. Bower, A. Erwin, et al. Spontaneous light emission from fibers in MINOS. Nucl.Instrum.Meth., A545:145$155,2005$.

[77] MINOS Calibration Group. 2011 Position Paper on Calibration of Runs I-VII. MINOS-doc-8263, 2011.

[78] P. Adamson et al. The MINOS light injection calibration system. Nucl.Instrum.Meth., A492:325-343, 2002.

[79] P. Adamson, G. Crone, L. Jenner, R. Nichol, R. Saakian, et al. The MINOS calibration detector. Nucl.Instrum.Meth., A556:119-133, 2006.

[80] R. Brun et al. Geant detector description and simulation tool. CERN Program Library Long Writeup, W5013, 1994. 
[81] M. Campanella, A. Ferrari, P.R. Sala, and S. Vanini. First Calorimeter Simulation with the FLUGG Prototype. 1999.

[82] M. Campanella, A. Ferrari, P.R. Sala, and S. Vanini. Reusing Code from FLUKA and GEANT4 Geometry. 1998.

[83] S. Agostinelli et al. Geant4 a simulation toolkit. Nuclear Instruments and Methods in Physics Research Section A: Accelerators, Spectrometers, Detectors and Associated Equipment, 506(3):250 - 303, 2003.

[84] F. Ballarini, G. Battistoni, F. Cerutti, A. Empl, A. Fasso, et al. Nuclear models in FLUKA: Present capabilities, open problems and future improvements. AIP Conf.Proc., 769:1197-1202, 2005.

[85] Mark Dorman. Beam fit position paper. MINOS-doc-7146, 2010.

[86] H. Gallagher. The NEUGEN Neutrino Event Generator. Nucl.Phys. Proc.Suppl., 112:188-194, 2002.

[87] Torbjorn Sjostrand, Stephen Mrenna, and Peter Z. Skands. PYTHIA 6.4 Physics and Manual. JHEP, 0605:026, 2006.

[88] Z. Koba, Holger Bech Nielsen, and P. Olesen. Scaling of multiplicity distributions in high-energy hadron collisions. Nucl.Phys., B40:317-334, 1972.

[89] T. Yang et al. A hadronization model for few-gev neutrino interactions. Eur. Phys. J., C63:1-10, 2009.

[90] R. Merenyi, W.A. Mann, T. Kafka, W. Leeson, B. Saitta, et al. Determination of pion intranuclear rescattering rates in muon-neutrino Ne versus muon-neutrino D interactions for the atmospheric neutrino flux. Phys.Rev., D45:743-751, 1992.

[91] G. Bishop G.Welch. An introduction to the kalman filter. TR 95-041, 2007. 
[92] H. Bethe. Zur Theorie des Durchgangs schneller Korpuskularstrahlen durch Materie. Annalen Phys., 397:325-400, 1930.

[93] Donald E. Groom et al. Muon stopping power and range tables $10 \mathrm{mev}$ to 100 tev. Atomic Data and Nuclear Data Tables, 78(2):183 - 356, 2001.

[94] Christopher James Backhouse. Measuring neutrino oscillation parameters using $\nu_{\mu}$ disappearance in MINOS. Phd. thesis, Oxford, 2011.

[95] MINOS Calibration Group. 2009 position paper on calibration of runs I-IIIII. MINOS-doc-6717, 2009.

[96] H. Gallagher M. Kordosky and S. Dytman. Shower energy scale uncertainty for the run I+II CC analysis. MINOS-doc-4287, 2009.

[97] Andy Blake. Blessed plots for the combined beam and atmospheric analysis. MINOS-doc-9172, 2012.

[98] Jessica Mitchell. Measuring $\nu_{\mu}$ Disappearance with the MINOS Experiment. Phd. thesis, Cambridge, 2011.

[99] F. James and M. Roos. Minuit: A System for Function Minimization and Analysis of the Parameter Errors and Correlations. Comput.Phys.Commun., 10:343-367, 1975.

[100] P. Adamson et al. Measurement of the neutrino mass splitting and flavor mixing by MINOS. Phys.Rev.Lett., 106:181801, 2011.

[101] Rashid Mehdiyev. MINOS Run Timeline. http://wwwnumi.fnal.gov/minwork/timeline/TimeLine.html, 2012.

[102] Andy Blake. Far detector pot integration for minos physics analyses. MINOS-doc-6806, 2012. 
[103] P. Adamson et al. Measurements of atmospheric neutrinos and antineutrinos in the MINOS Far Detector. Phys.Rev., D86:052007, 2012.

[104] G.L. Fogli, E. Lisi, A. Marrone, and A. Palazzo. Global analysis of threeflavor neutrino masses and mixings. Prog.Part.Nucl.Phys., 57:742-795, 2006.

[105] Laske G. Bassin, C. and G. Masters. The Current Limits of Resolution for Surface Wave Tomography in North America. EOS Trans AGU, F897:81, 2000.

[106] Luke Corwin. Mass Hierarchy Systematics: First Look at Earth Model Uncertainties. MINOS-doc-8955, 2012.

[107] J. K. et al Ahn. Observation of reactor electron antineutrinos disappearance in the reno experiment. Phys. Rev. Lett., 108:191802, May 2012.

[108] Y. Abe et al. Reactor electron antineutrino disappearance in the Double Chooz experiment. Phys.Rev., D86:052008, 2012.

[109] D.S. Ayres et al. The NOvA Technical Design Report. 2007.

[110] G. Tzanankos et al. MINOS+: a Proposal to FNAL to run MINOS with the medium energy NuMI beam. 2011.

[111] P. Adamson, J.A.B. Coelho, G.S. Davies, J.J. Evans, P. Guzowski, et al. CHerenkov detectors In mine PitS (CHIPS) Letter of Intent to FNAL. 2013.

[112] John Urish. Neutrino Beam Simulation using PAW with Weighted Monte Carlo. MINOS-doc-109, 1995.

[113] James Imber. Super-Kamiokande and T2K Joint Fit Studies for Neutrino Oscillation Parameters. DPF, 2013. 
[114] L. Camilleri. MicroBooNE. Nucl.Phys.Proc.Suppl., 237-238:181-183, 2013.

[115] P.A.N. Machado, H. Nunokawa, and R. Zukanovich Funchal. Testing for Large Extra Dimensions with Neutrino Oscillations. Phys.Rev., D84:013003, 2011.

[116] Joao A. B. Coelho. Three and four flavour oscillation results from MINOS. Fermilab Join Theory and Experimentalist Seminar, 2013. 